Ultra-High-Contrast Laser Acceleration of Relativistic Electrons in Solid Targets

D. P. Higginson

November 20, 2012 
This document was prepared as an account of work sponsored by an agency of the United States government. Neither the United States government nor Lawrence Livermore National Security, LLC, nor any of their employees makes any warranty, expressed or implied, or assumes any legal liability or responsibility for the accuracy, completeness, or usefulness of any information, apparatus, product, or process disclosed, or represents that its use would not infringe privately owned rights. Reference herein to any specific commercial product, process, or service by trade name, trademark, manufacturer, or otherwise does not necessarily constitute or imply its endorsement, recommendation, or favoring by the United States government or Lawrence Livermore National Security, LLC. The views and opinions of authors expressed herein do not necessarily state or reflect those of the United States government or Lawrence Livermore National Security, LLC, and shall not be used for advertising or product endorsement purposes.

This work performed under the auspices of the U.S. Department of Energy by Lawrence Livermore National Laboratory under Contract DE-AC52-07NA27344. 
UNIVERSITY OF CALIFORNIA, SAN DIEGO

\title{
Ultra-High-Contrast Laser Acceleration of Relativistic Electrons in Solid Targets
}

\author{
A dissertation submitted in partial satisfaction of the \\ requirements for the degree \\ Doctor of Philosophy \\ in \\ Engineering Sciences (Engineering Physics) \\ by \\ Drew Pitney Higginson
}

Committee in charge:

Professor Farhat Beg, Chair

Professor Sergei Krasheninnikov

Professor Kevin Quest

Professor Cliff Surko

Professor George Tynan 

The dissertation of Drew Pitney Higginson is approved, and it is acceptable in quality and form for publication on microfilm and electronically:

\section{Chair}

University of California, San Diego

2013 


\section{DEDICATION}

To Adriane. She inspires me to reach towards the stars and has taught me as much as a Ph.D. 
EPIGRAPH

Through truth we may find meaning. Though, probably not.

-Anonymous

Flesh is our fusion, our stardust, our sun, our orbit, our kiss upon kiss upon kiss -Adriane M. Tillman 


\section{TABLE OF CONTENTS}

Signature Page ...................... . . iii

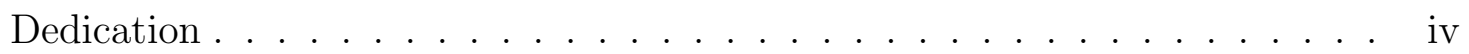

Epigraph .......................... . .

Table of Contents ......................... vi

List of Figures . . . . . . . . . . . . . . . . . ix

List of Tables ......................... . . . . . . . . . . . . . .

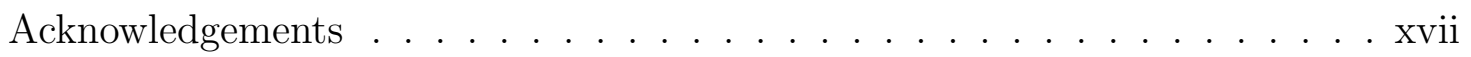

Vita and Publications .................... xix

Abstract of the Dissertation .................. . xxi

1 Background of Work . . . . . . . . . . . . . . . . . 1

1.1 Physics at Extreme Intensities with Short-Pulse Lasers . . . . . . . 1

1.2 Inertial Confinement Fusion . . . . . . . . . . . . . . . . 3

1.3 Fast Ignition . . . . . . . . . . . . . . . . . . . . . 6

1.4 Studies of Preplasma Effects on Electrons Acceleration . . . . . . . 11

1.5 Electron Source Determination and Cone-Wire Targets . . . . . . . 13

1.6 Outline of the dissertation . . . . . . . . . . . . . . 15

1.7 Role of the Author . . . . . . . . . . . . . . . . . . . 16

2 The Physics of Laser Plasma Interactions and Particle Transport . . . . 18

2.1 General Laser-Plasma Concepts . . . . . . . . . . . . . . . . 18

2.1.1 Shielding and the Debye Length . . . . . . . . . . . . . . 18

2.1.2 Plasma Frequency . . . . . . . . . . . . . . 20

2.1.3 Propagation of Laser Light Through a Plasma . . . . . . . . 21

2.2 Low-Intensity Interactions . . . . . . . . . . . . . . . . . 23

2.2.1 Field Ionization . . . . . . . . . . . . . . . . 23

2.2.2 Ablation ..................... 26

2.3 High-Intensity Interactions . . . . . . . . . . . . . . . 27

2.3.1 Laser Focusing and Defocusing Mechanisms . . . . . . . . . 27

2.3.2 Ponderomotive Force and Hole Boring . . . . . . . . . . . . 30

2.3.3 $\mathbf{j} \times \mathbf{B}$ Acceleration Mechanism . . . . . . . . . . . . . . 31

2.4 Electron Transport Physics . . . . . . . . . . . . . . . . . . 36

2.4.1 Collisional Effects and Plasma Resistivity . . . . . . . . . . 36

2.4 .2 Alfvén Current Limit . . . . . . . . . . . . . . . . . . 39 
2.4.3 Return Current Response Time . . . . . . . . . . . . . . . 40

2.4.4 Current Neutrality Conditions . . . . . . . . . . . . . . 42

2.4.5 Collisional Effects on Fast Particles . . . . . . . . . . . . 43

3 Laser Systems and Diagnostics _. . . . . . . . . . . . . . . 46

3.1 Laser System Descriptions . . . . . . . . . . . . . . . . . 46

3.1 .1 Laser Power . . . . . . . . . . . . . . . . . . . 47

3.1 .2 Laser Focus . . . . . . . . . . . . . . . . . . . . . 47

3.1 .3 Prepulse . . . . . . . . . . . . . . . . . . . 52

3.2 Overview of $\mathrm{K} \alpha$ Radiation . . . . . . . . . . . . . . . . . . 54

3.3 Crystal Spectrometer for K $\alpha$ Measurement . . . . . . . . . . . . 55

3.3.1 Bragg Reflection and Spectrometer Information . . . . . . . 55

3.3.2 Spectrometer Calibration with SHCCD . . . . . . . . . 58

$3.4 \mathrm{~K} \alpha$ Spherical Imager . . . . . . . . . . . . . . . . . . . . . 59

4 Particle-In-Cell Simulations . . . . . . . . . . . . . . . . . . 63

4.1 The direct-implicit PIC Method . . . . . . . . . . . . . . 63

4.2 Collisions and Resistivity . . . . . . . . . . . . . . 65

4.3 Benchmarking . . . . . . . . . . . . . 66

4.3.1 Benchmarking of Stopping Power . . . . . . . . . . . 67

4.3.2 Benchmarking of Scattering . . . . . . . . . . . 68

4.3.3 Benchmarking of $\mathrm{K}_{\alpha}$ x-ray Cross Sections . . . . . . . . 71

4.4 Equation-of-State Models in LSP . . . . . . . . . . . . . . 74

4.5 Proton Layer Benchmarking . . . . . . . . . . . . . . 75

5 Cone-Wire Experiments . . . . . . . . . . . . . . . . . 77

5.1 Experimental Setup . . . . . . . . . . . . . . . . . . 77

5.2 Coupling Efficiency . . . . . . . . . . . . . . . . . . . . 80

$5.3 \mathrm{~K}_{\alpha}$ Falloff Lengths . . . . . . . . . . . . . . . . . . . . 82

6 Cone-Wires as Diagnostics for Hot Electrons . . . . . . . . . . . 88

6.1 Sensitivity Range to Electron Energies . . . . . . . . . . . . . 88

6.2 Relationship between $\mathrm{K}_{\alpha}$ Emission and Total Electron Energy . . . 90

6.3 Electrostatic Charging . . . . . . . . . . . . . . . . . . . . . 92

6.3 .1 Wire charging . . . . . . . . . . . . . . 92

6.3 .2 Energy Fraction in Wire . . . . . . . . . . . . . . . . . 93

6.3 .3 Capacitance . . . . . . . . . . . . . . . . . . . . . . . 94

6.3 .4 Expected Values . . . . . . . . . . . . . . . . . . 95

6.4 Resistive Stopping . . . . . . . . . . . . . . . . 95

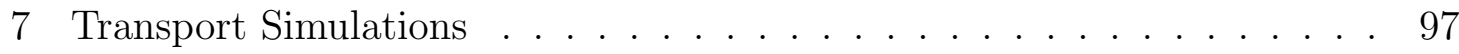

7.1 Simulation Setup . . . . . . . . . . . . . . . . . 97

7.2 Understanding the Transport of Electrons within the wire . . . . . 99

7.2.1 Initial Time: Electrons Initial Injection . . . . . . . . . . . 99 
7.2.2 Middle Time: Electrons Surf Along the Wire . . . . . . . . . 100

7.2.3 Final Time: Electrons Pushed into Wire . . . . . . . . . . . 103

7.3 Partition of Energy in the Simulation . . . . . . . . . . . . 105

$7.4 \mathrm{~K}_{\alpha}$ Production from Different Electron Energy Bins . . . . . . . . . 108

7.5 Temperature of background electrons and $\mathrm{K}_{\alpha}$ Shifting . . . . . . . 110

7.6 Single-Temperature Fits to the Data . . . . . . . . . . . . . 111

7.7 Assumptions in the Electron Distribution . . . . . . . . . . . 115

8 Laser-Plasma-Interaction Simulations . . . . . . . . . . . . . . . . . . 117

8.1 LPI Modeling of Short-Scale Preplasma . . . . . . . . . . . . 117

8.2 Absorption of Laser Energy . . . . . . . . . . . . . . . . . . . . 119

8.3 Accelerated Electron Distributions . . . . . . . . . . . . . . 121

8.3.1 Spatial and Temporal Profiles . . . . . . . . . . . . 122

8.3.2 Angular Distribution . . . . . . . . . . . . . . . . . 123

8.3.3 Energy Spectrum . . . . . . . . . . . . . . . . . . . . 125

8.4 Transport Simulations of LPI-measured Electron Distributions . . . 128

8.5 Preplasma Expansion Model . . . . . . . . . . . . . . . . . . . 131

8.6 LPI Modeling of Large-Scale Preplasma . . . . . . . . . . . . . . . 133

8.6.1 Coupling at Low-Contrast . . . . . . . . . . . . . . 134

8.6.2 Electron Spectra and Falloff Length at Low-Contrast . . . . 135

8.7 LPI Discussion . . . . . . . . . . . . . . . . . . . . . . 140

9 Summary . . . . . . . . . . . . . . . . . . . . . . . . . . . 142

A Transmission of a 3D cylinder . . . . . . . . . . . . . 147

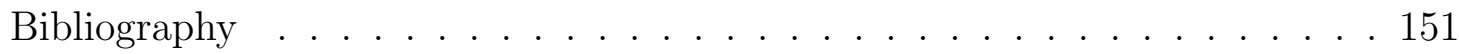




\section{LIST OF FIGURES}

Figure 1.1: Reactivity, $\langle\sigma v\rangle$, for various fusion reactions. Plotted using the fitting parameters from Bosch and Hale. ${ }^{[31]}$. . . . . . . . . . .

Figure 1.2: Density maps of the Isochoric Fast Ignition (FI) implosion (a) and the Central Hot Spot (CHS) implosion (b), and plots of the density $\rho$ and temperature $T$ of the implosion for the FI (c) and CHS $(\mathrm{d})$ cases. . . . . . . . . . . . . . . .

Figure 1.3: Gain curves for ignition for isochoric Fast Ignition (FI) and isobaric Central Hot Spot (CHS) ignition in solid and dotted lines, respectively. These are plotted using functions from ${ }^{[30]}$ with $\alpha=1.5 . \ldots \ldots \ldots \ldots \ldots \ldots$

Figure 1.4: (a) FI implosion scheme using an external source to provide compression, shown as lasers in gray, and (b) a high-intensity short-pulse laser to produce relativistic electrons to provide the heating for ignition. . . . . . . . . . . . . . 99 9

Figure 2.1: Offset of the ion and electron densities used for the derivation of the plasma frequency. . . . . . . . . . . . . . 20

Figure 2.2: The mechanism of multi-photon ionization. . . . . . . . . . . . 24

Figure 2.3: Ionization due to modification of the coloumb potential of an atom by an external (laser) electric field. . . . . . . . . . 25

Figure 2.4: Ionization curves for $\mathrm{Al}, \mathrm{Cu}$ and $\mathrm{Au}$, from. ${ }^{[58]}$ The ionization intensity is calculated from equation (2.30). . . . . . . . . 26

Figure 2.5: Focusing and defocusing of the laser based on changes in the index of refraction, $\eta$. . . . . . . . . . . . . . . 28

Figure 2.6: Plot of differential logarithms of $Z, \frac{\partial \log (Z)}{\partial I}$, and $\gamma_{0}, \frac{\partial \log \left(\gamma_{0}\right)}{\partial I}$, for given intensities. . . . . . . . . . . . . . . . . . . . 29

Figure 2.7: Motion of electrons and forces in a relativstic laser field. . . . . 35

Figure 2.8: Electron being scattered off multiple ions resulting in a random walk like movement. . . . . . . . . . . . . . . . . . 37

Figure 2.9: Resistivity curves for $\mathrm{Al}, \mathrm{Cu}$ and $\mathrm{Au}$ determined using the LMD resistivity model. . . . . . . . . . . . . . . 38

Figure 2.10: Plot of timescales of interest in return current generation. The laser frequency, $\omega_{\text {Laser }}$, is shown for a $1 \mu \mathrm{m}$ laser. The plasma frequency $\omega_{p e}$ is plotted. Return current decay time is plotted for two cases 1 ) for high value of resistivity $10^{-5} \Omega \mathrm{m}$ and 2 ) for metals at room temperature $10^{-8} \Omega \mathrm{m}$. . . . . . . . . . . 42

Figure 2.11: Energy loss, $\frac{d E}{d x}$, of electrons through solid density materials. . . 44

Figure 2.12: Average square deflection and Isotropic Scattering Distance for electrons in different materials at solid densities . . . . . . . . . 
Figure 3.1: Illustration of the CPA amplification technique. The short unamplified pulse, A, enters a stretcher (1) where the pulse is chirped by wavelength to a longer pulse length, B. This pulse enters the amplification train (2) and gains energy. This chirped, amplified pulse, C, then enters a compressor (3), which produces the amplified, high-power, short laser pulse, D. . . . . . . . .

Figure 3.2: Images of the two laser focal spots taken with high resolution cameras. The colorscale is linear with the maximum value normalized to the peak of the beams. . . . . . . . . .

Figure 3.3: Plots of the diffraction limited spots (an Airy function) and the experimentally observed Trident setup. . . . . . . . . . 50

Figure 3.4: Lineouts of the Trident and Titan Intensity profiles in the vertical and horizontal directions in the solid lines. These are compared to a triple gaussian that was used in simulations. . . . . .

Figure 3.5: Intensity Distributions of the Titan and Trident lasers for the minimum and maximum powers as listed in Table 3.1. Shown with a dotted line is the triple gaussian fit to the maximum intenisty. . . . . . . . . . . . . . . 52

Figure 3.6: Prepulse levels of the Trident (left) and Titan (right) Laser systems.The Trident level of prepulse, plotted on a log-scale) replotted from Ref. [18, is much lower than on Titan, plotted on a linear scale. . . . . . . . . . . . . . . .

Figure 3.7: Illustration of the generation of $\mathrm{K}_{\alpha}$ radiation. Ionizing radiation (e.g. a fast electron) ionizes a k-shell electron, which causes the $\mathrm{k}$-shell vacancy. When an electron from the L-shell drops to fill this vacancy it releases a photon with an energy equivalent to the difference in energy of the two states. This is called a $K_{\alpha}$ photon. . . . . . . . . . . . . . . 54

Figure 3.8: Cross-section for k-shell ionization in copper due to fast electrons. From equations in Refs. ${ }^{[70,71]}$. . . . . . . . . . . . 55

Figure 3.9: Illustration of Bragg reflection in a crystal. Incoming light rays $A$ and $B$ are reflected if they stay in phase. They rays will stay in phase if they traverse the same distance plus an integer, $n$, number of wavelenghts $\lambda$. If this condition is not met the light wave will not be in phase and thus not propagate. . . . . . . 56

Figure 3.10: Dimensions of the HOPG spectrometer. The shaded green line on top corresponds to a portion of the spectrometer use to diagnose $\mathrm{Ag} \mathrm{K}_{\alpha}$ radiation, which was not used in this work. The bottom portion in shaded gray corresponds to the $\mathrm{Cu} \mathrm{K}_{\alpha}$ that was used. Image courtesy of K. Akli . . . . . . . . . . . . 56 
Figure 3.11: (Left) Raw pseudo-color image of an imaging plate after exposure within the HOPG spectrometer. (Right) Transversely averaged lineout of the HOPG imaging plate, before and after subtraction of the continuum background radiation. . . . . . . 57

Figure 3.12: Chamber diagram of the cross-calibration setup for the SHCCD, not to scale. . . . . . . . . . . . . . . . . . . 58

Figure 3.13: (left) Raw image from SHCCD. The black pixels have energies consistent with $\mathrm{K} \alpha$ photons, while white pixels contain less energy and red pixels contain more energy than consistent with $\mathrm{K} \alpha$. (right) Spectra of energies contained on the SHCCD before the single event algorithm (to remove double hits) in blue and after in red. . . . . . . . . . . . . . . . . .

Figure 3.14: Calibration of the HOPG spectrometer against the SHCCD spectrometer. The dark orange dots are the experimental values with error bars. The dotted black line shows the calibration value. The shaded gray region corresponds to an error bar of $12 \%$, which is the value found from summation of the expected error bars from the calibration. The shaded orange region corresponds to an error of $27 \%$, which corresponds to the error expected from the deviation of these points from the average. .

Figure 3.15: Shifting of the $\mathrm{K}_{\alpha}$ image as a function of temperature. Image courtesy of F. Pérez. . . . . . . . . . . . . . . .

Figure 3.16: Ratio of integrated K $\alpha$ signal from the HOPG spectrometer versus the integrated signal from the $\mathrm{K} \alpha$ imager. No dependence on $\mathrm{K} \alpha$ signal level is observed and the ratios are fairly consistent, which is in agreement with the assumption that no shifting was observed. . . . . . . . . . . . . . . . .

Figure 4.1: Cartoon of a PIC simulation with macro-particles existing continuously in space and the fields calculated on a spatial grid. . . 63

Figure 4.2: Benchmarking results of the stopping power of electrons in $\mathrm{Cu} . \quad 68$

Figure 4.3: Setup of the LSP scattering benchmarking simulation. . . . . . 70

Figure 4.4: Distribution of angular momentum of $18 \mathrm{MeV}$ electrons after passing through a thin foil. The bars show a histogram and the line is a gaussian fit to the data. . . . . . . . . . . . . 70

Figure 4.5: Benchmarking of the electron angular scattering in LSP. . . . . 71

Figure 4.6: Plot of the Hombourger and Kolbenstvedt cross sections vs the experimental data. The data was taken from Ref. [82] and is named according the naming convention within the article. . . 72

Figure 4.7: $\mathrm{K}_{\alpha}$ generation per energy loss. . . . . . . . . . . . . . . 73

Figure 4.8: Conversion efficiency into $K_{\alpha}$ photons (linear scale). . . . . . . 73 
Figure 4.9: Equation-of-state in Copper. (Left) Heat capacity $C_{v}$ and (right) average ionization state Zbar as a function of electron temperature. . . . . . . . . . . . . . . 74

Figure 4.10: Comparison of cell size for proton acceleration from a thin foil. (Top) Cell size of $1 \mu \mathrm{m}$ with a conversion efficiency is $0.36 \%$. (Bottom) Cell size of $0.1 \mu \mathrm{m}$ with a conversion efficiency is $0.31 \% .76$

Figure 5.1: Dimensions of the cone-wire targets used in our experiments to diagnose the electron spectra of high-intensity lasers. The laser comes from the left in this diagram and is incident on the inner cone tip. . . . . . . . . . . . . . . . . 77

Figure 5.2: Photographs of the cone-wires. (left) Side on view of the wire and cone connections and (right) interior cone tip. . . . . . . 78

Figure 5.3: Diagram of the experimental setup for laser experiments using cone-wire targets. The setup was similar on both the Trident and Titan laser systems. The laser was used at normal incidence to the cone tip. The HOPG spectrometer and $\mathrm{K}_{\alpha}$ imager were the main diagnostics. . . . . . . . . . . . . . . . . . . . 79

Figure 5.4: K $\alpha$ coupling efficiency on the Trident and Titan laser systems. . 81 Figure 5.5: K $\alpha$ coupling efficiency on the Trident and Titan laser systems versus prepulse level. The Trident high contrast (purple triangles) and Titan Intrinsic Low Constrast (gray diamond) are from this work. The Titan injected Prepulse (red triangles) is also from the Titan experiment, but has been previously been published $^{[41]}$ and thus is not discussed further in this text. . . . 82

Figure 5.6: Raw images of the $\mathrm{K} \alpha$ radiation coming from the $\mathrm{Cu}$ wires. The colormaps are normalized to the peak signal. . . . . . . . . 83

Figure 5.7: Lineouts of the $\mathrm{K}_{\alpha}$ radiation coming from the $\mathrm{Cu}$ wires from (top) the Trident laser at 75 and (bottom) the Titan laser at 161 J. . . . . . . . . . . . . . . . . . 84

Figure 5.8: $\mathrm{K} \alpha$ falloffs on the high-contrast Trident (red triangles) and lowcontrast Titan (gray diamonds) laser systems. . . . . . . . . . . 86

Figure 6.1: Energy percentage remaining in mono-energetic electrons after passing through a $10 \mu \mathrm{m}$ Au block. . . . . . . . . . . 88

Figure 6.2: $\mathrm{Cu} \mathrm{K} \mathrm{K}_{\alpha}$ emission versus distance from mono-energetic electrons in an infinite $\mathrm{Cu}$ block. . . . . . . . . . . . . . . . . 89

Figure 6.3: Cross-section for k-shell ionization and stopping power for electrons in solid density $\mathrm{Cu}$. . . . . . . . . . . . . . . . 90

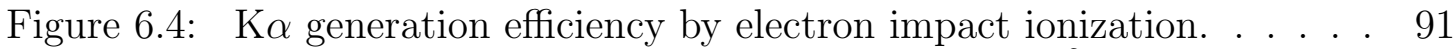

Figure 6.5: Energy fraction trapped in wire for a given $\frac{E_{e} q_{e}^{2}}{C T^{2}}$. . . . . . . . . 94

Figure 7.1: Setup of the transport simuations in LSP. . . . . . . . . . . . . 98 
Figure 7.2: Injected electron density plots during the first snap-shot of electron transport simualation at 2.5 ps. . . . . . . . . . . 100

Figure 7.3: Injected electron density plots during the second snap-shot of electron transport simualation at 5.6 ps. . . . . . . . . . . . . . 101

Figure 7.4: Radial lineouts of magnetic and electric forces (left). Measured and inferred magnetic fields (right) during the second snap-shot at $5.6 \mathrm{ps}$ taken near the surfing electrons at $1000 \mu \mathrm{m}$. . . . . . . 102

Figure 7.5: Cartoon of the generation of the current thorough charge imbalance. In (a) fast electrons leave the wire creating an electric field due to the charge difference. This draws a current (b) through the electric field, and this propagates through the wire (c). When the leading edge of the current reaches the end of the wire (d) it can no longer propagate, and creates a backward flowing current. This process will continue until the wire equilibriates to a constant potential. . . . . . . . . . . . . 103

Figure 7.6: Injected electron density plots during the third snap-shot of electron transport simualation at 7.1 ps. . . . . . . . . . . . . 104

Figure 7.7: Radial lineouts of magnetic and electric forces (left). Measured and inferred magnetic fields (right) during the third snap-shot at $7.1 \mathrm{ps}$ taken at $1400 \mathrm{\mu m}$ where electrons are being pushed into the wire. . . . . . . . . . . . . . . 105

Figure 7.8: Simulation of the $\mathrm{K}_{\alpha}$ emission profile using an injected electron distribution that reproduces the many features of the cone-wire experimental data. . . . . . . . . . . . . . . . 105

Figure 7.9: Partition of energy in the simulation as a function of time for single temperatures of 0.75 (left) and 2.0 (right) $\mathrm{MeV}$ slope temperatures. . . . . . . . . . . . . . . 106

Figure 7.10: Conversion efficiency into $\mathrm{K}_{\alpha}$ photon energy as a function of

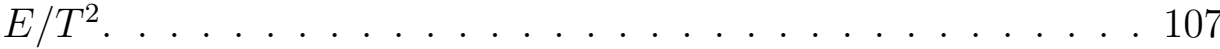

Figure 7.11: Profile of $\mathrm{K}_{\alpha}$ emission with and without a proton layer. . . . . . 108

Figure 7.12: Production of $\mathrm{K}_{\alpha}$ binned for the injected energies. . . . . . . . . 109

Figure 7.13: Cumulative $\mathrm{K}_{\alpha}$ production for given bins of injected electron energies, which show that $95 \%$ of the $\mathrm{K}_{\alpha}$ energy is produced by 0.5 to $10 \mathrm{MeV}$ electrons. . . . . . . . . . . . . . . . . . 110

Figure 7.14: Background electron temperature along the wire at different depths as a function of time. Here is the wire is initialized at $10 \mathrm{eV} \ldots \ldots \ldots \ldots \ldots \ldots \ldots$

Figure 7.15: Falloff distance vs temperature of a single exponetial with a $\mathrm{K} \alpha$ energy interpolated to $7.09 \mathrm{~mJ}$ to match of the Trident laser at $5 \times 10^{19} \mathrm{~W} / \mathrm{cm}^{2}$. The experimental falloff length for this data point is shown as a dotted line. . . . . . . . . . . . . . . 112 
Figure 7.16: Simulation of the $\mathrm{K}_{\alpha}$ profile for different injected slope temperatures. Experimental data for the Trident laser at $5 \times 10^{19}$ $\mathrm{W} / \mathrm{cm}^{2}$ is plotted in gray. . . . . . . . . . . . . . . . 112

Figure 7.17: Experimental and simulated $\mathrm{K}_{\alpha}$ lineouts from from (top) the Trident laser at $5 \times 10^{19} \mathrm{~W} / \mathrm{cm}^{2}$ and (bottom) the Titan laser

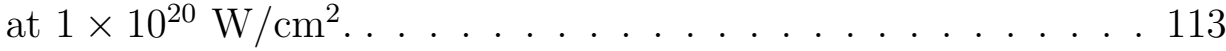

Figure 7.18: Slope temperatures of electrons inferred from single-temperature fits to the Trident and Titan data. . . . . . . . . . . . . . . . . 114

Figure 7.19: Fitting the $5 \times 10^{19} \mathrm{~W} / \mathrm{cm}^{2}$ shot Trident with a single exponential distribution while varying the angular distribution and injecting a hotter electron component with a $10 \mathrm{MeV}$ slope temperature. .................... 116

Figure 8.1: Electron number density in the initial setup of the LPI simua-

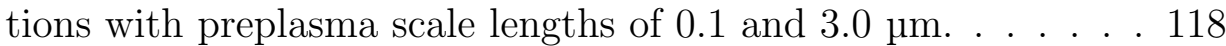

Figure 8.2: Absorption (circles) and coupling (squares) efficiency of electrons into the cone-tip. The closed markers represent the highcontrast data by using 0.1 to $3 \mu \mathrm{m}$ exponential profiles and the open markers represent the low-contrast case with much longer preplasma. . . . . . . . . . . . . . . . . . . 119

Figure 8.3: Number density and energy density of electrons in the LPI simulations of 0.1 and $3 \mu \mathrm{m}$ preplasma near the peak of the pulse at 1 ps. The dotted white represents the outline of the inner cone wall. . . . . . . . . . . . . . . . . . . . . 120

Figure 8.4: Time and spatial profiles of electrons going through the cone tip for the 0.1 and $3.0 \mu \mathrm{m}$ cases. Plots on the bottom show the laser distribution. The dotted lines in the spatial plots show the width of the wire.

Figure 8.5: Average angles of the electrons in the 0.1 and $3.0 \mu \mathrm{m}$ preplasma cases. . . . . . . . . . . . . . . . . . . . . . 124

Figure 8.6: Quasi-static, smoothed magnetic fields near the peak of the laser pulse at 1 ps. . . . . . . . . . . . . . . . . . . . . . . . . . . . . 124

Figure 8.7: Energy spectrum of the electrons passing through the cone-tip for the 0.1 and $3 \mu \mathrm{m}$ cases, shown for two different energy windows. 126

Figure 8.8: Profile of $\mathrm{K}_{\alpha}$ emission from the wire using the injected electron sources from the 0.1, 1 and $3 \mu \mathrm{m}$ preplasma LPI simulations and the experimental data for the $5 \times 10^{19} \mathrm{~W} / \mathrm{cm}^{2}$ case. . . . . 128

Figure 8.9: Comparison of the electron spectrum inferred from LPI modeling and the spectrum inferred from single temperature injections for the $5 \times 10^{19} \mathrm{~W} / \mathrm{cm}^{2}$ Trident case. . . . . . . . . . . . . 129

Figure 8.10: Profile of $\mathrm{K}_{\alpha}$ emission from the wire using the injected electron sources from the $0.1,1.8$ and $3 \mu \mathrm{m}$ preplasma LPI simulations and the experimental data for the $2 \times 10^{19} \mathrm{~W} / \mathrm{cm}^{2}$ case. . . . . 130 
Figure 8.11: Falloff distance of $\mathrm{K}_{\alpha}$ emission from the simulations at varying preplasma scale lengths plotted with the experimental data. . . 130

Figure 8.12: Preplasma density map expected on the Titan laser with $17 \mathrm{~mJ}$ of prepulse energy in 2 ns. . . . . . . . . . . . . . . . . 133

Figure 8.13: Profiles of the laser intensity, electron number density and electron energy density of the LPI simulations representing lowcontrast Titan interaction near the peak of the pulse. . . . . . . 134

Figure 8.14: Energy spectrum of electrons in the low-contrast (LC) case compared to the high-contrast (HC) cases. All of the $\mathrm{HC}$ spectra are taken at the cone-tip, while the LC data is take both at the tip and at 40um from the tip. . . . . . . . . . . . . . 136

Figure 8.15: Angular divergence distributions of the electrons at different energies taken near the source at $40 \mu \mathrm{m}$ from the inner cone-tip. 137

Figure 8.16: Profile of $\mathrm{K}_{\alpha}$ emission from the wire using the injected electron sources low-contrast simulations LPI simulation at $5 \times 10^{19}$ $\mathrm{W} / \mathrm{cm}^{2}$ and the experimental data from the $3 \times 10^{19} \mathrm{~W} / \mathrm{cm}^{2}$ case. 138

Figure 8.17: Simulated falloff distances as a function of preplasma scale length.139

Figure A.1: Sketch of a circle being viewed by observer at $x=\infty$. $L$ is the distance that the observer must view to see the point at $(\mathrm{x}, \mathrm{y})$ and $L_{0}$ is the length parallel to the x-axis from the outer edge of the cricle to the y-axis. . . . . . . . . . . . . . . . . . 147

Figure A.2: Sketch of a cylinder where $\gamma$ is the view angle, $L$ is the length to observer without the view angle and $L_{V}$ is the actually length to observer including the view angle. . . . . . . . . . . . . . 149

Figure A.3: The transmission, $T(R=20 \mu \mathrm{m}, \lambda=22 \mu \mathrm{m}, \gamma)$, and opacity $(\equiv 1 / T)$ plotted against the view angle of the wire, $\gamma . \ldots 149$ 


\section{LIST OF TABLES}

Table 3.1: Range of laser characteristics used in our experiments on the Trident and Titan laser systems. Also shown is the expected peak $\mathrm{T}_{\text {pond }}$ from the ponderomotive potential in (2.65), where

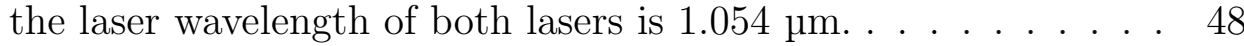

Table 3.2: Comparison of the Trident and Titan focal spots versus diffraction limited beams. . . . . . . . . . . . . . . . . . . 49

Table 3.3: Comparison of the Trident and Titan focal spots with respect to the energy contained in a region of the beam. . . . . . . . . 50

Table 3.4: Triple gaussian function that fits the Trident focal spot and is also quite similar to the average of Titan. The peak intensity is the intensity when fit to the maximum energy Trident shot. The peak fraction is the normalized fraction of the peak. The FWHM is the full-width-at-half-maximum of the gaussian and the energy fraction is the fraction of energy the particular gaussian function contains with respect to the total. . . . . . . . . . . . 51

Table 8.1: Parameters of triple exponential fits to the electron spectra. Temperatures $T$ and fraction of energy $F$ contained for each exponential. . . . . . . . . . . . . 127

Table A.1: Transmission, $T(R=20 \mu \mathrm{m}, \lambda=22 \mu \mathrm{m}, \gamma)$, and opacity $(\equiv 1 / T)$ for view angles, $\gamma \ldots \ldots \ldots \ldots \ldots \ldots$ 


\section{ACKNOWLEDGEMENTS}

First of all, I thank my advisor Prof. Farhat Beg for responding to my email inquiry about doing an undergraduate summer internship in San Diego those many years ago. Without that, none of this would have been possible! I appreciate both the guidance and the freedom that he has given me. I am fortunate that he is an advisor who spares no expense to give his students opportunities to excel by flying them around the world to pursue interesting physics and experiments where ever they are to be found. I also am grateful to Richard Stephens for mentoring me in my entrance into Plasma Physics as a intern at General Atomics; his extensive knowledge was and continues to be useful.

I thank my advisor Prav Patel for his technical guidance and for giving me direction when I got lost looking into details. His sharp mind helped me to pick approaches to understanding the data and presenting an effective story. My advisor Jim McNaney taught me much about doing good experiments and working in a fast-paced environment.

A great thanks is given to Tony Link. His expertise with the LSP simulations and physics guidance were extremely useful to my work, and it may not have been possible without him! Hiroshi Sawada was a great resource for me and we shared many intense and useful discussions on cone-wires. Scott Wilks helped me to get started with simulations; his upbeat attitude and physical insight were uplifting when the path was rough.

I am extremely grateful to the team at LLNL, UCSD and other institutions for teaching me much and working hard through long hours on experiment. Thanks goes to Kramer Akli, Teresa Bartal, Sophie Baton, Claudio Bellei, Michelle Burke, Sugreev Chawla, Cliff Chen, Jim Cobble, Rich Combs, Jack Davis, Bob Fedosejevs, Kirk Flippo, Rick Freeman, Johan Frenje, Hal Friesen, Sandrine Gaillard, Vladimir Glebov, Daniel Hey, Charlie Jarrott, Elijah Kemp, Andy Krygier, Kate Lancaster, Tammy Ma, Andy Mackinnon, Alessio Morace, Hirotaka Nakamura, Nobuhiko Nakanii, Peter Norreys, Dustin Offerman, Chris Orban, Frédéric Pérez, George Petrov, Yuan Ping, Bin Qiao, João Santos, Doug Schumacher, Anna Sorokovikova,

Christian Stoeckl, Damian Swift, Jack Topper, Linn Van Woerkom, Mingsheng 
Wei, Brad Westover, and Toshi Yabuuchi. I also thank the Titan, Trident and Luli laser facility teams.

I am greatly appreciative of the opportunity and financial support that the Lawrence Scholarship gave me to work at LLNL. Working here has taught me the expectations of doing cutting edge science and has broadened my horizons through conversions with great minds in the field. Group meetings with Bruce Cohen, Laurent Divol, Andreas Kemp, Mike Key, Dave Larson, Dave Strozzi, and Max Tabak have taught me the importance of aggressively defending strong research, while quickly seeing through broad-statements and fuzzy science.

Thank you to my mother and father, and the rest of my family, for their constant encouragement and for taking pride in my accomplishments even when they didn't really know what I was doing! Thank you to the Tillman family for supporting me as one of there own and for graciously inviting me into their homes in both San Diego and Oxford. I thank all of my friends in San Diego, San Francisco, Oakland and across the world for helping to make my graduate years exciting outside of work as well. Finally, I thank Adriane for fighting along side me in the tough times, pushing me to do my best at all times, and celebrating with me in the good times.

This work performed under the auspices of the U.S. Department of Energy by Lawrence Livermore National Laboratory under Contract DE-AC52-07NA27344. 
VITA

\section{PUBLICATIONS}

\section{First and Second Author}

D. P. Higginson, et al., Ultra-High-Contrast Laser Rise-Time Effects in the Acceleration of Relativistic Electrons in Solid Targets, in preparation for submission.

D. P. Higginson, et al., Production of Neutrons up to $18 \mathrm{MeV}$ in High-Intensity, Short-Pulse Laser Matter Interactions, Physics of Plasmas 18, 100703 (2011).

D. P. Higginson, et al., Laser Generated Neutron Source for Neutron Resonance Spectroscopy, Physics of Plasmas 17, 100701 (2010).

G. Petrov, D. P. Higginson, et al., Generation of High-Energy (>15 MeV) Neutrons using Short Pulse High Intensity Lasers, Physics of Plasmas 19, 093106 (2012).

H. Sawada, D. P. Higginson, et al., Characterizing the Energy Distribution of Laser-Generated Relativistic Electrons in Cone-Wire Targets, Physics of Plasmas 19, 103108 (2012).

\section{2}

T. Ma, et al., Hot Electron Temperature and Coupling Efficiency Scaling with Prepulse for Cone-Guided Fast Ignition, Physical Review Letters 108, 115004 (2012).

B. S. Paradkar, et al., Emission of Energetic Protons from Relativistic Intensity Laser Interaction with a Cone-Wire Target, Physical Review E 86, 056405 (2012).

Y. Ping, et al., Dynamics of Relativistic Laser-Plasma Interaction on Solid Targets, Physical Review Letters 109, 145006 (2012). 
T. Bartal, et al., Focusing of Short-Pulse High-Intensity Laser-Accelerated Proton Beams, Nature Physics 7, 1 (2011).

F. Pérez, et al., Magnetically Guided Fast Electrons in Cylindrically Compressed Matter, Physical Review Letters 107, 065004 (2011).

B. Vauzour, et al., Laser-Driven Cylindrical Compression of Targets for Fast Electron Transport Study in Warm and Dense Plasmas, Physics of Plasmas 18, 043108 (2011).

L. Volpe, et al., Proton Radiography of Laser-Driven Imploding Target in Cylindrical Geometry, Physics of Plasmas 18, 012704 (2011).

\section{0}

K. U. Akli, et al., A Dual Channel x-ray Spectrometer for Fast Ignition Research, Journal of Instrumentation 5, P07008 (2010).

P. A. Norreys, et al., New Developments in Energy Transfer and Transport Studies in Relativistic Laser-Plasma Interactions, Plasma Physics and Controlled Fusion 52, 124046 (2010).

F. Perez, et al., Single-Shot Divergence Measurements of a Laser-Generated Relativistic Electron Beam, Physics of Plasmas 17, 113106 (2010).

B. Vauzour, et al., Experimental Study of Fast Electron Propagation in Compressed Matter, Nuclear Instruments and Methods in Physics Research A 653, 176 (2010).

\section{9}

C. R. D. Brown, et al.,, Radiation and Hot Electron Temperature Measurements of Short-Pulse Laser Driven Hohlraums, High Energy Density Physics 5, 212 (2009).

C. D. Chen, et al., Bremsstrahlung and k $\alpha$ Fluorescence Measurements for Inferring Conversion Efficiencies into Fast Ignition Relevant Hot Electrons, Physics of Plasmas 16, 082705 (2009).

F. Perez, et al., Fast-Electron Transport in Cylindrically Laser-Compressed Matter, Plasma Physics and Controlled Fusion 51, 124035 (2009). 


\title{
ABSTRACT OF THE DISSERTATION \\ Ultra-High-Contrast Laser Acceleration of Relativistic Electrons in Solid Targets
}

\author{
by \\ Drew Pitney Higginson \\ Doctor of Philosophy in Engineering Sciences (Engineering Physics) \\ University of California, San Diego, 2013 \\ Professor Farhat Beg, Chair
}

The cone-guided fast ignition approach to Inertial Confinement Fusion requires laser-accelerated relativistic electrons to deposit kilojoules of energy within an imploded fuel core to initiate fusion burn. One obstacle to coupling electron energy into the core is the ablation of material, known as preplasma, by laser energy proceeding nanoseconds prior to the main pulse. This causes the laser-absorption surface to be pushed back hundreds of microns from the initial target surface; thus increasing the distance that electrons must travel to reach the imploded core. Previous experiments have shown an order of magnitude decrease in coupling into surrogate targets when intentionally increasing the amount of preplasma. Additionally, for electrons to deposit energy within the core, they should have kinetic 
energies on the order of a few $\mathrm{MeV}$, as less energetic electrons will be stopped prior to the core and more energetic electrons will pass through the core without depositing much energy. Thus a quantitative understanding of the electron energy spectrum and how it responds to varied laser parameters is paramount for fast ignition.

For the first time, this dissertation quantitatively investigates the acceleration of electrons using an ultra-high-contrast laser. Ultra-high-contrast lasers reduce the laser energy that reaches the target prior to the main pulse; drastically reducing the amount of preplasma. Experiments were performed in a cone-wire geometry relevant to fast ignition. These experiments irradiated the inner-tip of a $\mathrm{Au}$ cone with the laser and observed electrons that passed through a $\mathrm{Cu}$ wire attached to the outer-tip of the cone. The total emission of $K_{\alpha}$ x-rays is used as a diagnostic to infer the electron energy coupled into the wire. Imaging the $\mathrm{x}$-ray emission allowed an effective path-length of electrons within the wire to be determined, which constrained the electron energy spectrum.

Experiments were carried out on the ultra-high-contrast Trident laser at Los Alamos National Laboratory and at the low-contrast Titan laser at Lawrence Livermore National Laboratory. The targets were irradiated using these $1.054 \mathrm{\mu m}$ wavelength lasers at intensities from $10^{19}$ to $10^{20} \mathrm{~W} / \mathrm{cm}^{2}$. The coupling of energy into the $\mathrm{Cu}$ wire was found to be $2.7 \mathrm{x}$ higher when the preplasma was reduced using high-contrast. Additionally, higher laser intensity elongated the effective path-length of electrons within the wire, indicating that their kinetic energy was higher.

To understand the physics behind laser-acceleration of electrons and to examine how this mechanism is affected by the presence of preplasma, simulations were performed to model the laser interaction. This simulations modeled the interaction using a 0.1 to $3 \mu \mathrm{m}$ exponential preplasma scale length for the high-contrast cases and hydronamically simulated longer scale preplasma $(\sim 25 \mu \mathrm{m})$ for the lowcontrast case. The simulations show that absorption of laser light increases from only $20 \%$ with a $0.1 \mu \mathrm{m}$ scale length to nearly $90 \%$ with a long low-contrast-type preplasma. However, as observed in experiments, a smaller fraction of this ab- 
sorbed energy is transported to the diagnostic wire, which is due to an increased distance that the electrons must travel to reach the wire and increase angular divergence of the electrons. The simulations show that increasing the preplasma scale length from 0.1 to $3 \mu \mathrm{m}$ increases the average energy by a factor of $2.5 \mathrm{x}$. This is consistent with an increased interaction length over which the electrons can gain energy from the laser.

The simulated electrons are compared with experimental data by injecting them into another simulation modeling the transport of electrons through the conewire target. This method quantitatively reproduced the experimentally measured the $\mathrm{K}_{\alpha} \mathrm{x}$-ray emission profiles in the high-contrast cases, which gives confidence in the simulations and the generated electron distributions.

By showing that the reduction of preplasma increases coupling into surrogate targets this work shows a significant advantage for the fast ignition scheme. Such work gives confidence to facilities that increasing the contrast of their laser systems will increase electron coupling. Additionally, detailed investigation of these high-contrast systems will aid researchers in understanding the effect that preplasma has on the acceleration of electrons. 


\section{Background of Work}

\subsection{Physics at Extreme Intensities with Short- Pulse Lasers}

In 1985, Donna Strickland and Gérard Mourou ${ }^{[1]}$ applied a technique called chirped-pulse amplification (CPA) to lasers. This method revolutionized laser physics by opening the door to extremely high-intensities $\left(>1 \mathrm{PW}^{[2]}\right)$ and extremely short pulse lengths $\left(\sim 30 \mathrm{fs}^{[3]}\right)$. The CPA method stretches out the pulse in time before amplification to allow a large amount of energy $\left(>1 \mathrm{~kJ}^{[4]}\right)$ to be gained. Then after amplification, the pulse is re-compressed to a short pulse length with extremely high intensity.

As an example of these high-intensity lasers, let us consider the Titan laser system at the Lawrence Livermore National Laboratory. This laser is capable of achieving peak intensities of $10^{20} \mathrm{~W} / \mathrm{cm}^{2}$. The laser energy is $150 \mathrm{~J}$ with a pulse duration of $0.7 \mathrm{ps}$, thus is has a power exceeding $200 \mathrm{TW}$. Thus the average power of the laser exceeds the average power consumption on Earth (16 TW in 2008 ${ }^{[5]}$ ); a piece of trivia often cited for tour groups. The laser intensity produced on Titan creates electric fields on the order of $10^{13} \mathrm{~V} / \mathrm{m}$, which are capable of ionizing atoms to high charge states by ripping the electrons from their shells. Ionization calculations (explained in detail in Section 2.2.1) show that such fields are capable of ionizing Gold to an ionization state above $40+$. Thus, these lasers create a plasma instantly, where ever they are incident.

An important feature of these lasers is the acceleration of electrons to relativistic energies. This acceleration is due not only to the strong electric fields of 
the laser, but also to the magnetic fields due to the $\mathbf{v} \times \mathbf{B}$ force, which becomes effective as the electrons approach the speed of light.

The lasers are capable of coupling $20-40 \%$ of the laser energy into electrons ${ }^{[6]}$ with kinetic energies above $1 \mathrm{MeV}$. At such energies the electrons can propagate large distances into solid density targets. For example, a $1 \mathrm{MeV}$ electron has a range over $1 \mathrm{~mm}$ in solid Al. This large range, along with the fast speed and short duration of the pulse, means that these electrons can isochorically heat materials to temperatures above $100,{ }^{[7]}$ or even $1000^{[8]} \mathrm{eV}$. Such heating at high-density creates matter in the warm dense matter (WDM) regime, which is interesting in the study of planetary and astrophysical research. ${ }^{[9]}$

When accelerated in a thin $(<100 \mu \mathrm{m})$ foil, the electrons can create strong static electric fields when escaping from the target into vacuum. The large charge imbalance can create an electric field up to $\mathrm{TV} / \mathrm{m}$; so strong that it ionizes material and accelerates the charged ions. ${ }^{[10-17]}$ Such a method has been used to accelerate protons to energies above $60 \mathrm{MeV} .{ }^{[18]}$ By modifying the target surface, the electric fields can also be used to focus the ion beam to $10 \mu \mathrm{m}$ diameter spots. ${ }^{[19]}$ The accelerated ions can potentially be used for medical oncology treatments or to produce high energy neutron sources. ${ }^{[20-22]}$

These high-intensity lasers can create extremely high electron currents. If we consider the Titan laser system, average electron energies are on the order of 1 $\mathrm{MeV}$, with $150 \mathrm{~J}$ of laser energy and a conversion efficiency of $20 \%$, and the pulse length about $700 \mathrm{fs}$ in duration. This leads to a peak current of over $10 \mathrm{MA}$. To get an idea of the magnitude of these currents, we can calculate the Alfvén current. ${ }^{[23]}$ This is the current at which a beam of electrons generates a strong enough magnetic field to deflect the beam onto its own axis. For a $1 \mathrm{MeV}$ electron beam the Alfvén current $^{1}$ is $33 \mathrm{kA}$. Thus the current generated by the Titan laser system is 300 times greater, and would not be able to propagate if not for a counter-propagating background current that allows for current quasi-neutrality. ${ }^{[24]}$

These high current and high energy-content relativistic electron beams were identified as a potential mechanism to ignite an inertial confinement fusion reac-

\footnotetext{
${ }^{1}$ Alfvén current is calculated at $I_{A}[A]=\frac{\mathcal{E}}{30 \mathrm{eV}}$, where $I_{A}$ is the Alfvén current and $\mathcal{E}$ is the energy of electrons. ${ }^{[23]}$
} 
tion. This idea is called Fast Ignition, ${ }^{[25]}$ but to explain it, we must first discuss the conventional method of central hot spot (CHS) inertial confinement fusion (ICF) and related subjects.

\subsection{Inertial Confinement Fusion}

Nuclear fission, the splitting of atoms, was first recognized by Meitner and Frisch $^{[26]}$ in 1939 as an explanation for the formation of barium isotopes upon the bombardment of uranium with neutrons. ${ }^{[27]}$ Less than 5 years later, in 1942, the first sustained nuclear fission reaction was demonstrated by Enrico Fermi at the University of Chicago housed within a large fabric tent on Squash court. ${ }^{[28]}$ On the other hand, Fusion, the joining of atoms, had been discovered even earlier in 1933 by the students of Lord Ernest Rutherford, who famously stated:

The energy produced by the breaking down of the atom is a very poor kind of thing. Anyone who expects a source of power from the transformation of these atoms is talking moonshine. Lord Ernest Rutherford, 1933 ${ }^{[29]}$

Rutherford was describing fusion induced by protons incident on lithium atoms, and perhaps he had some prescience. For now, nearly 80 years later not one fusion power plant has been created! Though fortunately, fusion research continues to be an active and exciting area of research. The fundamental difference between fusion and fission lies in the fact that energy creating fission can be induced with uncharged neutrons that easily penetrate the nucleus. Fusion, on the other hand, requires the joining of two charged nuclei, which therefore must be energetic enough to tunnel through the coulomb barrier. This requires heating the fuel to temperatures near $10 \mathrm{keV}$ (over 11 million degrees Kelvin).

$$
E_{\text {fusion }}=\frac{M_{i}}{\bar{m}_{i}} \times Q \times\langle\sigma v\rangle \times n_{i} \tau
$$

To calculate the energy produced by fusion reactions, we calculate the number of reactions that occur and multiply the energy that each reaction produces, $Q$. The equation (1.1) provides a relation for the energy produced per a given mass $M_{i}$ of fuel through simple zero-dimensional analysis. ${ }^{[30]}$ Here $m_{i}$ is a average 
mass per ion of the fuel, $Q$ is the energy released by the fusion reaction, $n_{i}$ is the cumulative density of the fuel, and $\tau$ is the amount of time that the fuel is confined and has the required temperature to produce fusion. The reactivity $\langle\sigma v\rangle$ measures the number of reactions that occur per unit density and per unit time. It is calculated by integrating the thermal velocity distribution $f_{M}(v)$ of the ions multiplied by the ion velocity $v$ and the cross-section of the reaction $\sigma(v)$; mathematically $\langle\sigma v\rangle=\int f_{M}(v) \sigma(v) v d v$. For reasons related to both the cross-section of the reaction and the thermal distribution of the ions, the reactivity is a strong function of temperature, as seen in Figure 1.1.

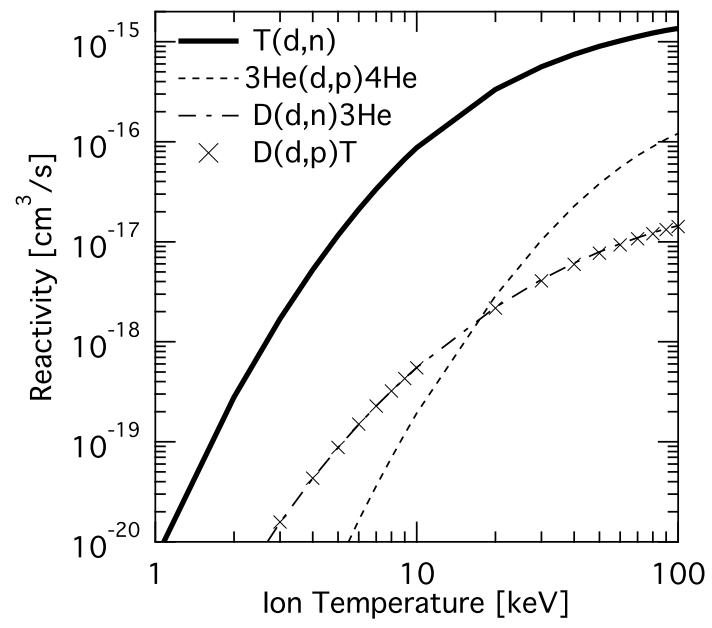

Figure 1.1: Reactivity, $\langle\sigma v\rangle$, for various fusion reactions. Plotted using the fitting parameters from Bosch and Hale. ${ }^{[31]}$

From equation (1.1), we know that a reaction with a high $Q$-value and high reactivity is desired for fusion energy production. Due to its high $Q$-value of $17.6 \mathrm{MeV}$ and high reactivity at relatively low temperatures (see Figure 1.1), the DT (deuterium-tritium) reaction is generally considered the first generation fuel for fusion energy. The energetic products of this reaction are a neutron and an $\alpha$-particle, which receive $3.5 \mathrm{MeV}$ and $14.1 \mathrm{MeV}$, respectively, when reaction temperatures are much less than the $Q$-value.

$$
{ }_{1}^{2} \mathrm{D}+{ }_{1}^{3} \mathrm{~T} \rightarrow \alpha(3.5 \mathrm{MeV})+\mathrm{n}(14.1 \mathrm{MeV})
$$


Once an appropriate fuel and reaction temperature (to determine $\langle\sigma v\rangle$ ) are chosen, the energy production is a function of only the density $n_{i}$ and the confinement time $\tau$. The multiple $n_{i} \tau$ is a useful value in determining the effectiveness of fusion production in a plasma. This value also is found in the Lawson criterion, ${ }^{[32]}$ which was originally developed for magnetic fusion and relates the energy created by fusion to the energy lost from the system (e.g. radiative losses, diffusive losses). Obviously, large amount of fusion energy are desired, which requires high values of $n_{i} \tau$.

The two major approaches to fusion are magnetic confinement fusion (MCF) and inertial confinement fusion (ICF), which use magnetic fields and the inertia of an imploding capsule to confine the fuel, respectively. While both methods must achieve high values of $n_{i} \tau$, the approach in MCF uses low density $\left(\sim 10^{14} \mathrm{~cm}^{-3}\right)$ and long confinement times of seconds to hours, while the ICF approach is orders of magnitude higher in density $\left(\sim 10^{25} \mathrm{~cm}^{-3}\right)$, but orders of magnitude lower in confinement times on the order of picoseconds. ${ }^{[30]}$

In this dissertation, we will focus only on ICF, where the exterior a small capsule is heated so that outer material is ablated, creating a rocket-like effect that creates a spherical implosion, compressing the DT fuel to 100's of times solid density. At peak compression the target achieves a mass density of $\rho=m_{i} n_{i}$ with radius $R$. The confinement time is given by the time it takes for information to travel from the center to the edges of the compressed fuel, since the information transfer occurs at the speed of sound, $c_{s}$, giving a confinement time of $\tau=R / c_{s}$. Thus in ICF we see a relationship between $n_{i} \tau$ and $\rho R$ as shown in equation (1.3). This value is related to the confinement time, so higher values of $\rho R$ will create more fusion energy. Additionally, a larger value of $\rho R$ will allow the $\alpha$-particles to deposit more energy along a given distance.

$$
\rho R=\frac{1}{m_{i} c_{s}} n_{i} \tau
$$

One might assume that in ICF the compressed fuel is heated to a uniform temperature through the compression of the capsule. However, the external heating of the fuel is too inefficient to produce a significant gain in energy through this 
method. Instead, only a small region within the fuel is heated externally and the $\alpha$-particles produced by fusion reactions in the hot spot propagate through the compressed fuel to heat the rest of it. This is known as Central Hot Spot ignition (CHS), as the region in the center of the fuel is called the hot spot.

This hot spot is relatively low density and is surrounded by denser fuel. In order to reach fusion temperatures the hot spot must get much hotter than the surrounding colder, dense fuel. As the capsule reaches stagnation, it is pressure equilibrium with the surrounding fuel because its sound speed is much faster than the stagnation time. Thus this method is known as isobaric ignition. In order to achieve a high amount of compression and heating, the capsule must be compressed incredibly uniformly. This requires extremely uniform target manufacturing and extremely symmetric target heating during the implosion. A method that aims to increase the gain from ICF as well as reduce this uniformity requirements is known as Fast Ignition.

\subsection{Fast Ignition}

The fast ignition (FI) concept was proposed by Tabak, et al. in 1994. ${ }^{[25]}$ This concept separates the implosion phase of the capsule from the heating phase of the fuel. As in CHS, the implosion is driven using nanosecond lasers. However, the imploded fuel is then heated by a burst of electrons generated by a laser pulse around 10 ps. Because the implosion does not need to create a compressed hot spot, the symmetry requirements for the implosion are greatly reduced.

Additionally the capsule is ignited isochorically instead of isobarically, as illustrated in Figure 1.2. In an isochoric ignition, like in FI, the mass of the target is contained in an imploded sphere, where as in CHS the majority of the mass is contained in a compressed shell at some radius from the center. Geometrically, this creates a higher $\rho R$, for a given density and mass, simply because more of the fuel is closer to the center. For instance, a factor of 4 increase in $\rho R$ in the FI case versus the CHS case is expected. ${ }^{[30]}$

In FI, the heating of the fuel must occur before the compressed fuel expands. 


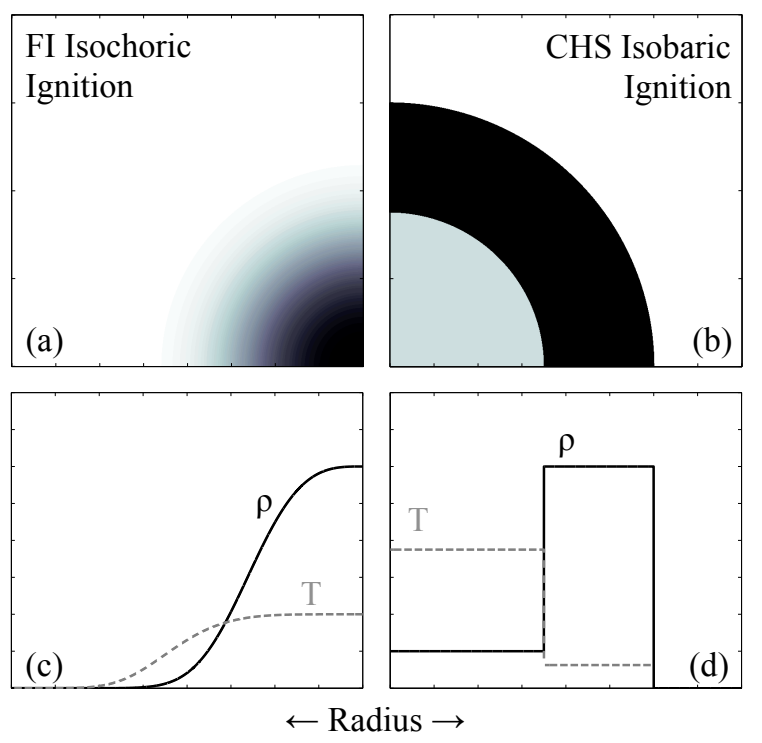

Figure 1.2: Density maps of the Isochoric Fast Ignition (FI) implosion (a) and the Central Hot Spot (CHS) implosion (b), and plots of the density $\rho$ and temperature $T$ of the implosion for the FI (c) and CHS (d) cases.

The time for this to happen is determined by calculating how long information, moving at the sound speed, takes to travel from the center of the compressed spot to its boundary. Ignition conditions achieving fusion gain using isochoric ignition are given by Atzeni and Meyer-ter-Vehh ${ }^{[30]}$ as $\rho R=0.5 \mathrm{~g} \mathrm{~cm}^{-2}$ and $T=12 \mathrm{keV}$, which requires an input energy of $E=\left(850 \mathrm{~g} \mathrm{~cm}^{-3} / \rho\right)^{2} \mathrm{~kJ}$. To attain the proper $\rho R$ for a density of $300 \mathrm{~g} / \mathrm{cm}^{3}$ the compressed spot must be around $20 \mu \mathrm{m}$. With the given temperature of $12 \mathrm{keV}$ the plasma will have a sound speed of about $10^{8}$ $\mathrm{cm} / \mathrm{s}$, which gives a hydrodynamic breakup time of about $15 \mathrm{ps}$. Also, this requires around $18 \mathrm{~kJ}$ of deposited electron energy. ${ }^{[33]}$

The gain calculated by Atzeni and Meyer-ter-Vehh ${ }^{[30]}$ for isochoric Fast Ignition and isobaric central hot spot ignition are given by (1.4) and (1.5), respectively. Here $G$ is the gain and $E_{\text {fuel }}$ is the total internal energy in the compressed fuel, which is related to the nanosecond driver energy. The isentrope parameter is $\alpha$, which is a measurement of the fuel entropy compared to cold fermi-degenerate 
conditions, and is usually from 1.5 to $4 .{ }^{[30]}$

$$
\begin{aligned}
G_{\mathrm{FI}} & =19.2 \times 10^{3}\left(\frac{E_{\text {fuel }}[M J]}{\alpha^{3}}\right)^{0.4} \\
G_{\mathrm{CHS}} & =5.6 \times 10^{3}\left(\frac{E_{\text {fuel }}[M J]}{\alpha^{3}}\right)^{0.3}
\end{aligned}
$$

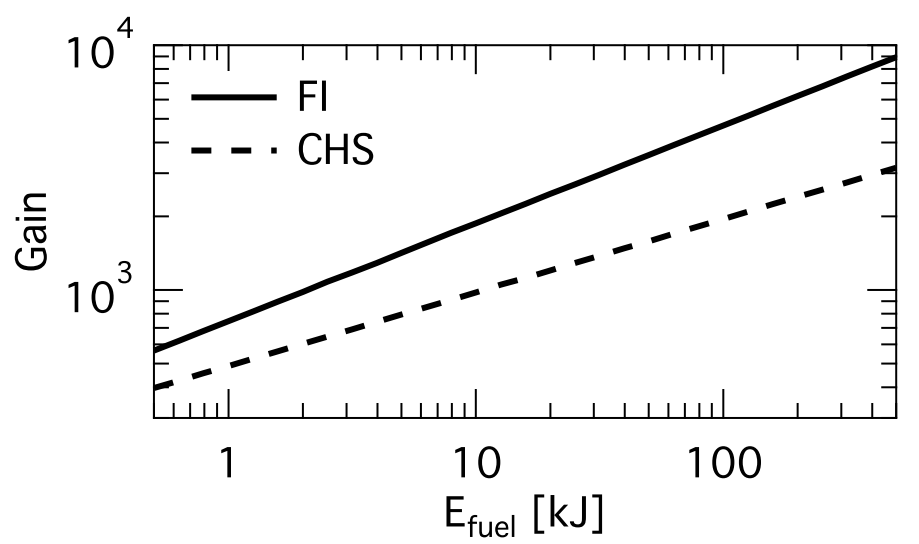

Figure 1.3: Gain curves for ignition for isochoric Fast Ignition (FI) and isobaric Central Hot Spot (CHS) ignition in solid and dotted lines, respectively. These are plotted using functions from ${ }^{[30]}$ with $\alpha=1.5$.

The gain curves for FI and CHS are plotted in Figure 1.3. These show that the gain for FI is about 2 to 3 times higher at fuel energies between 5 to $50 \mathrm{~kJ}$. Additionally, for a given gain the energy required is a factor of 7 to 10 times lower. This suggests that FI may be possible with lower nanosecond driver energies and potentially less capital investment when building fusion power plants.

While the advantages of FI are promising, a significant uncertainty is in how the picosecond laser will accelerate electrons and how well these electrons are able to deposit energy within the core.

During the capsule implosion, material will be ablated from the capsule and extend many millimeters from the compressed core. Since the compressed core is only around $20 \mu \mathrm{m}$, the solid angle of this core is very small if electrons are accelerated from millimeter distances. Thus a way must be found to allow the laser to get much closer to the core before it interacts with the plasma. The initial idea proposed by Tabak ${ }^{[25]}$ was to use another laser to bore a hole through 
this ablated plasma. However, this requires two lasers and makes the process more complicated. Presently, the most prevalent idea is to use a cone ${ }^{[34-36]}$ as shown in Figure 1.4. The cone keeps a pathway clear of ablated plasma during the implosion so that the short pulse laser can propagate unimpeded to a point near to the imploded core. This non-symmetric geometry make the implosion more complicated than a conventional spherically symmetric implosion. However, hydrodynamic simulations have shown that compression is still feasible in this conegeometry $^{[37]}$ and can create an imploded core of around $300 \mathrm{~g} / \mathrm{cm}^{3}$ at a $100 \mu \mathrm{m}$ stand-off distance from the outer cone-tip.
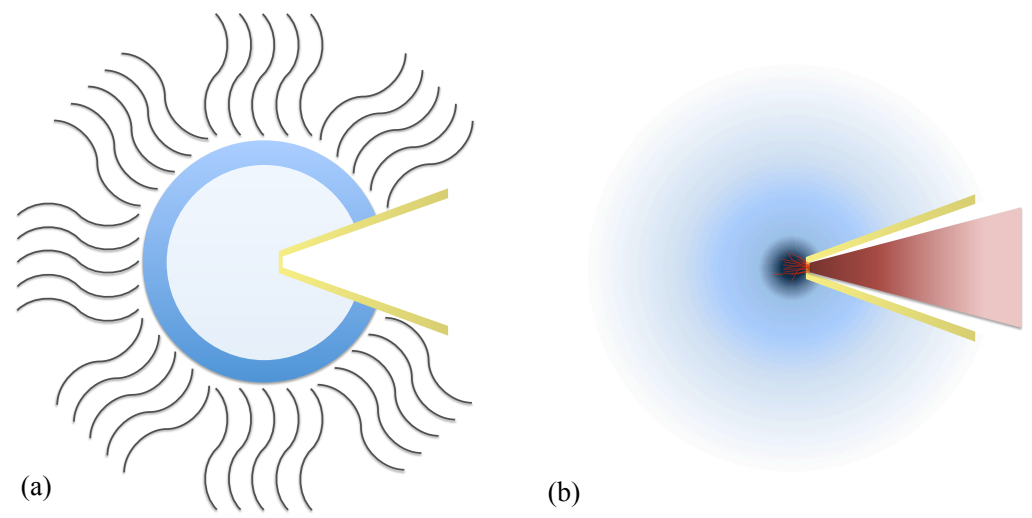

Figure 1.4: (a) FI implosion scheme using an external source to provide compression, shown as lasers in gray, and (b) a high-intensity short-pulse laser to produce relativistic electrons to provide the heating for ignition.

In such an implosion the width of the core is around $20 \mu \mathrm{m}$ and the distance that electrons must travel to reach the core is around $100 \mu \mathrm{m}$. Thus electrons that are accelerated at large angles away from the core will not be able to deposit useful energy for heating.

If we suppose that the electrons at $100 \mu \mathrm{m}$ from a $20 \mu \mathrm{m}$ radius core are a point source with a divergence half angle of $45^{\circ}$, then only around $5 \%$ of the electrons pass through the solid angle of the core. ${ }^{2}$. Since the laser energy to generate electrons is limited, such an efficiency in unacceptable. Therefore the

\footnotetext{
${ }^{2}$ This supposes an isotropic electron divergence for electrons with angles below $45^{\circ}$, giving a solid angle of solid angle is $\pi(2-\sqrt{2})$.
} 
electron divergence should be as low as possible.

An additional consideration is the energy spectrum of these electrons. A $20 \mu \mathrm{m}$ radius core of $300 \mathrm{~g} / \mathrm{cm}^{3}$ means that electrons reaching the core must deposit a majority of their energy within an areal density $\rho z \sim 1 \mathrm{~g} / \mathrm{cm}^{2}$. The collisional energy deposition of electrons within compressed plasma was investigated by Atzeni, et al. ${ }^{[33]}$ This work showed the penetration distance $\mathcal{P}_{90 \%}$ over which electrons deposit $90 \%$ of their energy in the compressed fuel is given by (1.6), where $\mathcal{E}_{0}$ is the electron kinetic energy in $\mathrm{MeV}$ and $\rho$ is the mass density of the fuel. This reduces to the linear relation (1.7) for $\mathcal{E}_{0}$ between 1.5 and $5 \mathrm{MeV}$ at $\rho=300 \mathrm{~g} / \mathrm{cm}^{3}$.

$$
\begin{aligned}
\mathcal{P}_{90 \%}\left[\mathrm{~g} / \mathrm{cm}^{2}\right] & \simeq 0.57 \frac{\mathcal{E}_{0}^{2}}{0.66 \mathcal{E}_{0}+0.34}\left(\frac{\rho}{300 \mathrm{~g} / \mathrm{cm}^{3}}\right)^{0.066} \\
& \simeq 0.7 \mathcal{E}_{0}
\end{aligned}
$$

These relations give penetration distances from 0.2 and $8.2 \mathrm{~g} / \mathrm{cm}^{2}$ for 0.5 and $10 \mathrm{MeV}$ electrons, respectively. An optimal penetration distance is found around $1.5 \mathrm{MeV}$. Electrons with higher energy than this will still deposit energy within the core, but at a lowered efficiency. This exercise show that the electron energy spectrum is also an important consideration in fast ignition research and that electrons in the few $\mathrm{MeV}$ range are desirable.

A final consideration is the efficiency of laser coupling into electrons reaching the cone-tip, which should be maximized due to a limited amount of laser energy. This efficiency consists of two main components, the efficiency of electron acceleration and the fraction of electrons that reach the cone-tip. A major obstacle to this latter component is preplasma filling of the cone. Preplasma comes from material that is ablated by the laser often many nanosecond prior to the main pulse. This can push the laser-interaction surface hundreds of microns ${ }^{[38,39]}$ away from the initial target surface, which dramatically reduces the amount of electron energy reaching the cone-tip. ${ }^{[40,41]}$

Thus, in the cone-guided fast ignition scheme, there are three major characteristics of the electron source that must be understood and optimized to efficiently couple energy into the fuel core. These are the divergence, energy spectrum and 
laser-to-cone-tip coupling efficiency. This dissertation will focus on the latter two topics. The optimization of electron energy reaching the cone-tip will be explored using a laser where the amount of preplasma has been dramatically reduced, leading to electron acceleration occurring much closer to the cone-tip. Concurrently, the energy spectrum accelerated by these lasers will be assessed. Both studies will to generate a quantitative understanding using a combination of experiments and simulations.

\subsection{Studies of Preplasma Effects on Electrons Acceleration}

Fast ignition (FI) research requires high-intensity $\left(>10^{19} \mathrm{~W} / \mathrm{cm}^{2}\right)$, highenergy (>100 J) lasers. Such lasers usually have a finite amount of laser energy, called the prepulse, that proceeds nanoseconds prior to the main pulse. Even if the prepulse is a hundred million $\left(10^{-8}\right)$ times less intense that the main pulse, the intensity is still high energy to ablate a large amount of preplasma. In FI, the cone-walls will contain this ablated preplasma in the lateral dimension and thus the outward extent of the preplasma will be amplified.

The effect of preplasma on accelerated electrons in a cone geometry was observed by VanWoerkom, et al. using electron-induced x-ray emission to image a $\mathrm{Cu}$ cone. ${ }^{[38]}$ The emission from the cone showed that electrons were being generated hundreds of microns away from the inner-tip of the cone, instead of near to the cone-tip as expected in a preplasma-free interaction. This preplasma investigation was progressed by MacPhee et al. again using x-ray imaging to observed the detrimental effect of preplasma. ${ }^{[40]}$ In this work, the energy of the prepulse was intentionally increased to raise the preplasma level. This showed that the peak of x-ray emission was pushed back from $50 \mu \mathrm{m}$ to $200 \mu \mathrm{m}$ by increasing the preplasma energy from 7.5 to $100 \mathrm{~mJ}$. The experimental data was explained in detail using simulations that showed the development of laser filaments within the preplasma; ${ }^{[40,42]}$ an effect that increased substantially at higher levels of preplasma.

During the same period as the work performed by VanWoerkom, et al. ${ }^{[38]}$ 
in cone-targets, Baton, et al. ${ }^{[39]}$ investigated the role of preplasma using cone and cone-like geometries attached to a $\mathrm{Cu}$ foil. In these experiments, the x-ray emission to the $\mathrm{Cu}$ foil was imaged to infer the coupling efficiency and spatial extent of the electrons in the foil. This work showed that a cone-like geometry enhanced the effect of preplasma, which prevented electrons from coupling into the foil. Additionally, this work also showed an encouraging result: by using a laser with extremely low prepulse energy the coupling efficiency could be increased. For this data, the electrons coupling in the foil was identical for the conical and planar targets, indicating that the preplasma was significantly reduced leading to more efficiency electron coupling.

Until this point, the electron coupling decrease due to preplasma had only been evaluated qualitatively. To perform quantitative analysis, work was performed by a group including the author to quantitatively evaluate the spectrum and total energy of electrons passing through the cone-tip. ${ }^{[41,43]}$ The experiment was performed using a $\mathrm{Cu}$ wire attached to the tip of the cone to infer the electron spectrum using the induced x-ray emission from the wire. This showed that by increasing the prepulse energy by a factor of 100x resulted in a 10x decrease in the coupling of energy into the wire. The electron energy spectrum within the wire was inferred using simulations to model the transport of electrons through the cone-wire target. A broad parameter space was studied using injected electron distributions. ${ }^{[43]}$

This study of the disadvantages of preplasma led our group to investigate the potential advantage of reducing the preplasma. The work in this dissertation will expand on our previous work by quantitatively investigating the electron spectrum and coupling when prepulse levels are dramatically reduced. Additionally, an laser-plasma-interaction simulations will allow the electron distribution to be investigated at the fundamental level, while comparing quantitatively to experimental data. 


\subsection{Electron Source Determination and Cone- Wire Targets}

The first work to describe the acceleration of electrons by relativistic intensity lasers was done by Wilks, et al. ${ }^{[44]}$ Here relativistic intensity means that the electrons are traveling at nearly the speed of light and are therefore influenced by both the electric and magnetic field of the laser. One conclusion from this paper was that the kinetic energy of the electrons should be related to the laser intensity $I$ and the laser wavelength $\lambda$ as a function of $\sqrt{I \lambda^{2}}$.

Much work to understand the validity and applicability of this scaling has been performed. Some of the methods used include measurement of accelerated ions from the rear of a target, ${ }^{[45]}$ of electrons that have escaped from the target, ${ }^{[46]}$ and of bremsstrahlung radiation created by electrons passing through a relatively thick target. ${ }^{[6,47]}$

The technique used to measure electron energy spectrum in this dissertation is the imaging of x-rays induced by electrons traveling through a free-standing wire. The entire length of the wire is imaged, allowing the effective path-length of electrons to be measured and used to infer their energy. Previous experiments have used the imaged wire technique and we will build on this knowledge significantly. One might assume that inferring electron spectrum from their effective path-length is a relatively simple inversion problem, considering that the stopping power of electrons in solid-density materials is well known. ${ }^{[48]}$ However, in practice, this is significantly more difficult due to non-linear collective effects (e.g. resistive stopping) within the wire. The reason is that an incredible large amount of charge density and energy is accelerated by the laser. As noted in some of the early studies of these wires, the electrons create strong electric and magnetic fields, ${ }^{[49,50]}$ which dominate the electron transport and make a simple inversion of the path-length unfeasible.

Some of the first attempts to deduce the electron energy spectrum from the $\mathrm{X}$-ray emission from wires ${ }^{[51,52]}$ used simple one-dimensional numerical models. ${ }^{[53]}$ Such models extended simple collisional stopping calculations, as they also included 
self-generated resistive fields created by ohmic inhibition ${ }^{[24]}$ during transport. This approach was expanded using 2D simulations including the self-consistent generation of electric and magnetic fields. ${ }^{[54]}$ The major deficiency in these models was the lack of self-consistent modeling of the vacuum boundary of the wire. In a wire geometry, many electrons escape the target and cause large electric and magnetic fields to be generated along the surface of the wire. The electric potential created on these targets is a few MV. This causes lower energy electrons to be trapped within the wire, but allows the higher energy electrons to escape. Only electrons that stay within the wire contribute to the x-ray emission, thus an accurate modeling of the vacuum boundary is very important. ${ }^{[46]}$

To model the vacuum boundary accurately and to reproduce experimental measurements, our group used the direct-implict particle-in-cell code LSP. ${ }^{[55]}$ Simulations were run in 2D cylindrical geometry and modeled the full-scale geometry of the wire. ${ }^{[41,43]}$ The generation of electric and magnetic fields were included self-consitently using Maxwell's equations and the Lorentz forces to model fields and forces within and outside of the wire. In this work, electrons were injected using an energy spectrum that was varied in average energy until a best fit to the experimental data was produced. Such simulations allowed all features of the wire to be reproduced including the mysterious bump at the end of the wire, which had not previously been understood. ${ }^{[56]}$

In this dissertation, a further step will be taken by modeling the laserinteraction as well as the transport through the wire. These will constrain freeparameters such as the spatial, temporal and angular distributions of the injected electrons. This integrated approach starting from the laser-interaction will be the most comprehensive wire-target modeling to date. Matching experimental data will provide confidence in the accuracy of the simulations, which are then used to explain the acceleration of electrons. 


\subsection{Outline of the dissertation}

Chapter 2 begins with basic plasma concepts and builds upon them to create a broad understanding of the physics present in the laser acceleration of relativistic electrons. The laser interaction physics is discussed, as well as, mechanisms relating to the transport of these electrons through matter.

Chapter 3 is an overview of the laser systems and diagnostics presented in the dissertation. This chapter discusses the spatial and temporal distributions of the lasers, as well as their intensity contrast. An explanation of the $\mathrm{K}_{\alpha} \mathrm{x}$-ray production and measurement is shown. The spectrometer used to measure the total $\mathrm{K}_{\alpha} \mathrm{x}$-ray production and the spherical crystal used to record an x-ray image are described in detail.

Chapter 4 describes direct-implicit particle-in-cell (PIC) modeling. Also included are some of the models used in the simulations and the benchmarking that was done to show that these models are being implemented correctly.

Chapter 5 presents the experimental setup and data taken on the ultra-

high-contrast Trident laser and on the low-contrast Titan laser. The coupling efficiency of electrons into the wire is shown to have a large dependence on the prepulse of the laser and we shown a dramatic increase in coupling efficiency in the high-contrast case. The $\mathrm{K}_{\alpha} \mathrm{x}$-ray images are evaluated by determining the falloff of emission from the initial peak of the wire. This data shows that a the falloff distance is a strong function of the intensity of the laser and that there is a significant difference in falloff between the high and low-contrast cases.

Chapter 6 uses analytical models and rudimentary simulations to develop an intuition of the important physics in the cone-wire targets. Simple models that include the scattering and stopping of electrons are used to understand what energies of electrons will produce $\mathrm{K}_{\alpha}$ x-rays in the wire and which electrons are responsible for the initial falloff. Analytical simulations give a model of how the charging of the wire will occur and what other fields may be present in the system.

Chapter 7 details the use of transport simulations to understand the $\mathrm{K}_{\alpha}$ $\mathrm{x}$-ray profiles observed and to infer electron spectra capable of producing such profiles. The trajectories of electrons traveling through the target and the electron 
energies producing different features of the profile are explained. A large range of injected electron temperatures and total energies are assessed and compared to the experimental data. This is used to estimate electron spectra for the different experimental data points. Finally, the ad-hoc nature of electron injection is discussed and the need for laser simulations addressed.

Chapter 8 discusses the laser-plasma-interaction (LPI) simulations used to model the electron acceleration. The development of the plasma as well as the resulting electron spectrum is discussed in both the high and low-contrast cases. The electrons are resolved in space, time, angle and energy. This allows them to be injected into transport simulations and compared to experimental data. The initial preplasma conditions are assessed and compared to an analytical model. Differences between large scale and small scale preplasma are discussed.

Chapter 9 concludes our work and summarizes the novel and interesting regimes that have been explored.

\subsection{Role of the Author}

The author analyzed the data from all of the diagnostics presented in Chapter 3. This includes the laser focal spot, HOPG spectrometer and crystal imager. In order to obtain quantitative data on the HOPG spectrometer it was calibrated with a single-hit CCD (SHCCD) spectrometer. The author setup and analyzed the SHCCD and HOPG in this calibration and determined the best targets and shot parameters over which to perform the calibration.

The experiments described in Chapter 5 were performed on the Trident Laser at Los Alamos National Laboratory in 2010 and on the Titan Laser at Lawrence Livermore National Laboratory in August 2009. The targets used in these experiments were partially designed by the author. The author managed the assembly of these targets by General Atomics and characterized the targets. The author devised the shot plan at both facilities and decided upon the optimal position of diagnostics within the target chamber. The author optimized the best focus on Titan and assured that the targets were well aligned at both facilities. 
The author did the benchmarking simulations of the electron stopping and scattering described in Chapter 4. Also, the author decided to change the $\mathrm{K}_{\alpha}$ crosssections used in LSP and implemented this change as well. The author developed the analytical models presented in Chapter 6 and ran the simple simulations presented in this chapter. The author performed the transport simulations presented in Chapter 7. The preplasma profile used to simulate the Titan laser was run by S. R. Chawla using the laser description provided by the author. The author ran the laser-plasma-interaction simulations presented in Chapter 8. 


\section{The Physics of Laser Plasma Interactions and Particle \\ Transport}

\subsection{General Laser-Plasma Concepts}

\subsubsection{Shielding and the Debye Length}

Before we can discuss the interaction between lasers, particles and plasma, we must first define the characteristics of a plasma. The defining characteristic of a plasma is that the matter is sufficiently ionized so that the ions and electrons exhibit collective effects governed by their interactions through the electro-magnetic forces. These interactions act to screen out charges and create a quasi-neutral state.

To understand this collective behavior we will start with the simple case of adding a point charge of $Q$ into a plasma at the origin. In this plasma there are electrons and ions, these have a density $n_{\alpha}$, a velocity $v_{\alpha}$, a mass $m_{\alpha}$, and a charge $q_{\alpha}$. Where we use the subscript $\alpha$ to be $e$ for electrons and $i$ for ions. The fluids share a common temperature $T$ and we assume that the plasma has an ionization state of $Z$, thus the electrons and ions have a charge magnitude related to the electron charge $\left|q_{e}\right|$.

The first equation that we use is the momentum equation. This is the fluid equation that relates the forces on the electrons and ions to their acceleration. We have neglected the forces due to magnetic fields, as the electrons are traveling at 
non-relativistic speeds. Thus for electrons,

$$
m_{e} n_{e} \frac{d v_{e}}{d t}=\nabla P_{e}-q_{e} n_{e} E
$$

and ions,

$$
m_{i} n_{i} \frac{d v_{i}}{d t}=\nabla P_{i}+Z q_{e} n_{i} E
$$

We are interested in finding the steady-state solution, so we set the timederivatives to zero. And for the plasma pressure, we assume that the plasma is an ideal gas (i.e. $P_{\alpha}=n_{\alpha} k_{B} T$ ), where the temperature is uniform and isotropic. For the electric field, we use a potential, $\phi$, representation, where $E=-\nabla \phi$. To simplify our derivation, we will only use one-dimension. Thus, for the electrons

$$
0=\frac{\partial}{\partial x} P_{e}-q_{e} n_{e} E=\frac{1}{n_{e}} \frac{\partial n_{e}}{\partial x}+\frac{q_{e} n_{e}}{k_{B} T} \frac{\partial \phi}{\partial x}=\frac{\partial}{\partial x}\left(\log n_{e}+\frac{q_{e} n_{e}}{k_{B} T} \phi\right)
$$

We integrate this equation, using the boundary conditions that at infinity that potential, $\phi$, goes to zero and the density goes to the initial density, $Z n_{0}$. The same can be done for the momentum equation for ions.

$$
\begin{aligned}
& n_{e}=n_{e 0} \exp \left(+\frac{q_{e} \phi}{k_{B} T}\right) \\
& n_{i}=n_{i 0} \exp \left(-\frac{Z q_{e} \phi}{k_{B} T}\right)
\end{aligned}
$$

These equations above are known as the Boltzmann relations. We can see that when a positive charge, $Q$, is applied to a plasma, it pulls electrons towards it and pushes ions away from it. This will cause the charge to be shielded by the plasma. To determine how well this charge is shield, we use the Poisson equation

$$
-\epsilon_{0} \frac{\partial^{2} \phi}{\partial x^{2}}=q_{e}\left(Z n_{i}-n_{e}\right)+Q \delta(x)
$$

Where $\delta(x)$ is the dirac delta function. We now substitute in the Boltzmann relations found above. By assuming that the potential energy is small compared to kinetic energy (i.e. $q_{e} \phi / k_{B} T \ll 1$ ), we can Taylor expand the exponentials (i.e. $e^{x} \approx 1+x$, for small $x$ ) and keep the first order terms.

$$
\begin{aligned}
-\epsilon_{0} \frac{\partial^{2} \phi}{\partial x^{2}} & =q_{e} n_{e 0}\left[\exp \left(-\frac{Z q_{e} \phi}{k_{B} T}\right)-\exp \left(+\frac{q_{e} \phi}{k_{B} T}\right)\right]+Q \delta(x) \\
& =-\frac{(1+Z) q_{e}^{2} n_{e 0}}{k_{B} T} \phi+Q \delta(x)
\end{aligned}
$$


This can be solved by an equation of the form $\phi(x)=A \exp (-x / \lambda)$. We solve for $\lambda(2.8)$ at points away from $x=0$ (i.e. when $\delta(x)=0$ ), and then solve for $A$ by realizing that the plasma is neutral except for the additional charge $Q$ and thus $\int_{-\infty}^{\infty} \phi d x=Q$. This gives the solution:

$$
\phi(x)=Q \exp \left(-x / \lambda_{D}\right)
$$

Where $\lambda_{D}$ is the Debye length given below.

$$
\lambda_{D}=\sqrt{\frac{\epsilon_{0} k_{B} T}{(1+Z) q_{e}^{2} n_{e 0}}}
$$

The Debye length is the length scale over which the plasma becomes neutralized. Thus the requirement that we think of a plama as quasi-neutral is that we are interested in length scales that are greater than the Debye length.

\subsubsection{Plasma Frequency}

We have just described the length-scale over which charge is neutralized. The next step is to describe the time scales over which this charge neutralization occurs. This scale is called the plasma frequency $\omega_{p e}$ and it is the characteristic time scale over which plasma oscillations occur. To derive this, we imagine a plasma where the electrons have been pulled slightly away from the ions, as shown in Figure 2.1.

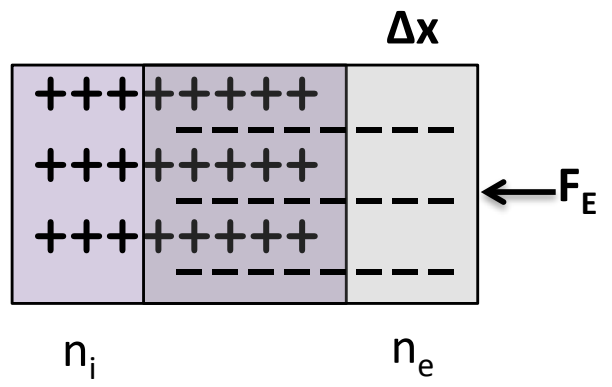

Figure 2.1: Offset of the ion and electron densities used for the derivation of the plasma frequency. 
This offset in densities will create an electric field that can be described by the Poisson equation. We discuss the electric field in the region where the ion density is zero.

$$
\begin{aligned}
-\epsilon_{0} \frac{\partial}{\partial x} E & =q_{e} n_{e} \\
\rightarrow \quad E & =-\frac{q_{e} n_{e}}{\epsilon_{0}} \Delta x
\end{aligned}
$$

To determine the evolution of the plasma, we use the momentum equation, where we neglect the pressure term and substitute in the electric field from the Poisson equation.

$$
0=\frac{d v_{e}}{d t}+\frac{q_{e}}{m_{e}} E=\frac{d^{2} \Delta x}{d t^{2}}-\frac{q_{e}^{2} n_{e}}{m_{e} \epsilon_{0}} \Delta x
$$

This equation, $0=\ddot{x}-\omega^{2} x$, is an ordinary differential equation that gives an oscillating solution with a given frequency, $\omega$. In our case, this frequency is the plasma frequency $\omega_{p e}$.

$$
\omega_{p e}=\sqrt{\frac{q_{e}^{2} n_{0}}{\epsilon_{0} m_{e}}}
$$

This parameter defines the frequency at which electron oscillations occur in the plasma. Essentially, this is the inertia of the plasma electrons in response to an electric field. Which is important to keep in mind, because this determines the plasma response time for given applied field. It shows us the speed at which the plasma can smooth out perturbations in density and fields.

\subsubsection{Propagation of Laser Light Through a Plasma}

Now that we have shown that the plasma can oscillate when introduced to a given perturbation, we can take a look at how the plasma is modified by the electric field of a laser. This interaction will not only affect the plasma, but will also change the propagation of the laser within the plasma.

We begin with a laser that is propagating through the plasma. The electric field of the laser is given by $E=E_{0} \exp [i k z-i \omega t]$. If we begin with the momentum equation for electrons, where we have again neglected the electron pressure.

$$
m_{e} \frac{d v_{e}}{d t}=-q_{e} E
$$


This is integrated to find the electron velocity, and we will substitute in the electron current density $j_{e}=-q_{e} n_{e} v_{e}$.

$$
\begin{aligned}
& v_{e}=\frac{i q_{e} E_{0}}{\omega m_{e}} \\
& j_{e}=-q_{e} n_{e} v_{e}=-\frac{i q_{e}^{2} E_{0}}{\omega m_{e} n_{0}}=-\frac{i \omega_{p e}^{2} \epsilon_{0} E_{0}}{\omega}
\end{aligned}
$$

Now we take the curl of Ampère's law and make a substitution of Faraday's law, after making our substitution for E. Also, we recall that vector identity $\nabla \times$ $(\nabla \times B)=\nabla(\nabla \cdot B)-\nabla^{2} B$. Where we have $\nabla \cdot B=0$ from Maxwell's equations.

$$
\begin{aligned}
\nabla \times(\nabla \times B) & =\nabla \times\left(\mu_{0} j_{e}+\mu_{0} \epsilon_{0} \frac{\partial E}{\partial t}\right) \\
-\nabla^{2} B & =\mu_{0} \epsilon_{0} \nabla \times\left(-\frac{i \omega_{p e}^{2} E}{\omega}+i \omega E\right) \\
& =\frac{1}{c^{2}}\left[-\frac{i \omega_{p e}^{2}}{\omega}+i \omega\right](\nabla \times E)
\end{aligned}
$$

Now we can make the substitutions of Farday's Law (i.e. $\nabla \times E=-\partial B / \partial t$ ) and we take the derivatives.

$$
c^{2} k^{2} B=\left[-\frac{i \omega_{p e}^{2}}{\omega}+i \omega\right](-i \omega B)=\left[-\omega_{p e}^{2}+\omega^{2}\right] B
$$

This gives the dispersion relation for a laser wave in a plasma.

$$
k^{2} c^{2}=\omega^{2}-\omega_{p e}^{2}
$$

Recall that the dispersion relation for a laser in vacuum is simply $k^{2} c^{2}=\omega^{2}$. So we see that the $k$-vector of the laser is decreased by the plasma frequency. The $k$-vector determines how the laser travels in space. Thus when the $k$-vector becomes imaginary, the laser can no longer propagate through the plasma. This occurs when $\omega_{p e}>\omega$. Physically, this means that at this point, the electrons can oscillate at the same frequency as the laser, meaning that the electrons can move fast enough to damp out the laser and prevent it from propagating. So by setting $\omega_{p e}=\omega$ we can determine the density beyond which the laser cannot propagate. This is called the critical density, $n_{c r}$, it is shown below and is expressed as a function of the laser wavelength, $\lambda_{L}$.

$$
n_{c r}=\frac{\epsilon_{0} m_{e} \omega^{2}}{q_{e}^{2}}=\frac{1.1 \times 10^{21}{\mu \mathrm{m}^{2}}^{2}}{\lambda_{L}^{2}} \mathrm{~cm}^{-3}
$$


As the laser impacts the critical density it reflects, but there is a evanescent wave that travels a distance into the material. This can be seen from solving the dispersion equation (2.22) at high densities so that the plasma frequency is much higher than the laser frequency, which gives the solution $k=i \omega_{p e} / c$, which shows that the electric field will falloff exponentially with a characteristic length $\delta_{s}=\omega_{p e} / c$, the skin depth of the plasma (i.e. $E(x)=E_{0} \exp \left[-z / \delta_{s}\right]$ ). This is given by the following equation.

$$
\delta_{s}=\frac{c}{\omega_{p e}}=\sqrt{\frac{2.8 \times 10^{25} \mathrm{~cm}^{-3}}{n_{e}}} \mathrm{~nm}
$$

In this derivation, we have not included relativistic effects. However, as the plasma begins to move at relativistic speeds the inertia of the electrons increases. We take this into account using the relativistic electron mass (i.e. $m_{e} \rightarrow \gamma m_{e}$ ) . Where $\gamma$ is the relativistic factor $\gamma=\left(1-\beta^{2}\right)^{-1 / 2}$, where $\beta=v / c$. Since this increases the electron inertia, they will not be able to accelerate as quickly, thus the density required to damp out the laser will increase. Making this change we get the relativistic plasma frequency, $\omega_{\text {pe* }}$, and the relativistic critical density, $n_{c r *}$.

$$
\begin{aligned}
\omega_{p e *} & =\sqrt{\frac{1}{\gamma}} \omega_{p e} \\
n_{c r *} & =\gamma n_{c r}
\end{aligned}
$$

\subsection{Low-Intensity Interactions}

\subsubsection{Field Ionization}

Previously, we assumed that a plasma already existed to determine some important plasma parameters. Now we will look how this plasma is originally created by a laser. We start by looking at field ionization, where the laser directly excites electrons to leave the atom.

First, we think about the photo-electric effect, where a photon must impart enough energy to an electron to overcome the ionization energy, typically on the order of $10 \mathrm{eV}$. In general, the lasers used in this dissertation are infra-red lasers 
with wavelengths $\sim 1 \mu \mathrm{m}$, which gives a single photon energy of about $1 \mathrm{eV}$, which is below the ionization energy. So how then does this ionization occur? The answer has to do with intensity.

The first process to create ionization as the intenisty is increased is mulitphoton ionization, which is shown graphically in Figure 2.2. Here electrons are still given discrete jumps in energy, as in the photo-electric effect, but there is such a high flux of photons that the electrons do not have time to decay back into the ground state before they are hit by another laser photon. This type of ionization occurs above laser intensities of $10^{11} \mathrm{~W} / \mathrm{cm}^{2}$. [57]

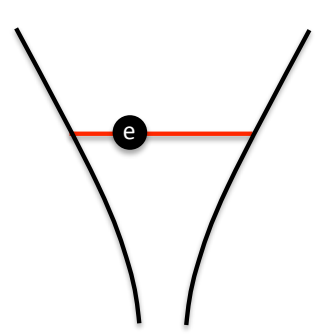

(a) Electron in its ground state in the atom.

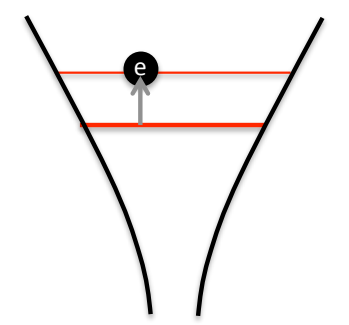

(b) Electron excited by a photon from the laser. The energy gained is not enough to free it from the atom.

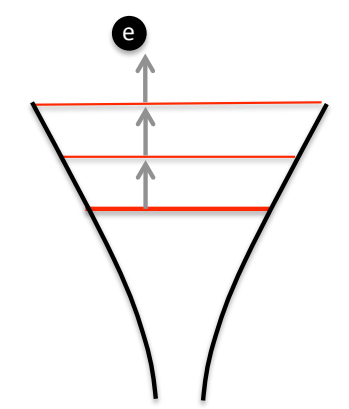

(c) Succesive kicks from the laser photons give the electron enough energy to escape the atom.

Figure 2.2: The mechanism of multi-photon ionization.

At higher intensities, the electric field of the laser is so strong that it can rip electrons from their orbits. The strength of the maximum electric field of a laser is shown below.

$$
I=\frac{\epsilon_{0} c E^{2}}{2}
$$

So a laser intensity of $10^{16} \mathrm{~W} / \mathrm{cm}^{2}$ can create electric fields up to $10^{11} \mathrm{~V} / \mathrm{m}$, which is strong enough to overcome the coulomb potential holding the electron to the atom. A simple model can be used to understand this mechanism. Consider the method followed by Gibbon, ${ }^{[57]}$ where we imagine the Coulomb potential, $\phi$, of the 
atom modified by a constant external electric field, $E$, as shown below.

$$
\phi(x)=\frac{-Z q_{e}^{2}}{x}-q_{e} E x
$$

The electric field bends the potential well to bring it lower, as can be seen in Figure 2.3. To determine the inflection point $\phi_{\text {peak }}$ where the electric field begins to dominate over the potential, we take the differential of the potential and set it to zero (i.e. $d \phi / d x=0$ ). This yields the coordinate of the maximum potential, $x_{\max }=\sqrt{Z q_{e} / E}$. This can then be used in the previous equation and as given in $^{[57]}$ the electric field required to ionize an electron with binding energy $E_{\text {ion }}$ is:

$$
E=\frac{E_{\text {ion }}^{2}}{4 Z q_{e}^{3}}
$$

Which we can also write in terms of intensity, as expressed in Gibbon: ${ }^{[57]}$

$$
I \approx 4 \times 10^{19}\left(\frac{E_{\text {ion }}}{\mathrm{eV}}\right)^{4} Z^{-2} \mathrm{~W} / \mathrm{cm}^{2}
$$

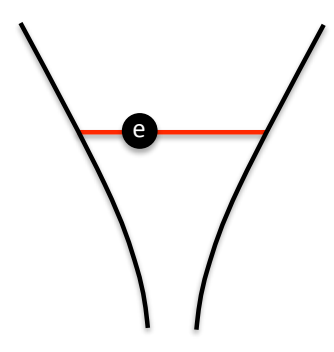

(a) Unmodified potential.

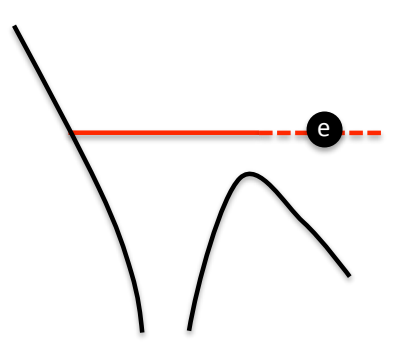

(b) Potential modified by strong electric field.

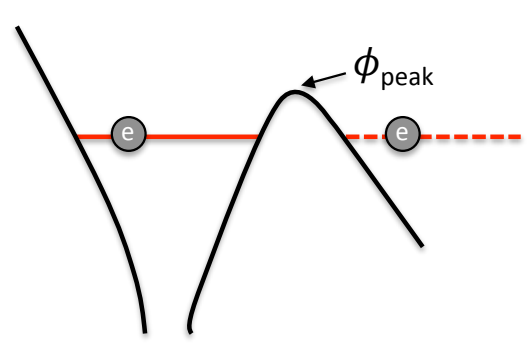

(c) Potential modified by electric field lower than the ionization barrier.

Figure 2.3: Ionization due to modification of the coloumb potential of an atom by an external (laser) electric field.

Thus if we know the ionization potentials for a given material then we can determine the energy required for ionization. A paper by Carlson ${ }^{[58]}$ goes in depth looking at the ionization potentials of many materials at different ionization states. We plot a few materials in Figure 2.4, including the intensity required to achieve 
a given ionization state. This type of ionization is called over-the-barrier (OTB) ionization, because it requires that the electric field of the laser is strong enough to completely overcome the coloumb potential of the atom. However, it is possible to ionize an electron even if the laser electric field is not quite as strong. Essentially, like in OTB, the potential is disturbed by an electric field, but not of sufficient strength to instantly free the electron. However, there is some probability that quantum tunneling will occur. The probability for this to occur was investigated by Ammosov, Delone, and Kraino ${ }^{[59]}$ and is known as ADK ionization.
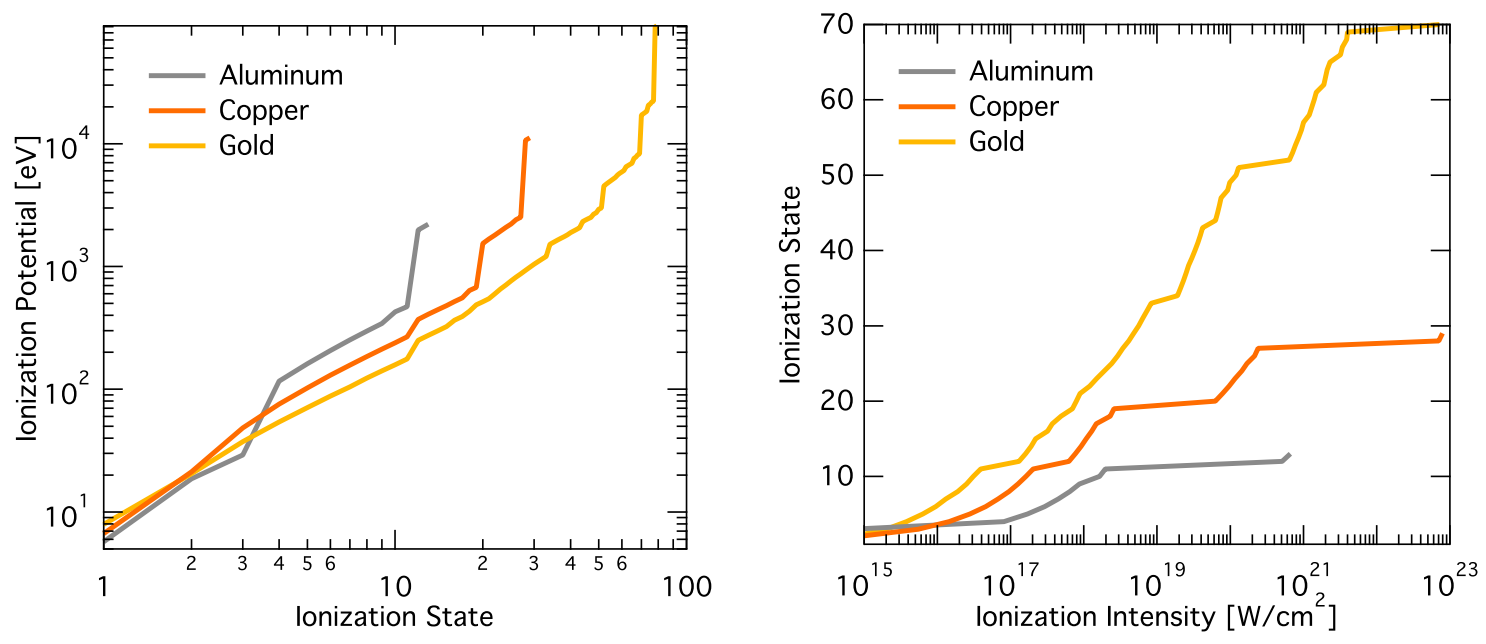

Figure 2.4: Ionization curves for $\mathrm{Al}, \mathrm{Cu}$ and $\mathrm{Au}$, from. ${ }^{[58]}$ The ionization intensity is calculated from equation (2.30).

\subsubsection{Ablation}

So we have seen that lasers can create a plasma simply by stripping electrons from atoms with their strong electric fields. Now we will look into how that plasma expands. So imagine that the laser has ionized a target to an ionization state, $Z$, and heated the plasma to a constant (isothermal) temperature $T$. As before we solve the fluid equations. First, if we assume a quasi-steady-state solution for the electrons (i.e. $\frac{\partial v_{e}}{\partial t}$ vanishes), then we get an electric field of $n_{e} q_{e} E=\partial P / \partial x$. We solve use this electric field in the momentum equation for ions. Here we neglect 
the ion pressure (since the mass of ions is much higher than electrons) and assume a quasi-neutral plasma $\left(n_{e}=Z n\right)$ expanding with velocity $v$. This gives the ion momentum equation (2.31), where we have the ion sound speed $C_{s}=\sqrt{Z k_{B} T / m_{i}}$, and the continuity equation for ions in (2.32).

$$
\begin{array}{r}
\frac{\partial v}{\partial t}+v \frac{\partial v}{\partial x}=-C_{s} \frac{1}{n} \frac{\partial n}{\partial x} \\
\frac{\partial n}{\partial t}+\frac{\partial}{\partial x}(n v)=0
\end{array}
$$

One can find that these equations are solved with a self-similar solution of the form $x / t .^{[60-62]}$ This gives an equation for the expansion velocity of $v=C_{s}+x / t$ and the number density given below, where $n_{0}$ is the solid density.

$$
n=n_{0} \exp \left(-\frac{x}{C_{s} t}\right)=n_{0} \exp \left(-\frac{x}{t} \sqrt{\frac{m_{i}}{Z k_{B} T}}\right)
$$

Thus one can see that both the temperature and ionization state (also affected by temperature) act to increase the scale length of ablated plasma. Additionally, the more time a plasma has to expand, $t$, the longer the scale length can become.

\subsection{High-Intensity Interactions}

\subsubsection{Laser Focusing and Defocusing Mechanisms}

In a plasma, the index of refraction, $\eta$, is equal to $\omega / k$. Using the dispersion relation (2.22) for a laser propagating in a plasma, we get the following.

$$
\eta=\sqrt{1-\frac{\omega_{p e}^{2}}{\omega^{2}}}
$$

If there is a gradient of the index of refraction, this will bend of the light. As seen in Figure 2.5, light tends to bend toward higher $\eta$. This can be used to focus or defocus the light, as in Gradient-index (Grin) optics. This effect is also responsible for visual mirages seen over the desert or polar ice caps, due to large gradients in temperature or density that cause changes in $\eta$. In our case, the index 

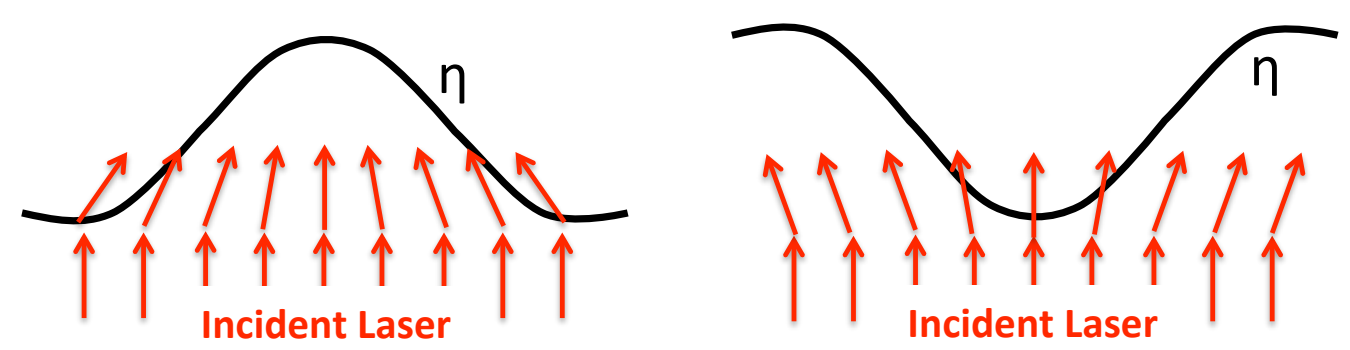

Figure 2.5: Focusing and defocusing of the laser based on changes in the index of refraction, $\eta$.

of refraction is proportional to the plasma frequency, shown here relativistically.

$$
\omega_{\text {pe* }}^{2}=\frac{q_{e}^{2} n_{i}}{\epsilon_{0} m_{e}} \frac{Z}{\gamma_{0}}
$$

Here the electron density is a function of the ion density (i.e. $n_{e}=Z n_{i}$ ). If we assume that the time scales of interest are short compared to the ion motion, then we see that there are gradients in $\eta$ caused by gradients in $Z$. Here an increase in $Z$ that is caused by a higher intensity in the center of the laser spot causes an increase in $\eta$ which is responsible for defocusing the beam. This is called ionization-induced defocusing.

The other gradient of interest is caused by electrons gaining inertia as they become relativistic, as is shown in the average relativistic factor $\gamma_{0}$. This increase in inertia causes the plasma frequency to decrease and will cause focusing of the laser. This mechanism is called relativistic self-focusing.

Thus there are two mechanisms that counter-act each other in the focusing or defocusing of the laser as is illustrated in Figure 2.5. We are interested in knowing which one of these will be dominant for a given laser intensity. To calculate this we take the derivative of $\eta$ with respect to the intensity. Here we are interested in the sign of the derivative to see if the laser will self-focus or de-focus. Since the laser has a higher intensity in the center of the beam, a positive change in $\eta$ will result in focusing and a negative change will cause de-focusing. To make the derivative more simple we look at the derivative of $\eta^{2}$, as this only changes the 
magnitude and not the sign.

$$
\frac{d\left(\eta^{2}\right)}{d I}=\frac{q_{e}^{2} n_{i}}{\epsilon_{0} m_{e}}\left[-\frac{d Z}{d I} \frac{1}{\gamma}+\frac{Z}{\gamma^{2}} \frac{d \gamma}{d I}\right]=\frac{q_{e}^{2} n_{i}}{\epsilon_{0} m_{e}} \frac{Z}{\gamma}\left[-\frac{\partial \log (Z)}{\partial I}+\frac{\partial \log (\gamma)}{\partial I}\right]
$$

Thus we can see that by looking at the differences between the differential logarithms of $Z$ and $\gamma_{0}$ with respect to the intensity, we can determine if the laser will focus or defocus. We use the OTB calculations from Figure 2.4 to determine the differential of $Z$. We use the following equation (2.37), which will be derived later, to give the average relativistic factor of a plasma for a given laser intensity, $I$ and wavelength, $\lambda$.

$$
\gamma_{0}=\sqrt{1+\frac{I \lambda^{2}}{1.37 \times 10^{18} \mathrm{~W} / \mathrm{cm}^{2}}}
$$

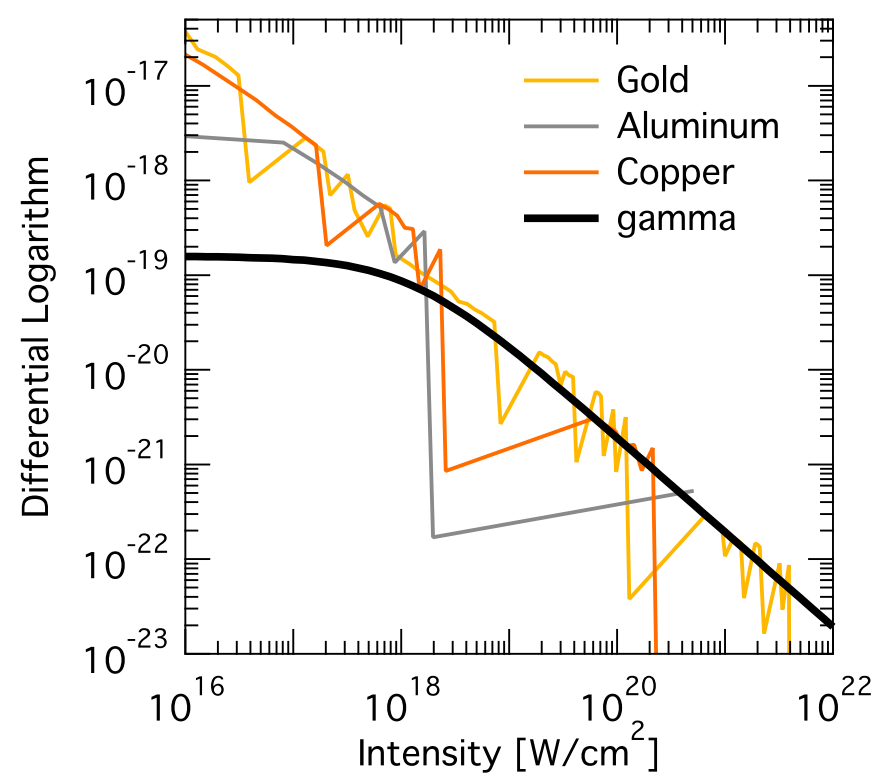

Figure 2.6: Plot of differential logarithms of $Z, \frac{\partial \log (Z)}{\partial I}$, and $\gamma_{0}, \frac{\partial \log \left(\gamma_{0}\right)}{\partial I}$, for given intensities.

The values of these differential logaritms are plotted in Figure 2.6. These plots shows quite uneven behavior for the ionization state $Z$ due to the simple OTB model that we used assuming that only one ionization state exists at a time. In reality this curve would be much smoother and we should be careful about using 
it for quantitative assessments. However, interesting trends can still be seen. For instance, in Figure 2.6, we see that the ionization change is certainly dominant for intensities below $10^{18} \mathrm{~W} / \mathrm{cm}^{2}$, when the plasma can be considered non-relativistic. However, above $10^{18} \mathrm{~W} / \mathrm{cm}^{2}$ the change in $\gamma$ becomes more important. Since these calculations are dependent on the discrete atomic transitions it is difficult to determine if the $\gamma$ of $Z$ dependence will be dominant in Au. However, it seems that there is a large difference in the $Z$ dependence of gold versus copper or aluminum. Here the latter two materials saturate, as they cannot ionize above a certain amount. Thus we may expect a difference in the self-focusing between these lower atomic number elements and gold.

Another mechanism that can occur due to these changes in $\eta$ is filamentation. Note that in the case where we have relativistic self-focusing, it is a nonlinear mechanism that grows on itself with positive feedback. As the higher intensity causes the beam to focus, this enhanced focus causes higher intensity, which continues to feed back on itself. When such positive feedback occurs, small perturbations cause non-linear instabilities. These instabilities create filaments, where the laser splits up into multiple portions that each self-focus independently.

\subsubsection{Ponderomotive Force and Hole Boring}

Over longer time scales, a mechanism called the ponderomotive force can cause motion of the plasma density over larger time scales. This force is caused by gradients in the intensity of the laser. Electrons are pushed harder by more intense parts of the laser and less hard by lower intensity portions. Thus as electrons oscillate across these intensity gradients they are pushed away from the areas of high intensity and inevitably drag the ions along with them.

We can look at a single electron in a laser, where we describe the electric field as $E=E_{0} \cos \phi=E_{0} \cos (k z-\omega t)$. We assume that the spatial variation in laser intensity is small compared to the spatial variation of its oscillations. Then we integrate the Lorentz force equation for a single electron for the non-relativistic 
case (i.e. no magnetic field force).

$$
\begin{aligned}
\frac{\partial v}{\partial t} & =-\frac{q_{e}}{m_{e}} E_{0} \cos \phi \\
\rightarrow \quad v & =\frac{q_{e}}{m_{e} \omega} E_{0} \sin \phi \\
\rightarrow \quad x & =-\frac{q_{e}}{m_{e} \omega^{2}} E_{0} \cos \phi
\end{aligned}
$$

Now that we have an equation to determine the position of the particle, we introduce the change of the electric field across space. We again assume that the spatial change is slowly varying, this allows us use a linear approximation of $E(x)=E_{0}+x \partial E_{0} / \partial x$. What we want to understand is not the quick motion of the electrons, but rather the long time-scale motion of the electrons over many laser cycles. To understand this, we take the time averaged force on the electrons. This force is the ponderomotive force $F_{p}$.

Here $\langle y(t)\rangle_{t}$ denotes the time average of some variable $y(t)$. We have only taken the second order terms into account and we recall that $\partial y^{2} / \partial x=2 y \partial y / \partial x$. Also, we use equation (2.27) to transform the electric field into an intensity, $I_{0}$.

$$
\begin{aligned}
F_{p}=\left\langle m \frac{\partial v}{\partial t}\right\rangle_{t} & =-q_{e}\left\langle E_{0} \sin (\omega t)+\frac{q_{e}}{m \omega^{2}} E_{0} \frac{\partial E_{0}}{\partial x} \sin ^{2}(\omega t)\right\rangle_{t} \\
& =-\frac{q_{e}^{2}}{4 m \omega^{2}} \frac{\partial E_{0}^{2}}{\partial x} \\
& =-\frac{q_{e}^{2}}{2 m \omega^{2}} \frac{1}{\epsilon_{0} c} \frac{\partial I_{0}}{\partial x}
\end{aligned}
$$

As described before, we see that the higher intensity regions of the laser tend to push electrons away. Over time this can create channels of low density plasma and is sometimes called hole-boring.

\subsection{3 $\mathrm{j} \times \mathrm{B}$ Acceleration Mechanism}

Now that we have looked at the electron motion in the non-relativistic case, we want to look at what happens when the electrons are accelerated to relativistic velocities. Again, we will have a plane polarized electro-magnetic wave, where the 
electric field is in the $x$-direction and the wave propagates in $z$.

$$
\begin{aligned}
& E \hat{\mathbf{x}}=E_{0} \cos \phi \hat{\mathbf{x}}=E_{0} \cos \left(\frac{\omega}{c} z-\omega t\right) \hat{\mathbf{x}} \\
& B \hat{\mathbf{y}}=\frac{E_{0}}{c} \cos \phi \hat{\mathbf{y}}
\end{aligned}
$$

Now we must solve the Lorentz force equations in the $x$ and $z$ directions (as $y$ is not acted upon by any forces, we can ignore it).

$$
\begin{aligned}
& \frac{d p_{x}}{d t}=-q_{e}\left(E-v_{z} B\right) \\
& \frac{d p_{z}}{d t}=-q_{e}\left(v_{x} B\right)
\end{aligned}
$$

We first notice that the following equations are true:

$$
\begin{aligned}
-\frac{E_{0}}{\omega} \frac{\partial}{\partial t}[\sin (k z-\omega t)] & =E_{0} \cos (k z-\omega t)=E_{x} \\
\frac{E_{0}}{\omega} \frac{\partial}{\partial z}[\sin ((\omega / c) z-\omega t)] & =\frac{E_{0}}{c} \cos (k z-\omega t)=B_{y}
\end{aligned}
$$

Then these equations can be substituted into the force equation for $x$. Here we also recall the definition of the full derivative of $y(z, t)$ as $d[y(z, t)] / d t=\partial y / \partial t+$ $v_{z}(\partial y / \partial z)$. Then we integrate.

$$
\begin{aligned}
\frac{d p_{x}}{d t} & =\frac{q_{e} E_{0}}{\omega}\left(\frac{\partial}{\partial t} \sin \phi+\frac{\partial z}{\partial t} \frac{\partial}{\partial z} \sin \phi\right) \\
& =\frac{q_{e} E_{0}}{\omega} \frac{d}{d t}(\sin \phi) \\
\rightarrow \quad p_{x} & =\frac{q_{e} E_{0}}{\omega} \sin \phi+C_{0 x}=\frac{q_{e} E_{0}}{\omega} \sin \phi
\end{aligned}
$$

Here we end up with a constant of integration $C_{0 x}$. We are interested in finding the solution where the time average of the $p_{x}$ is zero, thus we get $C_{0 x}=0$.

Next, we multiply together the Lorentz equations in $x$ and $z$ and rearrange. We note that $\frac{d}{d t} y^{2}=2 y \frac{d}{d t} y$ and that the total momentum $p_{T}$ is the quadrature sum of $p_{x}$ and $p_{z}$.

$$
\begin{aligned}
-q_{e} E_{x}\left(1-\frac{1}{c m \gamma} p_{z}\right) \times \frac{d p_{z}}{d t} & =-q_{e} E_{x}\left(\frac{1}{c m \gamma} p_{x}\right) \times \frac{d p_{x}}{d t} \\
\frac{d p_{z}}{d t} & =\frac{1}{c m \gamma}\left(\frac{d p_{x}^{2}}{d t}+\frac{d p_{z}^{2}}{d t}\right) \\
& =\frac{1}{c m \gamma} \frac{d p_{T}^{2}}{d t}
\end{aligned}
$$


The relativistic energy-momentum relationship is $p_{T}^{2}=\mathcal{E}^{2} / c^{2}-(m c)^{2}=m^{2} c^{2}\left(\gamma^{2}-\right.$ 1) and therefore

$$
\frac{d}{d t} p_{T}^{2}=m^{2} c^{2} \frac{d}{d t}\left[\gamma^{2}\right]=2 \gamma m^{2} c^{2} \frac{d \gamma}{d t}
$$

Substituting this into (2.55), we obtain

$$
\begin{aligned}
\frac{d p_{z}}{d t} & =m c \frac{d \gamma}{d t} \\
p_{z} & =m c \gamma+C_{0 z}
\end{aligned}
$$

As previously for $p_{x}$, we want to find a solution where the momentum of $p_{z}$ has a time average of zero. However, this corresponds to a case where the timeaveraged kinetic energy is not zero. Instead, the time-average of $\gamma$ is $\langle\gamma\rangle_{t}=\gamma_{0}$, which gives $C_{0 z}=-m c \gamma_{0}$. And thus:

$$
p_{z}=m c\left(\gamma-\gamma_{0}\right)
$$

Now that we have the relationship of the momentum with $\gamma$, we wish to determine how $\gamma$ varies with the intensity of the laser. To do this, we again use the energy-momentum relationship $p_{T}^{2}=m^{2} c^{2}\left(\gamma^{2}-1\right)$, and then substitute in the components of the momentum.

$$
\begin{aligned}
\gamma^{2}-1 & =\left(\frac{p_{x}}{m c}\right)^{2}+\left(\frac{p_{z}}{m c}\right)^{2} \\
& =\left(\frac{q_{e} E_{0}}{\omega m c} \sin \phi\right)^{2}+\left(\gamma-\gamma_{0}\right)^{2}
\end{aligned}
$$

We define the normalized amplitude of the laser as $a_{0} \equiv q_{e} E_{0} /(\omega m c)$ giving

$$
\begin{aligned}
a_{0}^{2} \sin ^{2} \phi+\left(\gamma-\gamma_{0}\right)^{2} & =\gamma^{2}-1 \\
a_{0}^{2} \sin ^{2} \phi & =2 \gamma \gamma_{0}-\gamma_{0}^{2}-1
\end{aligned}
$$

Now we take the time average and recall that $\left\langle\sin ^{2} \phi\right\rangle_{t}=1 / 2$ and $\langle\gamma\rangle_{t}=\gamma_{0}$. After doing this time averaging, we get an equation that relates the averaged relativistic gamma to the normalized intensity of the laser.

$$
\gamma_{0}=\sqrt{1+\frac{a_{0}^{2}}{2}}
$$


Since the kinetic energy, $\mathcal{E}_{k}=(\gamma-1) m c^{2}$. We can use this to get the time-averaged kinetic energy.

$$
\mathcal{E}_{k}=m c^{2}\left[\sqrt{1+\frac{a_{0}^{2}}{2}}-1\right]=0.511 \mathrm{MeV}\left[\sqrt{1+\frac{I \lambda^{2}}{1.37 \times 10^{18} \mathrm{~W} / \mathrm{cm}^{2}}}-1\right]
$$

This is the well-known ponderomotive potential of the laser. As we have just shown, this is the average kinetic energy that an electron will have in a linearly polarized electro-magnetic wave.

We can again arrange the relativistic energy-momentum relationship to determine the motion of the electron in $z$. We will use the trigonometric relationship of $2 \sin ^{2} \phi=1-\cos 2 \phi$.

$$
\begin{aligned}
\gamma^{2}-1 & =\left(\frac{p_{x}}{m c}\right)^{2}+\left(\frac{p_{z}}{m c}\right)^{2} \\
& =a_{0}^{2} \sin ^{2} \phi+\left(\frac{p_{z}}{m c}\right)^{2}=\left(\frac{p_{z}}{m c}\right)^{2}+2 \frac{p_{z}}{m c} \gamma_{0}+\gamma_{0}-1 \\
\frac{p_{z}}{m c} & =\frac{1}{2 \gamma_{0}}\left[-\frac{a_{0}^{2}}{2} \cos 2 \phi+\frac{a_{0}}{2}+1-\gamma_{0}^{2}\right] \\
& =-\frac{a_{0}^{2}}{4 \gamma_{0}} \cos 2 \phi
\end{aligned}
$$

So now we can write out the momentum equations in both directions.

$$
\begin{aligned}
\frac{p_{z}}{m c} & =-\frac{a_{0}^{2}}{4 \gamma_{0}} \cos 2 \phi \\
\frac{p_{x}}{m c} & =a_{0} \sin \phi
\end{aligned}
$$

We see that in this case the time-averaged momentum is zero in both directions. Thus when oscillating in this laser field, the electron will gain any energy over time. However, because the electrons are oscillating in the $z$ direction, they can be accelerated over any gradients in $z$. For instance, if the laser is near to the critical density, then the laser will accelerate electron up to the critical density. But then, once these electrons are pushed passed critical density, the laser cannot propagate and the electrons are not pulled back by the laser, thus they are pushed into the materials.

This acceleration mechanism is called $\mathbf{j} \times \mathbf{B}$ heating, because electrons are able to gain momentum in the laser propagation direction due to their relativistic 


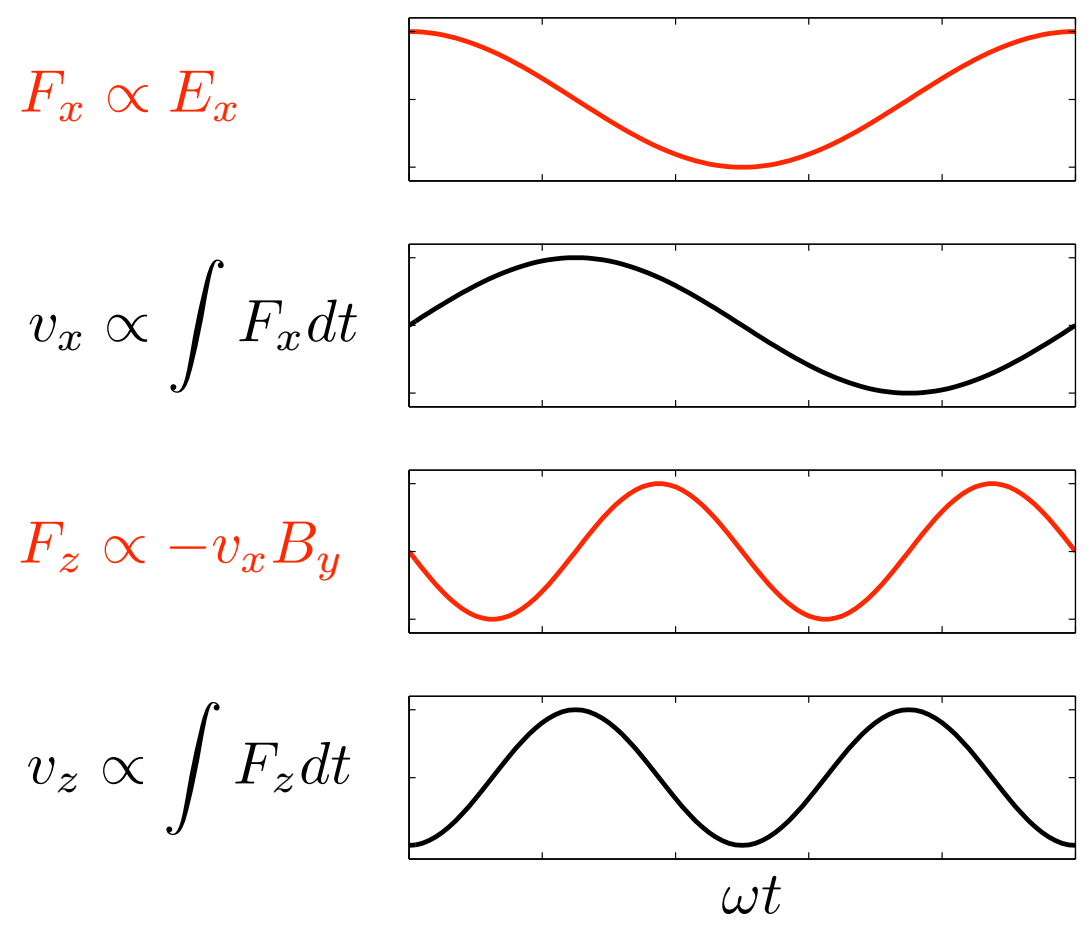

Figure 2.7: Motion of electrons and forces in a relativstic laser field.

$\mathbf{v} \times \mathbf{B}$ motion. This story can be shown through the explanatory graphic in Figure 2.7. First the electron is pushed by the electric field, it gains velocity in the $x$ direction as it is pushed by this field. Then, as it increases in $x$-velocity it is acted upon by a force in $z$ from the cross-product of $v_{x}$ and $B_{y}$ which causes an acceleration in $z$. As both $v_{x}$ and $B_{y}$ are sinusoidally varying terms their product has double the frequency of the original wave (i.e. $2 \omega)$. Thus we note that the acceleration of electrons due to $\mathbf{j} \times \mathbf{B}$ heating occurs at twice the laser frequency.

Another important part of this acceleration mechanism is the angle at which the electrons are accelerated. This can be determined by dividing determining the ratio of the momentum in the forward and transverse directions. We can first find $p_{x}^{2}=m^{2} c^{2}\left(2 \gamma \gamma_{0}-\gamma_{0}^{2}-1\right)$ from (2.59) and the momentum-energy relation. Thus the ejection angle is

$$
\tan \theta=\frac{p_{x}}{p_{z}}=\sqrt{\frac{2 \gamma \gamma_{0}-\gamma_{0}^{2}-1}{\left(\gamma-\gamma_{0}\right)^{2}}}
$$


For an electron starting at rest $\gamma_{0}=1$, which gives the following:

$$
\tan \theta=\sqrt{\frac{2}{\gamma-1}}
$$

This equation shows that higher energy electrons are ejected at smaller angles from the laser-direction, as compared to lower energy electrons.

\subsection{Electron Transport Physics}

Now that we have discussed how relativistic electrons can be accelerated by lasers due the $\mathbf{j} \times \mathbf{B}$ force, we look into what happens to them after they are accelerated. In our discussion of the laser and plasma interactions, we could expect that the materials would behave like plasmas. However, in the case of electron transport, the electrons are often traveling through materials at solid density that do not necessarily behave like a plasma. Thus we must take into account effects that occur in cooler, denser matter, such as electron-ion collisions, as well as the collective effects that occur in plasma.

\subsubsection{Collisional Effects and Plasma Resistivity}

\section{Spitzer Resistivity}

We begin by looking at collisions between electrons and ions. Consider the case of an electron at an impact distance $r$ from an ion of charge $Z q_{e}$ with a momentum $p=m_{e} v$. The force, $F$ at the closest approach will be

$$
F=m \frac{d v}{d t} \approx-\frac{Z q_{e}^{2}}{4 \pi \epsilon_{0} r^{2}}
$$

For this estimate we assume that the force is constant and occurs only over the time $t=r / v$, which gives a change in momentum of $\Delta v=F(r / m v)$. So we now have an understanding for how the electron interacts after one collision. However, we are interested in how the electrons move after multiple collisions with ions, and we'd like to know the time scale over which this occurs. Naively one might think 


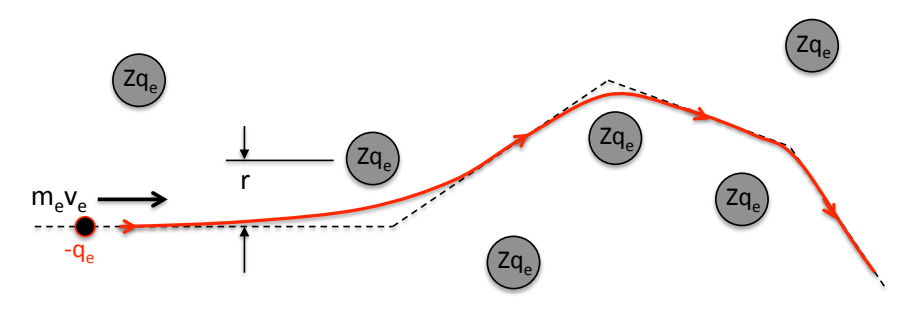

Figure 2.8: Electron being scattered off multiple ions resulting in a random walk like movement.

that this could be found by taking the time derivative of $\Delta p$. However, the fact that the electron can scatter in multiple directions and that each scattering event is unique, means that this is a random-walk like interactions. Thus we take the time derivative of the the square of the change in momentum (i.e. $\left.(\Delta p)^{2}\right)$. To do this we multiple this value by the velocity of the electrons $v$ the density of the ions $n_{i}$ and then we must integrate over all impact parameters $\pi \int r d r$ to find the average momentum change.

$$
\frac{d}{d t}(\Delta p)^{2}=n_{i} \int_{r_{\min }}^{r_{\max }} r\left(-\frac{Z q_{e}^{2}}{4 \pi \epsilon_{0} r v}\right)^{2} d r=\frac{Z^{2} q_{e}^{4} n_{i}}{16 \pi \epsilon_{0}^{2} v^{2}} \int_{r_{\min }}^{r_{\max }} \frac{d r}{r}=\frac{Z^{2} q_{e}^{4} n_{i}}{16 \pi \epsilon_{0}^{2} v^{2}} \ln \Lambda
$$

As can be seen from above, $\ln \Lambda$ is the ratio of the maximum to the minimum impact parameters. The maximum impact parameter is the Debye length, because this is the maximum range of fields in a plasma (as shown previously). The minimum impact parameter is set by the larger of the de Broglie wavelength and the classical distance of closest approach. To determine the time at which the random-walk momentum is equivalent to the initial momentum, we divide the above equation by $p=m v$ and take the derivative of both sides. However, we are really interested in the electron-ion collision frequency $\nu_{e i}$, which is the inverse of this time.

$$
\nu_{e i}=\frac{Z^{2} q_{e}^{4} n_{i}}{16 \pi \epsilon_{0}^{2} m v^{3}} \ln \Lambda
$$

We use the Drude model, which says that the resistivity $\eta$ of a material is proportional to the inverse of the electron inertia times the collision frequency. Also, to make this applicable to an equilibrium plasma, we can take the use the 
average velocity of a Maxwellian distribution $v_{a v g}=\sqrt{k_{B} T_{e} / m}$. This gives the Spitzer Resistivity of a plasma. ${ }^{[63]}$

$$
\eta=\frac{m_{e}}{n_{e} q_{e}^{2}} \nu_{e i}=\frac{Z q_{e}^{2} m_{e}^{1 / 2} \ln \Lambda}{16 \pi \epsilon_{0}^{2}} \frac{1}{\left(k_{B} T_{e}\right)^{3 / 2}}
$$

\section{LMD Resistivity}

As before, the Spitzer model offers a plasma physics based interpretation of scattering. One can see that, as the temperature of the plasma goes to zero, the resistivity becomes infinite. If this were true, then there would be no conductors at room temperature, which is certainly not the case. Thus to understand the resistive behavior at lower energies, we consider the Lee and More model ${ }^{[64]}$ with Desjarlais corrections, ${ }^{[65]}$ known as LMD. This model includes the material behavior at temperatures where inter-atomic forces are more relevant than plasma parameters. However, the model still reproduces the $\left(k_{B} T_{e}\right)^{-3 / 2}$ behavior of Spitzer as the material temperature increases and becomes more plasma like. The Figure 2.9 shows plots of the resistivity of three different materials across a broad range of temperatures, which are of interest to this work.

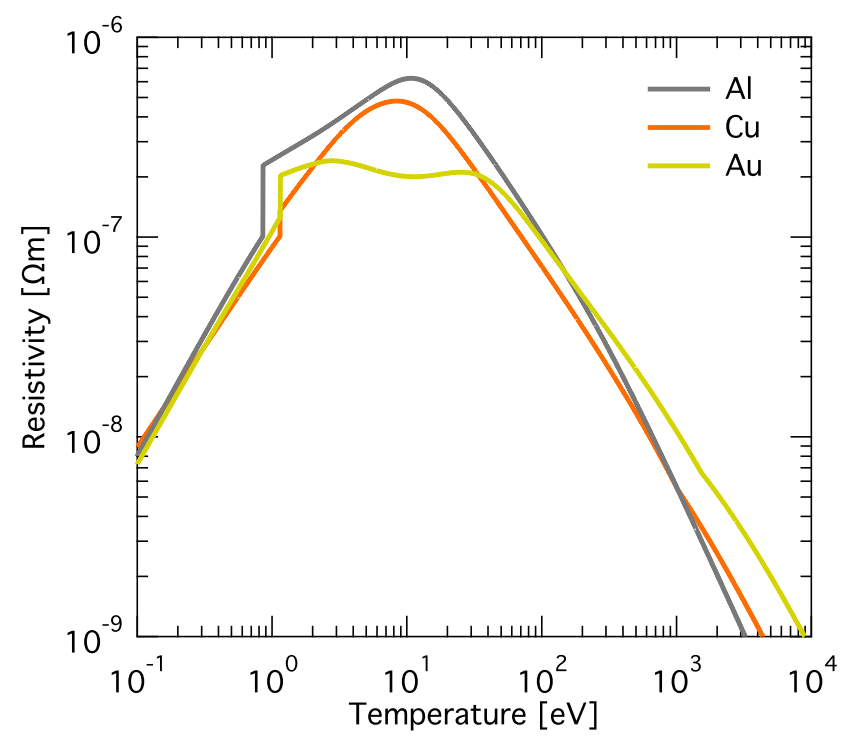

Figure 2.9: Resistivity curves for $\mathrm{Al}, \mathrm{Cu}$ and $\mathrm{Au}$ determined using the LMD resistivity model. 


\subsubsection{Alfvén Current Limit}

Now that we have discussed the resistivity created by rather low energy energy electrons transporting through plasma, we move to an extremely different case: a high-energy, large current electron beam propagating through vacuum. We use this to calculate the Alfvén current limit. This is the largest net current that can physically be sustained. Here we start by supposing a current $I_{0}$ propagating through vacuum with a radial extent of $R$. Driving a current generates a magnetic field from Ampère's law. Here we suppose a steady state solution and use the Biot-Savart law to determine the strength of the magnetic field $B$ at the edge of the beam.

$$
B=\frac{\mu_{0} I_{0}}{2 \pi R}
$$

Now let us think of a single electron in that beam at the radius $R$ from the center. This electron will be driven in a cyclotron motion. The radius of this motion is given by the Larmor radius $R_{L}=p /\left(q_{e} B\right)$, where $p$ is the electron's momentum. Now the case for this electron to be completely turned around by the magnetic field is when the Larmor radius is smaller than twice the radius of the starting point of the electron (i.e. $R<2 R_{L}$ ). So if we plus this into the above equation we can solve for the current necessary to perform this reversal of the electron.

$$
I_{\text {Alfvén }}=\frac{4 \pi}{\mu_{0} q_{e}} p=\frac{4 \pi m_{e} c}{\mu_{0} q_{e}} \gamma \beta
$$

This simple argument is actually the derivation for the Alfvén current limit, ${ }^{[23]}$ though it can be done in much more detail. ${ }^{[66,67]}$ This is the current at which the self-generated magnetic field of the current causes the beam to pinch and reverse on itself. For an electron with $1 \mathrm{MeV}$ of kinetic energy, this gives a maximum current of less than $50 \mathrm{kA}$, even $10 \mathrm{MeV}$ electrons are Alfvén-limited at $350 \mathrm{kA}$. However, mega-Amp currents have been observed experimentally in short-pulse laser experiments and are, indeed, necessary for fast ignition. So how are these currents possible? The answer is that the magnetic field growth is dependent on the net current, not simply the forward streaming current. Thus, the 
fact that high currents have been observed hints at a neutralizing current that is drawn from the background plasma and capable neutralizing the forward current.

\subsubsection{Return Current Response Time}

From the case of a fast current propagating in vacuum, we move to the case of a current propagating in a plasma. This forward (or fast) current $j_{f}$, will draw a background current $j_{b}$ due to the electric field that the current creates as it propagates through the plasma. We look at the time necessary to draw this background current and thus to prevent the magnetic field from collapsing the forward current.

We begin with the momentum equation for the background electrons. As the background electrons should stay non-relativistic, we have neglected the magnetic forces. However, we have added an additional force from the collisions of the background current within the plasma. This is modeled by the resistivity $\eta$. As we discussed previously, the resistivity is proportional to the electron-ion collision frequency of the plasma $\nu_{e i}=\eta n_{0} q_{e}^{2} / m_{e}$. We can rearrange the equation to get a solution for the electric field, $E$.

$$
\begin{aligned}
\frac{m_{e}}{q_{e}^{2} n_{0}} \frac{d j_{b}}{d t} & =-E-\eta j_{b} \\
\rightarrow \quad E & =-\frac{m_{e}}{q_{e}^{2} n_{0}} \frac{d j_{b}}{d t}-\eta j_{b}
\end{aligned}
$$

This solution for the electric field can now be inserted into Ampère's law. In this simple estimate we neglect the magnetic field (i.e. $\nabla \times B \rightarrow 0$ ).

$$
\begin{aligned}
0 & =-\epsilon_{0} \frac{d E}{d t}+j_{b}+j_{f} \\
& =\frac{m_{e} \epsilon_{0}}{q_{e}^{2} n_{0}} \frac{d^{2} j_{b}}{d t^{2}}+\eta \epsilon_{0} \frac{d j_{b}}{d t}+j_{b}+j_{f} \\
& =\frac{d^{2} j_{b}}{d t^{2}}+\nu_{e i} \frac{d j_{b}}{d t}+\omega_{p e}^{2} j_{b}+\omega_{p e}^{2} j_{f}
\end{aligned}
$$

Notice that this equation has the form of a driven-damped harmonic oscillator. This gives a solution of the form $j_{b}=A_{0} \exp \left(\omega_{b g} t\right)+B_{0}$, where $-\omega_{b g}$ is the complex decay frequency of the oscillator. By inserting this solution into the equation 
using the boundary conditions of the problem, this gives $j_{b}=-j_{f}\left[1-\exp \left(-\omega_{b g} t\right)\right]$ as the solution. In our case, we are interested in the decay time, $\omega_{b g}$.

$$
\omega_{b g}=\frac{\nu_{e i}-\sqrt{\nu_{e i}^{2}-4 \omega_{p e}^{2}}}{2}=\frac{\nu_{e i}}{2}\left[1-\sqrt{1-4 \frac{\omega_{p e}^{2}}{\nu_{e i}^{2}}}\right]
$$

As this is a harmonic oscillation, there are three different regions of interest that can be defined by the so-called damping ratio, $\zeta=\nu_{e i} /\left(2 \omega_{p e}\right)$, which determines the type of response that will dominate the solution. When $\zeta<1$ (i.e. $\left.\omega_{p e}>\nu_{e i}\right)$ this is the underdamped solution. In this case, the solution will be have a complex frequency, indicating the there are sinusoidal fluctuations. When the electron-ion collision frequency is negligible compared to the plasma frequency, the frequency of these oscillations will give an imaginary frequency of $\omega_{b g}=i \omega_{p e}$, which corresponds to plasma waves being driving by the fast current. These oscillations should be able to neutralize the fast current it they are faster than the characteristic time of this current. It is intuitive to the think of the plasma frequency as the inertia of the electrons in the plasma, because it is essentially the acceleration time of these electrons.

The next important region of interest is the critical damping, where $\zeta=1$. This is the maximum damping time, which the decay time is $\omega_{b g}=\nu_{e i} / 2$ and occurs when the plasma frequency is half of the electron-ion collision frequency.

As the damping ratio increases, $\zeta>1$, the plasma becomes over-damped. This means that the collisional time is now so fast that the plasma is damped before oscillations can occur. Thus, at this point, the plasma cannot support a fast current that is on the order of the plasma frequency timescale. Interestingly, as the plasma increases in density, the plasma response time equilbrates to a given value. This value can be found through a Taylor expansion of the determinant (i.e. $\sqrt{1+x} \approx 1+x / 2$ ). This gives a value of $\omega_{b g}=\omega_{p e}^{2} / \nu_{e i}=\epsilon_{0} / \eta$. Thus, at high densities, we see that the response time is dependent on the resistivity of the plasma alone. 


\subsubsection{Current Neutrality Conditions}

So now we have an understanding of the timescale of the drawing of the background return current. Now what do we compare this to? One might first think that it would be appropriate to compare the timescales to cyclotron frequency, because this is the amount of time that it would take an electron to turn around in a given magnetic field. However, this magnetic field must already be developed, which means that the return current was not drawn at a quick enough pace to neutralize the fast current.

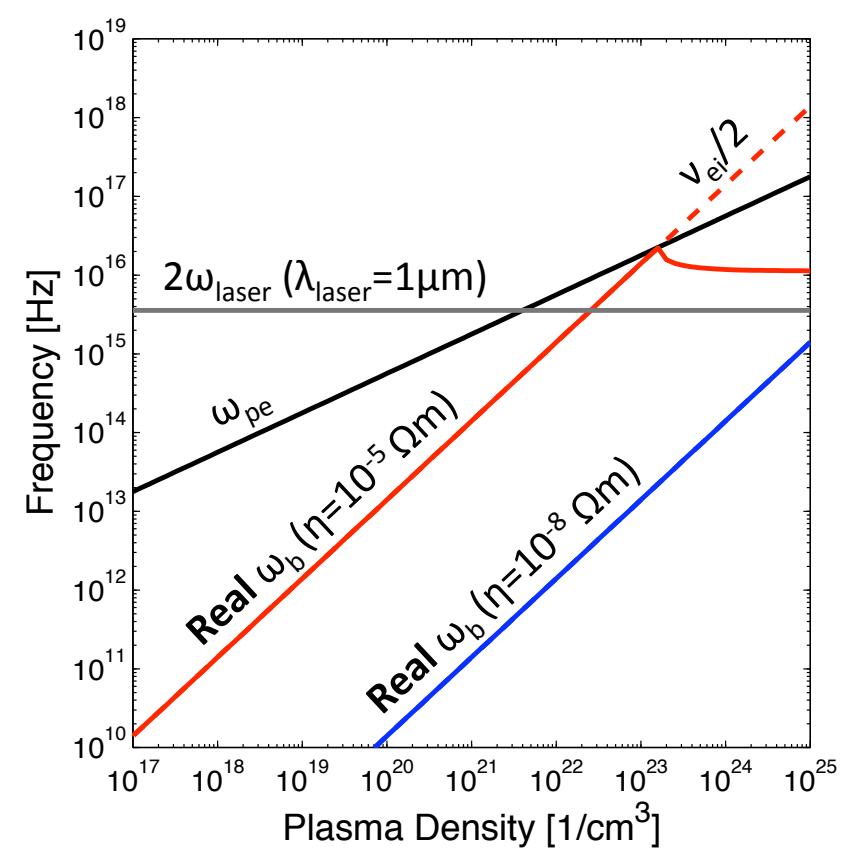

Figure 2.10: Plot of timescales of interest in return current generation. The laser frequency, $\omega_{\text {Laser }}$, is shown for a $1 \mu \mathrm{m}$ laser. The plasma frequency $\omega_{p e}$ is plotted. Return current decay time is plotted for two cases 1) for high value of resistivity $10^{-5} \Omega \mathrm{m}$ and 2 ) for metals at room temperature $10^{-8} \Omega \mathrm{m}$.

Instead, a better number for the fast current timescale, is the laser frequency $\omega_{L}$. Because, as we can recall from the previous section on $\mathbf{j} \times \mathbf{B}$ acceleration, the fast electrons are injected at a rate of twice the laser frequency. Thus this is the best comparative timescale. Electrons are injected near the critical density and this density corresponds to the frequency of the laser, thus from a laser accelera- 
tion perspective, we expect the plasma frequency to compensate the fast electron current in almost every case.

Figure 2.10 shows the frequency timescales of a interest to the return current generation. The plasma frequency, $\omega_{p e}$ is plotted to show the timescale at which the plasma waves can move to shield the fast current, as well as twice the laser frequency to show the fast current timescales. The electron-ion collision frequency $\nu_{e i}$ is shown for two cases. The case of resistivity of $10^{-5} \Omega \mathrm{m}$ is a high estimate for the peak of resistivity for a plasma and the case of $10^{-8} \Omega \mathrm{m}$ is consistent with metals at room temperature. One can see that even for quite high resistivities (i.e. $10^{-5} \Omega \mathrm{m}$ ) that the effect of collisions will most likely not play much of a role in inhibiting the response time of the return current.

\subsubsection{Collisional Effects on Fast Particles}

\section{Stopping Power}

We have used the Spitzer ${ }^{[63]}$ and LMD $^{[64,65]}$ models to understand the collisions that take place non-relativistic temperatures. However, when electrons begin to travel at faster speeds, they are able to drive plasma oscillations and, also, must be treated relativistically. Work done by Atzeni ${ }^{[33]}$ and others, ${ }^{[68]}$ has given a model that describes the collisions of electrons in a plasma.

This model separates collisions into two components. The energy loss, or stopping power $\frac{d E}{d x}$ and the scattering $\frac{d \sigma^{2}}{d x}$. In this model, the stopping power changes the kinetic energy of the electrons and the scattering changes the directions of the particles' momentum.

The stopping power is given by the following equation, where $\rho$ is the mass density of the material, $\beta$ is the normalized speed of the electron, $m_{i}$ is the mass of the ions, $\hbar$ is the reduced Plank constant and $\gamma$ is the relativistic factor.

$$
\left.\frac{d \mathcal{E}}{d x}\right|_{\text {coll }}=-\rho \frac{1}{\beta^{2}} \frac{4 \pi q_{e}^{4} Z}{m_{i} m_{e} c^{2}}\left[\ln \frac{m_{e} c^{2}}{\hbar \omega_{p e}}+\frac{9}{16}-\frac{1}{2} \ln 2+f(\gamma)\right]
$$

where

$$
f(\gamma)=\ln (\beta \sqrt{\gamma-1})-\frac{\frac{1}{8}+\ln 2}{\gamma}+\frac{\frac{1}{16}+\frac{1}{2} \ln 2}{\gamma^{2}}
$$




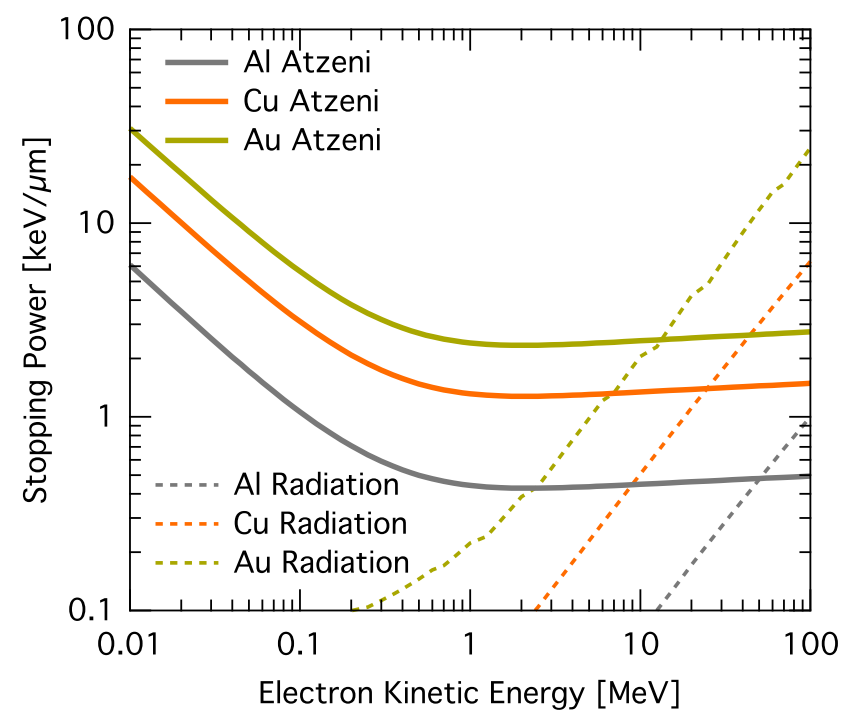

Figure 2.11: Energy loss, $\frac{d E}{d x}$, of electrons through solid density materials.

Unlike the Spitzer formula, we will only use this stopping power for individual electrons and not take an integration over a temperature. In both formulas there is a similar trend with both collisions models in that they both show a decrease in stopping with increased velocity. However, when electrons increase to a certain velocity then the stopping from collisions alone is no longer the main contribution to the stopping power. Instead, radiative stopping becomes the dominant contribution to the stopping power. The stopping powers for different materials at solid density is shown in Figure 2.11.

\section{Scattering}

The scattering as described in the Atzeni model ${ }^{[33]}$ gives the average square deflection per unit path length. As the electrons undergo multiple scattering events they become gaussian distributed with an average square deviation, $\left\langle\Theta^{2}\right\rangle$, as expected from the central limit theorem. The length scale for the increase in the square deviation is given below. Here $\ln \Lambda=\frac{4 \pi \lambda_{D}}{\lambda_{d B}}, \mathbb{Z}_{i}$ atomic number and $Z$ is the ionization state of the atom.

$$
\frac{d\left\langle\Theta^{2}\right\rangle}{d x}=2 \frac{q_{e}^{4}}{4 \pi \epsilon_{0}^{2}} \frac{1}{p^{2} v^{2}}\left[L_{e i}+L_{e e}\right]
$$




$$
\begin{aligned}
L_{e i} & =\mathbb{Z}_{i} n_{i} \times \mathbb{Z}_{i}\left(\ln \Lambda-\frac{1-\beta^{2}}{2}\right) \\
L_{e e} & =Z n_{i} \times\left(\ln \Lambda-\frac{\ln [2(\gamma+3)]+1}{2}\right)
\end{aligned}
$$

The scattering terms for electrons off of ions $L_{e i}$ and off of electrons $L_{e e}$ are separated. As one can see that ions scattering terms scales with $\mathbb{Z}_{i}^{2}$ and the electron term like $Z$. As we will be dealing with mostly high $\mathbb{Z}_{i}$ materials, we see that scattering due to electrons is very small compared to the ions.
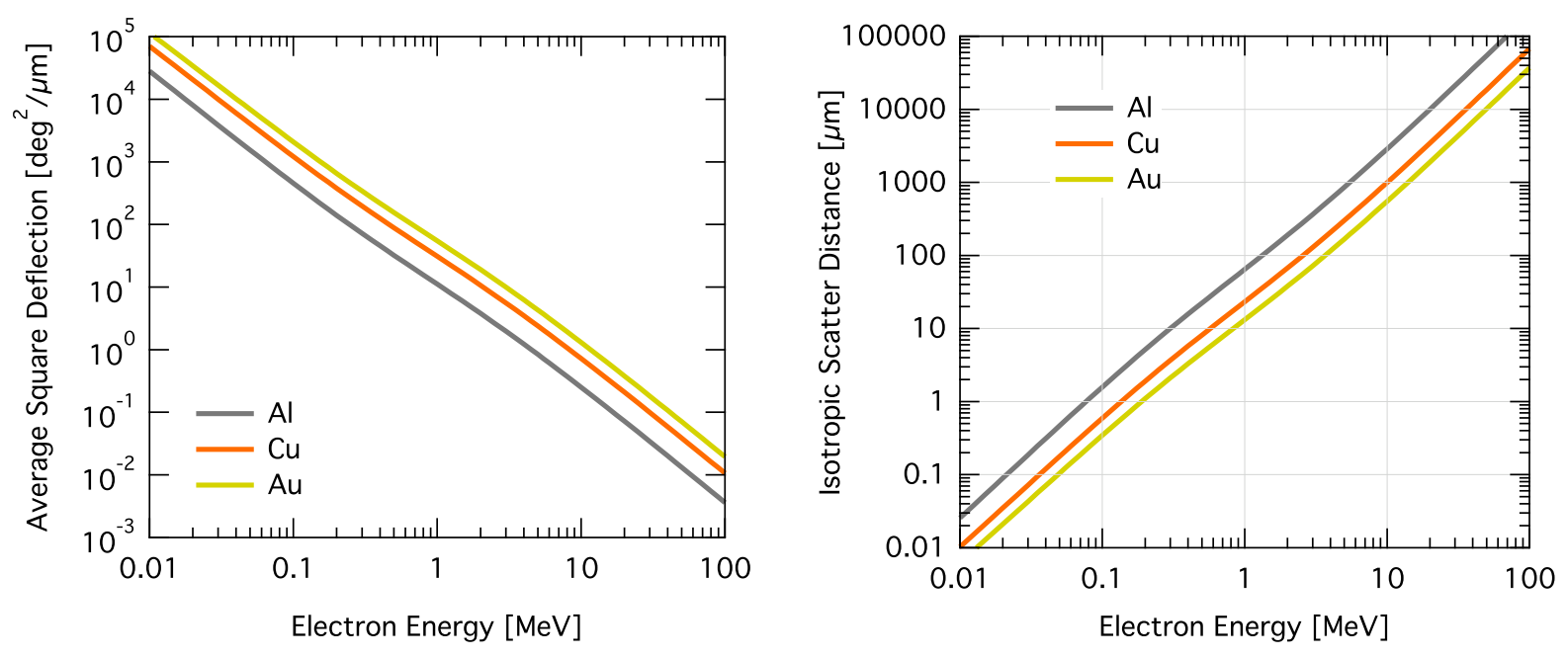

Figure 2.12: Average square deflection and Isotropic Scattering Distance for electrons in different materials at solid densities

Another intersting way to look at scattering is to determine the amount of distance that the electrons will have to cover before they are scattered isotropically into a sphere (i.e. $4 \pi$ ) if they began without any divergence. We call this the isotropic scattering distance. This is plotted in Figure 2.12 along with the average square deflection.

Notice that the isotropic scattering distance is only around 1-10 $\mu \mathrm{m}$ for electrons of 0.1 to $1 \mathrm{MeV}$ in $\mathrm{Cu}$ and $\mathrm{Au}$. Often the measurements that are of interest in our experiments are on the order of 100 to $1000 \mu \mathrm{m}$. Since electrons may be scattered significantly at these distances, the amount of initial divergence of the electrons may be of only minimal importance. 


\section{Laser Systems and Diagnostics}

\subsection{Laser System Descriptions}

The two lasers used for the experiments in this dissertation are the Titan Laser at the Lawrence Livermore National Laboratory and the Trident Laser at the Los Alamos National Laboratory. Both lasers use the optical parametric chirped pulse amplification (OPCPA) technique and both are Nd:glass lasers with a 1.054 $\mu \mathrm{m}$ wavelength. This allows them to achieve short laser pulses on the order to 500 fs and maximum energies up to around $100 \mathrm{~J}$. This gives to powers in hundreds of TW and achieves peak intensities of up to $10^{20} \mathrm{~W} / \mathrm{cm}^{2}$.

The CPA technique was developed by Strickland and Mourou ${ }^{[1]}$ in order to dramatically increase the maximum power acheiveable by lasers. The maximum power of a laser is restricted by the amount of laser intensity that the amplification optics can withstand without damage. One way to reduce the intensity on the amplifiers is to increase the diameters of these optics. However, this increases the cost of the amplifiers and manufacturing difficulties make this unfeasible. Another way to reduce the intensity is to increase the length of the pulse as it passes through the amplifiers; this is the method used in the CPA technique.This technique uses gratings (or other dispersive optics) to separate the laser by frequency. These different frequencies then travel different path lengths to spread out the pulse in time. This process is called chirping, and means that higher wavelenths proceed earlier in time that lower wavelengths (or vice versa). Since the pulse is longer its intensity is reduced while passing through the amplifiers and more energy can be added to the beam without exceeding the damage threshold. Then, after amplification, the pulse is recompressed by another grating which inverts the chirp and returns the 
laser to its original pulse length. Now the high energy beam is also high power and can achieve high intensities on target.

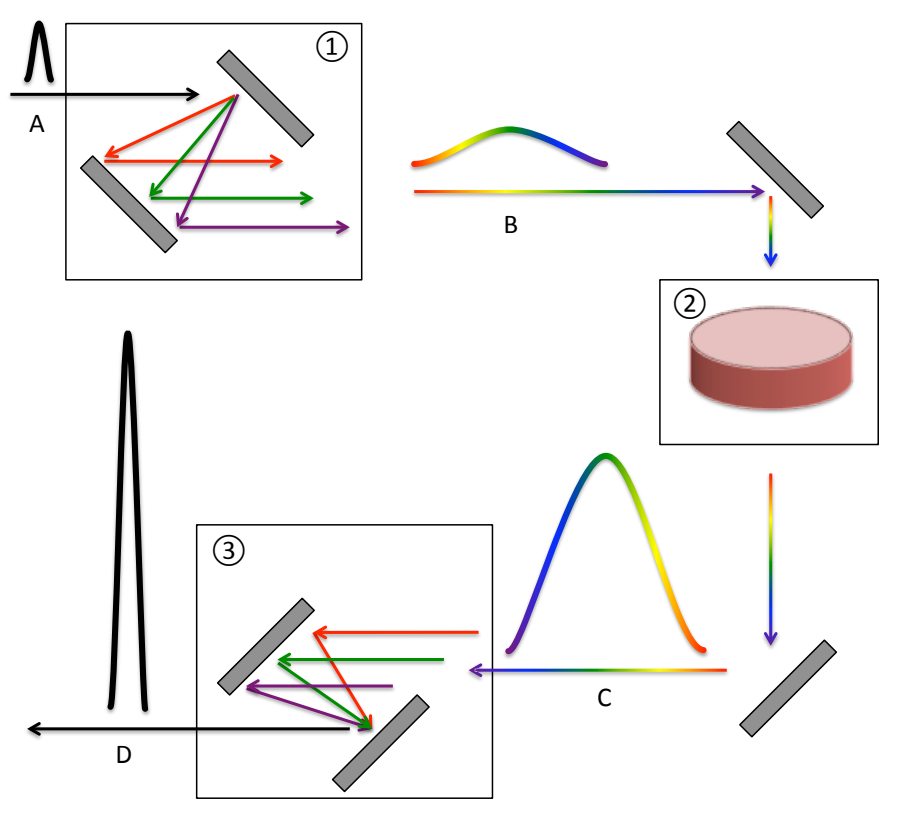

Figure 3.1: Illustration of the CPA amplification technique. The short unamplified pulse, A, enters a stretcher (1) where the pulse is chirped by wavelength to a longer pulse length, B. This pulse enters the amplification train (2) and gains energy. This chirped, amplified pulse, C, then enters a compressor (3), which produces the amplified, high-power, short laser pulse, D.

\subsubsection{Laser Power}

In terms of power delivered, the Trident and Titan laser systems are comparable, as shown in Table 3.1. This table shows the minimum and maximum laser energies used in this work. The power was varied on both laser systems to sample an overlapping intensity range.

\subsubsection{Laser Focus}

To understand how well the laser was focused at the target, a camera was placed at the laser focus and a low energy laser beam was captured by the camera. 
Table 3.1: Range of laser characteristics used in our experiments on the Trident and Titan laser systems. Also shown is the expected peak $\mathrm{T}_{\text {pond }}$ from the ponderomotive potential in (2.65), where the laser wavelength of both lasers is $1.054 \mu \mathrm{m}$.

\begin{tabular}{ccccccc} 
Laser & Range & $\begin{array}{c}\text { Energy } \\
{[\mathrm{J}]}\end{array}$ & $\begin{array}{c}\tau_{\text {FWHM }} \\
{[\mathrm{fs}]}\end{array}$ & $\begin{array}{c}\text { Peak Power } \\
{[\mathrm{TW}]}\end{array}$ & $\begin{array}{c}\text { Peak Intensity } \\
{\left[\mathrm{W} / \mathrm{cm}^{2}\right]}\end{array}$ & $\begin{array}{c}\text { Peak } \mathrm{T}_{\text {pond }} \\
{[\mathrm{MeV}]}\end{array}$ \\
\hline \multirow{2}{*}{ Trident } & Min & 24 & 560 & 40 & $1.8 \times 10^{19}$ & 1.5 \\
& Max & 75 & 570 & 122 & $5.3 \times 10^{19}$ & 2.9 \\
\hline \multirow{2}{*}{ Titan } & Min & 16 & 650 & 24 & $1.0 \times 10^{19}$ & 1.0 \\
& Max & 161 & 650 & 243 & $1.0 \times 10^{20}$ & 4.2
\end{tabular}

The camera had a high dynamic-range and was thus able to identify the peak of the laser in space as well as the lower intensity wings. Images of the laser profiles for the Trident and Titan beams are shown in Figure 3.2.

The diffraction limit of a laser is the smallest focal spot that is theoretically achievable for given laser setup. This is dependent only on the wavelength and the f-number of the laser. Both the Trident and Titan lasers have the same laser wavelength of $\lambda_{L}=1.054 \mu \mathrm{m}$. The f-number of parabola used on the Trident was $\mathrm{f} / 8$ and on Titan was $\mathrm{f} / 3$. Thus, all other things begin equal, one would expect that the Titan laser would focus to a smaller spot than Trident. However, as we will see shortly, this was not the case, due to beam aberrations on Titan that degraded the focal spot. On the other hand, we notice that the Trident beam is much closer to diffraction limited as can be seen from the Airy rings in the focal spot image of Figure 3.2. A lineout of the Trident focal spot is compared with its idea diffraction limited spot in Figure 3.3.

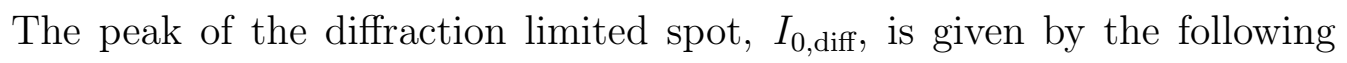
equation, dependent on the laser wavelength $\lambda_{L}$, the peak power $P_{0}$ and the fnumber $f_{L}$ of the focusing optic.

$$
I_{0, \text { diff }}=\frac{\pi}{4} \frac{P_{0}}{\lambda_{L}^{2} f_{L}^{2}}
$$




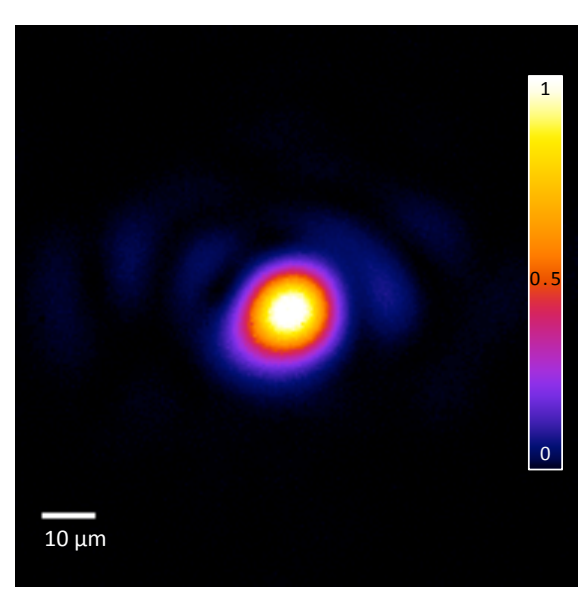

(a) Trident laser focal spot image.

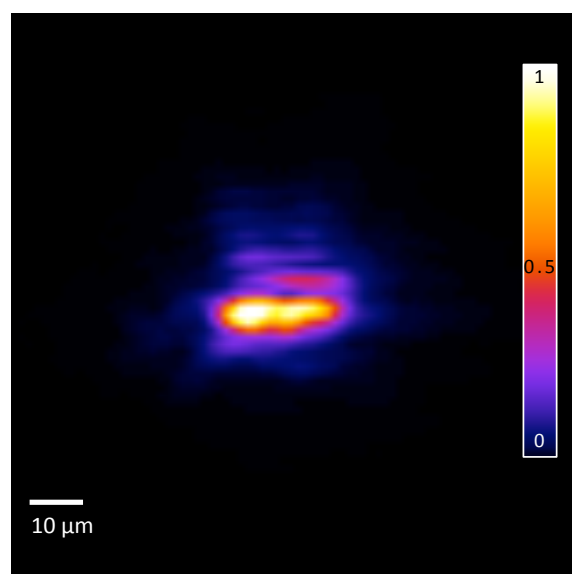

(b) Titan laser focal spot image.

Figure 3.2: Images of the two laser focal spots taken with high resolution cameras. The colorscale is linear with the maximum value normalized to the peak of the beams.

Also, the FWHM of the beam can be given by the approximation:

$$
\mathrm{FWHM} \approx 0.989 \lambda_{L} f_{L}
$$

Table 3.2: Comparison of the Trident and Titan focal spots versus diffraction limited beams.

\begin{tabular}{cccccrll} 
& & & Observed & Diffraction & Obs & $:$ & Diff \\
\hline Trident $(\mathrm{f}=8)$ & $\frac{I_{0}}{P_{0}}$ & {$\left[1 / \mathrm{cm}^{2}\right]$} & $4.12 \times 10^{5}$ & $1.10 \times 10^{6}$ & 2.68 & $:$ & 1 \\
& FWHM & {$[\mu \mathrm{m}]$} & 11.96 & 8.34 & 1 & $:$ & 1.43 \\
\hline \multirow{2}{*}{ Titan $(\mathrm{f}=3)$} & $\frac{I_{0}}{P_{0}}$ & {$\left[1 / \mathrm{cm}^{2}\right]$} & $5.11 \times 10^{5}$ & $7.86 \times 10^{6}$ & 15.36 & $:$ & 1 \\
& FWHM & {$[\mu \mathrm{m}]$} & 11.96 & 3.13 & 1 & $:$ & 3.82
\end{tabular}

To determine the quality of a given laser system, this diffraction limited

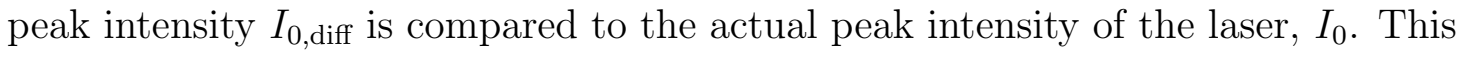
ratio is called the Strehl ratio $=I_{0} / I_{0, \text { diff. }}$ The Strehl ratio is 0.33 on the Trident laser and 0.065 on the Titan laser; thus the Trident laser focuses 5 times more effectively than the Titan laser. However, the Titan laser has a lower f-number 


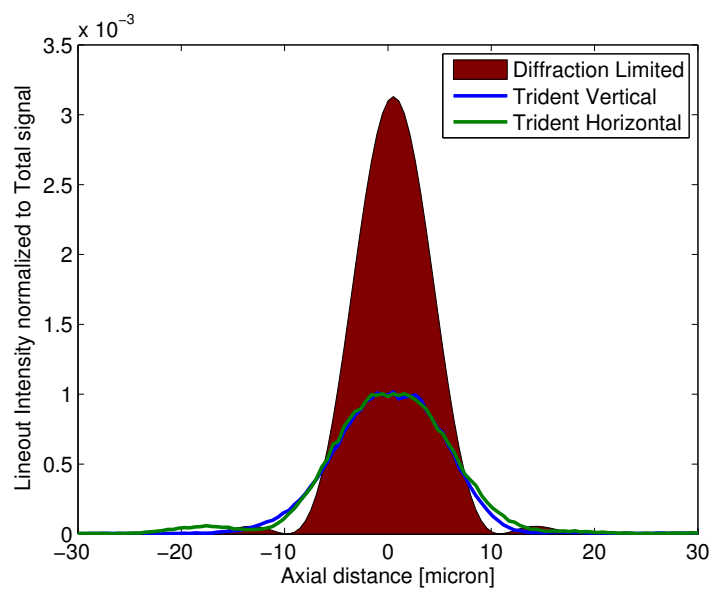

Figure 3.3: Plots of the diffraction limited spots (an Airy function) and the experimentally observed Trident setup.

than Trident, which cancels out the effect of this aberration and makes the focal spots of the two beams similar. This can be seen in similar focal sport of the two lasers shown in Figure 3.4, as well as the laser intensity distribution shown in Figure 3.5.

Table 3.3: Comparison of the Trident and Titan focal spots with respect to the energy contained in a region of the beam.

\begin{tabular}{c|cc|cc|} 
Energy Fraction & \multicolumn{2}{|c|}{ Trident } & \multicolumn{2}{c|}{ Titan } \\
& $\begin{array}{c}\text { Diameter } \\
{[\mu \mathrm{m}]}\end{array}$ & Peak Fraction & Diameter & Peak Fraction \\
{$[\mu \mathrm{m}]$} & \\
\hline $0 \%$ & $\mathrm{NA}$ & 1.00 & $\mathrm{NA}$ & 1.00 \\
$20 \%$ & 8.2 & 0.80 & 7.3 & 0.81 \\
$50 \%$ & 15.3 & 0.38 & 14.6 & 0.26
\end{tabular}

To get an understanding of how the intensity is distributed within the spot, an intensity distribution was taken. This is done by taking a histogram of the laser focal spot and determining how much energy (or power) has a given intensity. This is plotted for the minimum and maximum cases for Titan and Trident in Figure 3.5. In this figure, the intensity distribution for a triple gaussian fit to Trident has also been plotted. 


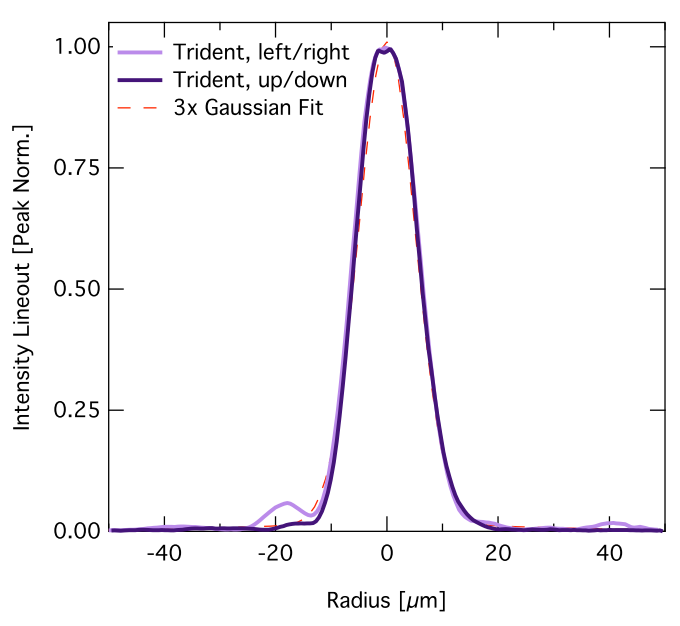

(a) Trident lineouts.

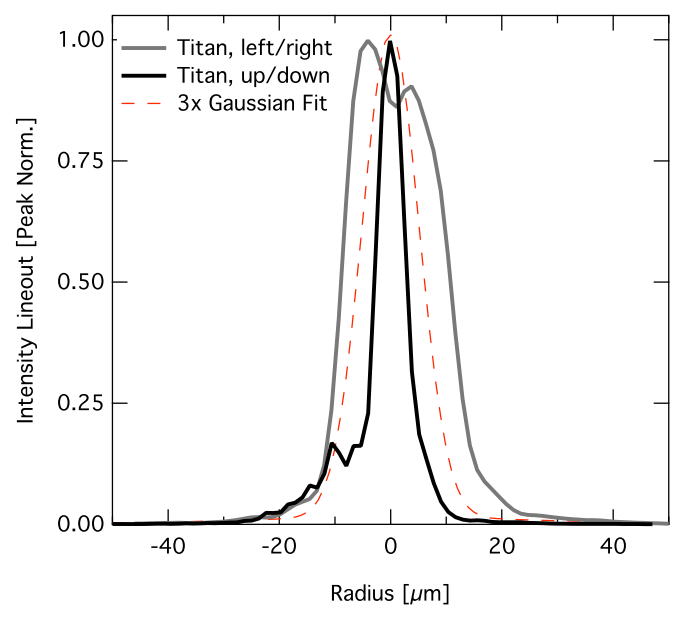

(b) Titan lineouts.

Figure 3.4: Lineouts of the Trident and Titan Intensity profiles in the vertical and horizontal directions in the solid lines. These are compared to a triple gaussian that was used in simulations.

Table 3.4: Triple gaussian function that fits the Trident focal spot and is also quite similar to the average of Titan. The peak intensity is the intensity when fit to the maximum energy Trident shot. The peak fraction is the normalized fraction of the peak. The FWHM is the full-width-at-half-maximum of the gaussian and the energy fraction is the fraction of energy the particular gaussian function contains with respect to the total.

First Second Third

$\begin{array}{ccccc}\text { Peak Intensity } & {\left[\mathrm{W} / \mathrm{cm}^{2}\right]} & 5.10 \times 10^{19} & 6.55 \times 10^{17} & 8.14 \times 10^{16} \\ \text { Peak Fraction } & & 98.58 \% & 1.266 \% & 0.1573 \% \\ \text { FWHM } & {[\mu \mathrm{m}]} & 11.96 & 58.63 & 108.7 \\ \text { Energy Fraction } & & 69.4 \% & 21.4 \% & 9.2 \%\end{array}$

A superposition of three 2-dimensional gaussian functions was fit to the laser profile of the Trident laser, as is shown in Figure 3.4. This function characterized the laser pulse and was useful in simulations of the laser. As noted previously, the Trident and Titan have somewhat similar laser profiles once they are averaged. 


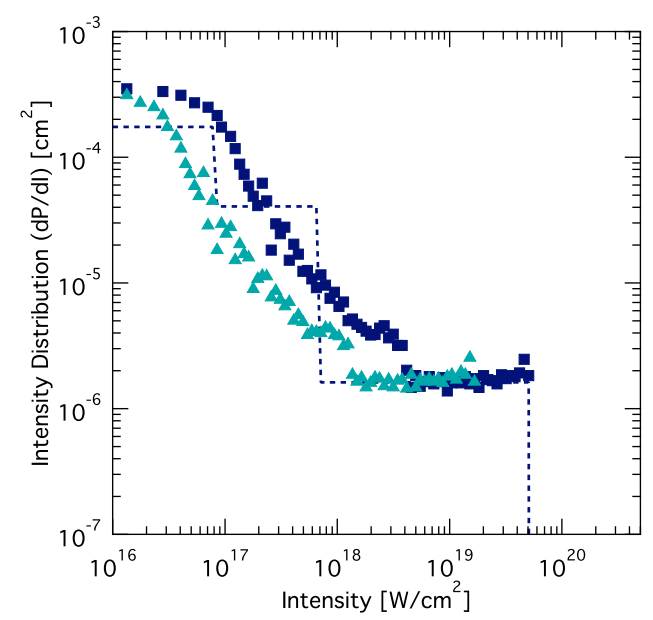

(a) Trident laser intensity profile for a $5 \times 10^{19}$ $\mathrm{W} / \mathrm{cm}^{2}$ (in squares) and a $2 \times 10^{19} \mathrm{~W} / \mathrm{cm}^{2}$ (in triangles) peak intensity laser pulse. The dotted line corresponds to the Gaussian fit for the highest intensity case.

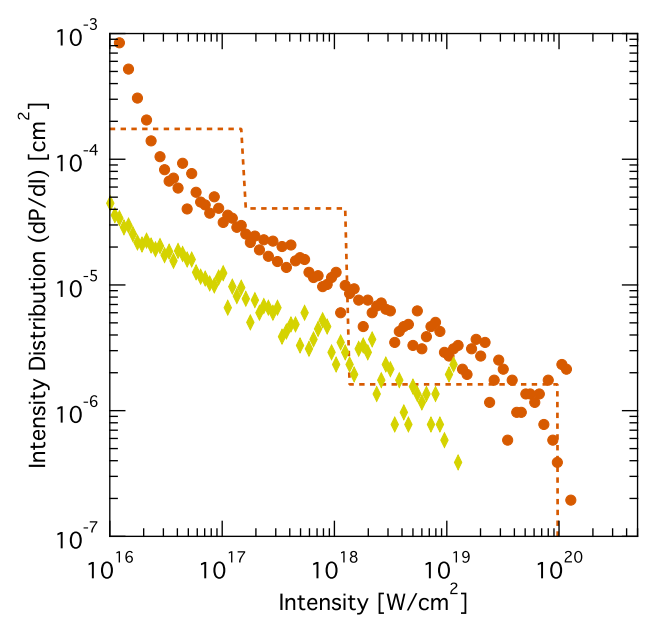

(b) Titan laser intensity profile for a $1 \times 10^{20}$ $\mathrm{W} / \mathrm{cm}^{2}$ (in circles) and a $1 \times 10^{19} \mathrm{~W} / \mathrm{cm}^{2}$ (in diamonds) peak intensity laser pulse. The dotted line corresponds to the Gaussian fit for the highest intensity case.

Figure 3.5: Intensity Distributions of the Titan and Trident lasers for the minimum and maximum powers as listed in Table 3.1. Shown with a dotted line is the triple gaussian fit to the maximum intenisty.

The intensity distribution of this triple gaussian function is shown in Figure 3.5 and the parameters of this function is shown in Table 3.4.

\subsubsection{Prepulse}

While the two lasers are similar, they differ in the amount of laser prepulse. Prepulse the energy in the laser that comes before the main pulse of the laser. Because of the extremely high peak laser intensities, even a prepulse that is one hundred million times less intense $\left(10^{-8}\right)$, like Titan, than the peak of the main pulse can have intensities up to $10^{12} \mathrm{~W} / \mathrm{cm}^{2}$, which is high enough to ionize and ablate material from the target. Since, in general, prepulses come nanoseconds before the main pulse, there is a significant amount of time for the initial target to become heated and to expand. This expanding plasma prior to the main pulse is called the preplasma. The preplasma moves the critical density of the laser 
further from the initial solid target. Also, importantly, it creates a large amount of underdense (below critical density) plasma, which may cause non-linear effects such as self-focusing of the laser to occur. The Titan laser has from $1 \mathrm{~mJ}$ (at lowest peak intensity) up to $18 \mathrm{~mJ}$ (at highest peak intensity) of prepulse energy that comes approximately 2 ns before the peak of the pulse. This creates preplasma that expands hundreds of microns in front of the initial target surface.
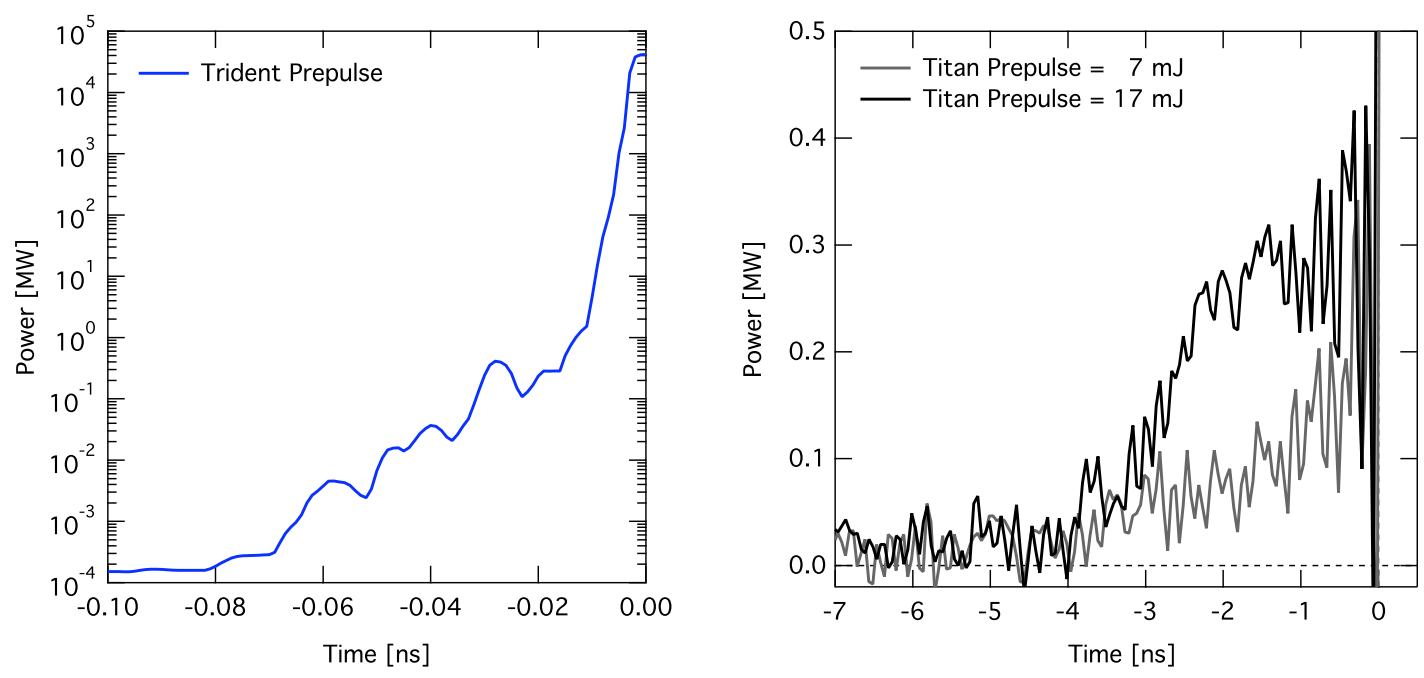

Figure 3.6: Prepulse levels of the Trident (left) and Titan (right) Laser systems.The Trident level of prepulse, plotted on a log-scale) replotted from Ref. [18, is much lower than on Titan, plotted on a linear scale.

The Trident laser has a significantly reduced prepulse level, while still having a high laser power. ${ }^{[69]}$ In fact, the amount of energy coming nanoseconds before the main pulse is less than $1 \mu \mathrm{J}$. Therefore the main contribution to preplasma creation is not from laser energy coming nanoseconds before the main pulse. Instead, it comes from the finite rise time of the laser that comes around $0.1 \mathrm{~ns}$ before the main pulse, as shown in Figure 3.6. In this relatively short amount of time, the Trident laser may deliver around $5 \mathrm{~mJ}$ of energy, which is similar to the energy in Titan's prepulse. However, as this material has much less time to expand away from the target, there will be much less preplasma. As shown previosly, and repeated below, the scale length of the ablated plasma is dependent on both the 
plasma temperature and the expansion time.

$$
\text { Expansion Length } \propto C_{s} t=t \sqrt{\frac{Z k_{B} T}{m_{i}}}
$$

This difference in preplasma scale length between Trident and Titan is the primary reason for differences in the high-intensity laser-matter interactions between the two lasers.

\subsection{Overview of $\mathrm{K} \alpha$ Radiation}

The main technique used to diagnose the electron spectra in this dissertation is the measurement of $\mathrm{K} \alpha$ radiation emitted by copper. These x-rays give a way to measure the electrons passing through a material. They are generated when an electron from the L-shell falls into the K-shell and the excess energy is released as a photon, called a $\mathrm{K} \alpha$ photon. This process is shown in Figure 3.7. In copper, this photon has an energy around $8 \mathrm{keV}$.

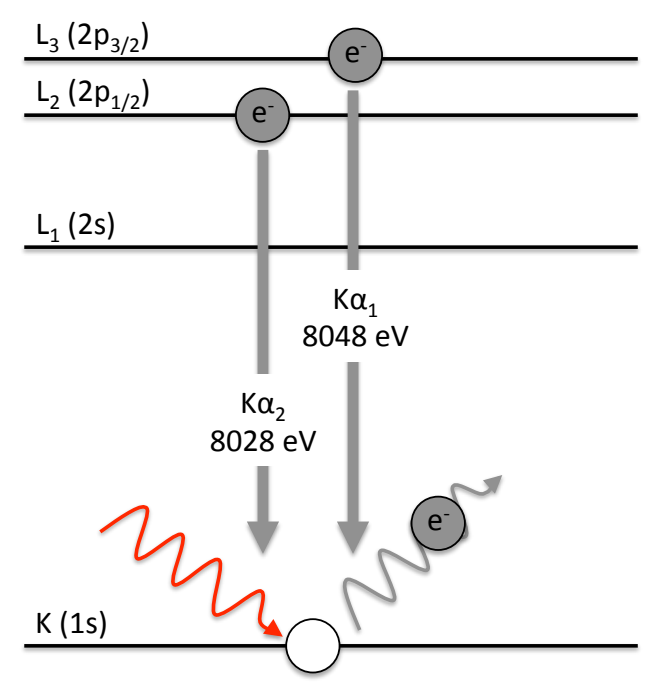

Figure 3.7: Illustration of the generation of $K_{\alpha}$ radiation. Ionizing radiation (e.g. a fast electron) ionizes a k-shell electron, which causes the k-shell vacancy. When an electron from the L-shell drops to fill this vacancy it releases a photon with an energy equivalent to the difference in energy of the two states. This is called a $\mathrm{K}_{\alpha}$ photon. 
The $\mathrm{K} \alpha$ generation process occurs when the $\mathrm{k}$-shell is vacated. The shell can be vacated when ionizing radiation impacts the k-shell electron and imparts energy to overcome the binding energy. In our experiments, the main process that ionizes the atoms are the collisions with fast electrons moving through the material. Figure 3.8 shows the cross-section for k-shell ionization by electrons in copper. Notice that the cross-section is relatively flat for energies between 0.5 to $10 \mathrm{MeV}$. This means that the $\mathrm{K} \alpha$ radiation can be thought of a way to count electrons that pass through a plane of the material.

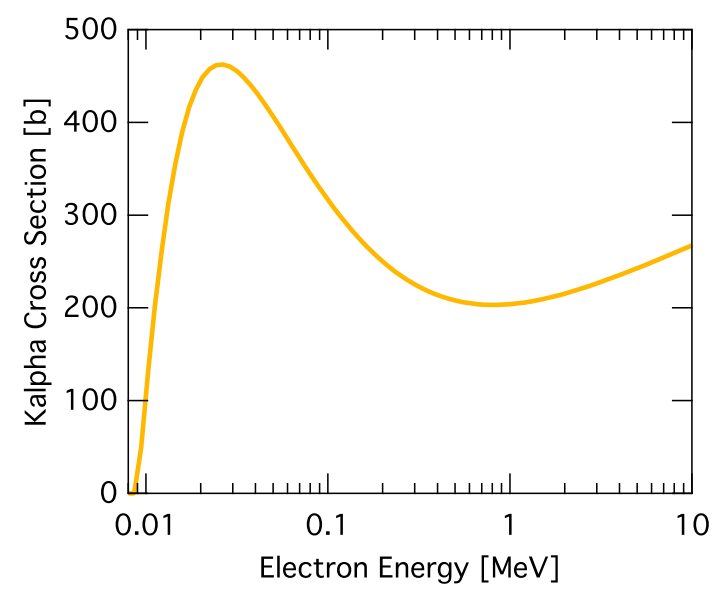

Figure 3.8: Cross-section for k-shell ionization in copper due to fast electrons. From equations in Refs. ${ }^{[70,71]}$

\subsection{Crystal Spectrometer for $\mathrm{K} \alpha$ Measurement}

\subsubsection{Bragg Reflection and Spectrometer Information}

To characterize the total energy carried by electrons we measured the total $\mathrm{K}_{\alpha}$ radiation generated from the target using an absolutely calibrated spectrometer. This spectrometer uses Bragg reflection from a crystalline material to separate the different wavelengths. Bragg reflection occurs as x-rays passing through a material cause the atoms within the material to oscillate. If the atoms are aligned in layers then the oscillations can be in phase. This phase matching criterion is 
met when photons of a given wavelength are incident at the appropriate angle, known as the Bragg angle. Then the reradiation of the atoms will align coherently and produce a specular reflection. This can be understood through the graphic in Figure 3.9. Here the wave $B$ traverses an additional length of $2 d \sin \theta$. If this distance is equal to an integer multiple $n$ of the wavelength $\lambda$, then the waves will be in phase. This leads to the Bragg relation of $n \lambda=2 d \sin \theta$. Thus different $\mathrm{x}$-ray wavelengths will have different reflection angles and a crystal can be used as a spectrometer.

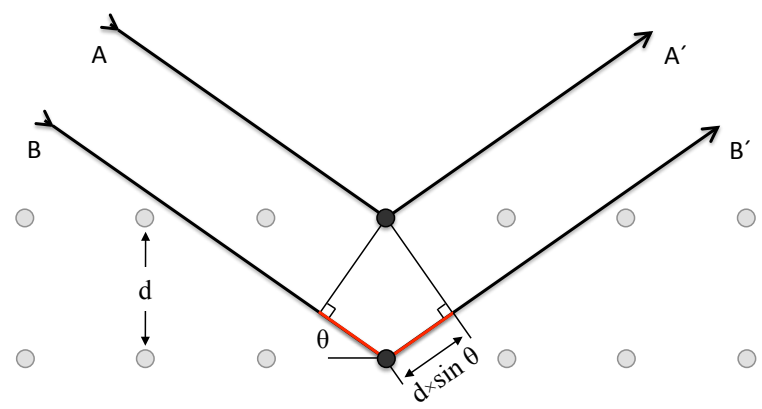

Figure 3.9: Illustration of Bragg reflection in a crystal. Incoming light rays $A$ and $B$ are reflected if they stay in phase. They rays will stay in phase if they traverse the same distance plus an integer, $n$, number of wavelenghts $\lambda$. If this condition is not met the light wave will not be in phase and thus not propagate.

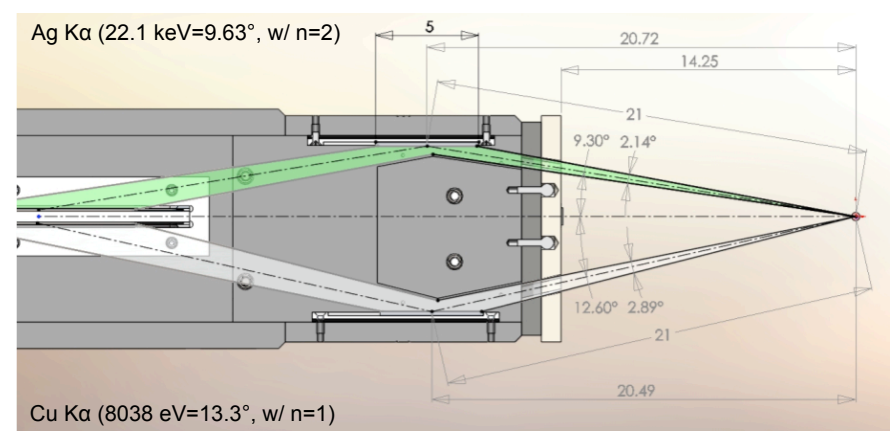

Figure 3.10: Dimensions of the HOPG spectrometer. The shaded green line on top corresponds to a portion of the spectrometer use to diagnose $\mathrm{Ag} \mathrm{K}_{\alpha}$ radiation, which was not used in this work. The bottom portion in shaded gray corresponds to the $\mathrm{Cu} \mathrm{K}$ 解 was used. Image courtesy of K. Akli 
In our experiments, we use highly oriented pyrolytic graphite (HOPG) which consists of many crystaline planes that are oriented within some deviation from normal. By allowing some deviation from a perfect crystal the reflectivity is increased since more x-rays match the Bragg condition at a given point. However, for this same reason, the resolution of the spectrometer is degraded. For measuring the absolute value of $\mathrm{K}_{\alpha}$ radiation, the resolution of the HOPG is acceptable and the high reflectity gives high signal-to-noise ratios. The dimensions of the HOPG spectrometer used in our experiments is shown in Figure 3.10.
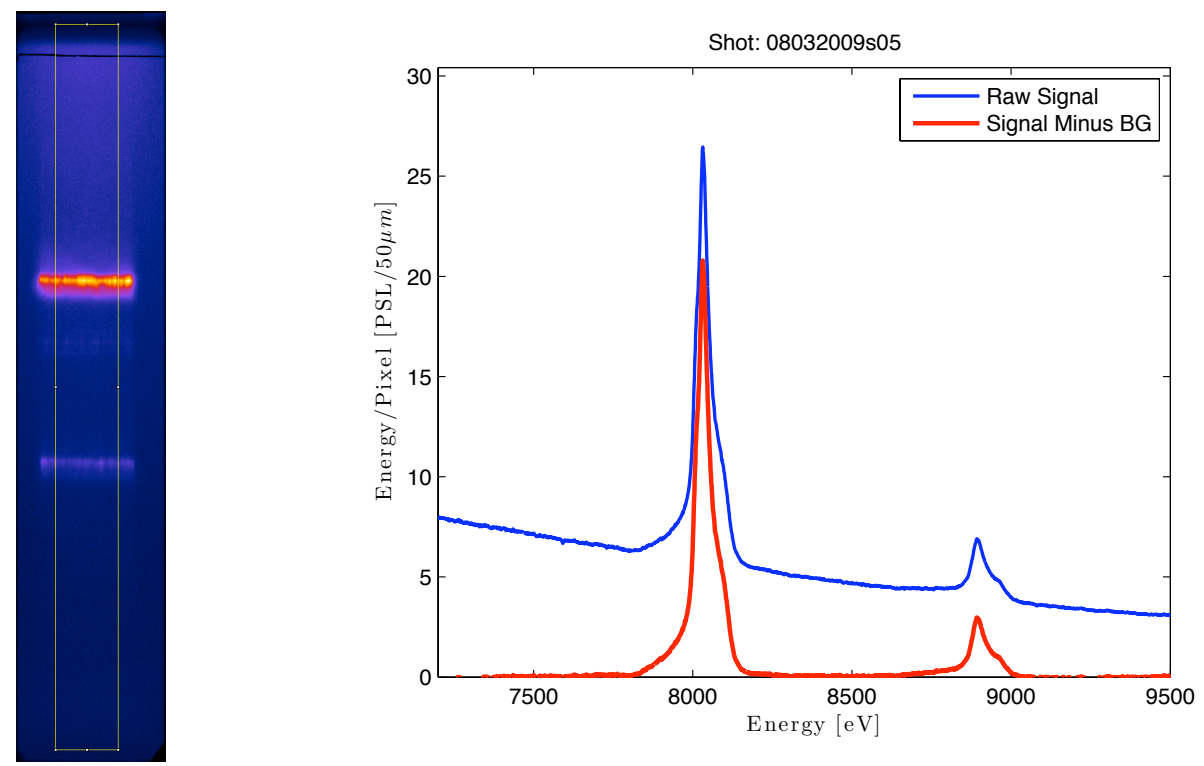

Figure 3.11: (Left) Raw pseudo-color image of an imaging plate after exposure within the HOPG spectrometer. (Right) Transversely averaged lineout of the HOPG imaging plate, before and after subtraction of the continuum background radiation.

A sample image of raw data from the HOPG spectrometer is shown in Figure 3.11a. To obtain a spectrum from this data a large box (in the yellow) was averaged transversely (horizontally) and then a lineout was taken in the energy direction (vertical) axis. This lineout is shown in Figure 3.11a. The $\mathrm{K}_{\alpha}$ line, as well as a $\mathrm{K}_{\beta}$ line are seen in the peaks. Notice the low level of noise in this lineout, this is due to the detailed construction of the diagnostic and the large amount of 
shielding used. After subtracting the continuum background, we integrate under the $K_{\alpha}$ peak to get the total $K_{\alpha}$ yield.

\subsubsection{Spectrometer Calibration with SHCCD}

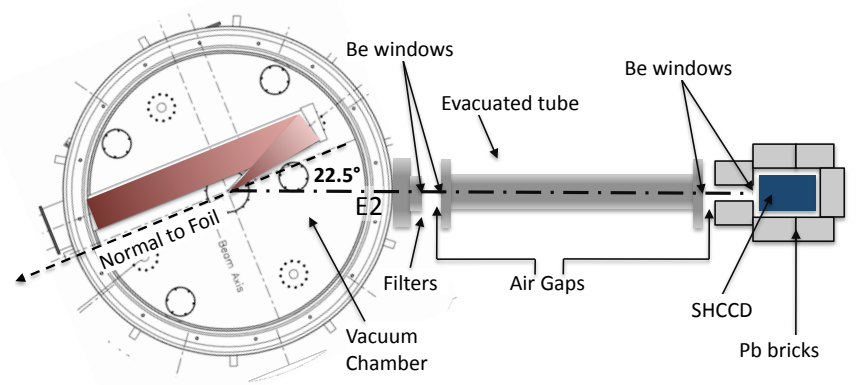

Figure 3.12: Chamber diagram of the cross-calibration setup for the SHCCD, not to scale.

To calibrate the spectrometer, shots were taken with the Titan laser incident on a $\mathrm{Al} / \mathrm{Cu}$ multi-layered foil, as shown in Figure 3.12. The $\mathrm{K}_{\alpha}$ emission was measured with the HOPG spectrometer and cross-calibrated against a previously calibrated $^{[72]}$ Single Hit CCD (SHCCD). The SHCCD records the energies of individual photons incident on a single pixel of the CCD. By taking a histogram of these photons a spectrum is recorded and the total number of $\mathrm{K}_{\alpha}$ photons is recorded. The amount of charge gathered by each pixel is linearly related to the energy of the x-ray photon incident on that pixel. We cannot differentiate between a single photon and multiple photons hitting a single pixel, thus it is important to operate in the so-called single hit regime, where the fluence of photons is low compared to the pixel size, so that multiple photons will not hit a single pixel.

A sample of a portion of the CCD is shown in Figure 3.13, which shows that the photon density is low compared to the size of the CCD. A single event algorithm was used to subtract pixels that may have been caused by overlapping photons. The energy resolution of the SHCCD is of much lower quality than the HOPG. In fact, the SHCCD cannot distinguish between the desired $\mathrm{K}_{\alpha}$ emission 

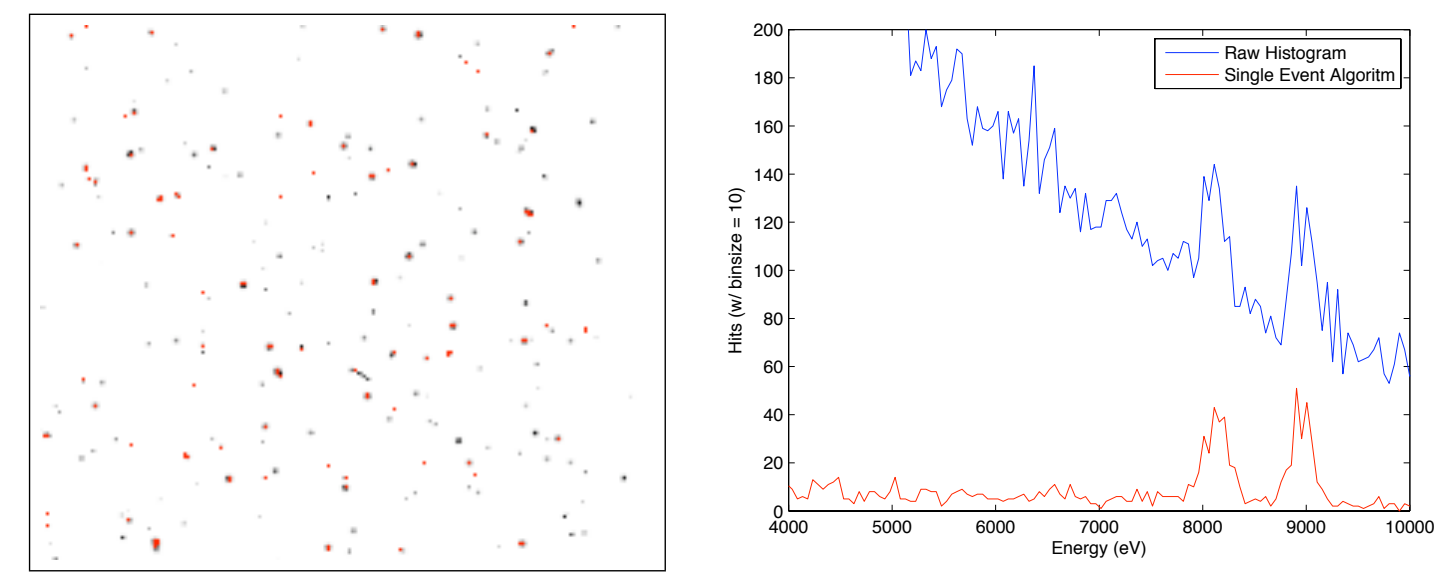

Figure 3.13: (left) Raw image from SHCCD. The black pixels have energies consistent with $\mathrm{K} \alpha$ photons, while white pixels contain less energy and red pixels contain more energy than consistent with K $\alpha$. (right) Spectra of energies contained on the SHCCD before the single event algorithm (to remove double hits) in blue and after in red.

and a thermally emitted $\mathrm{He}_{\alpha}$ line from $\mathrm{Cu}$. The $\mathrm{He}_{\alpha}$ line is excited when the laser is incident upon the $\mathrm{Cu}$, therefore the target was coated with $5 \mu \mathrm{m}$ of $\mathrm{Al}$ on the laser interaction side.

The cross-calibration done between the SHCCD and the HOPG spectrometers is shown in Figure 3.14. Since the SHCCD has been absolutely calibrated by Maddox et al., ${ }^{[72]}$ the HOPG can now be used to obtain quantitative data.

\section{4 $\mathrm{K} \alpha$ Spherical Imager}

A spherical imager was used to obtain an image of the $\mathrm{K} \alpha$ radiation emitted by the target. This crystal was quartz with a $50 \mathrm{~cm}$ radius of curvature a 0.3082 nm 2d-lattice spacing and 2131 Miller Indices. The imager uses Bragg reflection off a spherical mirror to create a focused x-ray image.

The focused light was captured by one of two collection instruments. The collector used on Titan was an x-ray sensitive charge-coupled device (CCD), PISX1300. This device counts the number of electrons freed by an x-ray photon 


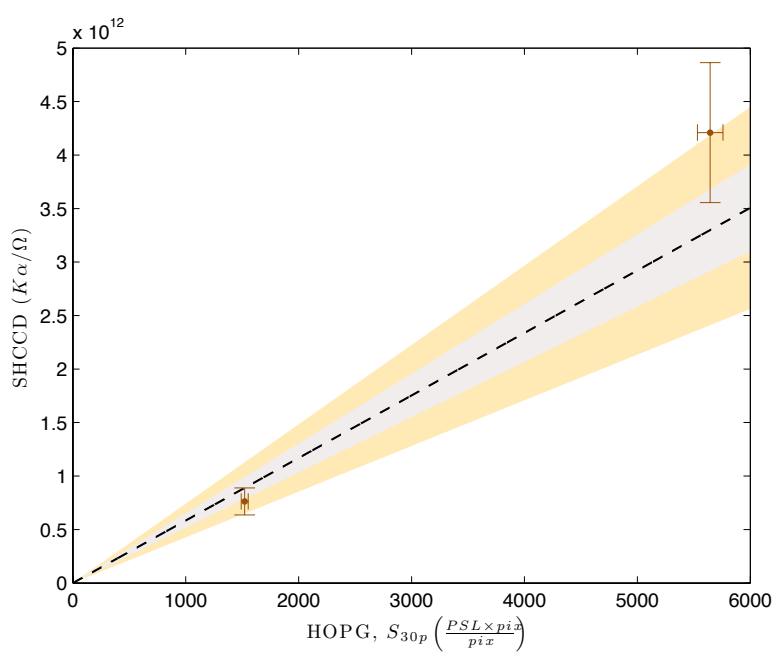

Figure 3.14: Calibration of the HOPG spectrometer against the SHCCD spectrometer. The dark orange dots are the experimental values with error bars. The dotted black line shows the calibration value. The shaded gray region corresponds to an error bar of $12 \%$, which is the value found from summation of the expected error bars from the calibration. The shaded orange region corresponds to an error of $27 \%$, which corresponds to the error expected from the deviation of these points from the average.

traveling through Si by running the freed electrons through a circuit. CCD detectors are extremely useful due to their high signal-to-noise ratio (SNR) and good resolution. However, due to their susceptibility to electromagnetic interference they cannot be used in all situations. In fact, a more robust technique was used on Trident. The collectors used on Trident were FujiFilm Imaging Plates (IP). The IPs contain a phosphor layer that is sensitive to ionizing radiation. When energetic particles (e.g. electrons, photons, ions) are incident on this phosphor they excite electrons. Some of these electrons do not fall back immediately into their ground state and instead are trapped within phosphorescent material. These electrons can be freed from this trapped state by illumination with a red laser $(\lambda \sim 600$ $\mathrm{nm})$. Upon decay back into the ground state these electrons emit another photon $(\lambda \sim 400 \mathrm{~nm}) .{ }^{[73]}$ By shining a red laser on the exposed IP and recording the reemitted light, an image of the absorbed radiation can be reconstructed. This is 
done within a FujiFilm scanner. There is some chance for the electrons trapped in the phosphor to spontaneously decay to the ground state. For this reason, it is important to scan with the laser at a fixed period after each exposure. Additionally, since $600 \mathrm{~nm}$ light is present in natural white light, it is important to keep the IPs light-tight before scanning to avoid pre-luminescence. Unfortunately, IPs have a lower signal-to-noise ratio (SNR) than CCDs, but their robustness to EM interference make them valuable in many situations.

Due to differences in detectors as well as different imager geometries the resolution of the image setups was different at each laser facility. On Trident the resolution was characterized by a $60 \mu \mathrm{m}$ FWHM gaussian smoothing function and a $40 \mu \mathrm{m}$ FWHM on Titan.

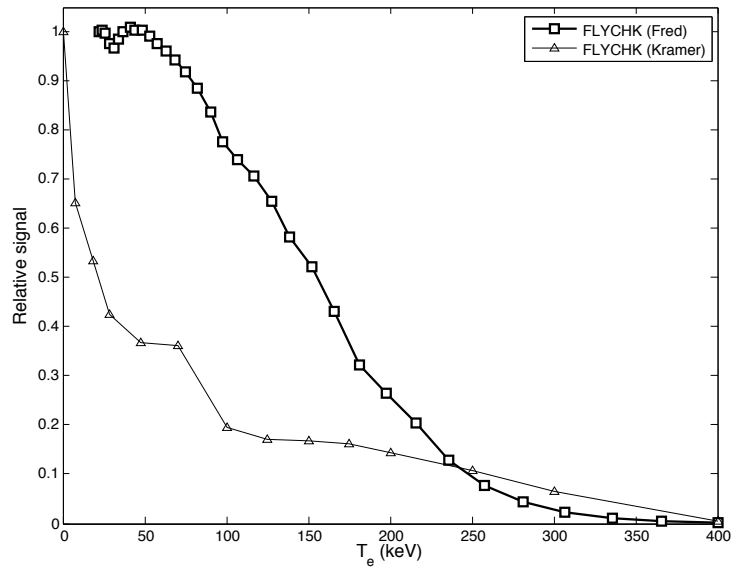

Figure 3.15: Shifting of the $K_{\alpha}$ image as a function of temperature. Image courtesy of F. Pérez.

The $\mathrm{K}_{\alpha}$ imager has a very low bandwidth of $6 \mathrm{eV}$ centered at $8048 \mathrm{eV}$. So if the $K_{\alpha}$ line shifts spectrally it causes the emission to be decreased. ${ }^{[53]}$ The recorded signal would be reduced in high temperature regions even if the same amount of $K_{\alpha}$ was emitted. This phenomenon includes both actual shifting cause by ionization for instance and broadening, caused by Stark or doppler broadening. All effects caused by an increase in the Copper temperature. However, we have run simulations using atomic codes that show these processes should not play much of an role until temperatures reach above $100 \mathrm{eV}$ as is shown in Figure 3.15. All of 
our modeling suggests that such temperatures are not reached our experiments. Additionally, to verify that such shifting/broadening did not occur we looked at the ratio of integrated yield from both the $\mathrm{K}_{\alpha}$ imager and the HOPG spectrometer and verified that their ratio was constant for a given experimental setup over the range of intensities studied. If there was significant shifting/broadening we would expect to see variation in this ratio as a function of laser energy, which was not observed, as shown in Figure 3.16.

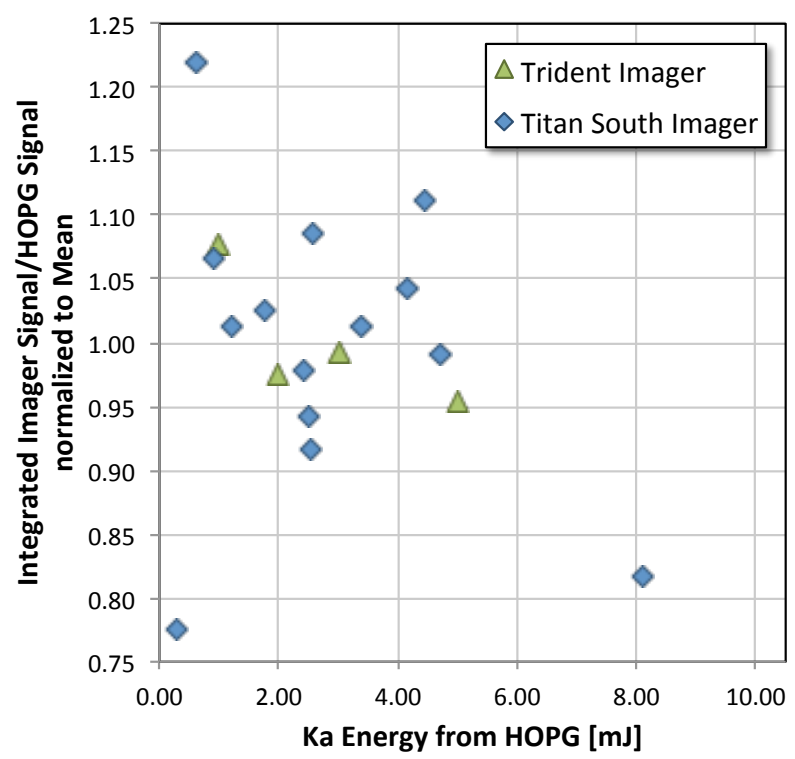

Figure 3.16: Ratio of integrated $\mathrm{K} \alpha$ signal from the HOPG spectrometer versus the integrated signal from the $\mathrm{K} \alpha$ imager. No dependence on $\mathrm{K} \alpha$ signal level is observed and the ratios are fairly consistent, which is in agreement with the assumption that no shifting was observed. 


\section{Particle-In-Cell Simulations}

\subsection{The direct-implicit PIC Method}

The interaction of high-intensity lasers with solid-density matter creates a significant challenge for computer simulations. On one hand, individual electron motion must be resolved to capture the non-linear interaction of these relativistic particles with the laser. On the other hand, the number electrons within a relatively small $10 \mu \mathrm{m}$ cube is already around $10^{14}$ which is a tremendously large number to simulate. Fortunately, the development of particle-in-cell (PIC) simulations has been developed to meet this challenge. In our simulations we will use the direct-implicit PIC code LSP. ${ }^{[55]}$

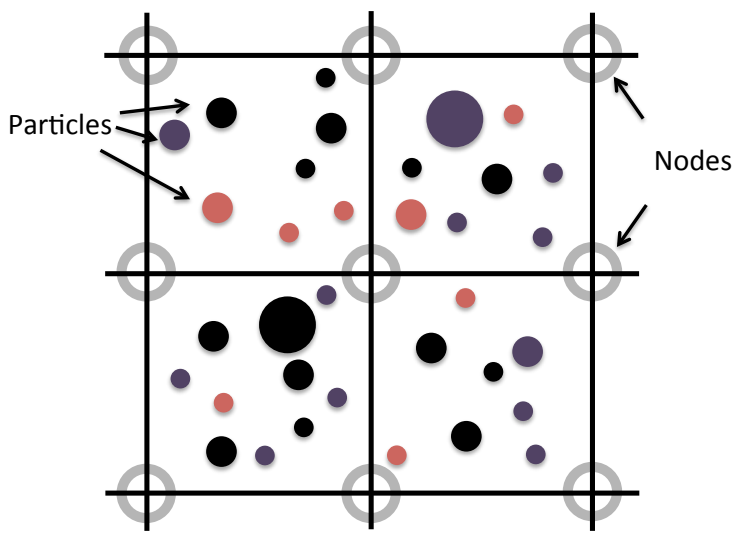

Figure 4.1: Cartoon of a PIC simulation with macro-particles existing continuously in space and the fields calculated on a spatial grid.

The PIC approach to these problems is to aggregate the mass and charge of many similar individual particles into one entity; called a macro-particle. In LSP, 
the macro-particles exist continuous in the space, while fields are calculated along a grid imposed in space as shown in Figure 4.1. The macro-particles preserve the charge-to-mass ratio of the individual particles and thus react identically to the Lorentz force that drives laser-acceleration. The acceleration due to this force is presented in discrete form ${ }^{[55]}$ in (4.1).

$$
\frac{\Delta \widehat{\mathbf{p}}}{\Delta t}=\frac{q}{m c}\left[\mathbf{E}+\frac{\widehat{\mathbf{p}}}{\gamma} \times \mathbf{B}\right]
$$

Here $q, m$ and $\widehat{\mathbf{p}}$ are the charge, mass and normalized momentum $(\widehat{\mathbf{p}}=$ $\mathbf{p} / m c$ ) of the particle. The speed of light is $c, \Delta$ denotes changes over a time step, and $\mathbf{E}$ and $\mathbf{B}$ are the electric and magnetic (EM) fields. The motion of these particles then influences the development of EM fields through the discrete Maxwell's curl equations ${ }^{[55]}$ shown in (4.2) and (4.3), where $\mathbf{J}$ is the current and $\widetilde{\mathbf{S}}$ is the implicit susceptibility tensor, to be explained shortly.

$$
\begin{aligned}
& \frac{\partial \mathbf{E}}{\partial t}=\nabla \times \mathbf{B}-\mathbf{J}-\widetilde{\mathbf{S}} \cdot \mathbf{E} \\
& \frac{\partial \mathbf{B}}{\partial t}=-\nabla \times \mathbf{E}
\end{aligned}
$$

In the so-called explicit PIC model, the EM fields are calculated at exactly the same time as they are used to accelerate particles. In order for this method to maintain numerical stability the plasma frequency must be resolved at all times and locations (i.e. $\omega_{p e} \Delta t<1$ ). However, for solid-density material, resolving such a time scale is not computationally feasible. To address this difficultly, the so-called direct-implicit method was developed. ${ }^{[74]}$ This is used by LSP in the following way: Instead of calculating the Lorentz force from quantities known at the present time, it uses a predictive method to estimate what the EM fields and momentum will be in the future and averages this with the past. To correct for errors created by these predictions a corrective implicit susceptibility tensor $\widetilde{\mathbf{S}}$ added to the electric field evolution.

This predictor-corrector method has the effect of smoothing over highfrequency modes. This allows the implicit simulations to remain stable even when 
the plasma frequency $\omega_{p e}$ is unresolved, though in such situations plasma waves will also be unresolved. Thus this method does not exactly allow the modeling of plasma physics within solid-density materials, but it does allow plasma physics to be modeled alongside such materials. For instance, in this dissertation, a laser accelerates electrons from a hot low-density plasma into a cold solid-density material. Such a situation ideal for an implicit PIC model. The model resolves $\omega_{p e}$ for the laser interaction in the plasma, and can computationally-manage solid density material, where resolution of $\omega_{p e}$ can be neglected due to strong collisional effects.

Another feature in LSP that provides increased stability and accuracy at decreased computational cost is its fluid/kinetic approach to particles. In LSP, a kinetic macro-particle is considered as a single particle, as described above. On the other hand, a fluid particle is a representative of a non-relativisitc Maxwellian distributed ensemble of particles that have both a temperature and a directed energy. In addition to being pushed by the Lorentz force, such particles are also explicitly influenced by the pressure and temperature gradients created by adjacent fluid particles. As these particles already represent a full distribution function, such a distribution need not be resolved discretely with individual particles. Thus this fluid modeling allows for a decreased number of particles, which reduces the computational load. In general, these fluid particles are used to model the background high-density material; since they are non-relativistic they cannot be used where the laser is present.

\subsection{Collisions and Resistivity}

As discussed above, the simulations in this dissertation often include soliddensity materials, where collisional effects dominate over the plasma response. In LSP the collisions are treated with two separate methods. The high-energy relativistic particles use a test-particle model. ${ }^{[33]}$ While the background collisions between use a grid-based method with an averaged collision frequency. ${ }^{[75]}$

The collisions within the background material are important to accurately model the return current within the plasma. This return current is generated to 
satisfy the current neutrality requirement as we have described in Section 2.4.3. This return current is important because it is low energy and thus is very collisional. As the electrons collide within the material, they induce an electric field. This can be thought of as an Ohmic field, with $E=\eta j$, where $E$ is the electric field, $\eta$ is the material resistivity and $j$ is the current density of the return current. In some, so-called hybrid-PIC codes $^{[76,77]}$ the resistivity is imposed as a value taken from the properties of the materials. However, in LSP the resistivity arrises from modeling of the particles collisions themselves, which then gives an effective resistivity. The collision frequencies for fluid particles used in LSP are taken from the Lee-More ${ }^{[65]}$ model with Desjarlais ${ }^{[65]}$ corrections, called the LMD model. This model is dependent on the ion density, ionization state and electron temperature of the material. This assumes that the plasma is locally charge neutral, which is not the case when plasma waves are included, as when modeling the laser interaction. In such situations, the background material is represented by kinetic particles that undergo collisions using the Spitzer model, ${ }^{[63]}$ which is capped at the peak collision-frequency found from LMD, usually around $100 \mathrm{eV}$.

\subsection{Benchmarking}

The collisions of the high-energy relativistic particles are modeled using a test-particle method. ${ }^{[33]}$ In the high-density material these collisions will play a major role in both the stopping power of electrons and the amount of angular deviation that they experience.

Before using LSP to model our experiments, it was necessary to verify that LSP was implementing the physical models in an accurate way. The experiments that will be modeled rely heavily on the transport of electrons through matter and the $\mathrm{K}_{\alpha}$ radiation that they generate. Thus it was important to verify the stopping and scattering of LSP, as well as, the $\mathrm{K}_{\alpha}$ generation. 


\subsubsection{Benchmarking of Stopping Power}

In LSP the collisions of relativistic electrons is split into two components: the stopping power, $\frac{d E}{d x}$, and the scattering, $\frac{d \sigma^{2}}{d x}$. The stopping power modifies the energy of the electrons (i.e. the total momentum of the electron) and the scattering modifies the direction of the direction of the particles momentum. LSP uses what is know as the Atzeni ${ }^{[33]}$ as discussed in Section 2.4.5

This stopping power takes into account both the energy lost from the relativistic electrons colliding with the background plasma and the energy that is lost to plasma waves in the background plasma. Due to the fact that LSP will not be resolving the plasma waves in our solid density targets, this is the appropriate equation to use.

To correctly benchmark the stopping power, we must remove factors that would change a particles energy. So in this simulation we completely turn off the fields. Naively, one might think that the measuring the distance that an electron traveled before stopping would be sufficient to verify that the stopping power was correct. However since the electrons also scattering in the material, this effectively shortens the distance that they travel, which makes it a poor way to verify the stopping power. So instead the following process was used: A mono-energetic beam of electrons was injected at the same instance in time into an infinite block of solid $\mathrm{Cu}$. As the electrons all began with the same energy at the same time, the total energy of the beam equal to the number of electrons multiplied by the energy of each electron, $\mathcal{E}$. Since the electrons are losing energy at the same rate, the energy loss per time, $\frac{d \mathcal{E}}{d t}$, is determined.

$$
\frac{d \mathcal{E}}{d t}=\frac{\Delta \mathcal{E}}{\Delta t} \frac{d t}{d x}=\frac{\Delta \mathcal{E}}{\Delta t} \frac{1}{v}=\frac{\Delta \mathcal{E}}{\Delta t} \frac{1}{c \sqrt{1-\left(\mathcal{E} / m c^{2}+1\right)^{-2}}}
$$

Thus, with this method, it is possible to determine the stopping power that LSP is using without the complications of scattering.

In Figure 4.2 the results of the LSP simulation and analysis are plotted against the analytically derived Atzeni stopping power. ${ }^{[33]}$ Notice that they match quite well, however the Atzeni stopping model does not include the energy loss due to radiation, which is a major loss of energy as electrons reach relativistic 


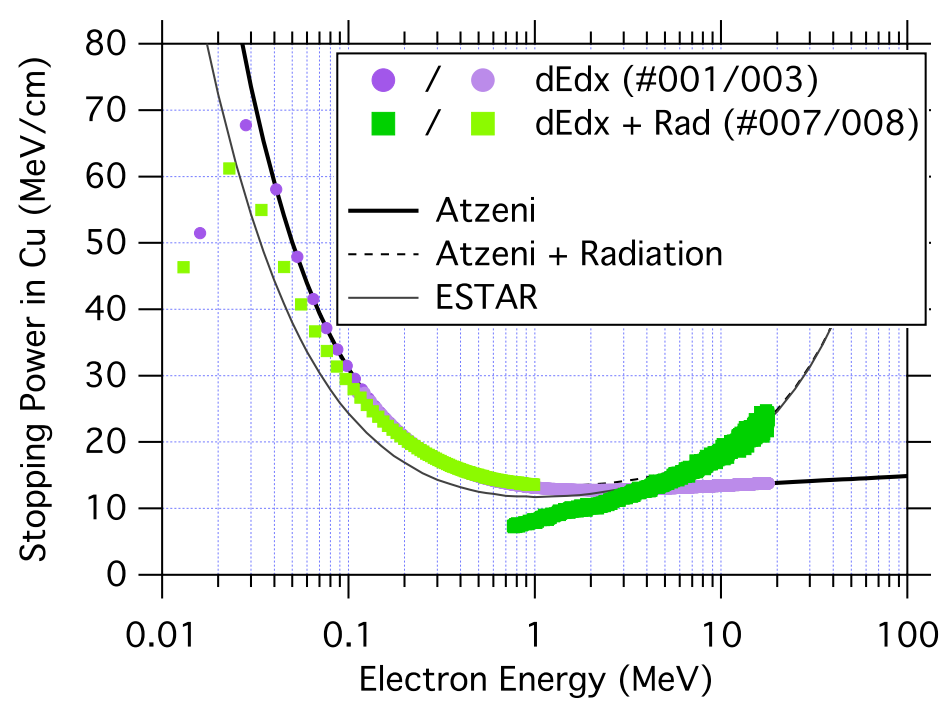

Figure 4.2: Benchmarking results of the stopping power of electrons in $\mathrm{Cu}$.

velocities. The radiative losses were included either by one of two methods. The collisional Monte-Carlo portion of the code, which replicates the $\operatorname{ITS}^{[78]}$ code can be implemented for collisions and radiation. Alternatively, LSP can be used with the deterministic radiative stopping power from Dahl. ${ }^{[7]}$ There is some difference between the radiative stopping power calculated from each model, however the difference is less than $10 \%$ which will have little affect in our analysis.

$$
\left.\frac{d \mathcal{E}}{d x}\right|_{\mathrm{rad}}=\mathcal{E}\left(\frac{716.4 \mathrm{~g} \mathrm{~cm}^{-2} \mathrm{~A}}{Z(Z+1) \ln (287 / \sqrt{Z})}\right)^{-1}
$$

It should be noted that when using the initial version of LSP, the stopping power was not calculated correctly. It was only after changes made to the source code by A. Link that the stopping power was reproduced accurately. Additionally, the Dahl radiation method was implemented by C. Orban.

\subsubsection{Benchmarking of Scattering}

The scattering process is LSP works in conjunction with the stopping power. As the stopping power changes the absolute magnitude of the momentum, the scattering changes the direction of the momentum. For electrons, LSP uses the 
small angle scattering formulation with a mean angle of $\left\langle\Theta^{2}\right\rangle$. In this method the mean angle is described by Atzeni. ${ }^{[33]}$

$$
\begin{gathered}
\frac{d\left\langle\Theta^{2}\right\rangle}{d x}=2 \frac{e^{4}}{4 \pi \epsilon_{0}^{2}} \frac{1}{p^{2} v^{2}}\left[L_{e i}+L_{e e}\right] \\
L_{e i}=Z n_{i} \times Z\left(\ln \Lambda-\frac{1-\beta^{2}}{2}\right) \\
L_{e e}=Q n_{i} \times\left(\ln \Lambda-\frac{\ln [2(\gamma+3)]+1}{2}\right)
\end{gathered}
$$

In these equations, $\ln \Lambda=\frac{4 \pi \lambda_{D}}{\lambda_{d B}}$ and $\lambda_{D}=\sqrt{\frac{k_{b} T \epsilon_{0}}{\left(Q n_{i}\right) e^{2}}}$ is the debye length. Here $L_{e i}$ and $L_{e e}$ are the scattering terms for relativistic electrons scattering off of ions and other electrons. Notice that in the equation for $L_{e e}$ that the charge state of the ions $Q$ is used. This means that scattering only occurs on free electrons and bound electrons are ignored. It is debatable where or not the bound electrons should be included in this calculation and this was not discussed by Atzeni ${ }^{[33]}$ given that they considered only fully ionized plasma. Given the fact that $L_{e i}$ scales with $Z^{2}$ and $L_{e e}$ scales with $Q$ or $Z$, we can avoid this argument by stating that we will be considering high $Z$ materials (e.g. $\mathrm{Cu}$ and $\mathrm{Au}$ ), where the electron scattering is negligible.

To verify that scattering was working correctly, the following simulation was set up in LSP with the fields disabled. Electrons were injected into a thin (1 to $10 \mu \mathrm{m}) \mathrm{Cu}$ foil, as shown in Figure 4.3. The electrons were injected with the same momentum in the forward $z$-direction and with no momentum in the transverse dimensions. After passing through the foil, the momenta of the electrons was recorded. The change in momentum is described by the value of $\Theta$. As described in the appendix, this is the ratio of forward going momentum to transverse momentum, $\cos \Theta=p_{z} / P$, where $p_{z}$ is the forward momentum and $P$ is the total momentum. Figure 4.4 shows the momentum distribution of the $18 \mathrm{MeV}$ mono-energetic electron beam after passing through a $10 \mu \mathrm{m} \mathrm{Cu}$ foil.

The root mean squared of $\Theta$ for a given thickness, $d\left\langle\Theta^{2}\right\rangle / d x$, was found for the different energies of electrons and compared with the values expected from 


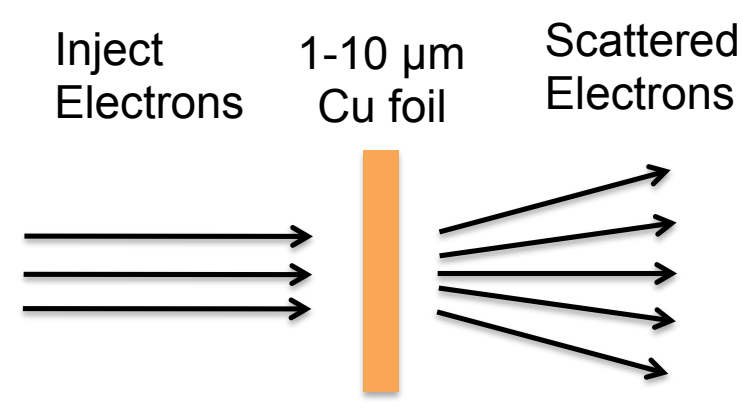

Figure 4.3: Setup of the LSP scattering benchmarking simulation.

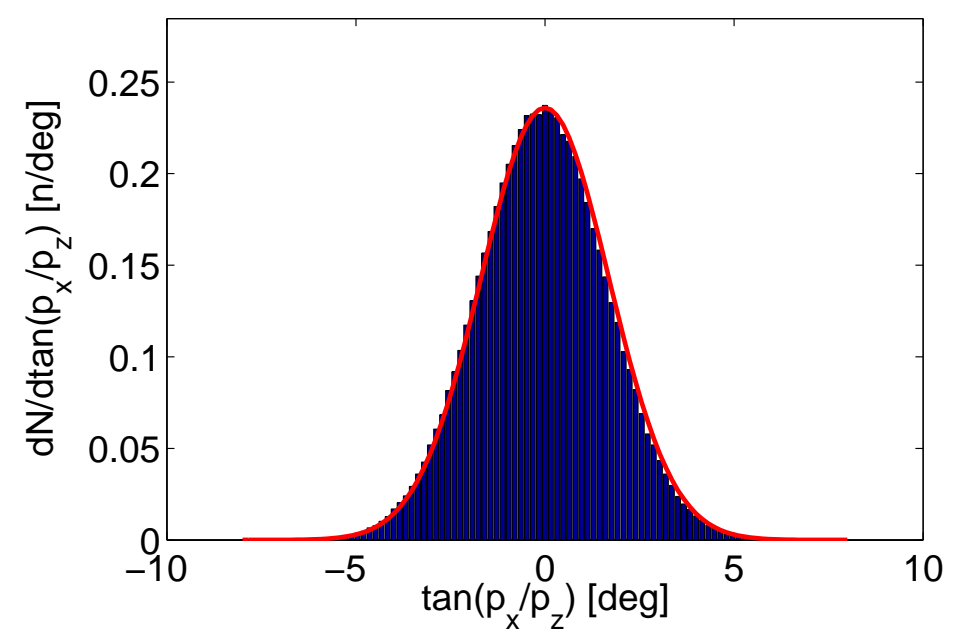

Figure 4.4: Distribution of angular momentum of $18 \mathrm{MeV}$ electrons after passing through a thin foil. The bars show a histogram and the line is a gaussian fit to the data.

equation (4.6). After some changes to the LSP source code by Dr. A. Link, the values were found to match well, as seen in Figure 4.5. It is also interesting to notice that, unlike the stopping power, the values from $\operatorname{ITS}^{[78]}$ are similar to those used in LSP. 


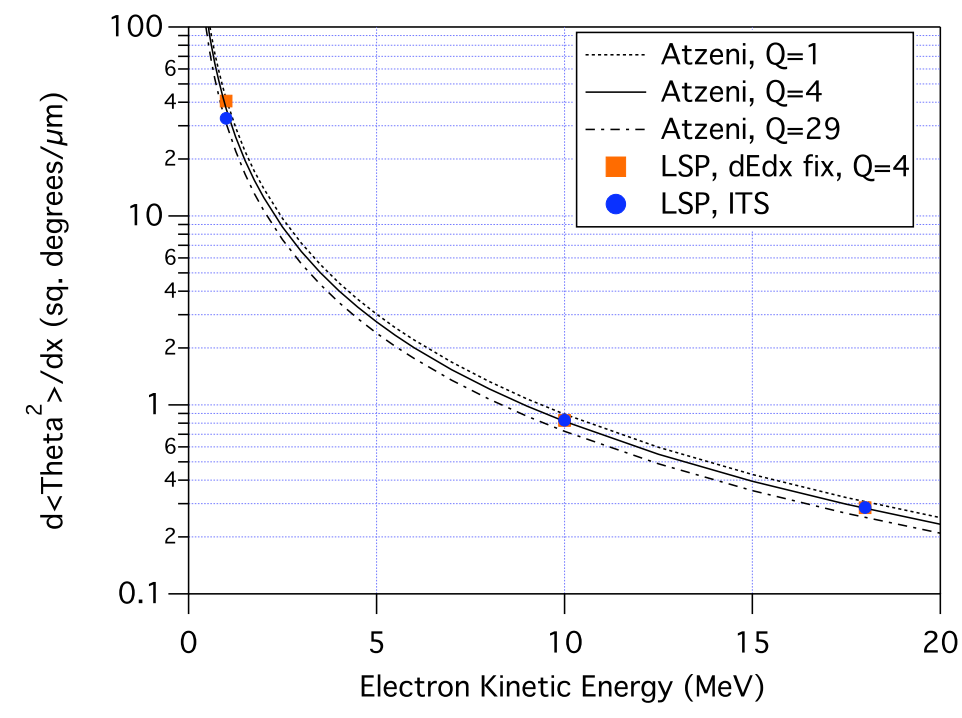

Figure 4.5: Benchmarking of the electron angular scattering in LSP.

\subsubsection{Benchmarking of $\mathbf{K}_{\alpha}$ x-ray Cross Sections}

\section{Cross sections}

A careful look at the ITS xgen source file, and the output file ${ }^{1}$, show that they use the cross section generation from a paper published in 1967 by Kolbenstvedt. ${ }^{[80]}$ One reason not to use this is that people do not seem to believe this paper, as the following was stated by Haque. ${ }^{[81]}$

The [Kolbenstvedt] model, in its original form, has the drawback in that it unusually overestimates the cross sections from the threshold to peak region and underestimate them at ultra high energies.

For this dissertation, the cross-section from a more recent paper published by Hombourger ${ }^{[71]}$ was used. ${ }^{2}$ This cross section is perhaps more accurate, but looking at the data it is not completely clear. Though it is likely that the Hombourger cross section is more accurate across the range of atomic numbers, since

\footnotetext{
${ }^{1}$ You can take a look at this by running xgen, selecting the PRINT OUT option, look in the fort.7 file to find the cross sections (which are actually probability per path length), which are in units of $\mathrm{n} \times \mathrm{cm}^{2} / \mathrm{g}$, so multiply by $\rho$ to get $\mathrm{n} / \mathrm{cm}$.

${ }^{2}$ As a note, the relativistic correction factor is incorrect $\left(G_{r}\right)$ in the Hombourger paper (equation 1.5) and the one that is correct can be found in a paper by Casnati, ${ }^{[0]}$ where the factor is $R$ (equation 0 ).
} 
these formulas are only dependent on the atomic number. It is also interesting to note that there are many other ways to calculate these cross sections [70,81, and refs within]. Since I am only interested in $\mathrm{Cu}$, I plot the $\mathrm{Cu}$ cross sections vs the available data as compiled by Lui. ${ }^{[82]}$ The two methods show a considerable difference, which is the same as noted in the previous quote.

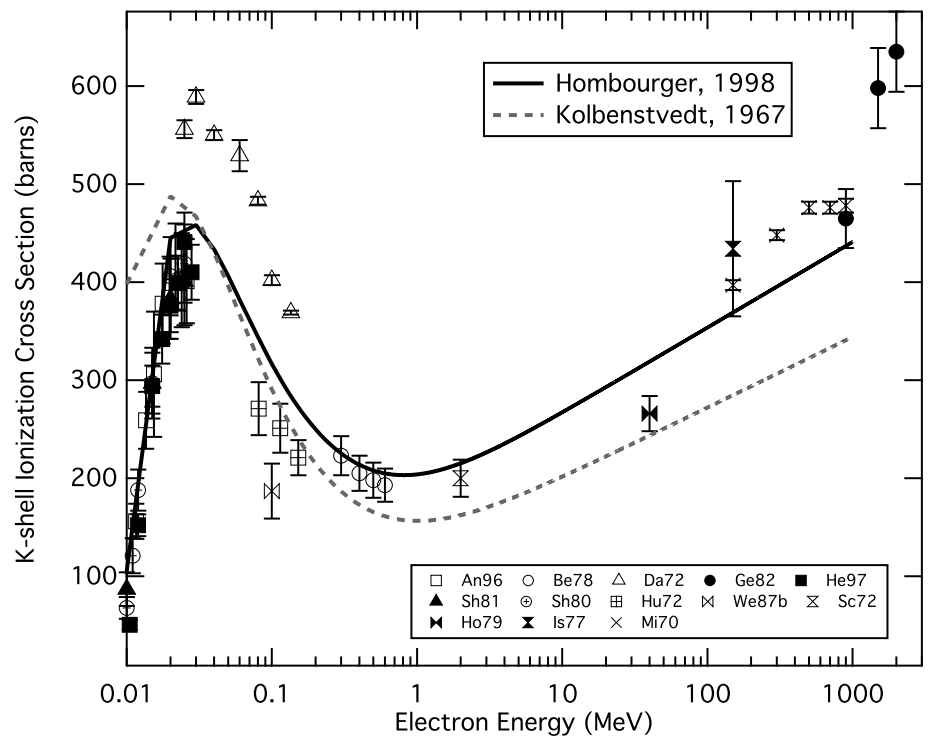

Figure 4.6: Plot of the Hombourger and Kolbenstvedt cross sections vs the experimental data. The data was taken from Ref. [82] and is named according the naming convention within the article.

\section{K $\alpha$ Generation}

To determine how electrons will generate $\mathrm{K}_{\alpha}$ emission, we look into the $\mathrm{K}_{\alpha}$ generation per energy loss, $f_{n}$.

$$
f_{n}(\mathcal{E})=\omega_{k} P_{k \alpha} n_{i} \sigma\left(\frac{d \mathcal{E}}{d x}\right)^{-1}
$$

Where $\omega_{k}$ is the probability that a k-shell photon emission will occur $\left(\mathrm{K}_{\alpha}\right.$ or $\left.\mathrm{K}_{\beta}\right), P_{k \alpha}$ is the probability that there will be a $\mathrm{K}_{\alpha}$ produced $\left(\mathrm{K}_{\alpha 1}\right.$ or $\left.\mathrm{K}_{\alpha 2}\right), n_{i}$ is the number density of ions, $\sigma$ is the cross section (as shown above) and $\frac{d \mathcal{E}}{d x}$ is the stopping power of the electron. The numbers for $\omega_{k}=0.44$ and $P_{k \alpha}=0.88$ are 
from Refs. [83,84]. The number density, $n_{i}$, is $8.48 \times 10^{22} \mathrm{n} / \mathrm{cm}^{3}$ and the stopping power is taken from NIST ESTAR tables. ${ }^{[48]}$ These are plotted in Figure 4.7.

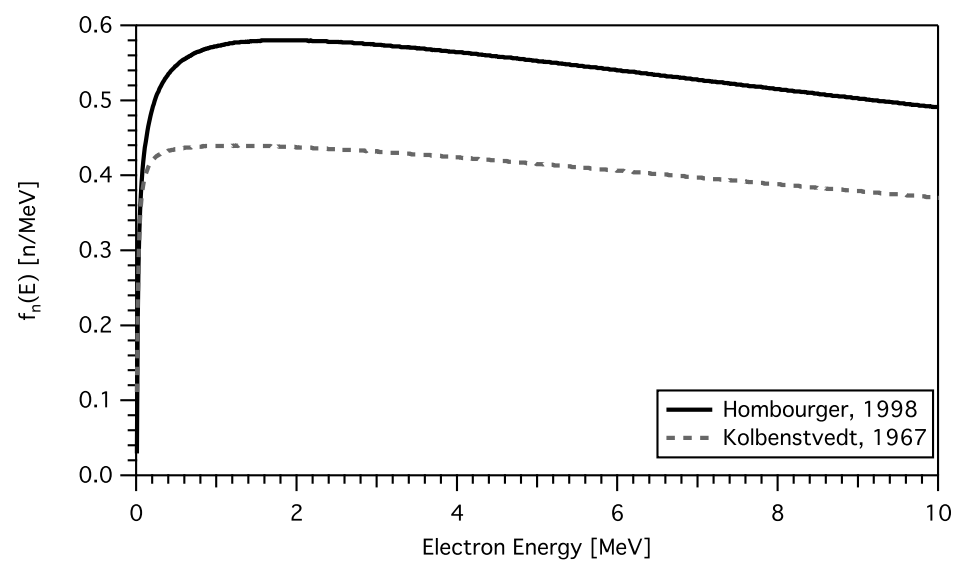

Figure 4.7: $\mathrm{K}_{\alpha}$ generation per energy loss.

Next, the total $K_{\alpha}$ energy produced from an electron as it slows in the material is examined. This only looks at energy loss through collisions. The average $\mathrm{K}_{\alpha}$ energy is $E_{\text {avg }}=P_{k \alpha 1} E_{k \alpha 1}+P_{k \alpha 2} E_{k \alpha 2}=8041 \mathrm{eV}$.

$$
E_{\text {tot }}\left(E_{\text {in }}\right)=E_{\text {avg }} \int_{0}^{E_{\text {in }}} f_{n}(\mathcal{E}) d \mathcal{E}
$$

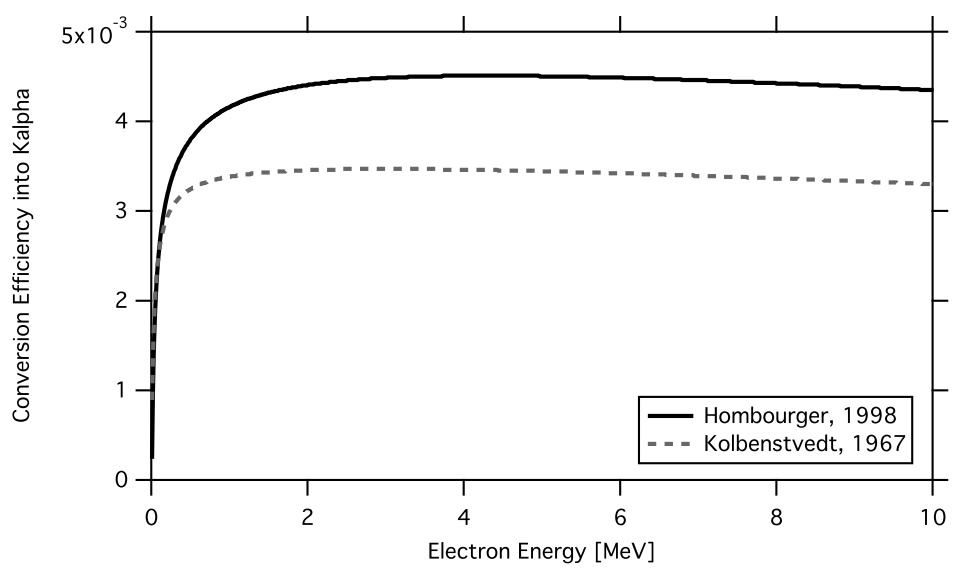

Figure 4.8: Conversion efficiency into $K_{\alpha}$ photons (linear scale).

Finally, the conversion efficiency is plotted in Figure 4.8. This is the energy produced by $K_{\alpha}$ divided by the input electron energy. This shows that the two cross section models give significantly different results. 


\subsection{Equation-of-State Models in LSP}

Another piece of physics that is included in our LSP simulations is the equation-of-state. This is a way to determine the ionization of the material as well as the amount of deposited energy that it takes to raise a material to a given temperature (i.e. the heat capacity).

The ionization of the material was included in one of two ways depending on if the material was being modeled as a fluid or kinetically. For the fluid modeling (as done in the transport simulations), an equation of state model from PROPACEOS ${ }^{[85,86]}$ was used. This models the equation-of-state of the plasma in equilibrium. It is mostly dependent on the temperature of the system, but also is affected by the density. The average ionization state, $Z^{*}$, for solid density $\mathrm{Cu}$ is shown in Figure 4.9 as a function of temperature. Also shown is the internal energy vs temperature.
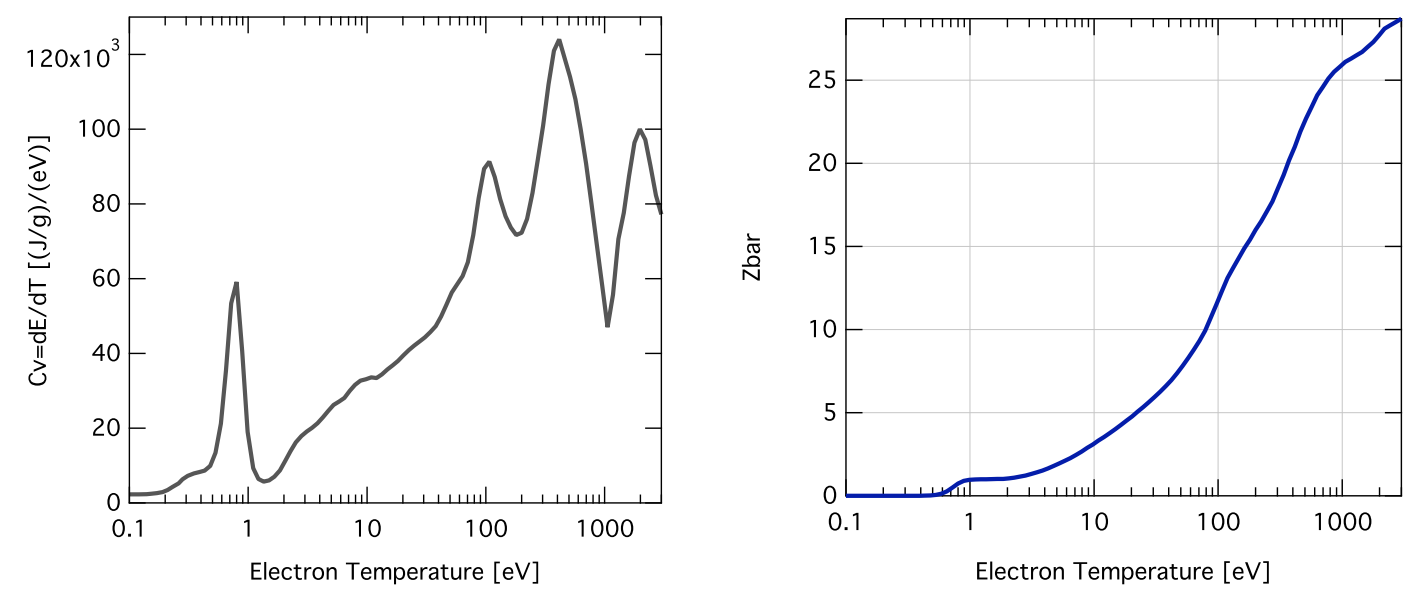

Figure 4.9: Equation-of-state in Copper. (Left) Heat capacity $C_{v}$ and (right) average ionization state $Z$ bar as a function of electron temperature.

When using kinetic particles the ADK ${ }^{[59]}$ field ionization model was used, this model is described in more detail in Section 2.2.1. This model is used in the Laser-Plasma Interaction (LPI) simulations. In these simulations, the most important physics occurs in the relatively low density $\left(10^{19}\right.$ to $\left.10^{22} \mathrm{n} / \mathrm{cm}^{3}\right)$ plasma where the laser interacts. Since the density is low, collisions between particles are 
rare and the field is the dominate component to ionization.

\subsection{Proton Layer Benchmarking}

As discussed previously, the charging of the wire plays a large role in how the system evolves. Thus including all of the physics relevant to this charging is important. Previous work has studied the build up of electric charge along a vacuum interface due to electron acceleration through a target. ${ }^{[46]}$ This work showed that LSP models the electric fields correctly as compared to analytical solutions.

For the simulations for this dissertation a proton layer on the edge of the wire are included. The protons exist in the simulation due to contaminants in the air and in the target chamber. These leave a $\sim 10 \mathrm{~nm}$ layer of hydrocarbons (mostly hydrogen) on any target. We followed the paper by Allen ${ }^{[87]}$ to estimate the amount of contaminants that should be present on the $\mathrm{Cu}$. In reality, there is only a few nm of material, however this is not feasible to model in our simulations and we generally use $1 \mu \mathrm{m}$ cells for the transport simulations. Most simulations modeling this contaminant layer do not use nanometer sized cells, however many use cells of $0.1 \mu \mathrm{m}$. In order to make sure that our simulations ran correctly with $1 \mu \mathrm{m}$ cells, we ran a test case comparing 0.1 to $1 \mu \mathrm{m}$ cells, while using the same number of macroparticle protons. The proton densities of these simulations are shown in Figure 4.10. In this test simulation, electrons are injected into a thin $\mathrm{Cu}$ foil with protons on the rear side of the foil. The $0.1 \mu \mathrm{m}$ cell simulation shows a conversion efficiency of protons from electons of $0.36 \%$ and the $1 \mu \mathrm{m}$ cell simulations shows $0.31 \%$. This close match shows that the use of $1 \mu \mathrm{m}$ cells is appropriate for our simulations.

The benchmarking performed in this chapter gives confidence that important features of LSP, such as collisions and $\mathrm{K}_{\alpha}$ cross sections, are implemented correctly in the code. As these simulations are used to interpret the experimental data and relate it to electron spectra, it is very important that the code is well benchmarked. 

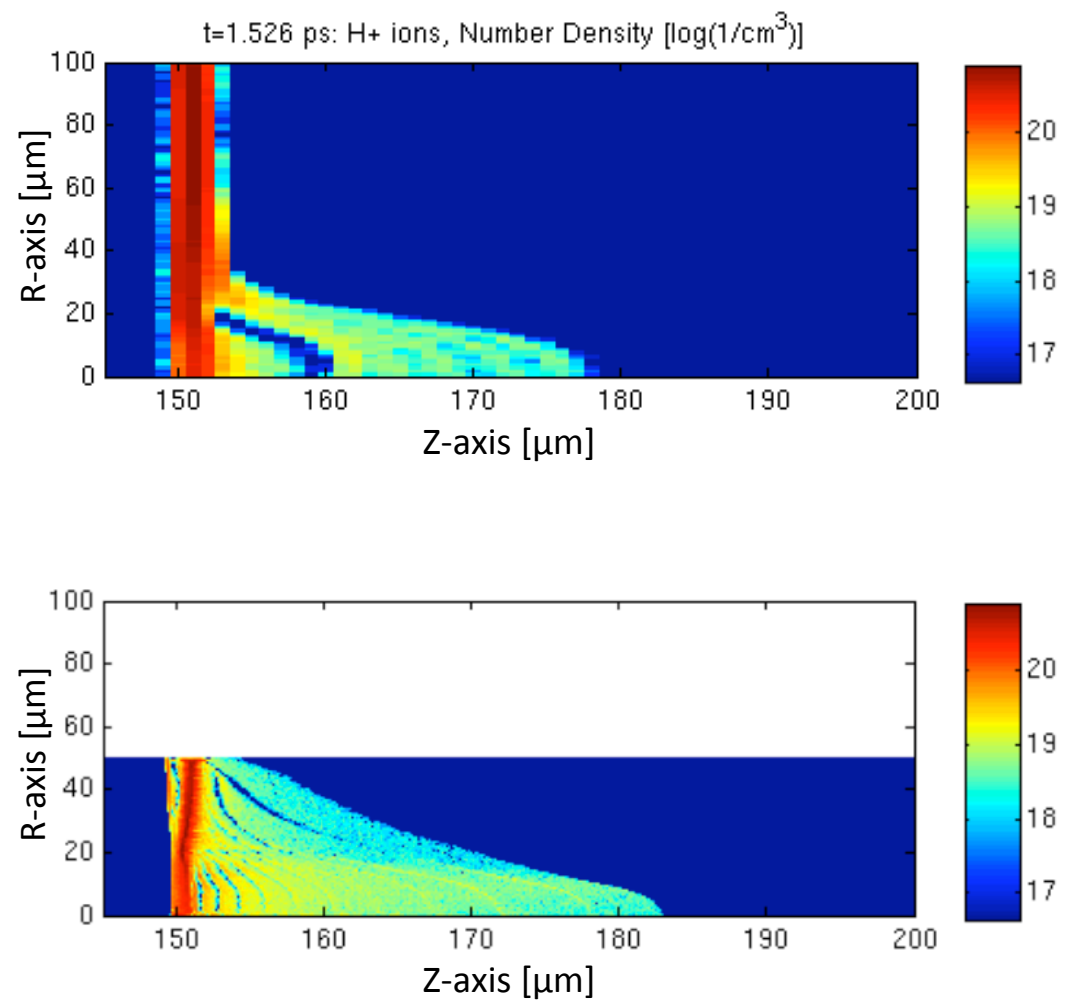

Figure 4.10: Comparison of cell size for proton acceleration from a thin foil. (Top) Cell size of $1 \mu \mathrm{m}$ with a conversion efficiency is $0.36 \%$. (Bottom) Cell size of $0.1 \mu \mathrm{m}$ with a conversion efficiency is $0.31 \%$. 


\section{Cone-Wire Experiments}

\section{$5.1 \quad$ Experimental Setup}

The purpose of this dissertation is to increase our understanding of electrons accelerated by short-pulse laser interactions, especially in the context of the fast ignition (FI) scheme. To achieve this, a cone-wire target was used as a diagnostic to characterize the electron spectra for different laser parameters. This target consists of a hollow $\mathrm{Au}$ cone attached to a $\mathrm{Cu}$ wire, as shown in Figure 5.1. The cone is $20 \mu \mathrm{m}$ thick around the sides and $10 \mu \mathrm{m}$ thick at the tip. The cone tip is $30 \mu \mathrm{m}$ wide with a $30^{\circ}$ opening angle. The cone is $1 \mathrm{~mm}$ long. The $\mathrm{Cu}$ wire is attached to the outer cone-tip with UV curing glue and is $1.5 \mathrm{~mm}$ long with a 40 $\mu \mathrm{m}$ diameter. The laser is incident on the inner cone tip. This causes electrons to be accelerated through the $\mathrm{Au}$ cone tip and into the $\mathrm{Cu}$ wire.

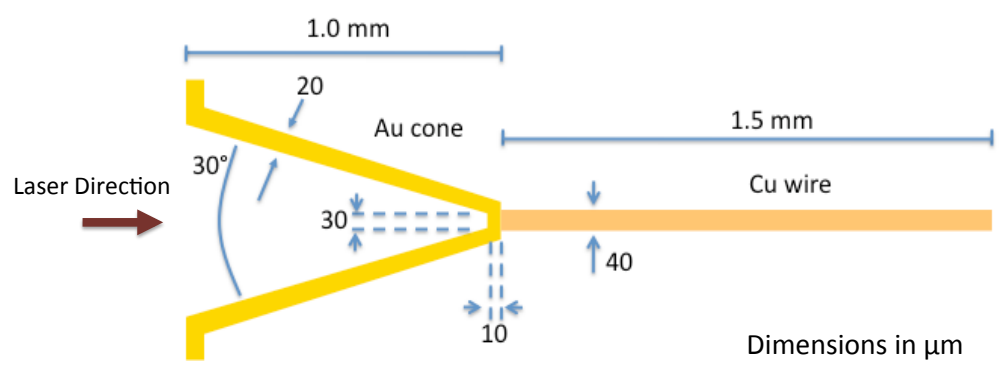

Figure 5.1: Dimensions of the cone-wire targets used in our experiments to diagnose the electron spectra of high-intensity lasers. The laser comes from the left in this diagram and is incident on the inner cone tip.

As electrons pass through the $\mathrm{Cu}$ wire they cause $\mathrm{K}_{\alpha}$ photons to be emitted and these are collected by a calibrated spectrometer and a spherical imager, as 
discussed in Section 3.3 and 3.4. The $\mathrm{Cu} \mathrm{K}_{\alpha}$ photons have an energy of $8 \mathrm{keV}$, which corresponds to an attenuation length of $22 \mu \mathrm{m}$ in solid density Cu. For this reason, the wire radius was chosen to be relatively thin. We assume that the wire is uniformly emitting across a given circular cross-section; this is expected from the scattering in the thin wire and is verified through simulations. This allows us to determine the opacity of the wire as a function of the view-angle of our imager as derived in the Appendix. The length of the wire was chosen to be as long as possible while fitting within the field of view of the $\mathrm{K}_{\alpha}$ imager. This allowed us to observe the full $\mathrm{K}_{\alpha}$ signal from electrons within the wire.

The dimensions of the cone were chosen to be similar to the cone used for fullscale FI implosions. Thus making the conditions of the interaction relevant in an FI context. The Au cones were manufactured at Rutherford Appleton Laboratory in the UK for the Titan experiments and at General Atomics for the Trident experiment. The attachment of the cones to the wire was performed for both experiments at General Atomics in San Diego. Care was taken to use as little glue as possible and to align the wires directly in the middle of the cone. Since these are relatively difficult to assemble, the targets were all characterized before use in our experiments and verified to be in good condition before use. Figure 5.2 shows a sample of the characterization of these cone-wires.
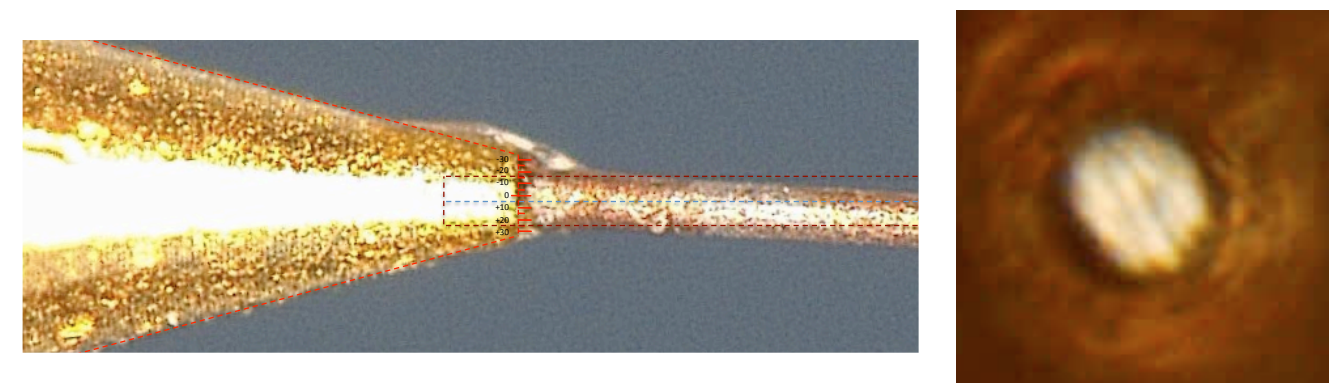

Figure 5.2: Photographs of the cone-wires. (left) Side on view of the wire and cone connections and (right) interior cone tip.

These targets were shot at both the Trident and Titan laser facilities. The laser focal spot sizes were optimized to obtain the smallest focal spot possible, as shown in the Section 3.1.2, and was kept constant for all shots. The pulse length 
was also kept as short as possible. To explore a range of intensities, the energy of the lasers was varied from 24 to 75 joules on Trident and from 16 to 160 joules on Titan. This covered an peak intensity range from $10^{19}$ to $10^{20} \mathrm{~W} / \mathrm{cm}^{2}$.

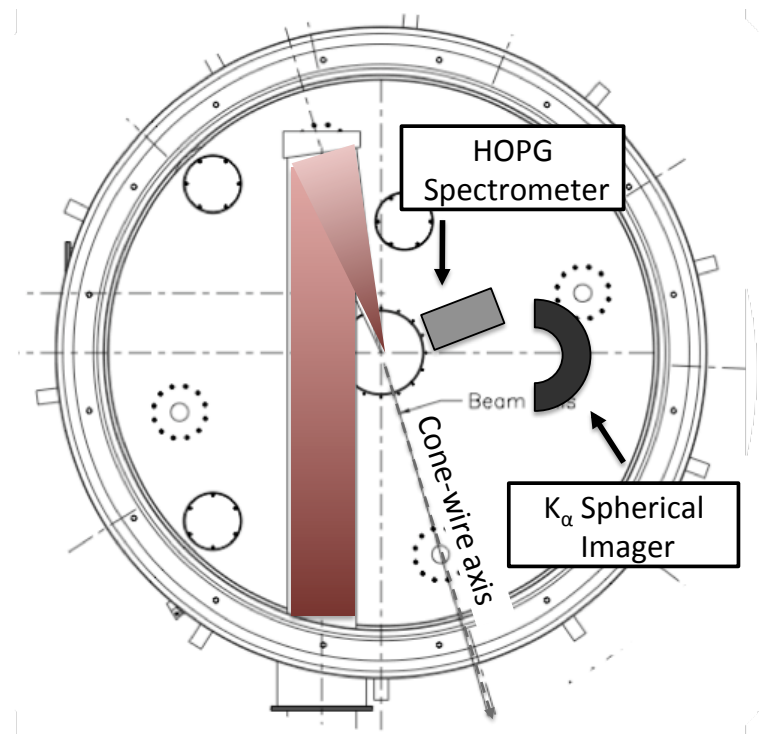

Figure 5.3: Diagram of the experimental setup for laser experiments using conewire targets. The setup was similar on both the Trident and Titan laser systems. The laser was used at normal incidence to the cone tip. The HOPG spectrometer and $\mathrm{K}_{\alpha}$ imager were the main diagnostics.

As discussed in Section 3.1.3, the major difference between the Trident and Titan laser systems is in the amount of prepulse laser energy prior to the main laser pulse. The prepulse can create a large amount of plasma that moves the critical surface of the target further away from the initial position. This preplasma can also cause non-linear effects, such as relativistic self-focusing and filamentation, which will change the way that the laser interacts and accelerates electrons. The Trident laser has a much lower amount of prepulse energy than Titan, thus we expect the amount of preplasma to be significantly reduced.

The cone-wire experiments were set up with the laser pointing directly down the cone so that it impacted the tip of the cone at normal incidence. We measured the total amount of $\mathrm{K}_{\alpha}$ radiation that was emitted by the wire using a calibrated HOPG spectrometer at $17^{\circ}$ from the wire normal. Since the $\mathrm{K}_{\alpha}$ cross section is 
relatively constant for electron energies from 0.5 to $10 \mathrm{MeV}$, this diagnostic gives us a measurement of the total energy that is coupled into the wire. To obtain an image of the $\mathrm{Cu} \mathrm{K} \mathrm{K}_{\alpha}$ x-rays emitted from the $\mathrm{Cu}$ wire, we used the spherical crystal imager, which reflects x-ray energies of $8048 \mathrm{eV}$ with a $\sim 6 \mathrm{eV}$ bandwidth. This diagnostic was placed at $19^{\circ}$ from the wire normal. A diagram of the experimental setup is shown in Figure 5.3.

\subsection{Coupling Efficiency}

In Fast Ignition, electrons will be accelerated by a laser into the fuel to provide heating. A cone is used to create a plasma-free path for this laser to propagate, however the electrons much get past the tip of the cone to deposit energy into the core of the fuel. The wire placed at the end of the cone is a diagnostic analog to the imploded fuel core in Fast Ignition. The amount of $\mathrm{K}_{\alpha}$ radiation that is created by electrons is related to the amount of energy that is deposited in the wire, as shown in Section 4.3.3.

Thus we use the amount of $\mathrm{K}_{\alpha}$ generated by the cone to diagnose the amount of energy lost within the wire. This value is corrected for the opacity of $\mathrm{Cu}$ to 8 keV electrons, which are assumed to be emitted uniformly and isotropically within the wire. This assumption is made due to the small radius of the wire and is corroborated in simulations. The opacity calculation is shown analytically in the Appendix.

Once we have the opacity corrected $K_{\alpha}$ values we divide these by the total laser energy to get the conversion efficiency into $K_{\alpha}$ photons. This is called the $\mathrm{K}_{\alpha}$ coupling efficiency and is plotted against laser intensity in in Figure 5.4. The data shows no correlation between coupling efficiency of electrons into the wire and intensity for a given laser system. However, there is still a variation of the coupling efficiency that is around $50 \%$ for the same laser at similar intensities.

There is an definite difference in the shot-averaged coupling efficiency between Trident, at $0.00907 \%$, and Titan, at $0.00340 \%$. These comparisons are done of a range of intensities, but the prepulse energy is kept as low as possible. By 


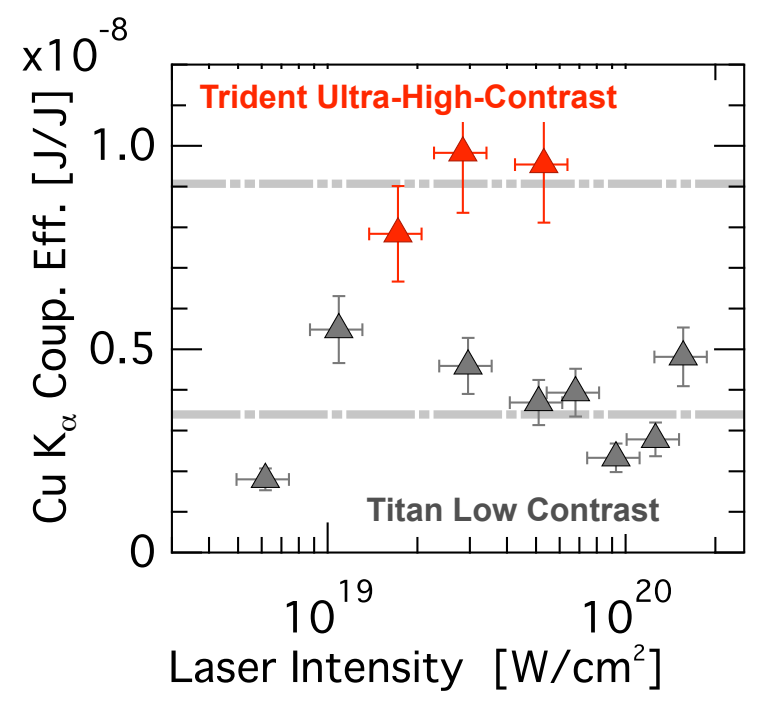

Figure 5.4: K $\alpha$ coupling efficiency on the Trident and Titan laser systems.

comparing the averages on both facilities, we see that there is a $2.7 \mathrm{x}$ increase in coupling efficiency on Trident as compared to Titan. This difference is due to the different amount of preplasma that is present in the cone due to different amount of prepulse laser energy incident on the tip prior to the main pulse of the laser. In the case of Titan, there is a large amount of preplasma filling the cone, which pushed back the critical surface and implies that electrons are accelerated further away from the cone tip, and will not be directed toward the wire.

This work is an extension of work that we have done previously ${ }^{[41,43]}$ as shown in Figure 5.5. In these past experiments, we had injected an additional amount of prepulse into the cone to determine its effect on the coupling. As can been seen in Figure 5.5, there is a dramatic decrease in coupling as the prepulse energy is increased. However, the difference is that on Trident, for ultra-highcontrast, we move into a regime where there very little prepulse. Instead, any preplasma that is created should be due to the rising-time of the laser, which occurs on time scales that are less than 0.1 ns. We can think of this data as perhaps the best coupling that one could expect, because the electrons should be injected at distances that are quite close to the cone-tip. 


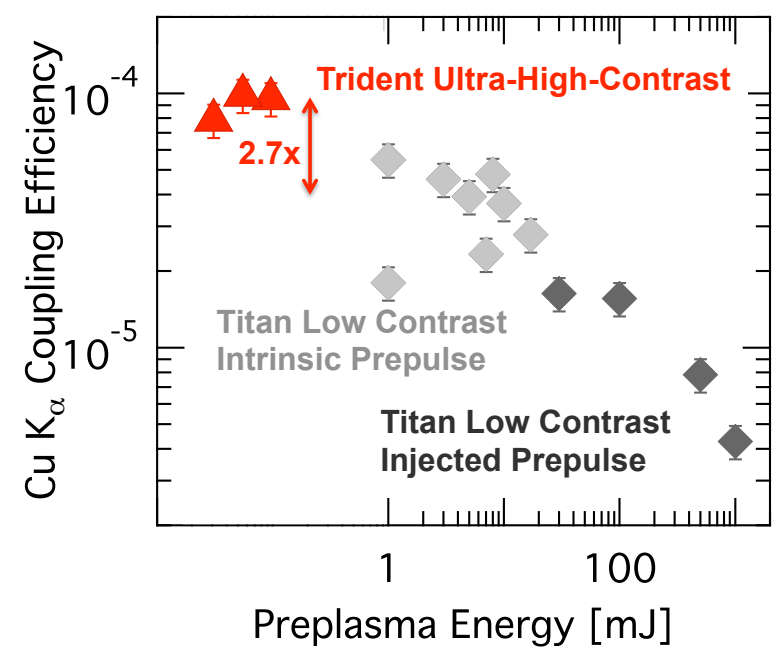

Figure 5.5: K $\alpha$ coupling efficiency on the Trident and Titan laser systems versus prepulse level. The Trident high contrast (purple triangles) and Titan Intrinsic Low Constrast (gray diamond) are from this work. The Titan injected Prepulse (red triangles) is also from the Titan experiment, but has been previously been published $^{[41]}$ and thus is not discussed further in this text.

\section{$5.3 \quad \mathrm{~K}_{\alpha}$ Falloff Lengths}

In order to assess the spectrum of the accelerated electrons we use an image of the $\mathrm{Cu} \mathrm{K} \mathrm{K}_{\alpha}$ emitted from the $\mathrm{Cu}$ wire. By looking at the extent of the $\mathrm{K}_{\alpha}$ emission we can infer the energies of the electrons. This is because the electrons with more energy will travel further in the wire and thus will emit $\mathrm{K}_{\alpha}$ radiation further into the wire. Figure 5.6 shows an image of the raw data from one shot on both Trident and Titan. This raw image was smoothed slightly using a median filter to remove noise. The images have also been background subtracted with a background taken from above and below the image of the wire. The data from Trident was acquired using Imaging Plates (IPs), while Titan used a CCD camera. Notice that the width of the wire in the Trident data appears slightly larger, this is due to the poorer resolution of the data taken on the Trident laser.

These images were averaged in the transverse direction (radially along the wire) and then a lineout was taken longitudinally. The lineout was normalized to 


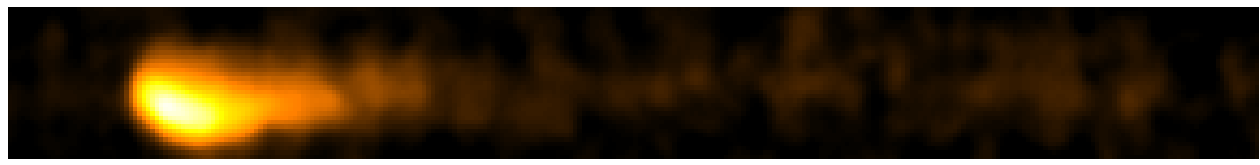

(a) Image of $\mathrm{K}_{\alpha}$ radiation from Trident shot 21590 with $75 \mathrm{~J}$ of laser energy.

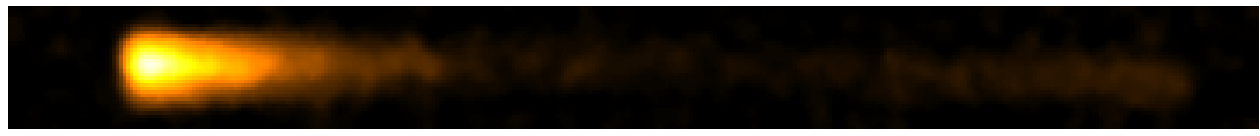

(b) Image of $\mathrm{K}_{\alpha}$ radiation from Titan shot 20090826s01 with $161 \mathrm{~J}$ of laser energy.

Figure 5.6: Raw images of the $\mathrm{K} \alpha$ radiation coming from the $\mathrm{Cu}$ wires. The colormaps are normalized to the peak signal.

total amount of $\mathrm{K}_{\alpha}$ on the shot as measured by the HOPG spectrometer. The lineouts corresponding to the raw images in Figure 5.6 are shown in Figure 5.7.

When discussing the cone-wires, it is useful to identify the different features of the lineouts, because different portions of the electron spectrum are responsible for creating the different parts. There are four important features: 1) the peak of the emission, 2) the slope of the initial falloff, 3) the flat portion in the middle, and 4) the bump at the end of the wire.

The peak of the emission at the beginning of the wire is the highest part of the lineout. While this seems useful in the analysis of electrons it is susceptible to many unrelated factors, and thus must be interpreted with care. The abrupt change in signal at the peak means that the height of the observed signal will be affected by the resolution of the imaging system. Uncertainty in this resolution will lead to uncertainty in the peak. Additionally, there is the issue of temperature dependent shifting of the $K_{\alpha}$ lines, which will reduce the recorded signal as discussed in Section 3.4. Since the beginning of the wire has the largest current of electrons, it will heat up the most and thus may be the most affected by the shifting of the lines. Thus the height of the peak is susceptible to factors other than the amount of $\mathrm{K}_{\alpha}$ emission and thus must be interpreted with care.

The initial falloff of electrons is very important to the study of the electron spectrum. This falloff extends for about $500 \mu \mathrm{m}$ from the peak of the emission. Here, unlike the peak of emission, the variation in signal is continuous, and there- 

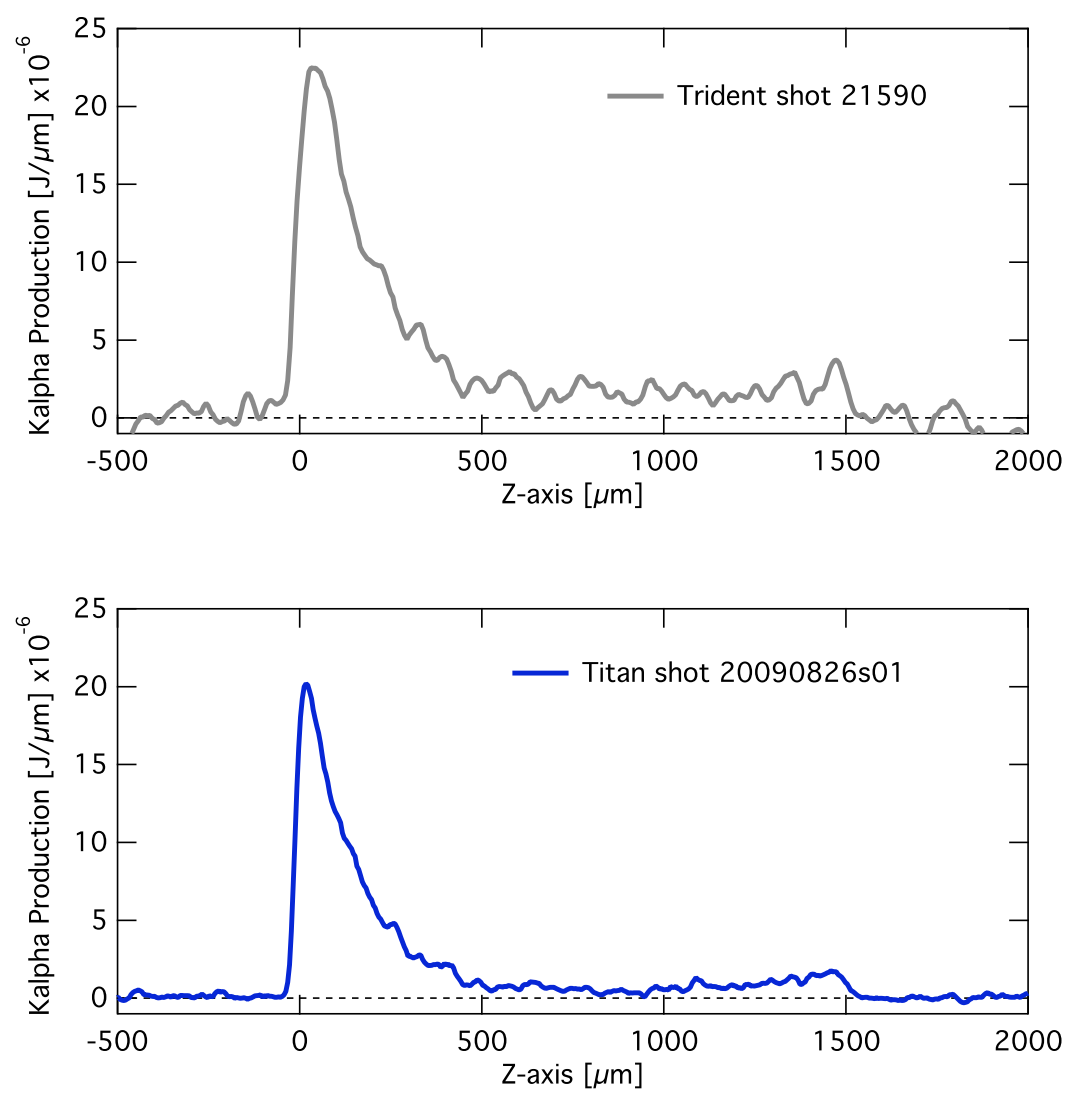

Figure 5.7: Lineouts of the $\mathrm{K}_{\alpha}$ radiation coming from the $\mathrm{Cu}$ wires from (top) the Trident laser at 75 and (bottom) the Titan laser at $161 \mathrm{~J}$.

fore is less susceptible to the resolution of our imager diagnostic. This slope will be influenced by the energy of electrons in the wire. Since more energetic electrons can travel further in the wire they will cause the emission to falloff over longer distances than less energetic electrons, and thus we can use the distance of the initial falloff to infer the energy of electrons traveling through the wire. As we will show shortly, this portion of the wire is mainly a constraint on the 0.2 to $2 \mathrm{MeV}$ electrons. This is because electrons below $0.2 \mathrm{MeV}$ do not have enough energy to get past the cone tip and electrons above $2 \mathrm{MeV}$ have falloff distances well above $500 \mu \mathrm{m}$.

The next feature of these lineouts is the flat portion along the middle section, which occurs from about 500 to $1000 \mu \mathrm{m}$ in the wire. This portion of the 
emission is quite flat and is due to electrons not experiencing much energy loss within the $1.5 \mathrm{~mm}$ of the wire and thus their emission is relatively constant through out the entire wire. These electrons have energies greater than $\sim 2 \mathrm{MeV}$ and are out of the energy range of the cone-wire diagnostic.

In many of the lineouts of the wire emission, there is a secondary peak, or bump, at the end of the wire. This is most noticeable on the Titan lineout in Figure 5.7. The height of this bump is often more than twice the height of the emission along the flat middle portion of the wire. In some respects, this is the most mysterious part of the wire, and understanding how this is created will require modeling of the development of both electric and magnetic fields in the wire that play a large role in the transport of electrons. We will discuss this in detail in the Transport Section, and show that the physics that creates this bump is complicated and highly non-linear. Thus, while interesting, the generation of this bump does not tell us much about the energies of the electrons that created it.

Since, the most important constraint on the electrons is the initial falloff of electrons, it is useful to quantify this slope for comparison across different laser conditions. To do this, an exponential slope was fit to the wire $f(z)=\exp \left[-z / L_{\mathrm{f}}\right]$, were $z$ is the dimension across the lineout and $L_{\mathrm{f}}$ is the falloff along the wire. This was done by three different methods to get an understanding of the uncertainty within these fits. The first method was to use two points separated by a given distance and calculate the amount of signal drop-off between them. Care was take to use points that were 50-100 $\mu \mathrm{m}$ away from the peak of the emission to avoid any influence of the resolution of the imager. The second method was to fit all of the points some distance from the peak with an exponential slope. Again, here the region near the peak was avoided. Finally, the initital falloff was fit with an exponential decay function convolved with a gaussian function to represent the spatial resolution of our imaging system. This allowed us to fit both the exponential falloff as well as the peak of the emission. These three techniques were averaged to determine the falloff and the standard deviation was taken to quantify the uncertainty. 
The falloffs are plotted as a function of laser intensity in Figure 5.8. We see a strong dependence of the falloff on laser intensity at the individual laser systems. This is expected, as previous work has suggested that the energy of electrons increases as the laser intensity increases, due to the so-called $\mathbf{j} \times \mathbf{B}$ heating mechanism $^{[44]}$ discussed in Section 2.3.3.

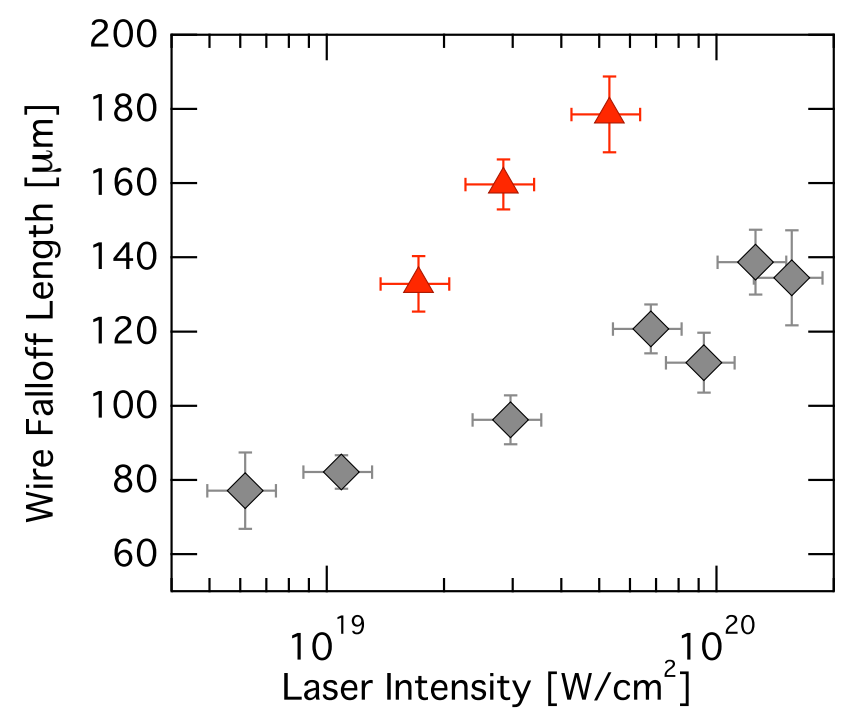

Figure 5.8: $\mathrm{K} \alpha$ falloffs on the high-contrast Trident (red triangles) and lowcontrast Titan (gray diamonds) laser systems.

Along with the variation with intensity, there is a strong dependence of the falloff distance with changes in the prepulse levels. At high-contrast on Trident there is a longer falloff distance than at low-contrast on Titan. From this information, we can infer that the electron temperature within the wire is hotter at high-contrast than at low-contrast, implying that preplasma decreases the electron temperature. This is not consistent with previous work ${ }^{[88]}$ showing the opposite trend. This may be due to the fact that we measure only electrons that enter into the wire. Electrons with higher energies may indeed be created at low-contrast, but our data suggests that the ones that enter into the wire have lower energies. As we will see later, there are strong electric fields that develop in the cone-wire that cause electrons to be trapped within the system. Electrons with higher energy have more kinetic energy to escape the electric fields and since they are accelerated 
further from the cone tip in the low-contrast case, perhaps they are more likely to leave the system. This is a puzzle that we hope to resolve by modeling both the laser-interaction and electron-transport physics.

Our experiments show a definite dependence in $\mathrm{K}_{\alpha}$ coupling efficiency and falloff along the wire on changes in both the laser intensity and the amount of prepulse. This information will allow us to ascertain the electron spectrum and conversion efficiency once we determine the relationship between these electrons and the data that has been collected. To unwrap the electron spectrum, we will start with simple models to get a basic understanding of how these wires respond to electrons in Chapter 6. Then we model the full transport of electrons in Chapter 7 though the wire with inclusion of electric and magnetic fields, equation-of-state and other important physcis. Finally, Chapter 8 will model the laser-interaction itself and compare this to the experimental data. 


\section{Cone-Wires as Diagnostics for Hot Electrons}

\subsection{Sensitivity Range to Electron Energies}

The cone-wire targets can become very complicated systems with electric and magnetic fields that strongly influence the transport of electrons through the system. However, simple collisional models of electrons transporting through the target and neglecting the fields can be useful to get an initial understanding of the dynamics of the targets. Simulations were run using the hybrid-PIC code LSP. ${ }^{[55]}$

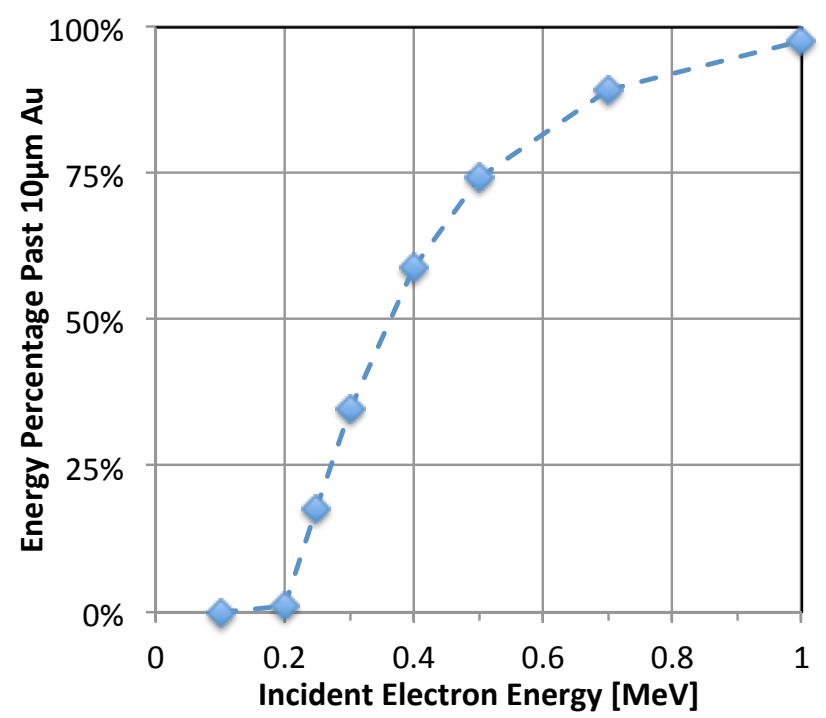

Figure 6.1: Energy percentage remaining in mono-energetic electrons after passing through a $10 \mu \mathrm{m}$ Au block. 
To understand the effect of stopping from the $10 \mu \mathrm{m}$ Au cone tip, the collisional stopping power of electrons in Au was integrated over $100 \mu \mathrm{m}$ (negliecting collective effects). The results are shown in Figure 6.1. A strong dependence of energy is observed; where electrons below $0.2 \mathrm{MeV}$ are completely stopped in the cone, but electrons above $1 \mathrm{MeV}$ only lose a small portion of their energy.

To determine the upper limit of electron energy sensitivity, electrons are injected into an infinite, solid $\mathrm{Cu}$ block. The $\mathrm{Cu}$ block is an approximation of the $\mathrm{Cu}$ wire, assuming that all electrons are reflected specularly from the wire boundaries, as would be the case if there were very strong electric fields. For these simulations, electrons are injected with a $45^{\circ} \mathrm{FWHM}$ gaussian angular distribution, which is in agreement with previous simulations. ${ }^{[7]}$

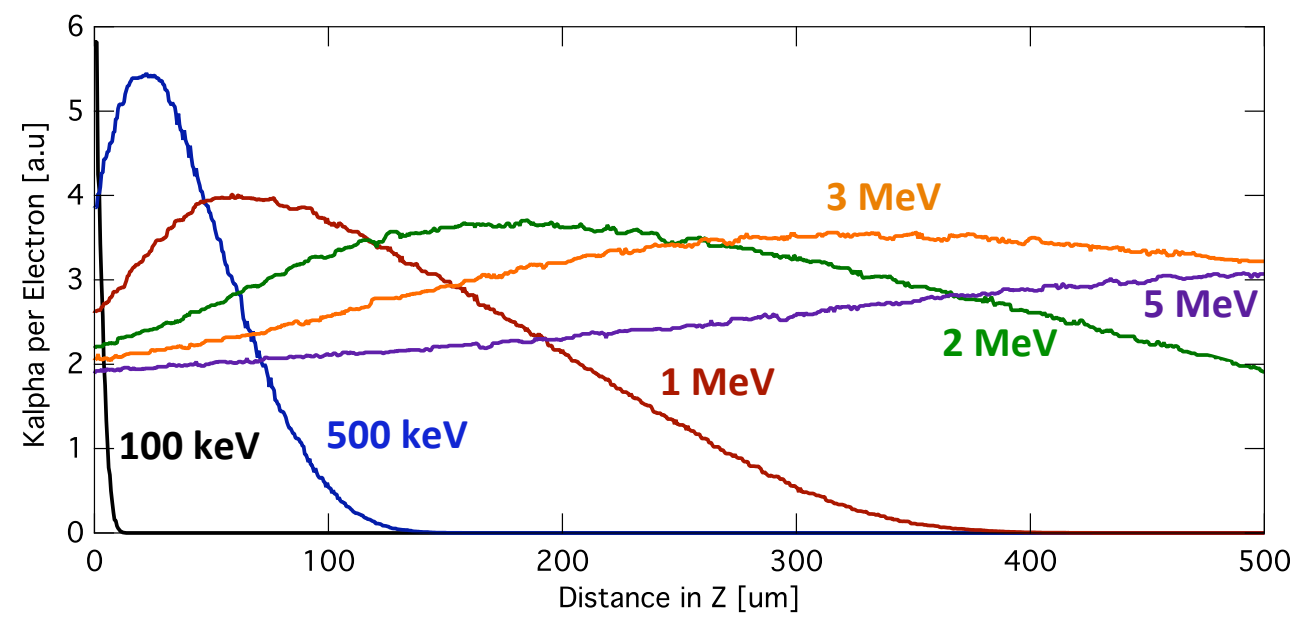

Figure 6.2: $\mathrm{Cu} \mathrm{K}_{\alpha}$ emission versus distance from mono-energetic electrons in an infinite $\mathrm{Cu}$ block.

To get an upper boundary on the energy discrimination of the wires, we use this simple model and inject mono-energetic electrons. As these electrons travel through the wire they cause $\mathrm{K}_{\alpha}$ photons to be emitted, which are measured by the calculation. The results are shown in Figure 6.2. This shows a large difference in the falloff between $0.5 \mathrm{MeV}$ electrons and $1 \mathrm{MeV}$ electrons, thus because the slope is so different it is possible for us to distinguish between the two energies. However, for energies greater than about $2 \mathrm{MeV}$ one can see that there is little different in the slopes of the $K_{\alpha}$ profiles. This means that it is difficult to distinguish the 
different between an electron of $2 \mathrm{MeV}$ and one of $3 \mathrm{MeV}$. To summarize, the cone-wire targets have a good sensitivity to electrons with kinetic energies from 0.2 to $2 \mathrm{MeV}$.

\subsection{Relationship between $\mathrm{K}_{\alpha}$ Emission and Total Electron Energy}

In this work, we are interested in determining the total amount of energy that the electrons receive from the laser. Thus it is important to understand the relationship between the total amount of $\mathrm{K}_{\alpha}$ generated and the energy lost within the wire. As noted in the diagnostics chapter, the $\mathrm{K}_{\alpha}$ cross-section is relatively flat for electron energies above $0.1 \mathrm{MeV}$. The $\mathrm{K}_{\alpha}$ cross-section $^{[70,71]}$ is plotted along with the stopping power of $\mathrm{Cu}^{[33]}$ in Figure 6.3.

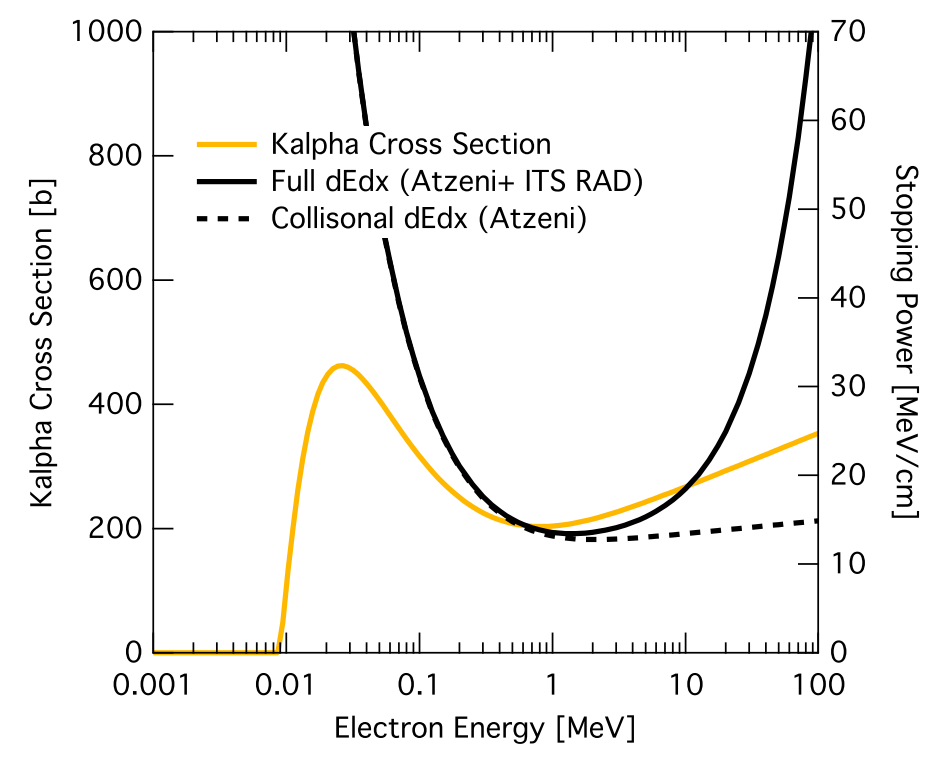

Figure 6.3: Cross-section for k-shell ionization and stopping power for electrons in solid density $\mathrm{Cu}$.

To quantify the relationship between stopping power and $\mathrm{K}_{\alpha}$ generation, we explore two difference cases. The first case is the instantaneous energy loss versus the instantaneous $\mathrm{K}_{\alpha}$ generation, this is similar to an infinitely thin foil. As 
well as looking at all of the $\mathrm{K}_{\alpha}$ generation and energy loss for all electrons, we also look at the energy loss when we exclude electrons below $50 \mathrm{keV}$. The reason for this is that these electrons generate no $\mathrm{K}_{\alpha}$ radiation and thus skews the radiation fraction; additionally in our experiment, these electrons will be stopped within the $\mathrm{Au}$ cone. The second case is when electrons lose all of their energy in the $\mathrm{Cu}$ block, which is the case of an infinitely thick foil. The results are plotted in Figure 6.4. Notice that the conversion efficiencies are relatively flat from a 0.5 to $10 \mathrm{MeV}$ exponential distributions. This means that $\mathrm{K}_{\alpha}$ emission measurement is a relatively linear diagnostic to determine the amount of energy lost within the material. This is a powerful conclusion, since it states that the energy loss within the wire can be calculated directly from the experimental data with very little model dependence. However, one must be careful with this statement, because the amount of energy lost within the wire may not be correlated to the total electron energy accelerated by the laser. Electron energy can be lost in many other ways, such as escaping in vacuum or loss within the Au cone.

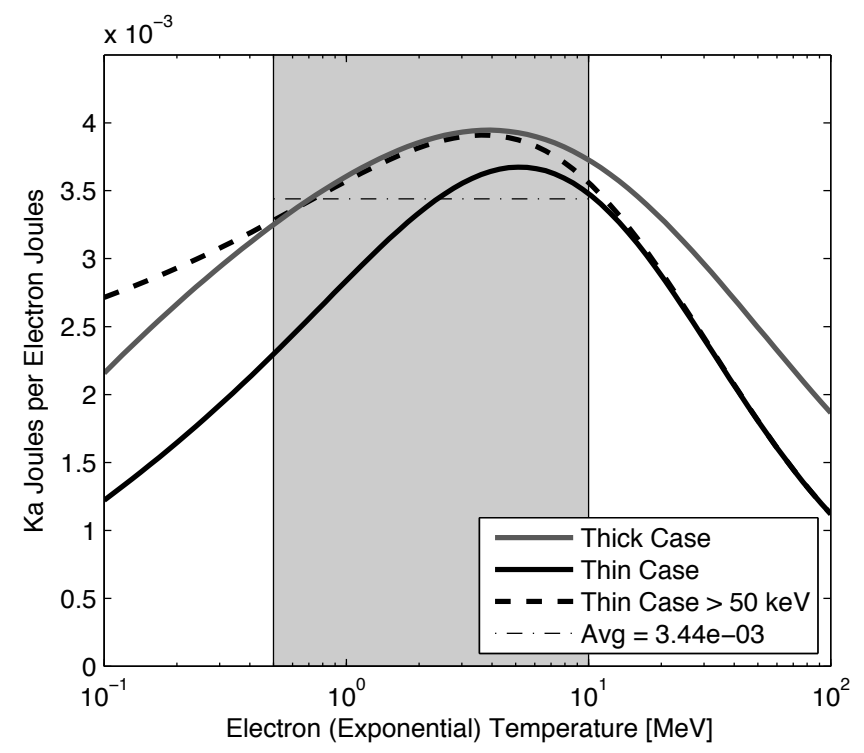

Figure 6.4: K $\alpha$ generation efficiency by electron impact ionization.

We can make a rudimentary analytical model to determine the amount of electrons that are trapped within the wire by estimating the electric potential along the edges of the wire. 


\subsection{Electrostatic Charging}

Large amounts of electrons are accelerated into the target by the laser. At the begining of the injection, the electrons will simply exit the target ballistically, if they have enough momentum to overcome the collisional stopping power of the target. However, as each succesive electron leaves the target, a charge builds up on the target and prevents electrons from leaving if their kinetic energy is not great enough to over come the potential. In time, the ions will accelerate outward as they are pulled out by these fields, however the time scales for ion motion are much slower than that of electrons due to their higher mass. The dynamics of this charging are quite complicated (as will be discussed in Chapter 7), yet it is useful look at this problem analytically to get an idea of the way that charge develops across the target. To do this, the target is modeled as a wire capacitor surrounded by a spherical conductor at infinity.

\subsubsection{Wire charging}

If we assume that the target is a capacitor, where $V$ is the voltage of the capacitor, $Q_{\text {loss }}$ is the escaped charge and $C$ is the capacitance, then

$$
V=\frac{Q_{\text {loss }}}{C}
$$

The escaped charge is the charge of an electron $q_{e}$ times the number of escaped electrons $N_{\text {loss }}$. To calculate the number of escaped electrons a zerodimensional method is used where all electrons with energies $\mathcal{E}$ great enough to overcome the potential $V$ will leave and those with lower energies will be trapped.

We assume that the electrons have an exponential energy distribution function (i.e. $\frac{d N}{d \mathcal{E}}=\frac{N}{T} e^{-\mathcal{E} / T}$ ), with slope temperature $T$ and total number $N$. The escaping electrons are determined by integrating the distribution function from the minimum energy to escape (i.e. $\mathcal{E}=q_{e} V$ ) to infinity.

$$
N_{\text {loss }}=\frac{N}{T} \int_{q_{e} V}^{\infty} e^{-E / T} d E=N e^{-q_{e} V / T}
$$


Using the capacitor at infinity model from (6.1), the above equation determines the voltage of the target.

$$
V=\frac{1}{C} q_{e} N e^{-q_{e} V / T}
$$

This equation is transcendental, but it can be solved using the Lambert $\mathrm{W}$ function, which solves the equation $z=\mathrm{W}(z) e^{\mathrm{W}(z)}$. So we can manipulate the previous equation to get it into the correct form.

$$
\frac{q_{e} V}{T} e^{q_{e} V / T}=\frac{q_{e}^{2} N}{C T}
$$

Where we can see that $\mathrm{W}(z)=q_{e} V / T$ and $z=q_{e}^{2} N / C T$. Combining these two equations we get the solution for $V$, which shows the dependence of voltage on the number, $N$ and temperature, $T$, of injected electrons. We reformulate this equation in terms of the total energy of injected electrons, $E_{e}=N T$. This equation shows that the charging of the wire is dependent on both the slope temperature of the accelerated electrons as well as their total energy.

$$
q_{e} V=T \times \mathrm{W}\left(\frac{q_{e}^{2} N}{C T}\right)=T \times \mathrm{W}\left(\frac{q_{e}^{2} E_{e}}{C T^{2}}\right)
$$

\subsubsection{Energy Fraction in Wire}

Now we have an equation to determine the voltage of the wire, $V$, and thus the minimum energy needed to escape the wire, $q_{e} V$. We can then determine the fraction of energy trapped within the wire. We determine the amount of energy remaining in the wire, $E_{\text {wire }}$ through integrating the energy distribution function $\mathcal{E} \times d N / d \mathcal{E}$ from zero to $q_{e} V$, where we have already solved for $q_{e} V$.

$$
E_{\text {wire }}=\frac{N}{T} \int_{0}^{q_{e} V} \mathcal{E} \times e^{-\mathcal{E} / T} d \mathcal{E}=N T\left[1-e^{-q_{e} V / T}\left(\frac{q_{e} V}{T}+1\right)\right]
$$

The percentage of energy in the wire is $P_{\text {wire }}=E_{\text {wire }} / E_{e}$. Substituting in the value of $q_{e} V$ from (6.5).

$$
P_{\text {wire }}=1-e^{-\mathrm{W}\left(\frac{E_{e} q_{e}^{2}}{C T^{2}}\right)}\left[\mathrm{W}\left(\frac{E_{e} q_{e}^{2}}{C T^{2}}\right)+1\right]
$$


Interestingly the fraction of energy trapped within the wire is only dependent on the parameter $\frac{E_{e} q_{e}^{2}}{C T^{2}}$. For a given target, $q_{e}$ and $C$ will be fixed, which means that the energy fraction trapped with the target is a function of the total electron energy $E_{e}$ divided by the slope temperature squared $T^{2}$. We plot the energy fraction versus this parameter in Figure 6.5. Here we can clearly identify three different regimes. First, when there is only a small amount of energy input or high slope temperature, then the target behaves in a no-field manner and no electrons are trapped within the wire due to fields. In the case where we have injected a large amount of energy or low slope temperature, then the field are very strong and almost all of the electron energy is trapped within the wire. Connecting these two regimes is a transitional regime where the amount of energy trapped within the wire is strongly dependent on the amount of energy in the electrons and their temperature.

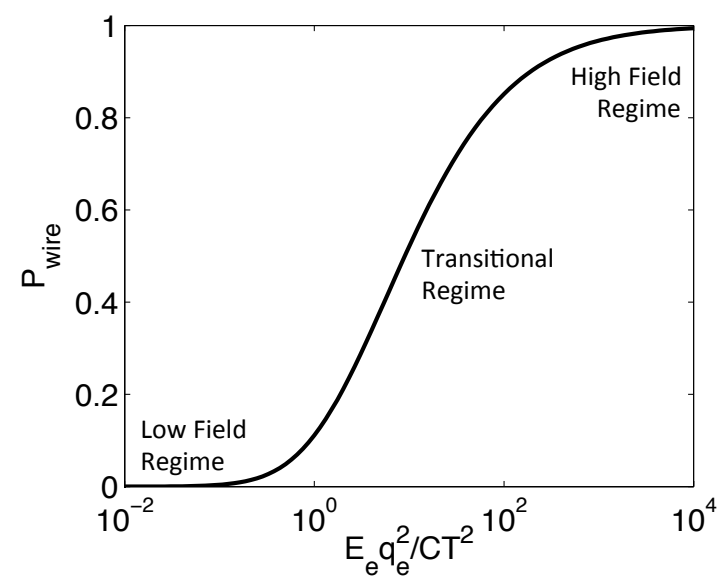

Figure 6.5: Energy fraction trapped in wire for a given $\frac{E_{e} q_{e}^{2}}{C T^{2}}$.

\subsubsection{Capacitance}

To get an idea of which regime applies to our experiments we must determine the capacitance of the wire. The capacitance of a finite length, finite radius wire is derived in a paper by Jackson, ${ }^{[89]}$ where $R$ is the radius of the wire, $L$ is the length of the wire and $\Lambda=2 \ln (L / R)$. In this equation $O\left(1 / \Lambda^{3}\right)$ indicates that 
the value is correct to order $1 / \Lambda^{3}$.

$$
\begin{aligned}
C & =4 \pi \epsilon_{0} \frac{L}{\Lambda}\left\{1+\frac{0.6137}{\Lambda}+\frac{1.0868}{\Lambda^{2}}+O\left(1 / \Lambda^{3}\right)\right\} \\
& =2.10 \times 10^{-14} \text { farads at } L=1.5 \mathrm{~mm}, R=20 \mu \mathrm{m}
\end{aligned}
$$

\subsubsection{Expected Values}

We have now developed some estimates of how the wire charges and we have calculated the expected value of capacitance. So we can now get an idea of how the wire should behave. From our calculated capacitance, we have

$$
\frac{E_{e} q_{e}^{2}}{C T^{2}}=47.7 \times \frac{E_{e, \mathrm{~J}}}{T_{\mathrm{hot}, \mathrm{MeV}}^{2}}
$$

In our experiments we expect to have total electron energies from 1 to 20 $\mathrm{J}$ and electron temperatures from from 0.1 to $5 \mathrm{MeV}$. This gives values of $\frac{E_{e} q_{e}^{2}}{C T^{2}}$ from $10^{0}$ to $10^{4}$. As is seen from the graph in Figure 6.5, these values are within the transitional regime. This means that the amount of energy that is trapped within the wire and the dynamics of the system are highly dependent on the amount of energy and temperature of the electrons. This observation illustrates the need to model the cone-wire system including the full physics of electric charge build up along the wire. This shows the importance of running simulations that model the generation of electric and magnetic fields self-consistently with Maxwell's equations.

\subsection{Resistive Stopping}

In addition to the electric potential build up along the wire, another collective effect that can potentially occur within the cone-wire system is called resistive stopping. This occurs when a substantially high current of electrons passes through a dense material. As shown in the physics section, this causes a return current to be drawn that will match the forward current density. Since this return current is low energy, it is highly collisional and thus an ohmic electric field develops. The strength of the electric fields can be given by a simple Ohm's law $\mathbb{E}=\eta j$, 
where $\eta$ is the resistivity of the material, $\mathbb{E}$ is the electric field and $j$ is the current density of the background electrons. For a beam of electrons with average kinetic energy $T$ and total energy $E$, which are evenly distributed over the wire with radius $R=20 \mu \mathrm{m}$ and for a time period that is represent by a gaussian function of full-width-at-half-maximum $\tau_{\mathrm{FW}}=650 \mathrm{fs}$, we can calculate the peak current, $j_{\text {peak }}$.

$$
j_{\text {peak }}=q_{e} \frac{E}{T} \frac{1}{\pi R^{2}} \frac{1}{\tau_{\mathrm{FW}}} \sqrt{\frac{4 \ln 2}{\pi}}
$$

For a beam with $10 \mathrm{~J}$ of electron energy and a mean kinetic energy of $1 \mathrm{MeV}$ the peak current density is $10^{12} \mathrm{Amps} / \mathrm{cm}^{2}$. From the physics section, a copper plasma at $1 \mathrm{eV}$ has a resistivity of around $10^{-7} \Omega \mathrm{m}$ and a peak resistivity of around $10^{-6} \Omega \mathrm{m}$. These values give peak electric fields of 1 and $10 \mathrm{kV} / \mu \mathrm{m}$, respectively. Such field strengths are comparable to the stopping powers of electrons, as shown in Figure 2.11 of Section 2.4.5. Therefore depending on the resistivity, the electric field strength, may or may not, play a large role in the development of the system. Again, we are in a regime, where treating the growth or electro-magnetic fields is very important, and simulations including this are necessary. 


\section{Transport Simulations}

Now that we have a qualitative understanding of the relevant physics for the cone-wires, we move on to a more quantitative treatment where we model the electron transport including the self-consistent generation of electric and magnetic fields. To do this work, we run hybrid-PIC simulations using the code LSP. The general working of PIC simulations and LSP is described in Chapter 4; here we describe the specific setup for our runs. A depiction of the geometry is shown in Figure 7.1. This model includes the majority of the cone-wire target and in the same dimensions. The Au cone extends backwards $300 \mu \mathrm{m}$ from the tip and the $1.5 \mathrm{~mm} \mathrm{Cu}$ wire is completely included, both were modeled at solid density.

\subsection{Simulation Setup}

Both the $\mathrm{Au}$ cone and the $\mathrm{Cu}$ wire are included in this simulation and are modeled as fluid particles. These ions and electrons interact with each other through the equation-of-state provided by PROPACEOS ${ }^{[85,86]}$ and temperaturedependent collision frequencies. ${ }^{[64,65]}$ Vacuum boundaries of $1 \mathrm{~mm}$ were extended from the edges of the target to allow particle trajectories to be fully realized and the full field evolution to take place. A single cell of protons at $2 \times 10^{20} \mathrm{~cm}^{-3}$ density was distributed along the edges of the cone and wire. These represent the contaminant layer found on most targets and are important because protons can accelerate more quickly than heavy ions and thus change how fields develop with time. The cell size used in this simulation is $1 \mu \mathrm{m}$ in the solid density plasma and

cells increase in size in the vacuum to speed up the simulation. The time step is 0.5 times the Courant limit, that is particles traveling at the speed of light would 
need 2 timesteps to cross the smallest cell.

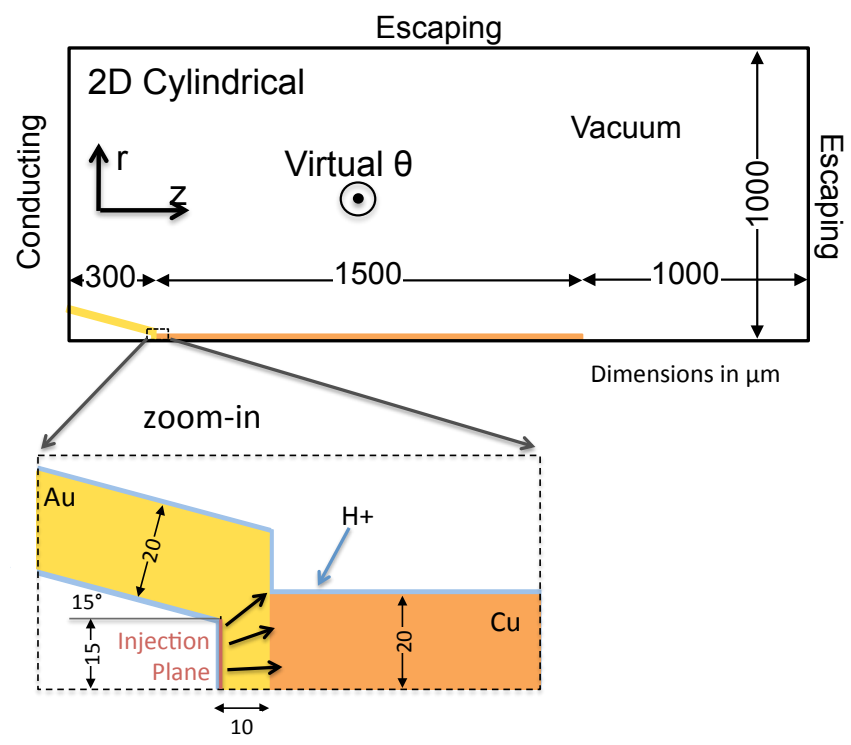

Figure 7.1: Setup of the transport simuations in LSP.

In these transport simulations, the laser interaction is not modeled, instead electrons are injected at the At cone-tip. The electron energy distribution was the summation of one or two decaying exponentials, $f(E)=\exp (-E / T)$ with kinetic energy $\mathcal{E}$ and slope temperature $T$. The electrons were given a gaussiandistributed momentum, as a function of solid angle. They were injected at the inner cone-tip of the target with a gaussian distribution in space along the radius of the tip that matched the spatial distribution of the laser. The injected profile in time was a gaussian function matched to the laser's profile. The laser temporal and spatial functions were not precisely the same on Trident and Titan, however they are very similar, as described in Chapter 3. Thus, to not complicate things, the same spatial and temporal distributions were used when trying to match the data from both experiments. The electrons undergo collisions treated through a test particle model ${ }^{[33]}$ with the addition of radiative stopping ${ }^{[9]}$ and $\mathrm{K}_{\alpha}$ generation cross sections. ${ }^{[71]}$

The purpose of these simulations was to diagnose the falloff and coupling efficiency of given electron spectra. We need to run many simulations and vary the parameters of injection in order to understand these dependencies. However, in 
order to fully understand the physics that underlie these dependencies, we first describe a single simulation and characterize the transport of electrons. As the main physics of the transport does not change, this description will be representative of all of the simulations that were run.

\subsection{Understanding the Transport of Electrons within the wire}

The particular simulation that we look at had a total injected electron energy of 3.5 J. The energy spectrum was a two temperature exponential function, with a $0.5 \mathrm{MeV}$ cold slope and a $7.0 \mathrm{MeV}$ hot slope, where $70 \%$ of the energy was contained in the hot component. We differentiate these two electron populations as the COLDS and the HOTS. Other than their slope temperatures, these electrons are identical. The electrons had a $45^{\circ}$ gaussian angular distribution.

To understand the evolution of electrons we look at three snap-shots in time and describe the trajectories of electrons and the interaction/creation of electric and magnetic fields.

\subsubsection{Initial Time: Electrons Initial Injection}

The first snapshot is at $2.5 \mathrm{ps}$, in Figure 7.2, after most of the electrons have been injected into the simulation (from the lefts side). Already, there is a large difference in the behavior of the HOTS and COLDS. In both populations, there is a bunch of electrons that are traveling ahead of the others. They are the first electrons injected so they travel ballistically (i.e. unimpeded by fields), because the charge within the system is small enough that the fields have not yet gotten large. However, electrons traveling later in time already begun to experience confining electric fields that trap them within the wire. As a consequence, later electrons cannot escape the wire as easily. Notice that the COLDS are much more concentrated within the wire, as they do not have high enough kinetic energy to escape. The triangular shape at the start of the wire is due to the motion of 

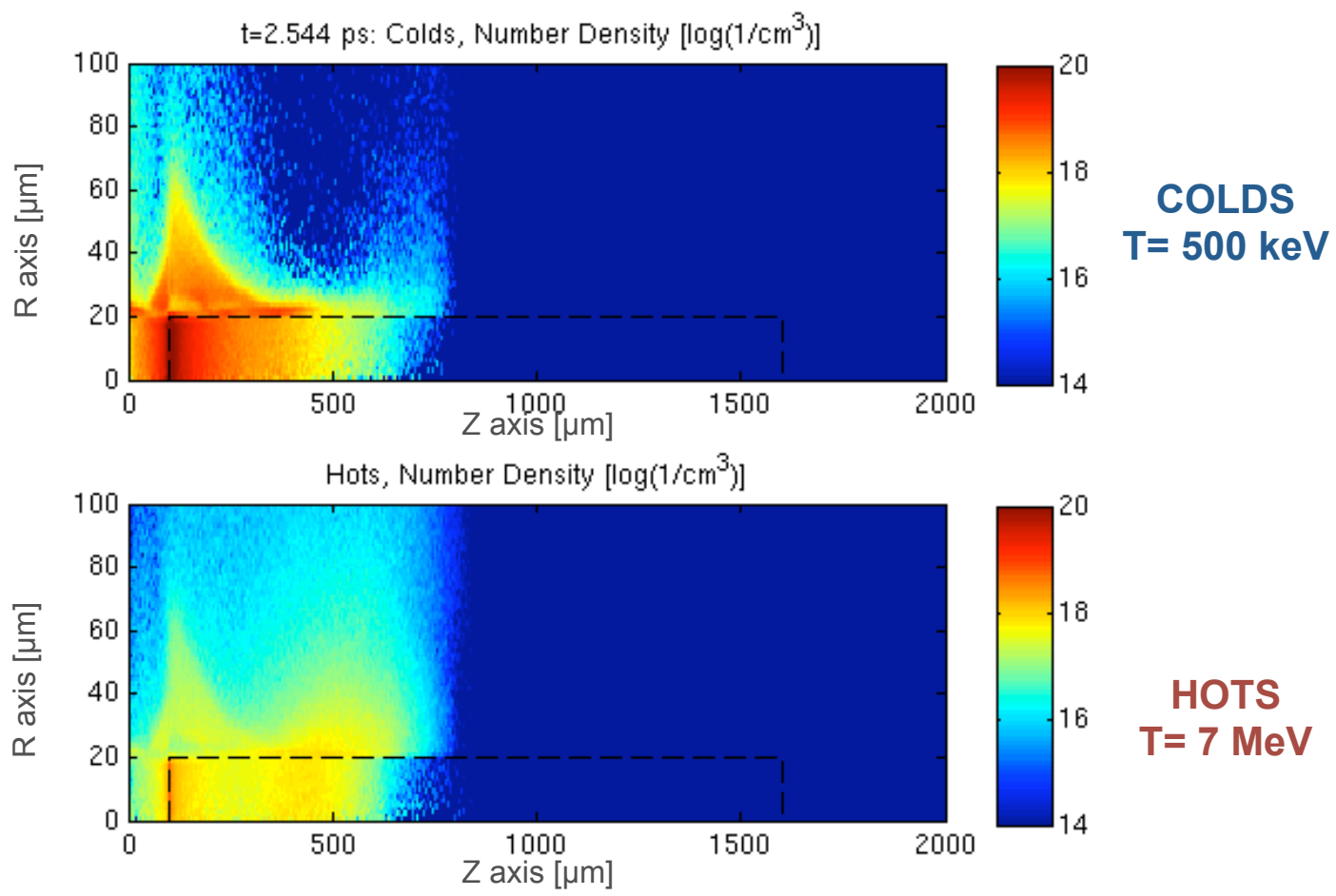

Figure 7.2: Injected electron density plots during the first snap-shot of electron transport simualation at $2.5 \mathrm{ps}$.

protons. As protons are pulled from the wire surface by strong electric fields, they allow electrons to travel with them in a quasi-neutral bunch.

\subsubsection{Middle Time: Electrons Surf Along the Wire}

The next snapshot is taken at $5.6 \mathrm{ps}$, shown in Figure 7.3. At this point, many of the electrons have stopped in the beginning of the wire. These are the electrons that create the initial falloff and for our purposes of understanding electron spectra these are the most important. However, in understanding the creation mechanism for the bump at the end of the wire, we must look to the population of electrons that appear to be surfing along the edge of the wire.

The surfing electrons are most noticeable in the COLD component at about $1000 \mu \mathrm{m}$ in the z-direction and along the edge of the wire. In order to understand the forces that keep these electrons along the wire, we take a lineout of the electric 


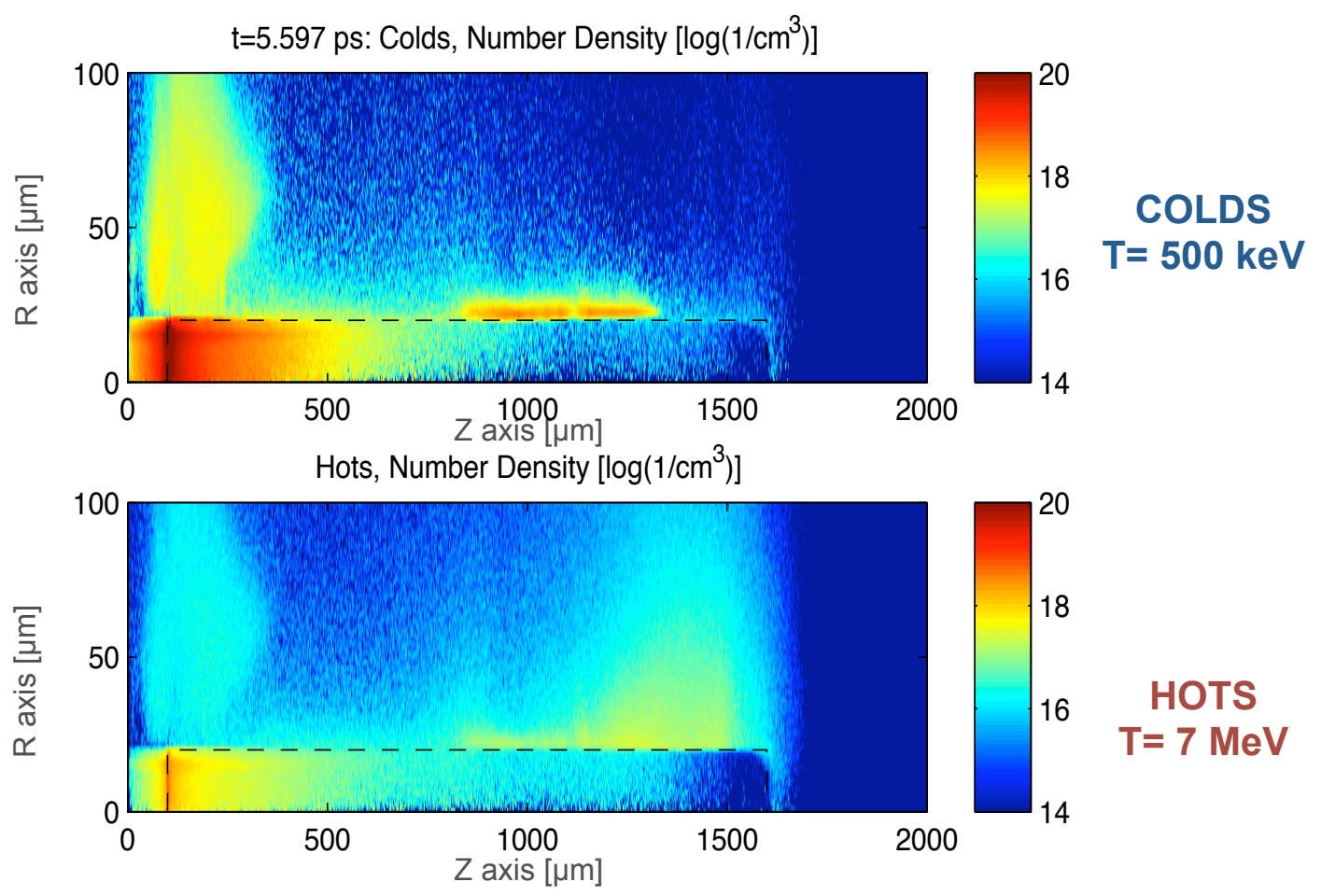

Figure 7.3: Injected electron density plots during the second snap-shot of electron transport simualation at $5.6 \mathrm{ps}$.

and magnetic fields at the point where the electrons are surfing. Instead of simply plotting the electric and magnetic fields, we plot the forces that would be exerted by these fields in the radial direction by electrons traveling at the speed of light (as they are fully relativistic) in the positive $z$-direction. These forces are created by the radial electric field and a azimuthal magnetic field.

We see from the lineouts of these force in Figure 7.4, that the electric and magnetic forces are comparable in strength. Also, they are in opposition. The radial electric field is pushing electrons into the wire, since it is created by electrons that leave the wire and cause it to charge up. However, the azimuthal magnetic field is actually pushing electrons away from the wire. While we understand the mechanism to create the electric field, the magnetic field creation is much more puzzling.

How is this magnetic field created? To understand this we take the total 

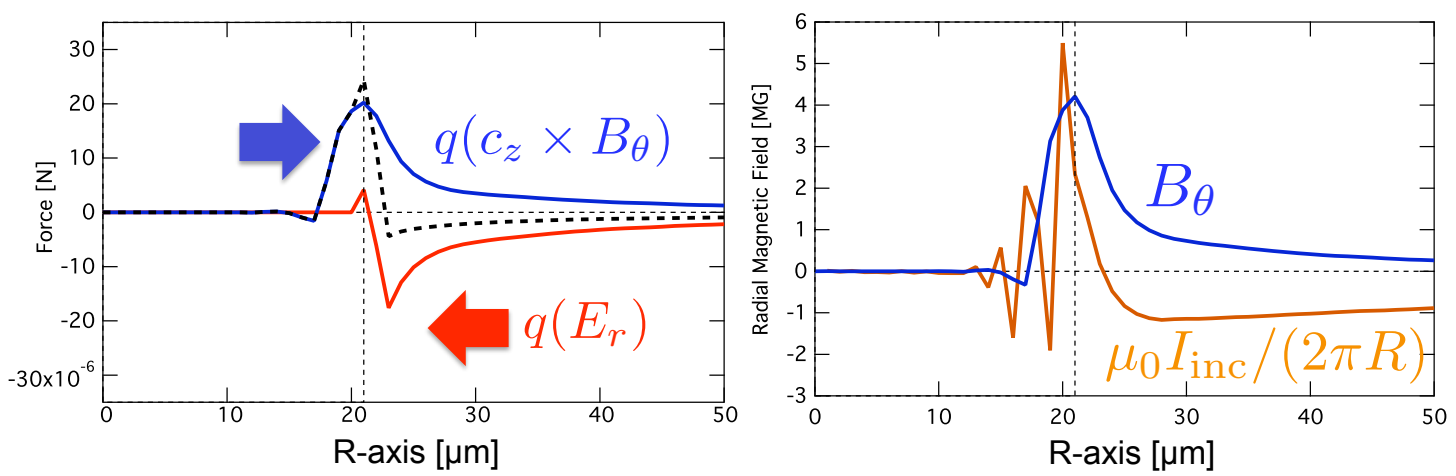

Figure 7.4: Radial lineouts of magnetic and electric forces (left). Measured and inferred magnetic fields (right) during the second snap-shot at 5.6 ps taken near the surfing electrons at $1000 \mu \mathrm{m}$.

encircled current along the radial axis. Using Biot-Savart's law we can show a large portion of the magnetic field is generated by this current. Interestingly, this current is flowing in the direction toward the cone and away from the rear end of the wire. This is the opposite to the direction that fast electrons are traveling. Thus the magnetic field generated is opposite of what we would expect if the electrons were injected into vacuum, where the magnetic field generated by the electrons would tend to pinch the beam rather than expand it. So what is the mechanism for this current generation?

The mechanism is as follows: when electrons escape the wire, they create a large charge imbalance that draws a current as illustrated in Figure 7.5. This causes an electron current to be drawn from within the wire. Since the charge imbalance is very large, it draws a very large current. This current cannot escape the wire, thus even though the net current across the entire radius (including vacuum) would be forward traveling, the net current within the wire is backward going. Since the magnetic field is created by encircled current, the total magnetic field is pointing in a direction so to expell the forward traveling injected electrons. Thus the radial electric field and the azimuthal magnetic field are intrinsically related, because they are both stem from the charge differential created by escaping electrons. 
(a)

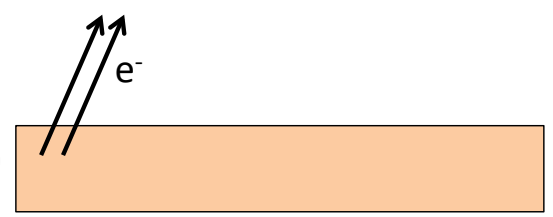

(b)

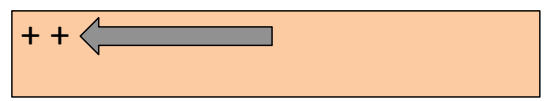

(c)

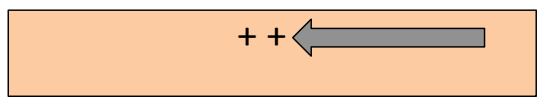

(d)

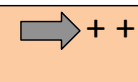

Figure 7.5: Cartoon of the generation of the current thorough charge imbalance. In (a) fast electrons leave the wire creating an electric field due to the charge difference. This draws a current (b) through the electric field, and this propagates through the wire (c). When the leading edge of the current reaches the end of the wire (d) it can no longer propagate, and creates a backward flowing current. This process will continue until the wire equilibriates to a constant potential.

\subsubsection{Final Time: Electrons Pushed into Wire}

The next Figure 7.6 shows the snap-shot at 7.1 ps in time where the surfing electrons are being pushed back into the wire. At this point, there are a few factors that stop the charge-imbalance current from flowing or that can potentially reverse its direction. First, the leading edge of the current reaches the end of the wire. Since there is only vacuum at the end of the wire, there is no material to keep drawing current from. Thus this current can no longer be sustained and it reverses. The other effect is that the hottest electrons (i.e. not the surfing electrons) reach the end of the wire. As they leave the wire another charge imbalance is created that draws current to equilibrate. Both of these effects cause the total encircled current within the wire to flip direction. Thus the magnetic field also flips direction and the force that previously kept the electrons from falling in the wire, now pushes them into the wire. As the field information can only propagate at the speed of light, it reaches the electrons at different times in the bunch. This can be seen in the Figure 7.6 where electrons after $1250 \mu \mathrm{m}$ are being pushed into the wire, while those before are still surfing along the edge. This effect of surfing electrons and their entry into the wire is the cause of the bump at the end of the wire as seen in the $\mathrm{K}_{\alpha}$ emission profiles. 


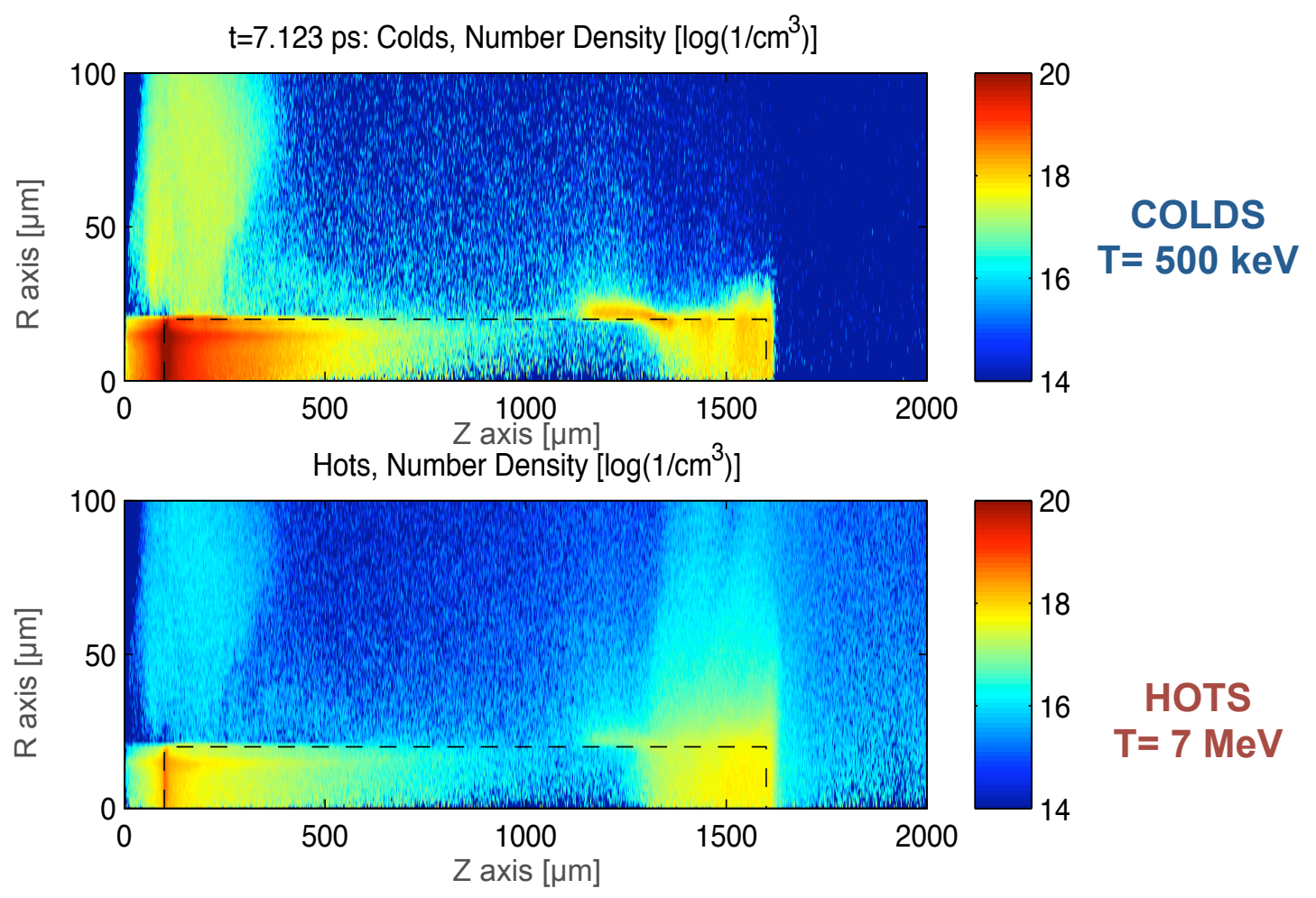

Figure 7.6: Injected electron density plots during the third snap-shot of electron transport simualation at $7.1 \mathrm{ps}$.

The field lineouts in Figure 7.7 are taken along the wire at $1400 \mu \mathrm{m}$ where electrons are now getting pushed into the wire. One can see that both the electric and magnetic fields are now pushing forward-going electrons into the wire. Again, the majority of this magnetic field is caused by the total electron current, which is now headed in the opposite direction as previously.

Figure 7.8 shows the simulated $\mathrm{K}_{\alpha}$ emission profile, where we see that all of the important portions of the wire are reproduced and now we have a better idea of which electrons produced them. The initial falloff is produced mostly by the colder electrons. The middle flat portion of the wire is produced by hotter electrons. Finally, the bump at the end of the wire is produced by the colder electrons, but it is these electrons that are surfing along the edge of the wire. The electric and magnetic fields that allow these electrons to surf are produced by the electrical charging of the wire and this is highly dependent on the energy of the 

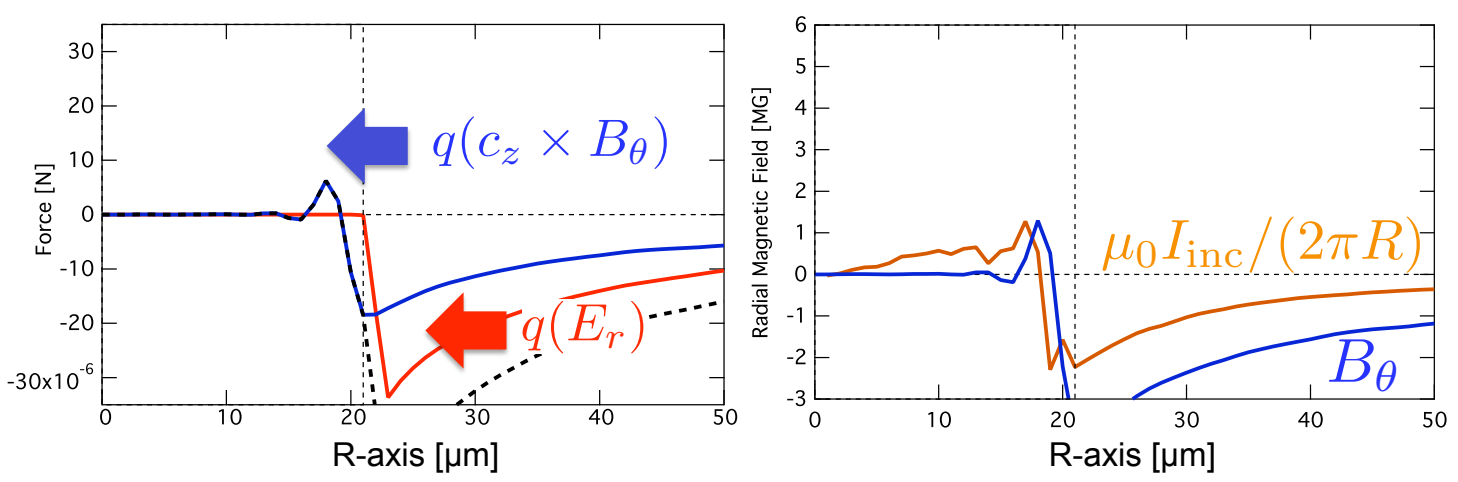

Figure 7.7: Radial lineouts of magnetic and electric forces (left). Measured and inferred magnetic fields (right) during the third snap-shot at 7.1 ps taken at 1400 $\mu \mathrm{m}$ where electrons are being pushed into the wire.

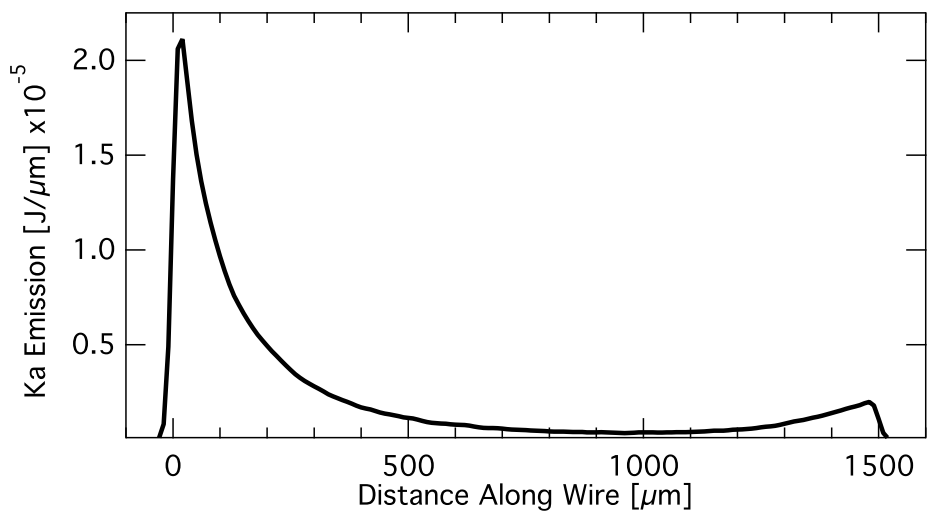

Figure 7.8: Simulation of the $\mathrm{K}_{\alpha}$ emission profile using an injected electron distribution that reproduces the many features of the cone-wire experimental data.

hottest electrons. Thus it is difficult to use the bump as a diagnostic for electron spectra since it is intertwined with so many different aspects of the system.

\subsection{Partition of Energy in the Simulation}

One of the most simple ways to look at the differences in the electron transport is to look at the energy partitioned in the simulation. To illustrate this we show the energy partition as a function of time in Figure 7.9 for two single exponential distributions of 0.75 and $2.0 \mathrm{MeV}$ slope temperatures both with a 
total of $10 \mathrm{~J}$ of injected energy. We calculate the amount of energy in the fields, into the protons, into the $\mathrm{Au} / \mathrm{Cu}$ ions and electrons, and the energy of the injected electrons that leaves the simulations through the borders (i.e. escapes). As a note, the reason that the total energy does not add up to $10 \mathrm{~J}$ (as was injected) is due to the fact that LSP does not easily output the ionization energy that goes into the EOS and this energy would make up the majority of the missing energy.

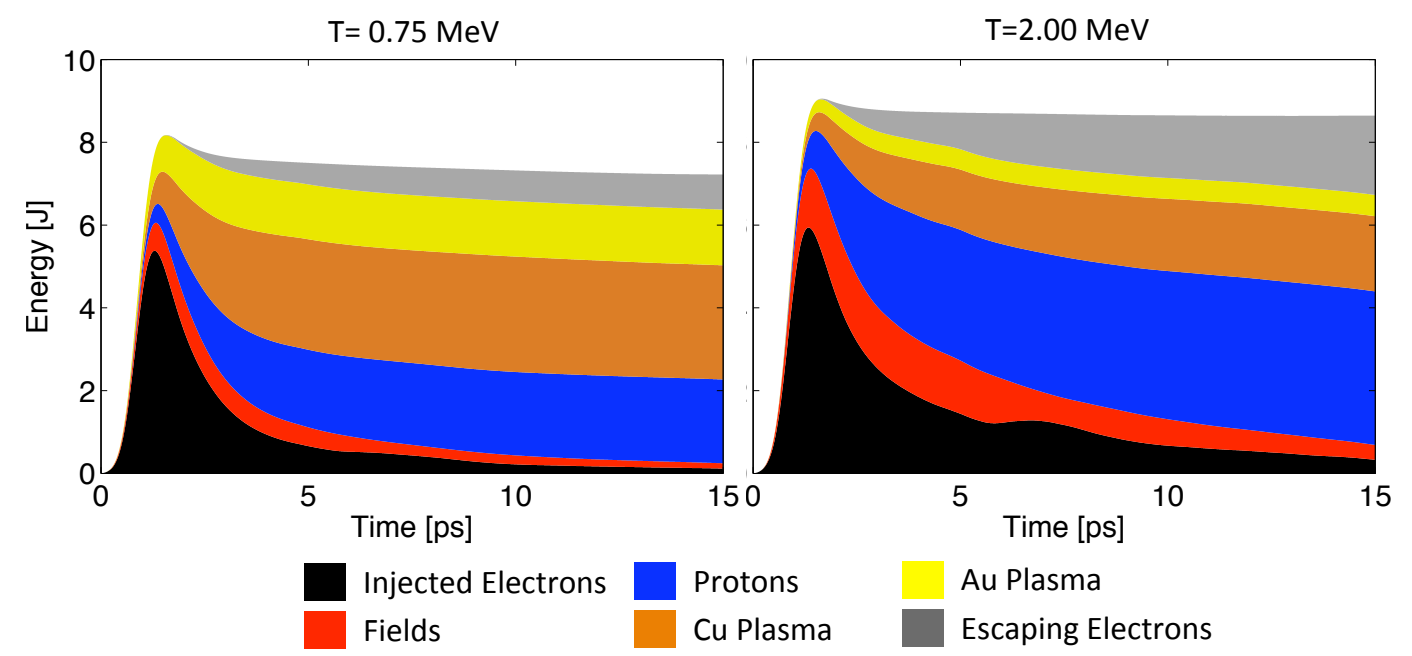

Figure 7.9: Partition of energy in the simulation as a function of time for single temperatures of 0.75 (left) and 2.0 (right) $\mathrm{MeV}$ slope temperatures.

We can see that the case of hotter electron slope temperature that much more energy goes into energy that escapes the $\mathrm{Au} / \mathrm{Cu}$ target (i.e. fields, protons, escaping). As we explained in the previous section, this is due to the fact that hotter electrons have more kinetic energy to escape the electric fields that up on the target. On the other hand, the colder temperature case has more energy in the $\mathrm{Au} / \mathrm{Cu}$ plasmas, since these electrons are less likely to escape.

In Section 6.3.2, we derived an analytical solution to an exponential electron spectrum that was electrically confined to a target based on the charging induced by the escaping electrons and a given capacitance of the target. We found in Section 6.3.2 that this was a function of the total injected energy $E$ divided by the electron slope temperature $T$ squared. In Figure 7.10, we show the conversion efficiency of $\mathrm{K}_{\alpha}$ plotted against this $E / T^{2}$ for a variety of injected electron energies. 
Notice that the this shows a similar dependence as the Lambert $W$ function that was derived previously. The main difference from this solution is that at high values of $E / T^{2}$ that the conversion efficiency goes to zero. This is because the high values of $E / T^{2}$ correspond to low value of $T$, which are often stopped within the $\mathrm{Au}$ cone. This energy mechanism was not included in our analytical solution.

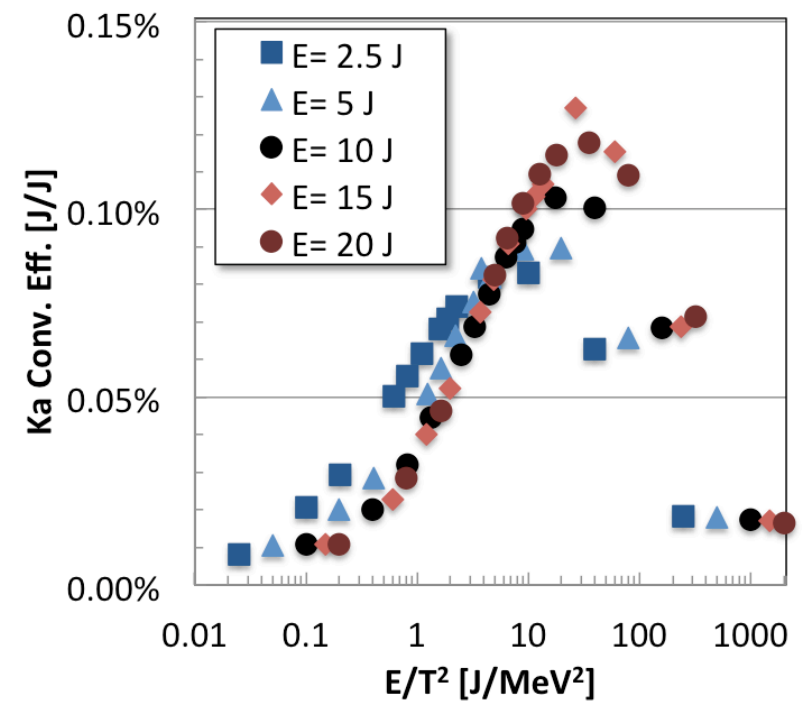

Figure 7.10: Conversion efficiency into $\mathrm{K}_{\alpha}$ photon energy as a function of $E / T^{2}$.

From the two previous figures, we see that electric fields play a major role in the transport of electrons through the target. In fact, in both simulations, nearly $50 \%$ of the energy goes into electrons escaping the target and the fields/protons. For this reason, modeling the fields correctly is very important to get the correct amount of energy coupling into the wire and to correctly infer the electron spectrum. There were two main effects that we made sure to include to model the electric fields accurately. The first one was setting up the simulation in a 2D cylindrical geometry, whereas, in some previous work simulations of wire targets were modeled in a 2D Cartesian geometry. ${ }^{[49,50]}$ Electro-static fields falloff like $1 / r^{2}$ in a cylindrical geometry and $1 / r$ in a slab geometry. Thus to reproduce the natural cylindrical symmetry of the wires, we must use a 2D cylindrical geometry.

Another important factor in modeling fields is the inclusion of a proton layer. In our simulations, we have included a 1 cell thick and $2 \times 10^{20} \mathrm{~cm}^{-3}$ 


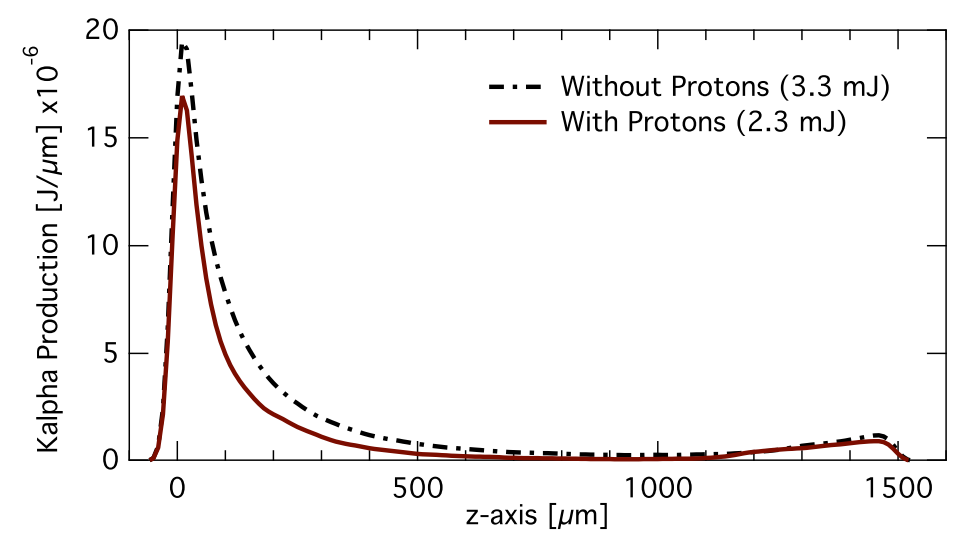

Figure 7.11: Profile of $\mathrm{K}_{\alpha}$ emission with and without a proton layer.

density proton layer to reproduce the contaminant layer found on the targets used in laser experiments. This layer is important to include because ionized protons have a higher charge-to-mass ratio than the $\mathrm{Au} / \mathrm{Cu}$ ions. Thus the protons are accelerated much more easily in the electric field and are able to dissipate some of this field. Because these protons move relatively quickly, they can dissipate the electric fields on timescales that are within the transport time of the electrons. Figure 7.11 shows a simulation run with protons compared to one without protons. Less electron energy is trapped within the wire when protons are included as the protons dissipate the electric fields and allow more electrons to escape. The total $\mathrm{K}_{\alpha}$ emission drops about $30 \%$ when a proton layer is added to the simulation. This shows the importance of including the protons layer to attempt to accurately model the field development in the system.

\section{4 $\mathbf{K}_{\alpha}$ Production from Different Electron En- ergy Bins}

To quantitatively assess the $\mathrm{K}_{\alpha} \mathrm{x}$-ray production, we implemented a technique to keep track of the initial energies of electrons as they emitted $\mathrm{K}_{\alpha} \mathrm{x}$-rays. This allowed us to assess which energy electrons were responsible for specific portions of the $\mathrm{K}_{\alpha}$ profile and to compare this with our simple understanding developed in the previous chapter. 


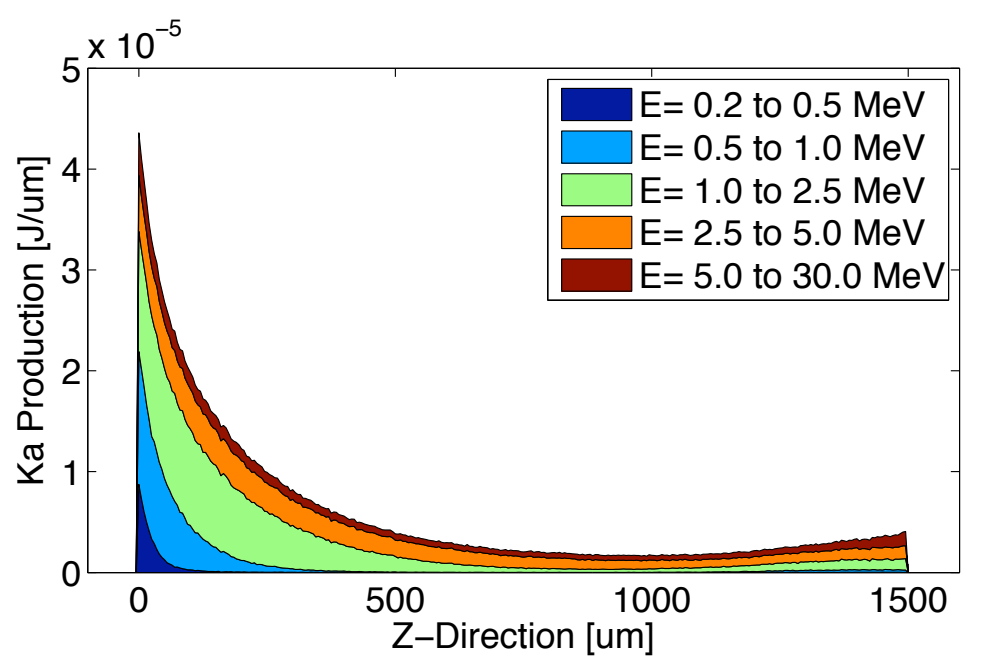

Figure 7.12: Production of $\mathrm{K}_{\alpha}$ binned for the injected energies.

This $\mathrm{K}_{\alpha}$ profile is shown in Figure 7.12, where the different colors represent different bins of initial electron energies. Notice that electrons below $2.5 \mathrm{MeV}$ falloff strongly in the initial portion of the wire, while electrons with higher energies have a relatively constant $\mathrm{K}_{\alpha}$ emission through out the entire distance of the wire. This is a verification of the simple arguments made in Chapter 6 to show that electrons above $2 \mathrm{MeV}$ lose energy slowly in a distance of $500 \mu \mathrm{m}$.

To show, which electrons are responsible for the total $K_{\alpha}$ emission, we plot the normalized cumulative amount of $\mathrm{K}_{\alpha}$ emitted as a function of energy in Figure 7.13. This figure shows that low energies of electrons below about $0.5 \mathrm{MeV}$ do not emit very many $\mathrm{K}_{\alpha}$ x-rays. As explained in Chapter 6 , this is due to the stopping of electrons in the cone-tip, which will stop electrons below $0.2 \mathrm{MeV}$ completely. Additionally, the electrons above $10 \mathrm{MeV}$ do not contribute much to $\mathrm{K}_{\alpha}$ production, because they have high enough energies so that they can escape the electric fields that build up within the wire. In fact, $95 \%$ of the $\mathrm{K}_{\alpha}$ emission comes from electrons with energies between 0.5 and $10 \mathrm{MeV}$. 


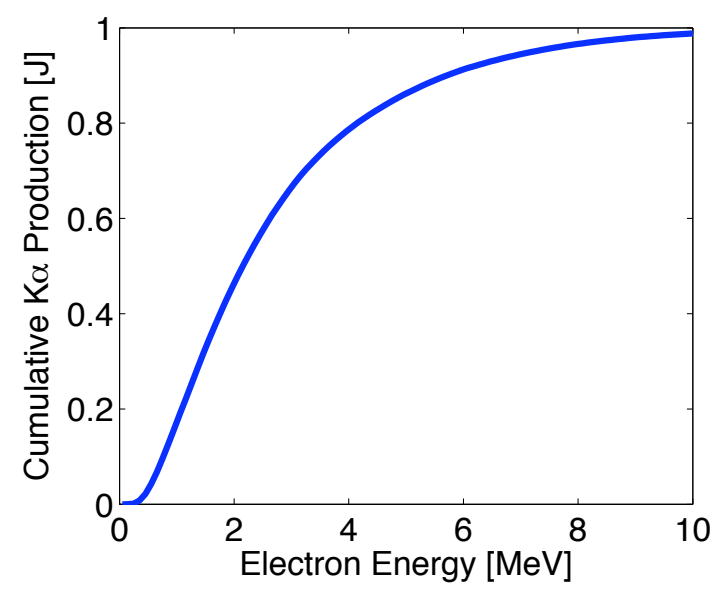

Figure 7.13: Cumulative $\mathrm{K}_{\alpha}$ production for given bins of injected electron energies, which show that $95 \%$ of the $\mathrm{K}_{\alpha}$ energy is produced by 0.5 to $10 \mathrm{MeV}$ electrons.

\subsection{Temperature of background electrons and $\mathbf{K}_{\alpha}$ Shifting}

In Section 3.4, we discussed the effect that temperature shifting and broadening play on our narrow-bandwidth crystal imager. This showed that if the background electron temperatures get very hot $\sim 100 \mathrm{eV}$ that the $\mathrm{K}_{\alpha}$ shifting may be large enough to change the observed $\mathrm{K}_{\alpha}$ x-ray image. To look into this effect, we have placed temperature probes at various locations within the wire and show how they develop with time in Figure 7.14. This Figure shows the temperatures rise to about $50 \mathrm{eV}$ in this simulation, where the peak temperature is at the front of the wire up to $10 \mu \mathrm{m}$ and then decays substantially within $100 \mu \mathrm{m}$. A $50 \mathrm{eV}$ temperature is not high enough to make a substantial change in the crystal reflectivity. However, injecting a larger amount of energy may much the maximum temperature up to near $100 \mathrm{eV}$ values. Thus we should be careful in what conclusions we draw from the first $100 \mu \mathrm{m}$ of the simulation, and we should note that this effect will be the most pronounced in the wire tip, which is while we have previously cautioned against relying heavily on the initial peak of the $K_{\alpha}$ profile. 


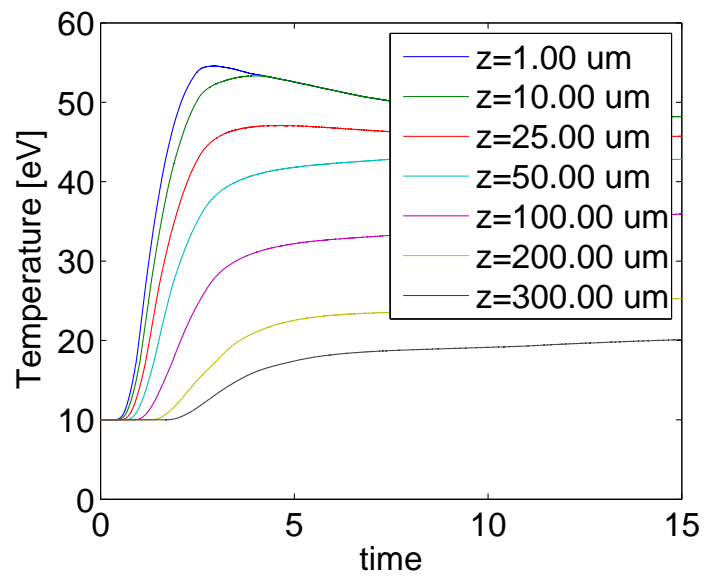

Figure 7.14: Background electron temperature along the wire at different depths as a function of time. Here is the wire is initialized at $10 \mathrm{eV}$.

\subsection{Single-Temperature Fits to the Data}

Now that we have an understanding of the transport of electrons through the wire, we want to use this information to translate the experimentally-measured falloff distance into electron spectra. To this goal, we ran multiple single-temperature exponential energy distributions of $f(\mathcal{E})=\exp (-\mathcal{E} / T)$, where $\mathcal{E}$ is the electron energy and $T$ is the slope temperature. We varied the temperature from 0.1 to 10 $\mathrm{MeV}$ and the total energy of the injected electrons from 2.5 to $15 \mathrm{~J}$. All of the injections had the same angular distribution with an angle $\Delta \theta=45^{\circ}$. The results of the simulations were quantified by measuring the integrated $K_{\alpha}$ and the $K_{\alpha}$ falloff distance, as done in the experiments. This created a matrix of the two variables and allowed interpolation between these values to match both the total $\mathrm{K}_{\alpha}$ and the falloff for each of the experimental points.

Both the total $\mathrm{K}_{\alpha}$ and the falloff must be matched concurrently, because there is correlation between them. This is due to the fact that the energy of electrons that are trapped within the wire is dependent on the fields and, hence, is non-linear. We show the falloff plotted against temperature for the $5 \times 10^{19} \mathrm{~W} / \mathrm{cm}^{2}$ case on Trident in Figure 7.15, where these values have already been interpolated to match the total $K_{\alpha}$. From this figure we see a strong dependence on falloff 
with electron temperature. This is what we expected due to the fact that hotter electrons travel further in the wire and thus produce a longer falloff.

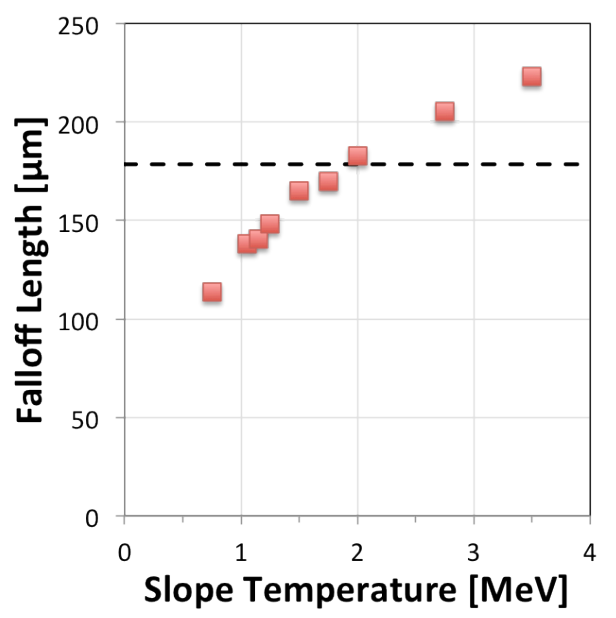

Figure 7.15: Falloff distance vs temperature of a single exponetial with a $\mathrm{K} \alpha$ energy interpolated to $7.09 \mathrm{~mJ}$ to match of the Trident laser at $5 \times 10^{19} \mathrm{~W} / \mathrm{cm}^{2}$. The experimental falloff length for this data point is shown as a dotted line.

We show a few points surrounding the best fit to the Trident data in Figure 7.16 to illustrate how well the simulations fit the experimental data. This figure gives an idea of the sensitivity of the fits as the 0.75 and $3.5 \mathrm{MeV}$ temperatures obviously do not fit well with the data.

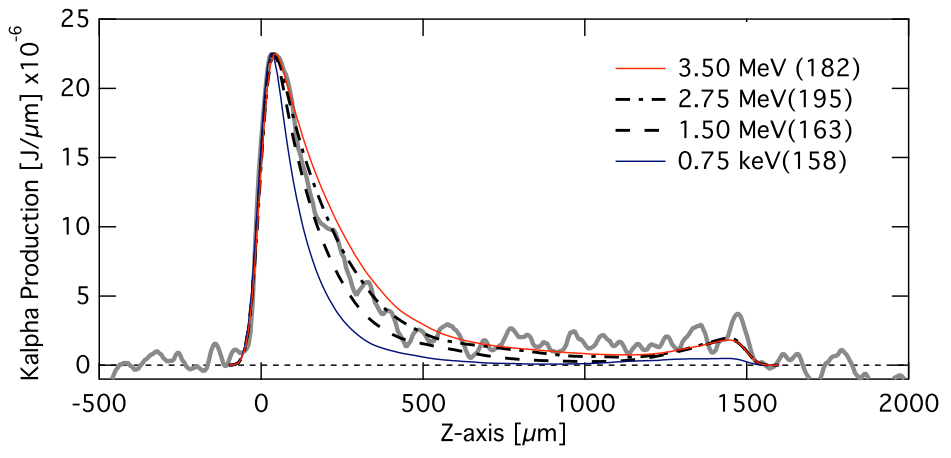

Figure 7.16: Simulation of the $\mathrm{K}_{\alpha}$ profile for different injected slope temperatures. Experimental data for the Trident laser at $5 \times 10^{19} \mathrm{~W} / \mathrm{cm}^{2}$ is plotted in gray.

The best fits to the highest intensity on Trident and Titan are shown in 
Figure 7.17. We can see that the simulations match very well with the data and that a 2 and $1.25 \mathrm{MeV}$ slope temperature match well with the Trident and Titan data, respectively. Notice that even with these single exponential fits that we can match all of the features of the wire, not only the inital falloff, but also the center flat region and the bump at the end of the wire. This seems relatively unexpected, because we have not tried to match the bump, we have only tried to match the initial falloff. The fact that the bump is matched so well makes us think that it is most likely independent of the electron spectrum and is therefore not really that useful in the electron energy spectrum determination.
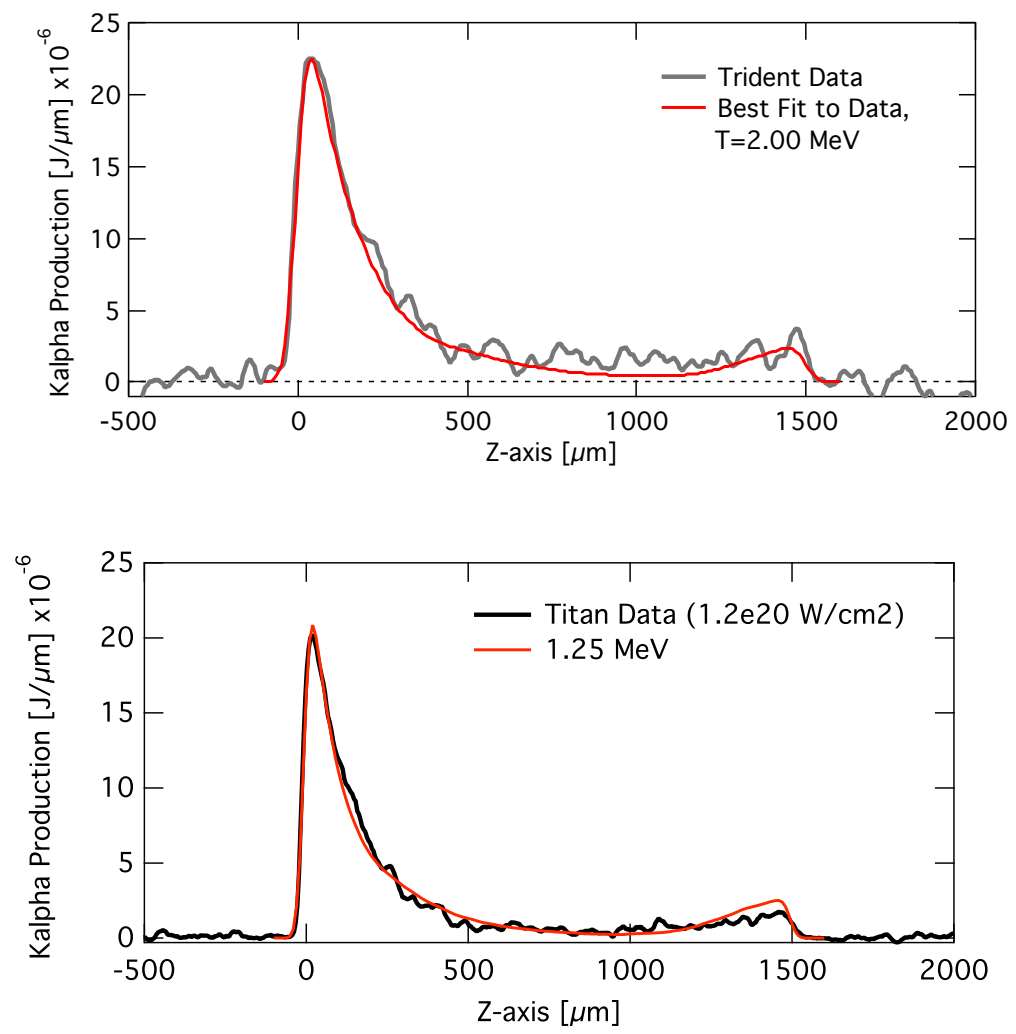

Figure 7.17: Experimental and simulated $\mathrm{K}_{\alpha}$ lineouts from from (top) the Trident laser at $5 \times 10^{19} \mathrm{~W} / \mathrm{cm}^{2}$ and (bottom) the Titan laser at $1 \times 10^{20} \mathrm{~W} / \mathrm{cm}^{2}$.

This is different than previous published work, ${ }^{[41]}$ where the bump was used to infer that there were two slope temperatures present in the electron spectrum. This previous work injected electrons with a Maxwell-Jüttner distribution, ${ }^{90,91]}$ which heavily weights the most energetic part of the spectrum and has few electrons 
at low energies ${ }^{1}$. Use of this type of energy distribution was most likely the reason that two temperatures were required. In contrast, Figure 7.17 clearly shows a that single exponential distribution function is capable of reproducing both the initial falloff and bump at the end of the wire.

Now that this interpolation technique is shown to be effective in matching the data, we can extend it to many points in our data and infer electron temperature. Figure 7.18 shows the inferred electron slope temperatures against laser intensity. This is similar to the plot of falloff distance versus intensity shown in Section 5.3, but now the falloff distance is replaced with inferred electron slope temperatures. The trend remains the same: hotter electron temperature with increasing laser intensity. This was assumed to be the case, however, it was not completely clear that the non-linear behavior of the system would not cause changes in these previously seen trends. Interestingly, the offset between the high-contrast data taken on Trident and the low-contrast data from Titan still shows that higher energies of electrons are observed in the wire at high-contrast.

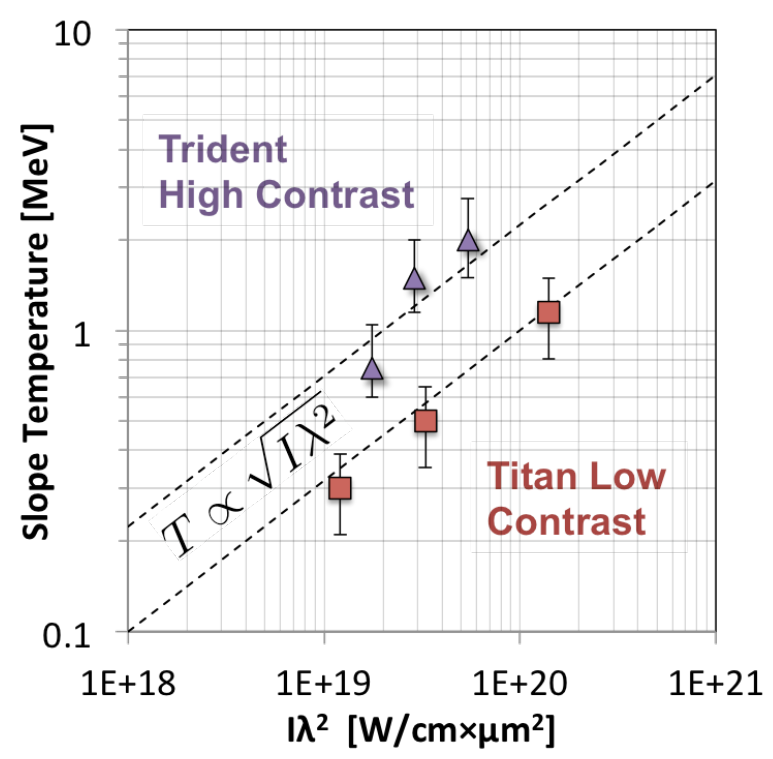

Figure 7.18: Slope temperatures of electrons inferred from single-temperature fits to the Trident and Titan data.

\footnotetext{
${ }^{1} \mathrm{~A}$ Maxwell-Jüttner distribution is a formulation of a gas in Boltzmann equilibrium including relativistic effects. The distribution is described as $f(\mathcal{E})=\gamma \sqrt{\gamma^{2}-1} \exp (-\mathcal{E} / T)$, where $\mathcal{E}$ is the electron kinetic energy, $\gamma$ is the relativistic factor and $T$ is the characteristic temperature.
} 
Figure 7.18 also shows dotted lines corresponding to temperature as a function of $\sqrt{I \lambda^{2}}$ as would be expected if the electron acceleration were due to the $\mathbf{j} \times \mathbf{B}$ mechanism as discussed in Section 2.3.3. It seems that our data is consistent with such a scaling. However, we must be careful about such a statement, since the preplasma plays a major role in the acceleration. In these points, the preplasma as well as the laser intensity may be chaning. To investigate the relationship between laser intensity, preplasma and electron energy spectrum we will shortly show the results of modeling of the actual laser pulse incident on the plasma.

\subsection{Assumptions in the Electron Distribution}

These single exponential transport simulations show a method that gives an understanding of the electron acceleration changes with variations in the laser intensity and prepulse. The method has allowed quantitative assessments of electron spectra to be make for each point of data. However, hidden within these simulations are many assumptions about the electron distribution. First of all, we have assumed that the electrons will have the same spatial and temporal profile as the laser and that they have a $45^{\circ}$ divergence. Additionally, in this work, we assumed that the energy distribution of the electrons is a single exponential. However, this distribution could potentially be a Maxwell-Boltzmann distribution, a Maxwell-J'uttner distribution, the summation of multiple exponentials, or another type of function.

To get an idea of how these parameters change the inferred electron spectrum, we used the same interpolation method explained above. However, this time we tried a $10^{\circ}$ divergence and we tried adding a second exponential distribution that contained $50 \%$ of the energy. Again, we varied the temperature and the total electron energy and interpolated to get the best fit for our experiment.

Plotted in Figure 7.19 we show the best fits for the different assumptions. All of these different profiles appear to match the Trident data at $5 \times 10^{19} \mathrm{~W} / \mathrm{cm}^{2}$ quite well. However, we see that the slope temperature of the cooler electrons varied from 2.0 to $1.0 \mathrm{MeV}$, a difference of about $50 \%$. This shows how such 


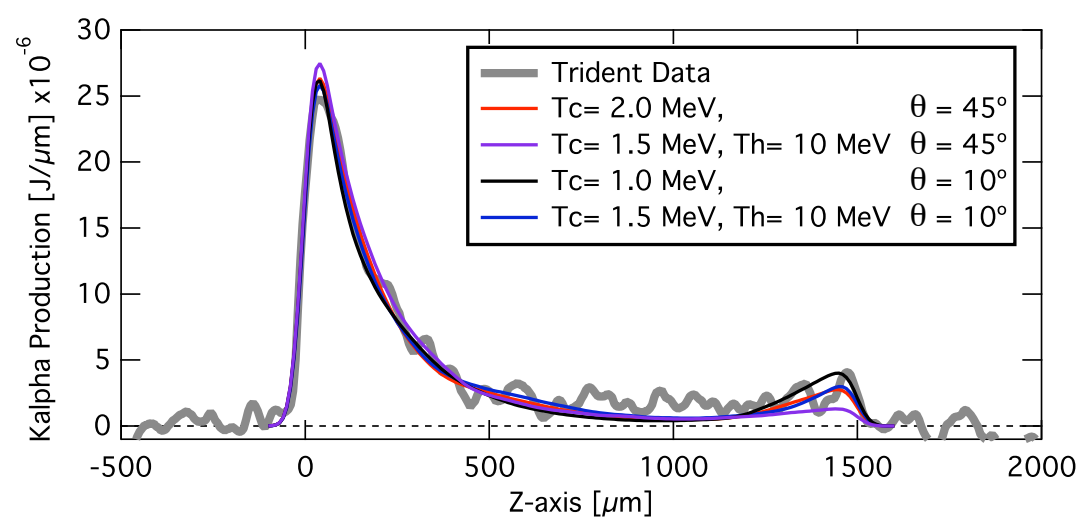

Figure 7.19: Fitting the $5 \times 10^{19} \mathrm{~W} / \mathrm{cm}^{2}$ shot Trident with a single exponential distribution while varying the angular distribution and injecting a hotter electron component with a $10 \mathrm{MeV}$ slope temperature.

assumptions affect the inferred slope temperatures.

The next step is to constrain these assumptions. This is done by modeling the electron spectrum consistently with the laser interaction. This will constrain the electrons in their temporal, spatial, angular and energy distributions, as based on the laser intensity and initial preplasma parameters. Such work will allow an understanding of the electron acceleration directly to determine the causes of the trends seen in the experimental data. 


\section{Laser-Plasma-Interaction Simulations}

This chapter shows the setup and results of laser-plasma-interaction (LPI) simulations to model the laser-acceleration of electrons. These simulations give an electron distribution that is well defined in time, space, angular divergence and energy; thus removing many of the assumptions that were made for the transport simulations presented in the previous chapter. Additionally, the role of preplasma in creating the electron distributions is investigated, both at high and low-contrast levels.

\subsection{LPI Modeling of Short-Scale Preplasma}

These laser-plasma-interaction (LPI) simulations again use the Particle-incell code LSP, ${ }^{[55]}$ as discussed in Chapter 4, now run including the fields of the laser. The setup of these simulations is shown in Figure 8.1 where we model the regions of the interaction where we expect the laser to play a large role. These simulations require a smaller cell size so that we can resolve the electro-magnetic fields of the laser, which also requires a much smaller time step. Thus we cannot model the full geometry of the target. We include the cone and preplasma extending $40 \mu \mathrm{m}$ from the cone tip, the $10 \mu \mathrm{m}$ cone-tip and the first $95 \mu \mathrm{m}$ of the wire. The cellsize is 20 times smaller than the laser wavelength (i.e. $50 \mathrm{~nm}$ ) and the time step is the laser period divided by 133 . We included species of $\mathrm{Au} / \mathrm{Cu}$ ions and electrons, which were initialized with 10 and 50 particles per cell, respectively. The Au was initialized at a charge state of 10 and was allowed to ionize via the ADK ${ }^{[59]}$ model 
using tabulated ionization energies, ${ }^{[58]}$ as described in Section 2.2.1.

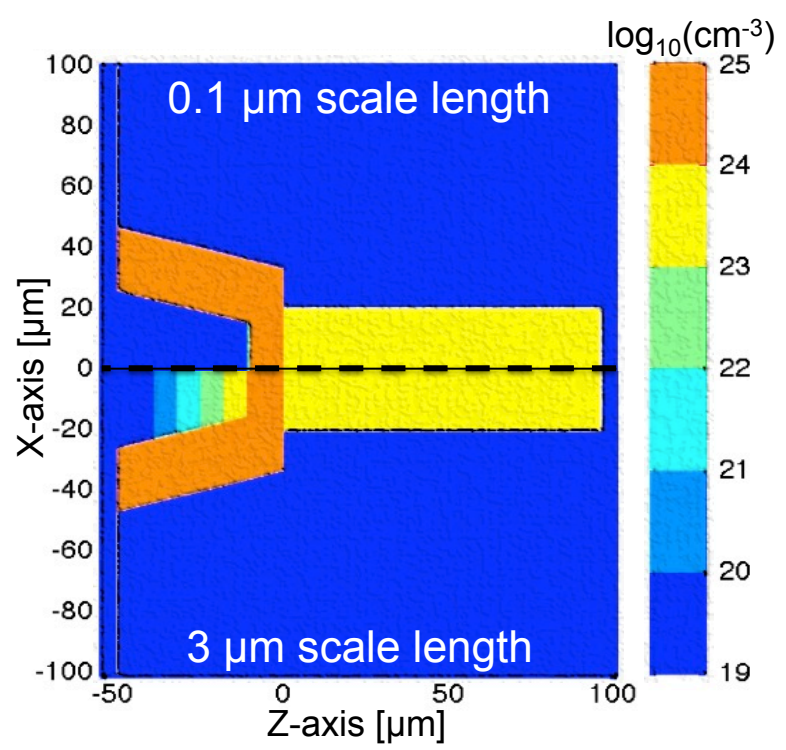

Figure 8.1: Electron number density in the initial setup of the LPI simuations with preplasma scale lengths of 0.1 and $3.0 \mu \mathrm{m}$.

The spatial profile of the laser is given by the summation of three gaussian functions as we have described in Table 3.4 of Section 3.1.2, and the temporal function is a single gaussian with the same profile as the experiment. Unlike the transport simulations, a 2D Cartesian geometry is used to model the LPI, because the laser cannot propagate in a 2D cylindrical geometry. For this reason, modeling of the transport will not be done in these simulations and, instead, the electron distribution will be handed off to transport simulations to perform comparisons to experimental data.

One of the main uncertainties in the experiments is the amount of preplasma that is ablated prior to the arrival of the main pulse of the laser. At high-contrast, this is extremely difficult to measure, as the scale length is very short. It is also difficult to model, since it is very dependent on the absorption and EOS of materials, which are not always well known. In the high-contrast case of Trident, most of the preplasma is caused by the rising-time of the laser, which rises less than 0.1 ns before the main pulse. Since this rising-time is quite fast and the preplasma is fairly short, it is difficult to estimate its scale length. In order to bracket this 
uncertainty, we have run three different cases at the highest $5 \times 10^{19} \mathrm{~W} / \mathrm{cm}^{2}$ case on Trident for $0.1,1$ and $3 \mu \mathrm{m}$ of exponential preplasma. The 0.1 and $3 \mu \mathrm{m}$ cases are shown in Figure 8.1. Additionally, as discussed further in the text, we will model the low-contrast Titan laser with a much longer scale length of preplasma.

\subsection{Absorption of Laser Energy}

One way to understand the effect of changing the preplasma scale length is to look at the amount of laser energy absorbed in the simulations and the energy coupled to relativistic electrons that reach the cone-tip. The laser absorption is determined by subtracting the laser energy leaving the simulation from the laser energy input into the simulation. The electron coupling is determined by integrating the net (forwards minus backwards going) electron energy from a plane at the cone-tip.

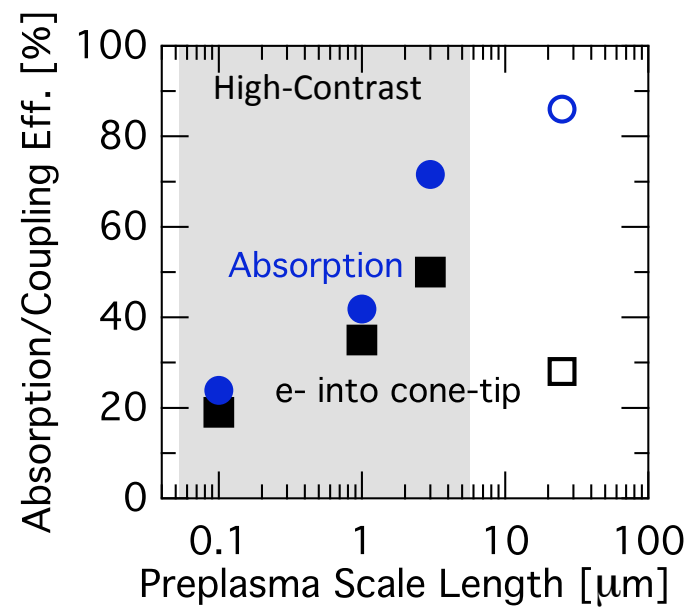

Figure 8.2: Absorption (circles) and coupling (squares) efficiency of electrons into the cone-tip. The closed markers represent the high-contrast data by using 0.1 to $3 \mu \mathrm{m}$ exponential profiles and the open markers represent the low-contrast case with much longer preplasma.

We show the variation of the absorption and the coupling efficiency in Figure 8.2 as function of the preplasma scale length. These simulations show that increasing the preplasma levels from 0.1 to $3 \mu \mathrm{m}$ increases the absorption from 
$20 \%$ to $70 \%$. On the other hand, in the same cases, the electron energy passing through the tip (solid squares) only increases from $20 \%$ to $50 \%$. This shows that electrons generated in simulations with higher levels of preplasma have more difficulty reaching the cone-tip. At lower levels of preplasma, up to $3 \mu \mathrm{m}$, this effect is offset by the increased total absorption of electrons. However, with larger, Titanlike, levels of preplasma (open circles/squares) this increased difficulty in reaching the cone-tip leads to a lower amount of energy passing through the tip. This is consistent with the experimental trends previously presented in Figure 5.5, which indicate that low-contrast lasers have a 3 times lower coupling into a wire attached to the cone.
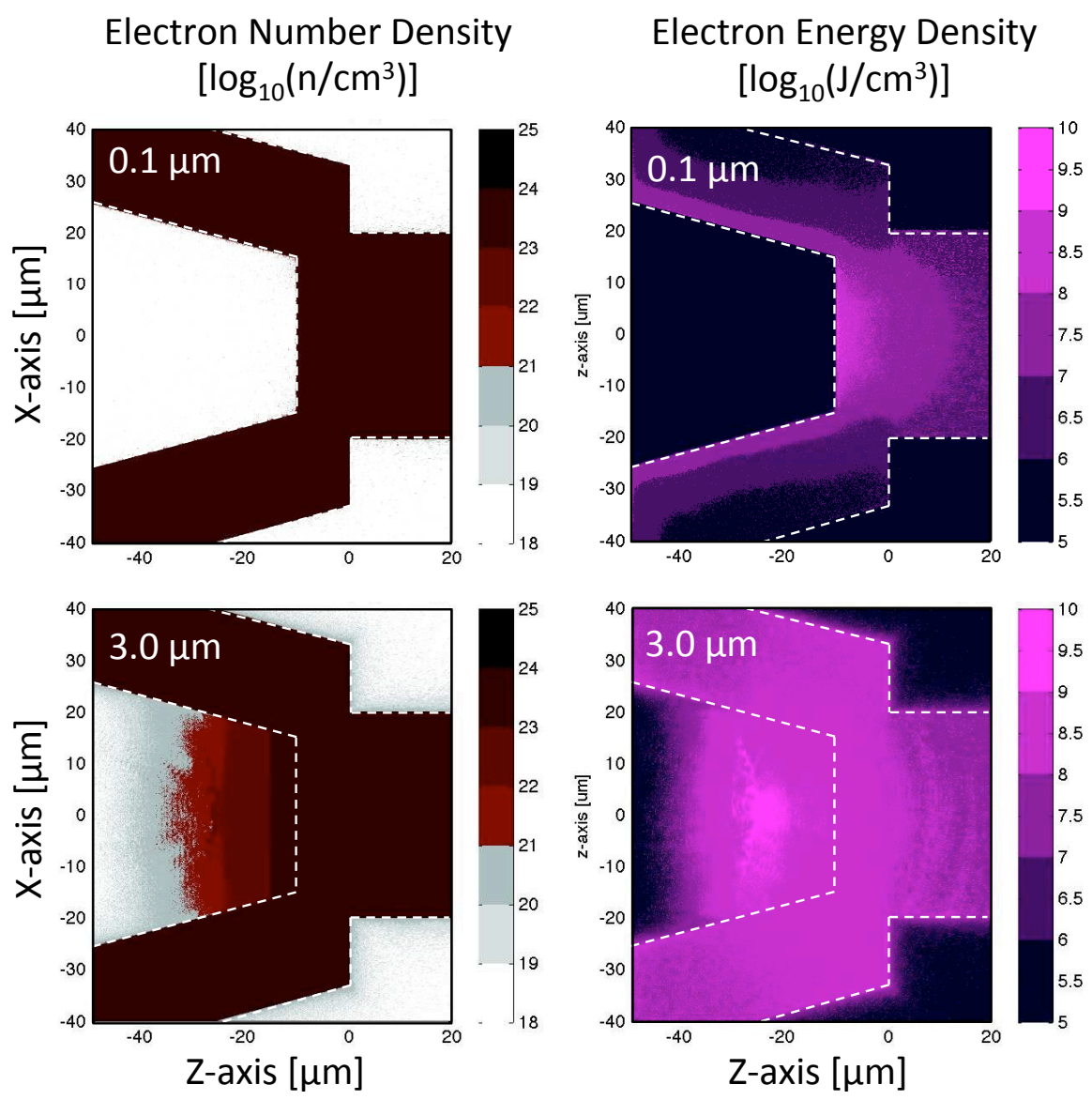

Figure 8.3: Number density and energy density of electrons in the LPI simulations of 0.1 and $3 \mu \mathrm{m}$ preplasma near the peak of the pulse at $1 \mathrm{ps}$. The dotted white represents the outline of the inner cone wall. 
The electron number density and energy density for the 0.1 and $3 \mu \mathrm{m}$ cases are shown in Figure 8.3 near the peak of the pulse at 1 ps. The color scale used in the number density plots show densities above critical density in red and lower densities in gray. The critical density in the $0.1 \mu \mathrm{m}$ case is very close to the initial cone-tip, while in the $3 \mu \mathrm{m}$ case it is located about $20 \mu \mathrm{m}$ from the tip. Additionally, the $3 \mu \mathrm{m}$ case has a much larger amount of under-dense plasma in which the electrons can be accelerated. This is seen in the wider spread of the electron energy distribution in space.

As was discussed previously, the electron energy distribution from the $3 \mu \mathrm{m}$ case shows a much large total amount of electron energy, but these electrons are divergence and are spread across a large portion of the cone. In contrast to this, the $0.1 \mu \mathrm{m}$ preplasma case shows less energy in electrons, but the majority of the electron energy is much more localized near to the cone-tip. Interestingly, this trade-off between the total amount of electron energy absorbed and the distance that the electrons are accelerated away from the cone-tip suggests that there is an optimal preplasma scale length. This scale length would be found by optimizing the laser absorption, while keeping the location of electron-accelerated as close as possible to the cone-tip.

\subsection{Accelerated Electron Distributions}

In the previous chapter, we inferred the electron spectra by injecting multiple distributions until they matched the experimentally measured falloffs of $\mathrm{K}_{\alpha}$ emission along the $\mathrm{Cu}$ wire. This method required assumptions to be made for the temporal, spatial and angular distributions of the electrons. As shown in Section 7.7, making different assumptions changed the electron spectrum that was inferred. In this section, we present the temporal, spatial, angular and energy distributions of the accelerated electrons as derived from the LPI simulations. The electrons are observed by collecting the electrons that pass through the tip of the cone. 


\subsubsection{Spatial and Temporal Profiles}

Figure 8.4 shows spatial and temporal distributions of electrons from the 0.1 and $3 \mu \mathrm{m}$ preplasma cases, along with the corresponding incident profiles of the laser. With a $0.1 \mu \mathrm{m}$ preplasma the electron profiles are similar to the incident laser profile. For instance, the electron energy distribution in space has a full-
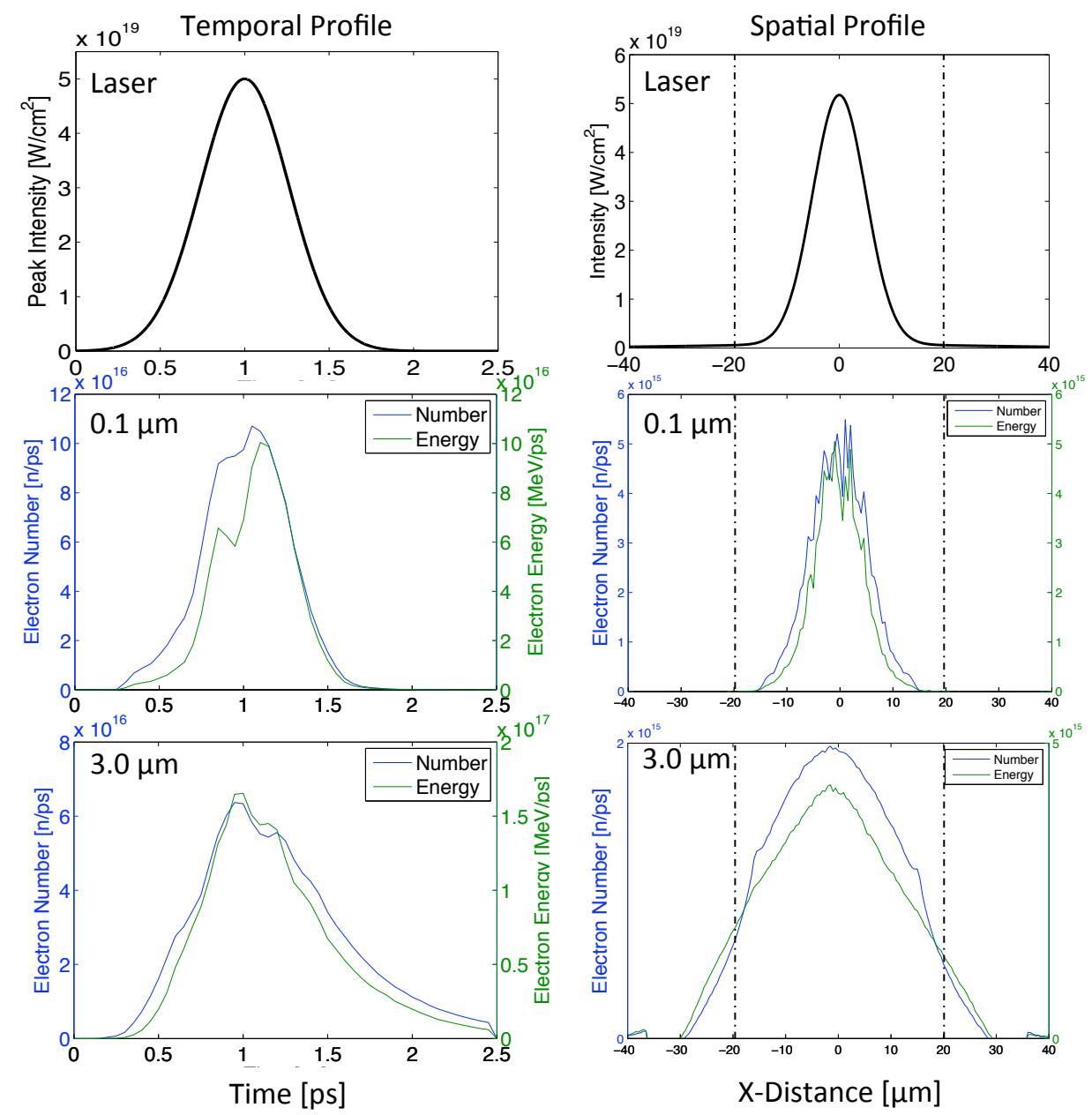

Figure 8.4: Time and spatial profiles of electrons going through the cone tip for the 0.1 and $3.0 \mu \mathrm{m}$ cases. Plots on the bottom show the laser distribution. The dotted lines in the spatial plots show the width of the wire.

width-at-half-maximum (FWHM) of $12 \mu \mathrm{m}$ which is nearly identical to the laser system, as described in Chapter 3. On the other hand, the electron profiles for the $3 \mu \mathrm{m}$ case are significantly broadened compared to the laser profile. In the $3 \mu \mathrm{m}$ 
case, the energy distribution has a FWHM of $33 \mu \mathrm{m}$ in space, which is about three times wider than the $0.1 \mu \mathrm{m}$ case. This broadening is due to the electrons being initially accelerated at distances further away from the tip of the cone, as shown in Figure 8.3. Since the electrons have a given angular divergence, this distance gives the electrons more distance over which to separate. Additionally, this divergence may change with preplasma, as will be discussed shortly.

The spatial profiles in Figure 8.4 include a dotted line at $\pm 20 \mu \mathrm{m}$ from the center to represent the dimensions of the $\mathrm{Cu}$ wire attached to the outer cone-tip. This is important, as only electrons that pass through the $\mathrm{Cu}$ wire will generate $\mathrm{K}_{\alpha}$ x-ray emission and, thus be measured by the experimental diagnostics. Even though some electrons are outside of this regions in the $3 \mu \mathrm{m}$ case, most of the energy is contained within this region, which means that our diagnostics are sensitive to a large portion of the electrons generated by the laser. This is an advantage of using high-contrast lasers; since the accelerated electrons start closer to the cone-tip, they should pass through the wire and thus be well constrained by the experimental measurements.

\subsubsection{Angular Distribution}

The increased width of the spatial distribution in the $3 \mu \mathrm{m}$ case may be amplified by an increased initial divergence of the electrons. Figure 8.5 shows the average divergence of the electrons in the different cases. The divergence angle $\theta$ is calculated as $\theta=\cos ^{-1}\left(p_{z} / p\right)$, where $p_{z}$ is the electron's momentum in the forward direction and $p$ its total momentum. At lower energies, both cases show average electron divergence angles of around $50^{\circ}$, and both cases show that the divergence angle of electrons decreases as the electrons increase in energy. This behavior is consistent with the ejection angle derived in Section 2.3.3 for the $\mathbf{j} \times \mathbf{B}$ acceleration mechanism, which also shows that higher energy electrons will have less divergent ejection angles.

Figure 8.5 also shows that the average angles of the electrons are larger with more preplasma. One potential reason for this is the presence of large quasi-static magnetic fields as shown in Figure 8.6. In this figure, the magnetic fields have 


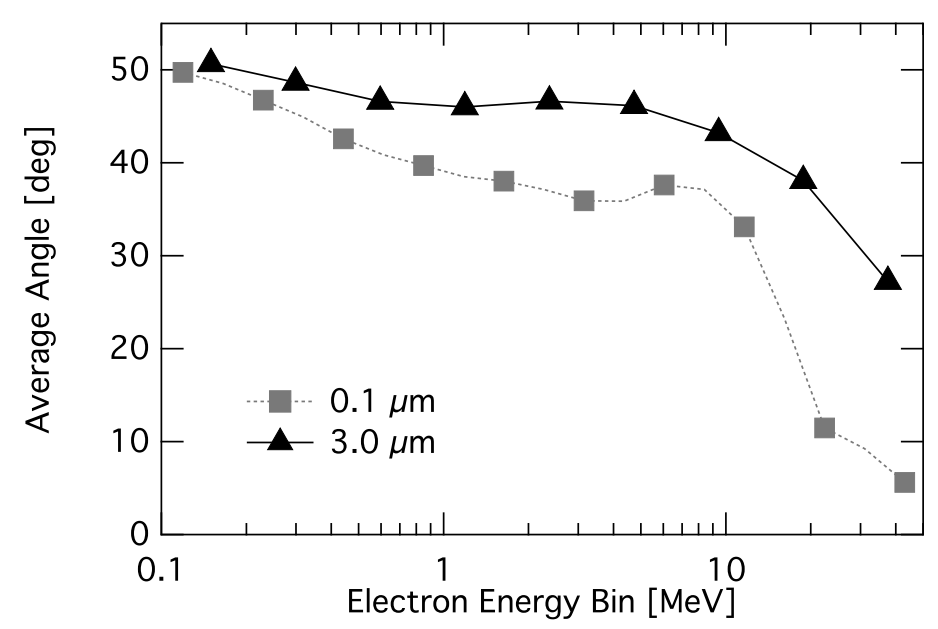

Figure 8.5: Average angles of the electrons in the 0.1 and $3.0 \mu \mathrm{m}$ preplasma cases.

been averaged along the $z$-axis to smooth over the periodic fields due to the laser propagation. The magnetic fields are stronger and exist over longer distances in

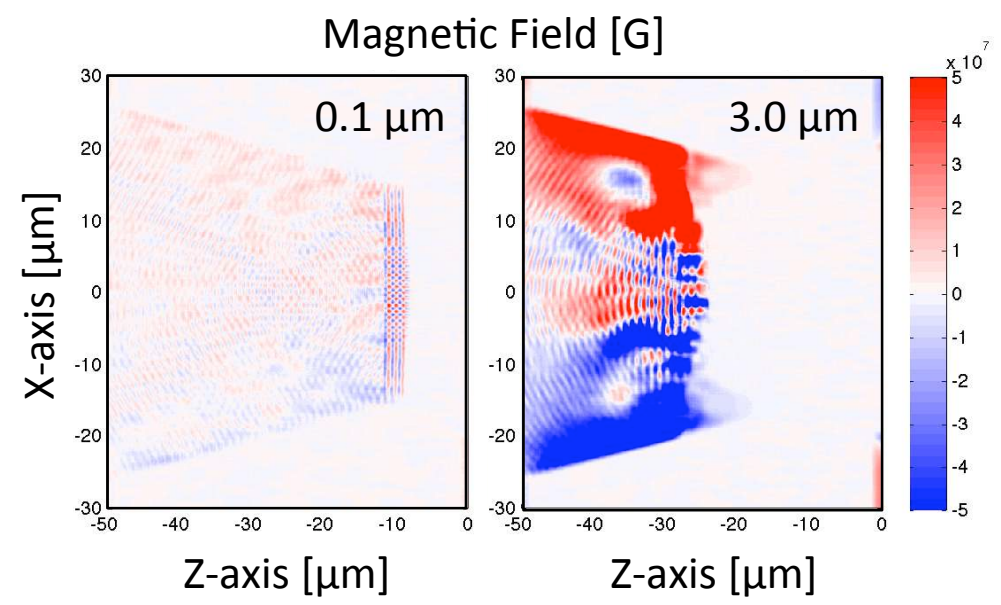

Figure 8.6: Quasi-static, smoothed magnetic fields near the peak of the laser pulse at 1 ps.

the $3 \mu \mathrm{m}$ case than in the $1 \mu \mathrm{m}$ case. These magnetic fields have strengths as high as $50 \mathrm{MG}$, which is strong enough to substantially turn electrons. For reference, the Larmor radii for electrons with kinetic energies of 1 and $10 \mathrm{MeV}$ are 1 and $7 \mu \mathrm{m}$, respectively, in a $50 \mathrm{MG}$ field. Thus this mechanism could be responsible for the increased divergence with magnetic fields. An additional mechanism to 
increase divergence are the ripples in the critical density that can be observed in the electron density plots in Figure 8.3. These ripples can modify the phase fronts of the incident laser and thus inject the electrons at more divergent angles.

\subsubsection{Energy Spectrum}

A feature of the accelerated electrons that is well constrained by the conewire targets, and that is of particular interest to fast ignition research, is the electron energy spectrum. As shown previously in Chapters 7 and 6 , the falloff of $\mathrm{K}_{\alpha} \mathrm{x}$-ray emission in the wire is able to constrain the energy spectrum. Understanding this spectrum is of great interest to the fast ignition so that the energy deposition within the imploded core can be optimized.

The electron spectrum is obtained using a similar method to the temporal and spatial distributions, where forward traveling electrons are counted as they cross the cone-tip and backwards traveling electrons are subtracted. In addition to this, an adjustment is made for the radial weight of the electrons. Recall that the laser was injected into the LPI simulations to match the spatial profile measured in the experiment. Thus, since we are using a 2D Cartesian geometry, this cannot also match the energy distribution of the experiment. To correct for this, we apply a radial weighting to the electron distribution by multiplying by $r$ the distance from the central axis.

The electron spectra for the 0.1 and $3 \mu \mathrm{m}$ cases are shown in Figure 8.7. These spectra are fit with a three-temperature exponential to quantify the changes that occur to the electron spectra with increasing prepulse. The fits to the spectra are shown in Table 8.1. The need to use multiple exponential distributions to characterize the electron spectrum shows that the single exponential distributions assumed in the transport simulations of Chapter 7 do not accurately represent the spectra, despite the fact that such a distribution is consistent with the experimental data as shown in Figure 7.17.

With this laser $\left(I \lambda^{2}=5.6 \times 10^{19} \mathrm{Wcm}^{-2} \mu^{2}\right)$ the ponderomotive energy (from $\mathbf{j} \times \mathbf{B}$ force, derived in Section 2.3.3) is $2.78 \mathrm{MeV}$. This is a similar to the temperature of the hottest portions of the spectrum $\left(T_{3}\right)$ for both the 0.1 and 3 

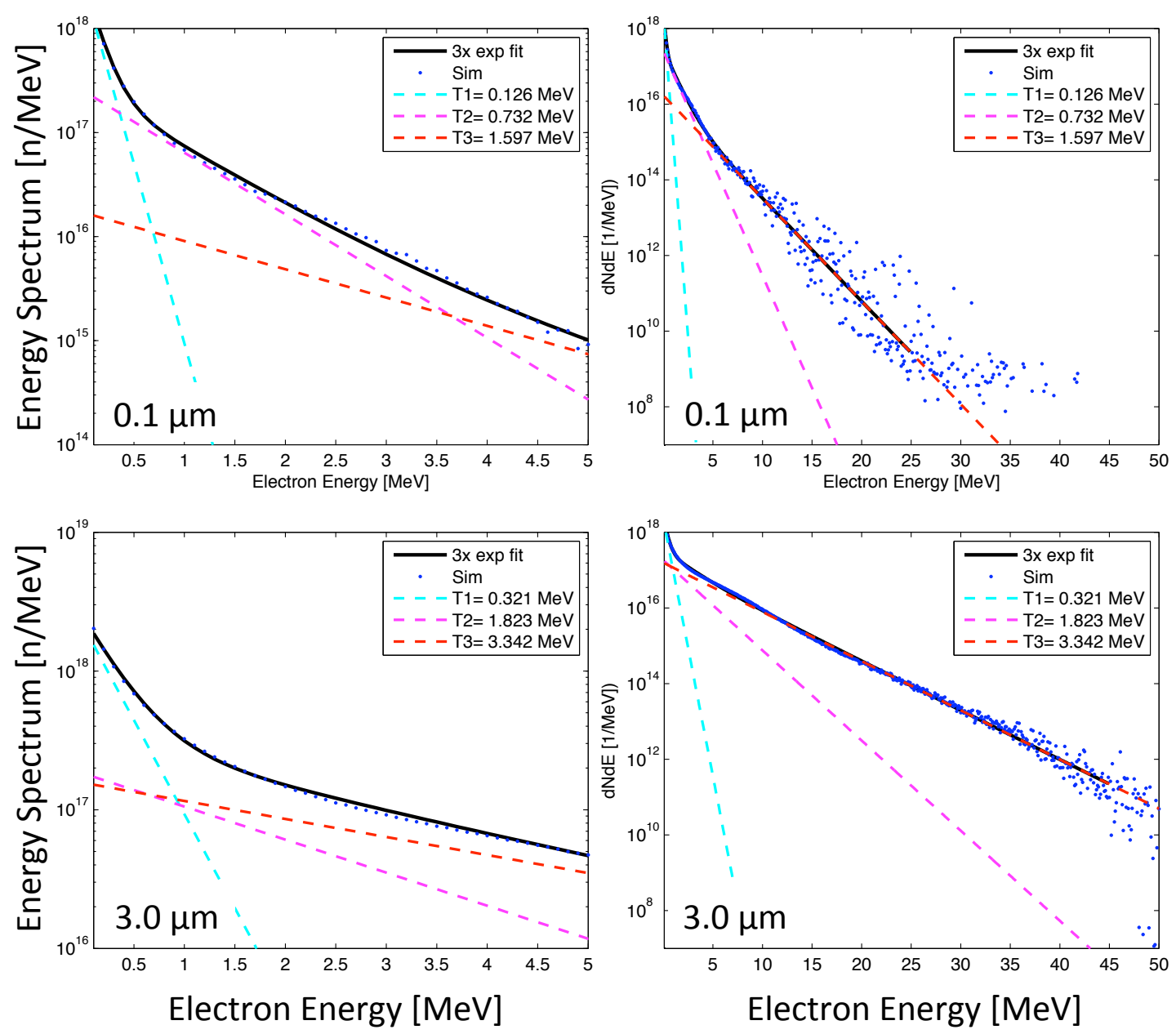

Figure 8.7: Energy spectrum of the electrons passing through the cone-tip for the 0.1 and $3 \mu \mathrm{m}$ cases, shown for two different energy windows.

$\mu \mathrm{m}$ cases, which suggest that these are accelerated by the $\mathbf{j} \times \mathbf{B}$ force. On the other hand, the lower portions of the spectrum $\left(T_{1}\right)$ have slope temperatures on the order of a few hundred keV, which are cooler than the ponderomotive energy, and thus may not be generated through the $\mathbf{j} \times \mathbf{B}$ mechanism.

The LPI simulations show that increasing the preplasma scale length increases the slope temperature of the electrons, as well as shifting the fraction of the total energy towards higher energies. This shows that preplasma, as well as laser intensity, plays a major role in electron acceleration. For a 30x increase in preplasma scale length the slope temperatures increase by a factor of 2 to $2.5 \mathrm{x}$. 
Table 8.1: Parameters of triple exponential fits to the electron spectra. Temperatures $T$ and fraction of energy $F$ contained for each exponential.

\begin{tabular}{cc|cccccc}
$\begin{array}{c}I_{0} \\
{\left[\mathrm{~W} / \mathrm{cm}^{2}\right]}\end{array}$ & $\begin{array}{c}\text { Scale } \\
{[\mu \mathrm{m}]}\end{array}$ & $\begin{array}{c}T_{1} \\
{[\mathrm{MeV}]}\end{array}$ & $F_{1}$ & $\begin{array}{c}T_{2} \\
{[\mathrm{MeV}]}\end{array}$ & $F_{2}$ & $\begin{array}{c}T_{3} \\
{[\mathrm{MeV}]}\end{array}$ & $F_{3}$ \\
\hline $5 \cdot 10^{19}$ & 0.1 & 0.126 & $18 \%$ & 0.732 & $62 \%$ & 1.597 & $20 \%$ \\
& 1.0 & 0.216 & $10 \%$ & 0.864 & $45 \%$ & 3.468 & $45 \%$ \\
& 3.0 & 0.321 & $8 \%$ & 1.823 & $24 \%$ & 3.342 & $68 \%$
\end{tabular}

Recall that the coupling of electrons in the cone-tip also increases by a factor of 2.5x as shown in Figure 8.2. The total number of electrons that pass through the cone-tip is $6.7 \times 10^{16}$ and $6.1 \times 10^{16}$ for the 0.1 and $3 \mu \mathrm{m}$ cases, respectively. Thus an increased preplasma level does not cause a larger number of electrons to be accelerated, but does cause individual electrons to gain more energy. This is presumably due to a longer distance over which the laser can interact with the electron since electrons as there are many more electrons at locations where the density is below critical.

The LPI simulations have shown how changing the scale length of preplasma changes the spatial, temporal, angular and energy distribution of accelerated electrons. These show that increased preplasma increases the absorbed fraction of laser light by increasing the energy that electrons gain from the laser. However, the larger distance from the cone-tip at which the electrons are born, as well as, their increased divergence, means that the fraction of the accelerated electrons that reach the tip decreases with preplasma. The simulations have shown that all of the characteristics of the electrons are coupled; and all of them depend on the scale length of preplasma in the simulation. In the simulations of the electron transport in Chapter 7 we assumed that these were kept constant and that they could be described by simple analytical functions. Now we move forward from these simple models and use the LPI generated distributions to compare with the experimental data. 


\subsection{Transport Simulations of LPI-measured Elec- tron Distributions}

Simulations of the laser-plasma-interaction have now been performed to understand the spatial, temporal, angular and energy distributions of the accelerated electrons. These will now be compared to experimental data by injecting these electrons into transport simulations. As was discussed in Chapter 7, it is important to use a 2D cylindrical geometry and to model the full scale of the target, thus the electron distributions from the LPI simulations must be transfered to the electron transport simulations. This is done by creating a number of logarithmically spaced kinetic-energy bins, and then determining the spatial, temporal, angular and energy distribution for each bin. These electron distributions are then injected at the cone-tip of the transport simulations, which include all of the physics discussed previously in Chapter 7. These simulations are run with varied electrons energies so that the total amount of $\mathrm{K}_{\alpha}$ emission matches the experimentally measured data.

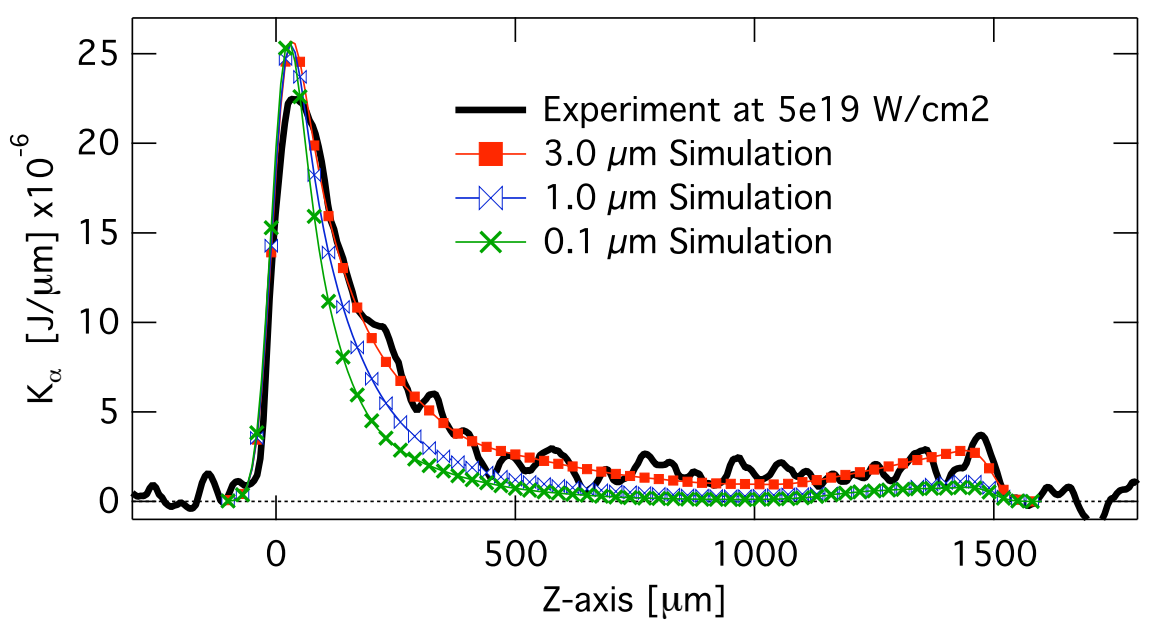

Figure 8.8: Profile of $\mathrm{K}_{\alpha}$ emission from the wire using the injected electron sources from the 0.1, 1 and $3 \mu \mathrm{m}$ preplasma LPI simulations and the experimental data for the $5 \times 10^{19} \mathrm{~W} / \mathrm{cm}^{2}$ case.

The $\mathrm{K}_{\alpha}$ emission profiles are shown in Figure 8.8. This shows that the elec- 
tron spectrum generated by $3 \mu \mathrm{m}$ preplasma simulation is the best match to the experimental data. This is somewhat unexpected, as previous work ${ }^{[18]}$ assumed that preplasma scale length was on the order of $0.5 \mu \mathrm{m}$ for ultra-high-contrast lasers. However, our simulations show that a scale length of 0.1 or even $1 \mu \mathrm{m}$ does not create an electron spectrum hot enough to match falloff observed experimentally.

To compare the LPI inferred energy spectrum with the single exponential injections from of Chapter 7 , the best fits to the experimental data are plotted in Figure 8.9. The striking similarity of the spectra between 1 and $4 \mathrm{MeV}$ shows the ability of the wires to constrain the electron spectrum at these energies. This is due to the strong dependence of the falloff on the electron spectrum at these energies as discussed in Section 6.1 and highlights the robustness of the cone-wires as a diagnostic for such energies.

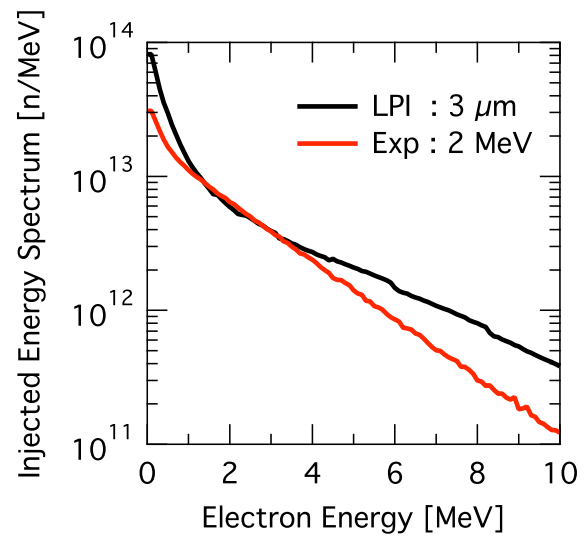

Figure 8.9: Comparison of the electron spectrum inferred from LPI modeling and the spectrum inferred from single temperature injections for the $5 \times 10^{19} \mathrm{~W} / \mathrm{cm}^{2}$ Trident case.

To understand how the electrons scale with intensity, preplasma scale lengths of $0.1,1.8$ and $3 \mu \mathrm{m}$ were performed at $2 \times 10^{19} \mathrm{~W} / \mathrm{cm}^{2}$ intensity. This is the lowest intensity in our high-contrast experiments. The same procedure is used to inject the electron spectrum into the transport simulations. Figure 8.10 shows the corresponding $\mathrm{K}_{\alpha}$ profiles. At this intensity, a $3 \mu \mathrm{m}$ preplasma yields electrons too hot to fit the experimental data. Instead, the optimal fit is near $1.8 \mu \mathrm{m}$ scale length. 


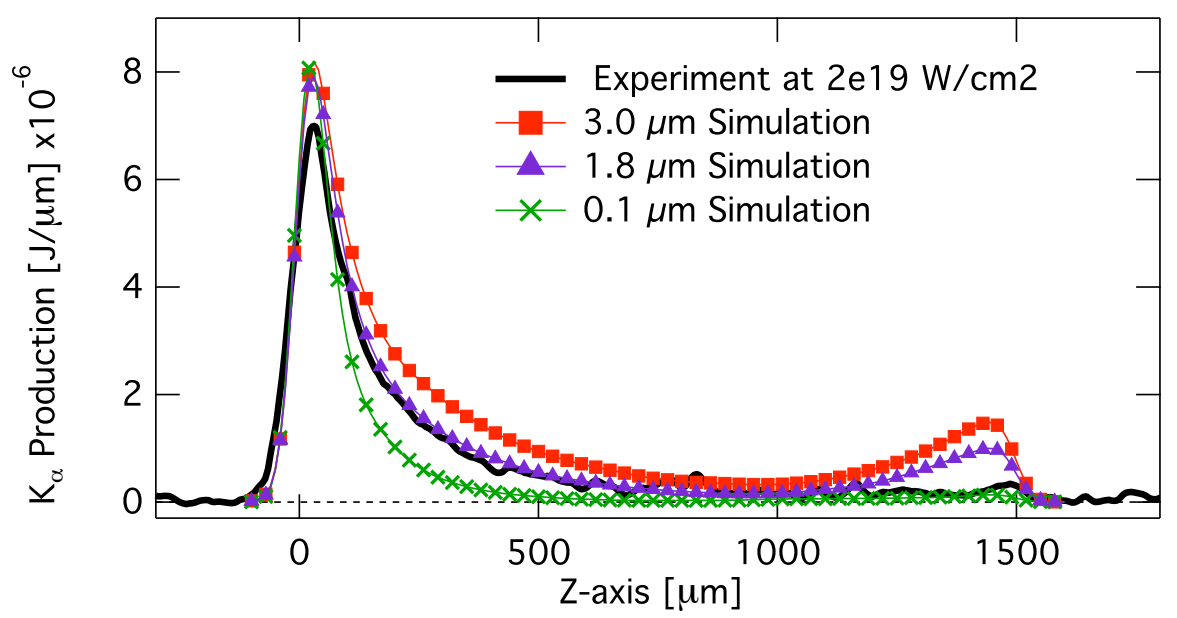

Figure 8.10: Profile of $K_{\alpha}$ emission from the wire using the injected electron sources from the $0.1,1.8$ and $3 \mu \mathrm{m}$ preplasma LPI simulations and the experimental data for the $2 \times 10^{19} \mathrm{~W} / \mathrm{cm}^{2}$ case.

This implies that a different amount of preplasma is present at different intensities. In the experiments, the laser intensity is varied to change the intensity, so it is realistic to assume that this changes the energy in the $0.1 \mathrm{~ns}$ rising-time of the laser and affects the preplasma generation. This shows varying the intensity alone is not enough to reproduce the electron energy trends observed experimentally; instead, the preplasma must also be modified.

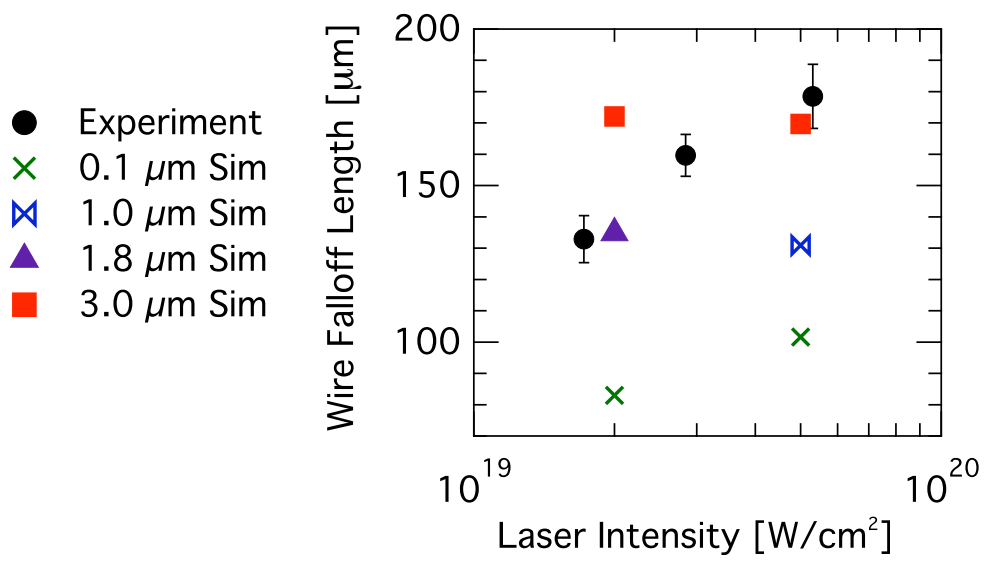

Figure 8.11: Falloff distance of $\mathrm{K}_{\alpha}$ emission from the simulations at varying preplasma scale lengths plotted with the experimental data. 
To quantify these comparisons, the simulated $\mathrm{K}_{\alpha}$ profiles are fit with an exponential profile and the corresponding falloff distances are plotted with the experimental data in Figure 8.11. This data implies that there is a 1.8 to $3 \mu \mathrm{m}$ preplasma present on Trident. As this is significantly larger than the preplasma expected from previous work, ${ }^{[18]}$ we now go through a simple analytical model to estimate whether such a large scale length of preplasma is physically reasonable on Trident.

\subsection{Preplasma Expansion Model}

As the preplasma scale length inferred from these high-contrast experiments is somewhat higher than previously expected, ${ }^{[18]}$ an order of magnitude estimate is now shown to provide confidence that the inferred scale length is physically reasonable. Unfortunately, such low laser intensities during short ( $>0.1 \mathrm{~ns}$ ) time scales are difficult to simulate due to uncertainties in the absorption and equationof-state of the material. Thus, an analytical model will be used to provide an order-of-magnitude estimate to show if the 1.8 to $3 \mu \mathrm{m}$ scale are reasonable.

Let us assume that the preplasma is expanding thermally ${ }^{[60,61]}$ with a selfsimilar solution ${ }^{[62,92]}$ with the profile, $n_{i} \propto \exp \left(\frac{x}{\lambda_{p}}\right)$ where $\lambda_{p}=C_{s} t, C_{s}=$ $\sqrt{Z T / m_{i}}$ is the sound speed of the gas, $Z$ is the average charge state, $T$ is the temperature and $m_{i}$ is the ion mass. To generalize this, we assume a temperature that varies in time and thus the scale length is $\lambda_{p}(t)=\int_{t_{0}}^{t} C_{s}(t) d t^{\prime}$. We add an amount of energy per unit mass for a given time $d Q$ using,

$$
\frac{d Q}{d t}(t)=\frac{\eta(I) \times I(t)}{M(t)}
$$

Here $I$ is the laser intensity, $\eta$ is the absorption fraction of laser energy, and $M$ is the mass per unit area, which we call the mass for brevity. The intensity is taken from the measured contrast profile, ${ }^{[18]}$ shown in Section 3.1.3, and the absorption is calculated as a function of intensity. ${ }^{[92]}$ The calculation of mass is a very important. Essentially, this depends on the depth at which the energy is absorbed and the density at which this absorption happens. It is important, because, as the mass becomes close to zero the heat per unit mass added to the 
system will increase to infinity and the system will explode. To avoid this, we use a two-limit model that prevents the mass from going to zero by identifying two extremes that are likely to occur. The first extreme happens when the laser is incident on an sharp interface, at this point the energy of the laser is absorbed into a skin depth $\delta$, which is defined as $\delta=c / \omega_{p e}$. Where $\omega_{p e}^{2}=\left(4 \pi q_{e}^{2} / m_{e}\right) Z n_{i 0}$, and $q_{e}$ is the electron charge, $m_{e}$ is the electron mass, $n_{i 0}$ is the ion density of solid gold, and $c$ is the speed of light in vacuum. For reference, the skin depth of solid gold is $22 \mathrm{~nm}$ at $Z=1$ and $2.5 \mathrm{~nm}$ at $Z=79$.

As the gold expands into vacuum, we build up an exponential falloff, as described above, with a gradient $\lambda_{p}$. For our second limiting case, we assume that laser is absorbed in this exponential falloff that extends up to the critical density $n_{c}$ of the plasma. Thus we integrate the mass from infinity until the critical density $M=m_{i} \int_{-\infty}^{x\left(n_{c}\right)} \exp \left(\frac{x}{\lambda_{p}}\right) d x^{\prime}=m_{i} n_{c} \lambda_{p}$ to get the amount of mass that the laser will be absorbed into. To combine these two extremes we take the maximum of either of them:

$$
M=m_{i} \times \max \left(n_{i 0} \delta, n_{c} \lambda_{p}\right)
$$

The final step is to get the temperature and ionization state from the amount of energy added to the system. We use the equation-of-state ${ }^{[85,86]}$ that was described previously in Section 4.4 to get the temperature and ionization state of the material. In this model we do not include the energy lost through radiation and conduction of energy is neglected. These models should be viewed as a order of magnitude estimate to determine whether are inferred scale lengths are physically reasonable.

The preplasma scale lengths $\lambda_{p}$ calculated from this model are 3.2 and 3.8 $\mu \mathrm{m}$ for the $2 \times 10^{19}$ and $5 \times 10^{19} \mathrm{~W} / \mathrm{cm}^{2}$ cases. This is comparable to 1.8 and $3 \mu \mathrm{m}$ for the same cases inferred from the LPI simulations. Thus our calculation gives confidence that the preplasma cases inferred though our modeling are physically reasonable. 


\subsection{LPI Modeling of Large-Scale Preplasma}

In addition to the modeling of the high-contrast Trident cases, we are also interested in understanding the results from the experiment performed on the lowcontrast Titan laser. Recall that the experimental data in Chapter 5 showed two major differences between the high and low-contrast data. First the coupling of energy into $\mathrm{K}_{\alpha} \mathrm{x}$-ray emission was $2.7 \mathrm{x}$ higher using high-contrast, indicating a similar increase of electron energy coupling into the wire. Second, the falloff of $\mathrm{K}_{\alpha} \mathrm{x}$-ray emission was much shorter at low-contrast indicating that electron slope temperature within the wire is cooler. Using single exponential spectra in the transport simulations of Chapter 7 , such trends were shown be a function of the electron spectrum and not simply artifacts of the diagnostic. To investigate further, we look into the acceleration of electrons in the low-contrast case to identify the causes of these effects.

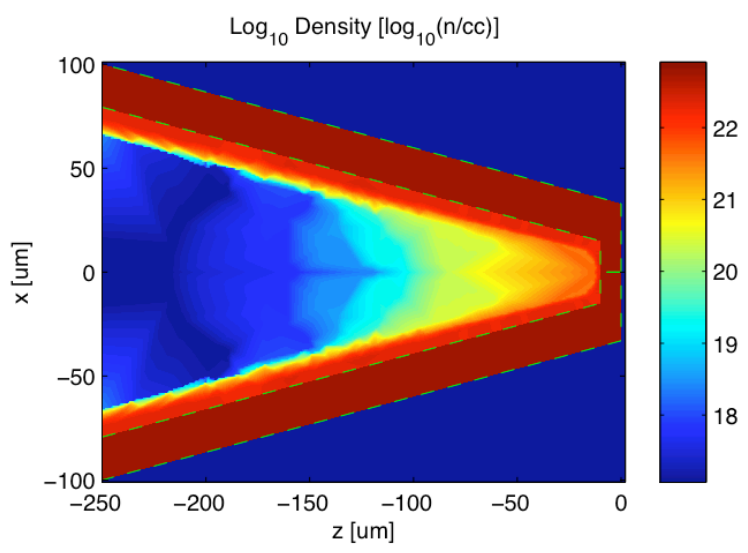

Figure 8.12: Preplasma density map expected on the Titan laser with $17 \mathrm{~mJ}$ of prepulse energy in 2 ns.

The low-contrast Titan case has a much larger amount of preplasma than the previous simulations, and as the prepulse is $2 \mathrm{~ns}$ in duration, such time scales are feasible to model hydrodynamically. To obtain a preplasma profile consistent with a Titan prepulse, hydrodynamic modeling was performed by S. R. Chawla running the code HYDRA. The laser injected into this simulation has $17 \mathrm{~mJ}$ of energy in a 2 ns pulse, which is representative of the Titan prepulse. This code 
uses an equation-of-state to model the ionization of the material and allows the preplasma to expand and absorb laser light. The electron density map created in this simulation is shown in Figure 8.12. Notice that the critical density of $10^{21}$ $\mathrm{cm}^{-3}$ is already $50 \mathrm{\mu m}$ away from the cone-tip, which is too far back to fit in the previously used simulation-box. Thus the size of the simulation is increased to include longer preplasma. However, other than this difference, the simulations to model the Titan pulse are the same as run for high-contrast.

\subsubsection{Coupling at Low-Contrast}

At the beginning of this chapter, Figure 8.2 showed that a large amount of preplasma increases the laser absorption, but decreases the fraction of the absorbed energy into electrons that reach the cone-tip. This was shown to reduce the energy of electrons reaching the wire from a peak of 50\% using a $3 \mu \mathrm{m}$ preplasma, to $30 \%$ in the Titan-like low-contrast case. This $30 \%$ coupling means much electron energy is lost or does not reach the cone, compared to the $90 \%$ absorption of laser light.

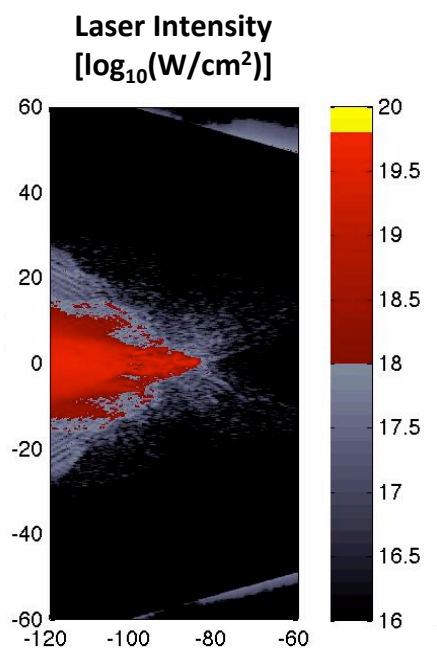

Elec. Num. Den. $\left[\log _{10}\left(\mathrm{~cm}^{-3}\right)\right]$

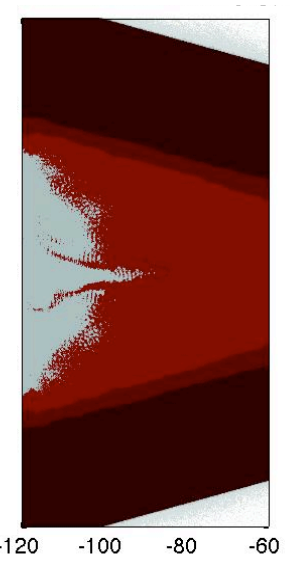

Elec. Energy Den. $\left[\log _{10}\left(\mathrm{~J} / \mathrm{cm}^{3}\right)\right]$

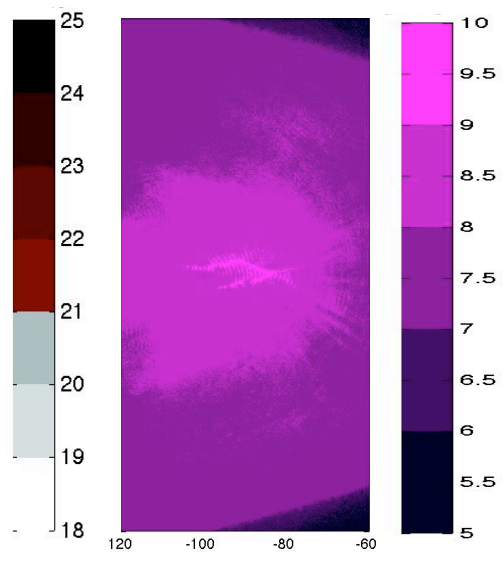

Figure 8.13: Profiles of the laser intensity, electron number density and electron energy density of the LPI simulations representing low-contrast Titan interaction near the peak of the pulse.

To understand the mechanisms of this decrease, the Figure 8.13 shows the 
laser intensity, electron number density and electron energy density of the longscale Titan-like preplasma LPI simulation at a time near the peak of the pulse. In these simulations the critical density is about $70 \mu \mathrm{m}$ from the cone-tip in contrast to only $20 \mu \mathrm{m}$ in the $3 \mu \mathrm{m}$ preplasma case shown in Figure 8.3. This is one cause for the decreased amount of electron energy reaching the cone-tip. This figure also shows a strong modification of the plasma by the laser itself. For instance, the electron number density map shows a large channel formed in the center of the plasma, this is formed by the hole-boring mechanism discussed in Section 2.3.2, where over multiple cycles, the laser pushed electrons out of regions of higher intensity. Additionally, the laser intensity map shows some filamentation of the beam as it propagates through the plasma. Both the filamentation and hole-boring processes are imprinted on the accelerated electrons as can be seen in the electron energy density map, which shows electrons being generated from within the channel and shows a number of small filamentary structures.

These simulations look substantially different than high-contrast simulations of 0.1 to $3 \mu \mathrm{m}$ preplasma shown previously. These previous simulations showed little modification of the plasma with by the incoming laser and showed few signs of filamentation. These differences are due to the longer distance over which the laser has to interact with low density plasma in the low-contrast case. This allows a longer distance for the non-linear effects to initiate and grow. The consequence of these non-linear effects is to accelerate electrons that are more divergence, which will decrease the fraction of electrons entering the cone-tip.

Thus we have identified a reason for the decreased amount of coupling of energy into the wires at low-contrast. This is due to an increased distance of where the electrons are accelerated from the cone-tip. It may also be due to the increased amount of non-linear effects such as hole-boring and filmentation, which create a more divergence electron beam.

\subsubsection{Electron Spectra and Falloff Length at Low-Contrast}

The other major difference between the two laser systems was the decrease in falloff distance in the low-contrast data. This implies that there is a cooler 
electron distribution in the low-contrast case. This is a puzzle, as other work has shown that increased levels of preplasma should increase the electron slope temperature. ${ }^{[8]}$ To understand this, we now look at the electron spectrum from the low-contrast case.

To look at the spectrum near to their source, the electrons are counted going through a plane located at $40 \mu \mathrm{m}$ from the cone-tip (-50 $\mu \mathrm{m}$ in Figure 8.13). To count electrons, they are radially weighted and the backwards going electrons are subtracted, as done with the high-contrast simulations. The electron spectrum is also observed at the cone-tip. These spectra are shown plotted along with the high-contrast spectra as presented previously in Figure 8.7. There is about a factor of $2.5 x$ decrease in total electron energy at distances $40 \mu \mathrm{m}$ away from the conetip compared to electrons passing through the cone-tip. However, the shape of the spectral profiles is relatively unchanged for these two locations. This indicates that a similar amount of high and low energy electrons were lost in the LPI simulations as the electrons traveled through the cone.

$\square$ HC: $0.1 \mu \mathrm{m}$
- HC: $1.0 \mu \mathrm{m}$
- HC: $3.0 \mu \mathrm{m}$
$\square-$ LC: at cone-tip
$-\square$ LC: $40 \mu \mathrm{m}$ away

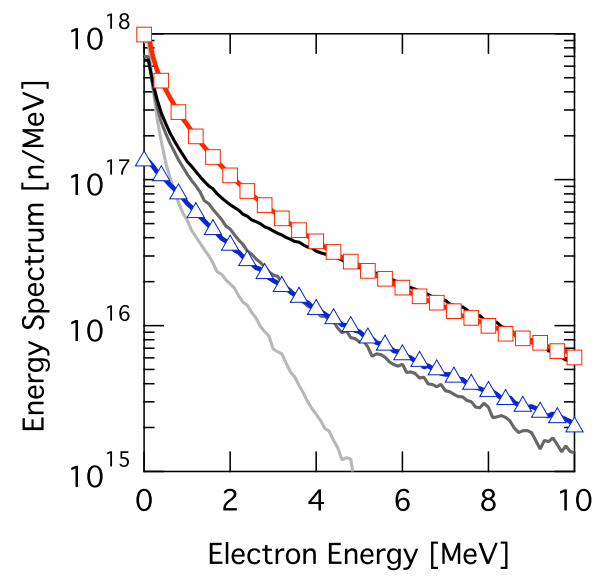

Figure 8.14: Energy spectrum of electrons in the low-contrast (LC) case compared to the high-contrast (HC) cases. All of the $\mathrm{HC}$ spectra are taken at the cone-tip, while the LC data is take both at the tip and at $40 \mu \mathrm{m}$ from the tip.

The spectra presented from the low-contrast data are shown to be hotter than the spectrum from the high-contrast simulations. This seems to contradict the experimental data, which implies that the electron distribution within the 
wire is cooler at low-contrast. This implies that there is another mechanism that is important in changing the falloff of $\mathrm{K}_{\alpha} \mathrm{x}$-ray emission.

We now investigate the possibility that the electron angular distribution plays a role in changing the $\mathrm{K}_{\alpha}$ emission profile. The angular profile of electrons of various energies are shown taken through the plane $40 \mu \mathrm{m}$ from the cone-tip in Figure 8.15. The lowest energy electrons, up to around $4 \mathrm{MeV}$, are relatively symmetric around the central axis and are not strongly filamented. These electrons show a roughly gaussian divergence that is wider than the other electrons. With higher energy electrons the story is different, the electrons become less symmetric and the divergence profile becomes more filamented. This shows how the laser profile filaments are imprinted upon the accelerated electrons. The hottest electrons, above $10 \mathrm{MeV}$ and to some extend, electrons above $4 \mathrm{MeV}$, are less divergent and more peaked in a given direction.
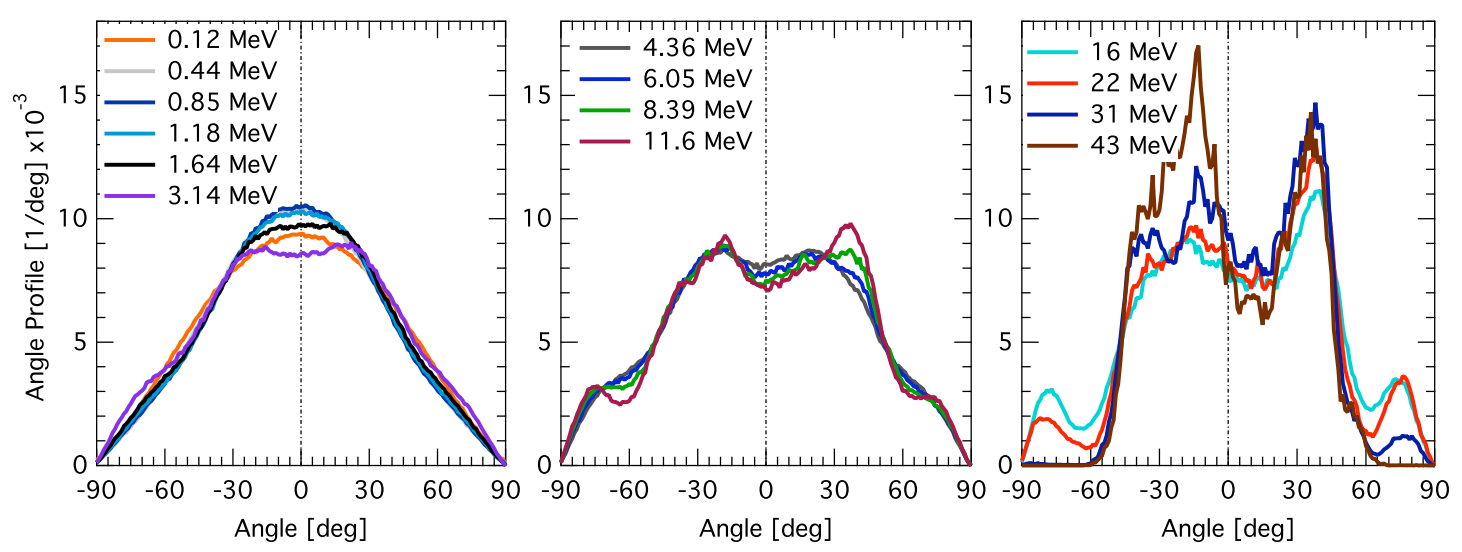

Figure 8.15: Angular divergence distributions of the electrons at different energies taken near the source at $40 \mu \mathrm{m}$ from the inner cone-tip.

The hotter electrons are not generally pointed straight forward and are instead angled around $30^{\circ}$ from the center. Therefore these electrons are not headed towards the cone-tip. These electrons should be more likely to leave the target before heading through the tip (i.e. through the side of the cone), especially as they are higher energy and thus more likely to overcome the fields on the cone walls. However, Figure 8.14 shows that the electron spectrum at the cone-tip is not dramatically different in slope, instead it is only lower in total number. This 
suggests that, in our simulations, the number of electrons lost between the source and the tip is independent of electron energy, despite the fact that many of the hotter electrons are not directed towards to cone-tip.

Since the LPI simulations require that the modeling is performed using 2D Cartesian geometry, the fields along the edges of the target will not be modeled correctly. This is because the target is naturally a cylindrical geometry. Thus the fields will be stronger in the LPI simulation that they would be in a cylindrical simulation. This increased field strength may cause even the hottest electrons to be confined within the cone. Thus the electron spectrum may be hotter in the LPI simulations than it would be if it was possible to perform 3D simulations.

To compare the results of the low-contrast simulations with the experimental data, the electron distributions are again injected into transport simulations using the same methods discussed previously in this chapter to inject electrons into the cone-tip. The results of this injection are shown in Figure 8.16. This shows that the falloff distance predicted from the LPI simulated distribution is too long to match the experimental data.

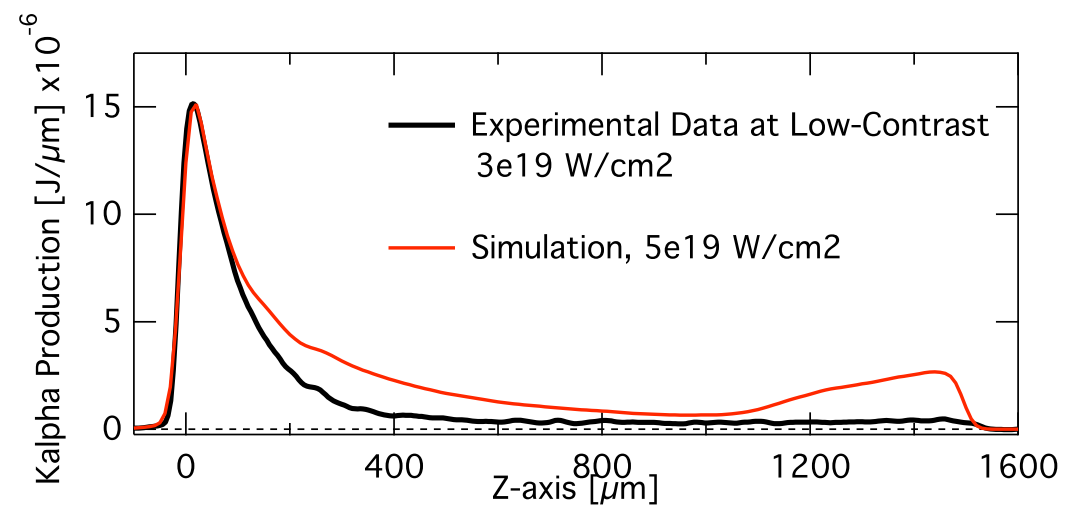

Figure 8.16: Profile of $\mathrm{K}_{\alpha}$ emission from the wire using the injected electron sources low-contrast simulations LPI simulation at $5 \times 10^{19} \mathrm{~W} / \mathrm{cm}^{2}$ and the experimental data from the $3 \times 10^{19} \mathrm{~W} / \mathrm{cm}^{2}$ case.

There are a few reasons why LPI simulations do not produce electrons that quantitatively match the experimental data. As explained in proceeding paragraphs the LPI simulations were modeled in 2D Cartesian which may have con- 
tained hotter electrons within the cone and lead to a hotter spectrum. Another major issue is the amount of self-focusing, filamentation and hole-boring observed in the low-contrast simulations. These processes are highly non-linear and in our case were modeled in 2D, such modeling neglects 3D effects which may be important in stabilizing or potentially magnifying such instabilities. Additionally, modeling the preplasma ablation is a difficult task, as it requires accurate knowledge of the absorption of laser light at different plasma and laser parameters, and an accurate description of the equation-of-state of the material. Both of which may be difficult to know with certainty, especially for high atomic number materials.

The difficulty in modeling the laser interaction with large amounts of preplasma once again highlights the advantage of using high-contrast lasers. Since, as was shown earlier in the chapter, the electrons are accelerated much closer to the tip and non-linear processes are much less prevalent.

Figure 8.17 plots the falloff distance in the wire against preplasma scale length. At lower levels of preplasma the falloff distance increases with increased preplasma. However, with the larger amount of preplasma in the Titan case, the falloff decreases. This can be attributed to the off-axis angles of the hottest electrons. As these most energetic electrons do not make it into the wire, the electron distribution within the wire is colder and thus has a decreased falloff distance.

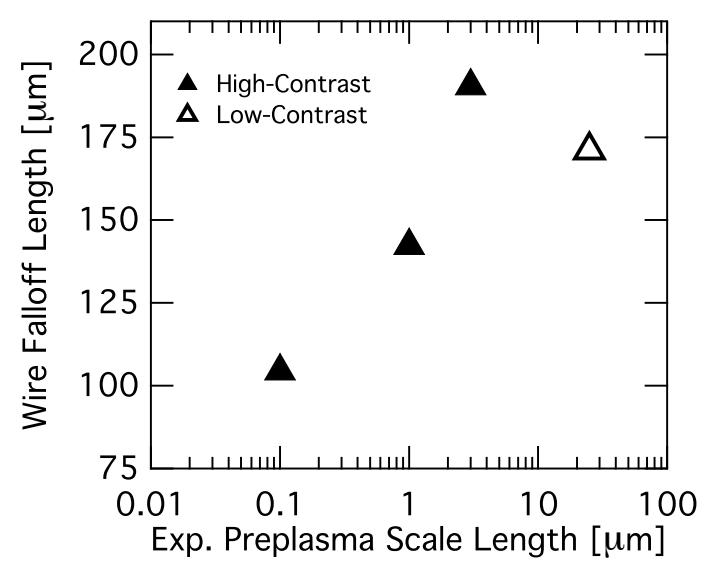

Figure 8.17: Simulated falloff distances as a function of preplasma scale length. 
Thus the trends of the falloff distance and preplasma are consistent between experiment and simulation, since larger values of preplasma show that the falloff distance begins to decrease. However, as we have explained, for a number of reasons, the low-contrast simulations are not able to quantitatively match the experimental data. As expressed previously, this is likely due to the restriction of using a 2D Cartesian geometry instead of the true 3D that will be present in experiment.

\subsection{LPI Discussion}

Simulations were performed to model the high-contrast Trident laser interaction with different lengths $(0.1$ to $3 \mu \mathrm{m})$ of initial preplasma. These simulations show that the electron spectrum gets hotter and the absorption of laser light increases with increased initial preplasma scale length. Also, this increase is coupled to broadening of each of the temporal, spatial and angular distributions. These changes decrease the fraction of the absorbed electrons that reach the inner tip of the cone, though the total number electrons reaching the tip increases. Knowledge of the complete electron distribution is used to inject electrons into transport simulations to allow comparison to experimental data. These simulations show that a 1.8 to $3 \mu \mathrm{m}$ preplasma scale length agrees with the experimental data. This amount of preplasma is shown to be physically reasonable based on a simple energy deposition model using the rising-time of the laser.

Additionally, simulations of longer scale preplasma were run to represent the low-contrast Titan case. This simulation showed a significantly different interaction of the laser with the target from the high-contrast case. Non-linear processes such as filamentation and hole-boring played a major role in the interaction and such processes were imprinted on the accelerated electrons. This interaction showed a major decrease in the coupling of electrons into the cone-tip, which was previously seen in the experimental data. On the other hand, the LPI simulations of lowcontrast did not give a quantitative fit to the experimentally observed $\mathrm{K}_{\alpha}$ falloffs. This is most likely due to the complicated nature of the long scale preplasma 
interaction, which is not modeled properly in a $2 \mathrm{D}$ geometry.

To our knowledge, this integration of both LPI and transport simulations is the most complete study of accelerated electron distributions using cone-wire targets to date. Using LPI to model all of the electron quantities means that this work does not rely on the ad-hoc assumptions our previous work in respect to the spatial, temporal and angular distributions of electrons. The removal of such assumptions gives more confidence that the spectra and distributions inferred in this study are more accurate representations of the accelerated electrons observed in experiment. 


\section{Summary}

This dissertation has studied the acceleration of electrons by ultra-highcontrast lasers using both experimental methods, analytical calculations and largescale simulations. This work is of particular interest to the fast ignition (FI) concept of inertial confinement fusion, where these electrons deposit energy within an imploded core to ignite fusion reactions. Previous work ${ }^{[38-41]}$ has shown that large amounts of preplasma (i.e. material ablated prior to the main pulse interaction) can dramatically reduce the coupling of these electrons into targets with similar geometries as the imploded core. For the first time, this dissertation investigated the acceleration electrons using a FI-relevant cone-wire geometry, where this preplasma was significantly reduced. This was done experimentally using an ultra-high-contrast laser, where the laser intensity coming nanoseconds prior to the main pulse, which is normally the cause of preplasma, has been eliminated. These experiments were interpreted using a combination of simulations to model both acceleration of electrons by the laser and the subsequent transport of these electrons through the target. These models allowed the full distribution (e.g. in space, time, angle, energy) of the electrons to be inferred and compared quantitatively to experiment. Such comprehensive modeling of these cone-wire target interactions has never before been realized.

The cone-wire targets used in this work were chosen with a similar geometry to the cone-guided FI concept. In the experiments, a hollow Au cone with a $10 \mu \mathrm{m}$ thick tip was aligned so that the laser traveled down the middle and was incident on the $30 \mu \mathrm{m}$ diameter inner tip. The outer tip of the cone was attached to a $40 \mu \mathrm{m}$ diameter $\mathrm{Cu}$ wire, which allowed electrons to be observed through theie emission of $\mathrm{K}_{\alpha} \mathrm{x}$-ray emission. The targets were irradiated at intensities from $2 \times 10^{19}$ 
to $5 \times 10^{19} \mathrm{~W} / \mathrm{cm}^{2}$ by the ultra-high-contrast Trident laser at the Los Alamos National Laboratory. Measurement of the total amount of $\mathrm{K}_{\alpha}$ x-ray generation from the wire allowed the coupling of electron energy into the wire to be assessed; this was found to be independent of the laser intensity. Imaging the falloff of $\mathrm{K}_{\alpha} \mathrm{X}-$ ray emission was used to infer an effective path length of electrons within the wire to diagnose their energy spectrum. A strong dependent on the laser intensity was found, indicating that electrons increase in energy with increased laser intensity.

To compare the ultra-high-contrast Trident case to conventional lasers, identical targets were irradiated by the low-contrast Titan laser at the Lawrence Livermore National Laboratory at intensities from $10^{19}$ to $10^{20} \mathrm{~W} / \mathrm{cm}^{2}$. Unlike the Trident laser, the Titan laser has amplified spontaneous emission (ASE) prepulse. The ASE prepulse irradiates the target with 1-17 mJ of energy in a time around $2 \mathrm{~ns}$ prior to the main pulse of the laser, which contained 16-160 J of energy in $0.7 \mathrm{ps}$. This amount of ASE is a hundred million $\left(10^{-8}\right)$ times less intense than the main pulse, yet it can ablate material extending hundreds of microns from the initial target surface. Our previous experiments using cone-wire targets ${ }^{[41]}$ showed a 100x increase in the prepulse energy reduced electron coupling into the wire by a factor of $10 \mathrm{x}$ due to the increased distance of the critical surface from the initial target surface. Instead of increased prepulse energy, this dissertation explores the coupling of electrons into a wire when the ASE prepulse is effectively eliminated using high-contrast, which until now has not been quantitatively assessed. Measurement of the total $\mathrm{K}_{\alpha}$ x-ray emission from the wire indicated that $2.7 \mathrm{x}$ more electrons are coupled into the wire at high-contrast, indicating that reduction of ASE prepulse is worthwhile investment for laser systems relevant to FI research.

To quantitatively understand the role of preplasma on electron generation simulations of the laser-plasma-interaction (LPI) were performed using the particle-in-cell (PIC) code LSP. ${ }^{[55]}$ These simulations investigated exponential preplasma scale lengths from 0.1 to $3 \mu \mathrm{m}$, as well as the longer preplasma expected from the low-contrast Titan laser. These simulations showed that the absorption of laser light increases dramatically with preplasma from around $20 \%$ at low, 0.1 um, scale lengths to nearly $90 \%$ at low-contrast-type preplasma. However, this 
increase in absorbed energy is not all given to electrons that will reach the wire. Instead the electrons are broadened in space in space, which is caused by the longer distance over which electrons have to diverge and their increased divergence angle with preplasma. This leads to a reduction in the amount of energy that reaches the cone-tip at long preplasma scale lengths and is the cause for the $2.7 \mathrm{x}$ decrease in coupling with low-contrast-type preplasma observed.

The electron energy spectrum generated from the LPI simulations was characterized by a three-temperature exponential distribution function. The hottest part of the spectrum was characterized by a temperature on the order of the socalled ponderomotive energy $\left(2.78 \mathrm{MeV}\right.$ at $\left.5 \times 10^{19} \mathrm{~W} / \mathrm{cm}^{2}, \lambda_{L}=1.054 \mu \mathrm{m}\right)$, while the lowest temperature was on the order of a few hundred $\mathrm{keV}$. Increasing the preplasma scale length from 0.1 to $3 \mu \mathrm{m}$ caused the slope temperature of these fits to increase by about a factor of $2.5 \mathrm{x}$. The total number of accelerated electrons was roughly the same in these simulations. This indicates that the increase in absorbed energy with longer preplasma levels is caused by individual electrons being accelerated to higher energies rather than an increase in the total number of electrons being accelerated. This is consistent with a longer length over which electrons can gain energy from the laser.

The electron distributions determined from LPI simulations were next compared to the experimental data. This was done by modeling the electron transport through the cone-wire target using, once again, the PIC code LSP. Unlike the LPI simulations, which focused on the region of laser interaction, these simulations included the full-scale geometry of the target and included large boundaries, so that electric and magnetic fields developing along the target were fully modeled. The electrons characterized in the LPI simulations were injected at the cone-tip and traveled through the target to produce $\mathrm{K}_{\alpha} \mathrm{x}$-ray emission that was compared with the experimental data. These simulations were able to reproduce all of the important features of the $\mathrm{K}_{\alpha} \mathrm{x}$-ray emission profile.

One interesting feature in the experimental data is a bump of emission at the end of the wire. Using the transport simulations this bump was shown to be caused by electrons that surf along the edge of the until they reach the end, 
at which point they are pushed into the wire and create the bump of emission. These surfing electrons are held along the wire by strong radial electric fields that create electric potentials on the order of MV. In opposition to this, an azimuthal magnetic field on the order of MG, creates a force which pushes electrons away from the wire. This magnetic field is caused by electrons traveling in opposition to the forward traveling electrons. Once electrons reach the end of the wire this opposing current can no longer propagate. Thus there is no magnetic field to push the surfing electrons away from the wire and they are pushed into the wire by the radial electric field, thus creating a bump of $\mathrm{K}_{\alpha}$ emission.

In terms of understanding the electron spectrum, the most important feature of the $\mathrm{K}_{\alpha}$ emission profile is the initial falloff; essentially, the effective path length of electrons in the wire. The transport simulations, and supporting analytical calculations, show that the slope of this falloff is sensitive to electrons with kinetic energies around 0.5 to $2.5 \mathrm{MeV}$. Thus matching this slope gives confidence in the validity of the LPI simulated electrons. The electron spectrum that best matches the falloff of the $5 \times 10^{19} \mathrm{~W} / \mathrm{cm}^{2}$ experimental data at high-contrast is found with an initial $3 \mu \mathrm{m}$ preplasma scale length. This implies that such a scale length was present even with the ultra-high-contrast Trident laser.

A $3 \mu \mathrm{m}$ preplasma scale length is somewhat unexpected, as previous simulations ${ }^{[18]}$ had supposed that only a minimal $(0.5 \mu \mathrm{m})$ scale length was present for such high-contrast interactions due to the lack of ASE prepulse. However, the Trident laser does have a finite rising-time, which occurs less than 0.1 ns prior to the main pulse. The effect of this rising-time is investigated using a simple analytical calculation: Using the experimental measurement of the rising-time of the laser ${ }^{[18]}$ the target is allowed to absorb energy with a given absorption efficiency. ${ }^{[92]}$ The increase in energy causes the target to increase in temperature and ionization state through an equation-of-state, ${ }^{[85,86]}$ which then cause the target to expand. Such calculations verify that many microns of preplasma can indeed be ablated from the $0.1 \mathrm{~ns}$ rising-time of the Trident laser.

In summary, this dissertation has coupled LPI and transport simulations to understand laser phenomena using ultra-high-contrast lasers and made quan- 
titative comparisons with experimental data. Such an integrated approach is a recent development in FI research and the ability to get quantitative agreement between simulations and experiment is a testament to the large amount of previous research that has gone into developing these codes to include many important pieces of physics.

This work shows promise for Fast Ignition research in that through reduction of the ASE prepulse to negligible levels the coupling of electrons into the wire is increased by nearly a factor of $3 \mathrm{x}$. Such information shows upgrading laser facilities to incorporate ultra-high-contrast is a worthwhile investment. The knowledge that even the rising-edge of the laser may ablate a relatively large amount of preplasma will be quite useful ultra-high-contrast facilities become more wide spread. Additionally, the detailed investigation of low $(0.1$ to $3 \mu \mathrm{m})$ preplasma scale length will aid researchers in understanding how the electron energy spectrum, as well as the spatial, temporal and angular distributions, can change with preplasma. 


\section{A Transmission of a 3D cylinder}

We begin by setting up a cylinder along the z-axis and we place an observer at infinity so that this person is always looking perpendicular to the $\mathrm{y}$-axis, this is justified if the distance to observer $>>R$. There is no dependence on $z$ so we will treat the problem as a circle for now (see Figure A.2) and do the z-axis later.

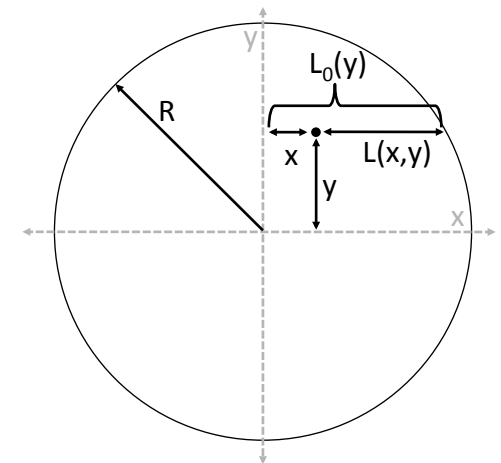

Figure A.1: Sketch of a circle being viewed by observer at $x=\infty$. $L$ is the distance that the observer must view to see the point at $(\mathrm{x}, \mathrm{y})$ and $L_{0}$ is the length parallel to the $\mathrm{x}$-axis from the outer edge of the cricle to the y-axis.

The emission of the circle is $d E=\rho e^{-L / \lambda} d x d y d z$, where $\rho$ is the emission density, $L$ is the length to the observer and $\lambda$ is the attenuation length. Which gives following equation (A.3). Next we notice that the $L=L_{0}-x$ and through the pythagorean theorem $L_{0}=\sqrt{R^{2}-y^{2}}$.

$$
\begin{aligned}
E(R, \lambda, \rho) & =\rho \int_{-R}^{R} \int_{-L_{0}}^{L_{0}} \int_{0}^{H} \exp \left(-\frac{L}{\lambda}\right) d z d x d y \\
& =\rho \int_{-R}^{R} \int_{-L_{0}}^{L_{0}} \int_{0}^{H} \exp \left(-\frac{L_{0}-x}{\lambda}\right) d z d x d y
\end{aligned}
$$


The integral over $z$ is simple and $x$ is not so difficult.

$$
\begin{aligned}
E(R, \lambda, \rho) & =H \rho \lambda \int_{-R}^{R}\left[1-\exp \left(\frac{2 L_{0}}{\lambda}\right)\right] d y \\
& =2 H \rho \lambda-H \rho \lambda \int_{-R}^{R} \exp \left(\frac{2 \sqrt{R^{2}-y^{2}}}{\lambda}\right) d y
\end{aligned}
$$

We will define the rightmost side of the equation as $\psi(R, \lambda)$ for simplicity (eqn(A.5)). Then we do a substitution into $\theta$ by making a triangle so that $R \cos \theta=\sqrt{R^{2}-y^{2}}$ and $d y=R \cos \theta d \theta$.

$$
\begin{aligned}
\psi(R, \lambda) & \equiv \int_{-R}^{R} \exp \left(-\frac{2}{\lambda} \sqrt{R^{2}-y^{2}}\right) d y \\
& =\int_{-\pi / 2}^{\pi / 2} \exp \left(-\frac{2}{\lambda} R \cos \theta\right) R \cos \theta d \theta
\end{aligned}
$$

This has a solution that can be found at Wolfram|Alpha ${ }^{[93]}$ and it is:

$$
\psi(R, \lambda)=\pi R\left[L_{-1}\left(\frac{2 R}{\lambda}\right)-I_{1}\left(\frac{2 R}{\lambda}\right)\right]
$$

Where $I_{n}(z)$ is the is the modified Bessel function of the first kind and $L_{n}(x)$ is the modified Struve function. Now we can show that the final solution of $E$ is:

$$
E(R, \lambda, \rho)=2 H \rho \lambda R-H \rho \lambda \pi R\left[L_{-1}\left(\frac{2 R}{\lambda}\right)-I_{1}\left(\frac{2 R}{\lambda}\right)\right]
$$

Now for an observer looking at the view with a given view angle $\gamma$, the length that is seen is In order to include any view angles of the observer of the wire, we can see that the actual length seen is $L_{V}=L / \cos \gamma$. To make this easier on ourselves we can say that $e^{-L_{V} / \lambda}=e^{-L / \lambda_{\gamma}}$, where $\lambda_{\gamma}=\lambda \cos \gamma$.

Substituting in $\lambda_{\gamma}$ for $\lambda$ we get the final answer. Note that we are neglecting the edges of end edge of the wire, where $z \approx H$ for $\gamma>0$ or $z \approx H$ for $\gamma<0$. However, we are more interested in the transmission, $T$, of the wire so we will set $\rho=\left(\pi R^{2} H\right)^{-1}$. Also, shown as a function of $Z \equiv \frac{R}{\lambda \cos \gamma}$.

$$
T(R, \lambda, \gamma)=\frac{\lambda \cos \gamma}{R}\left[\frac{2}{\pi}-L_{-1}\left(\frac{2 R}{\lambda \cos \gamma}\right)+I_{1}\left(\frac{2 R}{\lambda \cos \gamma}\right)\right]
$$




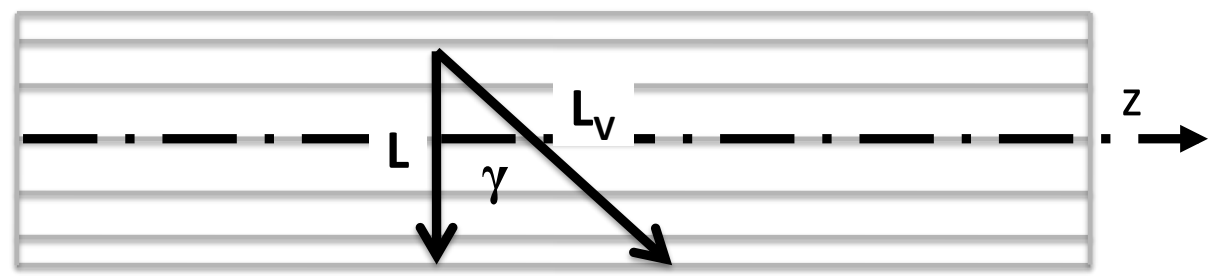

Figure A.2: Sketch of a cylinder where $\gamma$ is the view angle, $L$ is the length to observer without the view angle and $L_{V}$ is the actually length to observer including the view angle.

$$
T(Z)=\frac{1}{Z}\left[\frac{2}{\pi}-L_{-1}(2 Z)+I_{1}(2 Z)\right]
$$

For a wire with $R=20 \mu \mathrm{m}, \lambda=22.21(\mathrm{Cu}$ at $8037.8 \mathrm{eV}), \rho=\pi R^{2}$ and $\gamma=45^{\circ}$, the transmission is $T=0.415$ and the opacity, $\frac{1}{T}$ is 2.41 .

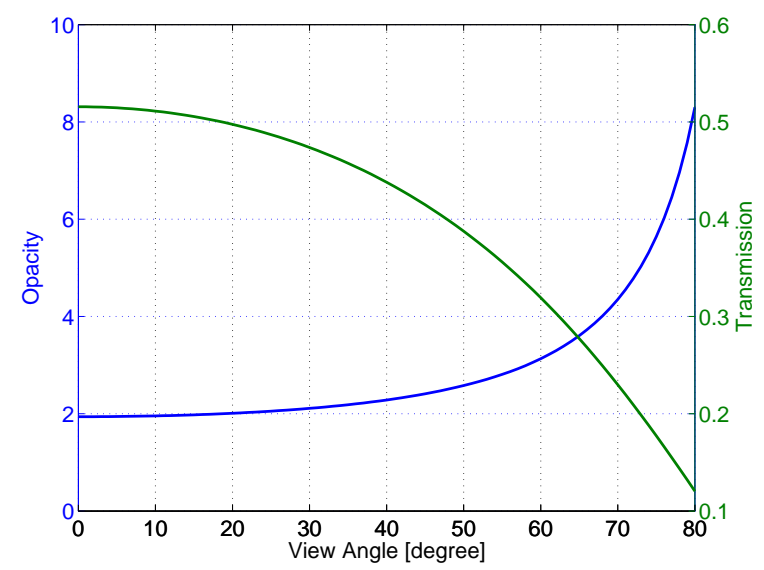

Figure A.3: The transmission, $T(R=20 \mu \mathrm{m}, \lambda=22 \mu \mathrm{m}, \gamma)$, and opacity $(\equiv 1 / T)$ plotted against the view angle of the wire, $\gamma$.

The wires used in most of our experiments were $20 \mu \mathrm{m}$ radius, $\mathrm{Cu}$ wires. $\mathrm{Cu}$ emits $\mathrm{K} \alpha$ photons at $8 \mathrm{keV}$, the attenuation length of $\mathrm{Cu}$ at this energy is 22 $\mu \mathrm{m}$. Using the equation above transmission, $T(R, \lambda, \gamma)$, for a given view angle can be found. This is shown in Figure A.3 and Table A.1. 
Table A.1: Transmission, $T(R=20 \mu \mathrm{m}, \lambda=22 \mu \mathrm{m}, \gamma)$, and opacity $(\equiv 1 / T)$ for view angles, $\gamma$.

\begin{tabular}{ccc}
\hline View Angle & Transmission & Opacity \\
\hline $0^{\circ}$ & 0.52 & 1.94 \\
$5^{\circ}$ & 0.52 & 1.94 \\
$10^{\circ}$ & 0.51 & 1.96 \\
$15^{\circ}$ & 0.51 & 1.98 \\
$20^{\circ}$ & 0.50 & 2.01 \\
$25^{\circ}$ & 0.49 & 2.05 \\
$30^{\circ}$ & 0.47 & 2.11 \\
$35^{\circ}$ & 0.46 & 2.19 \\
$40^{\circ}$ & 0.44 & 2.28 \\
$45^{\circ}$ & 0.41 & 2.41 \\
$50^{\circ}$ & 0.39 & 2.58 \\
$55^{\circ}$ & 0.36 & 2.81 \\
$60^{\circ}$ & 0.32 & 3.13 \\
$65^{\circ}$ & 0.28 & 3.61 \\
$70^{\circ}$ & 0.23 & 4.35 \\
$75^{\circ}$ & 0.18 & 5.64 \\
$80^{\circ}$ & 0.12 & 8.3
\end{tabular}




\section{Bibliography}

[1] D. Strickland and G. Mourou, Optics Communications 55, 447-449 (1985).

[2] X. Wang, D. Du, S. Yi, S. Kalmykov, E. D'avignon, N. Fazel, R. Zagdzaj, S. Reed, P. Dong, and W. Henderson, ADVANCED ACCELERATOR CONCEPTS: 14th Advanced Accelerator Concepts Workshop. AIP Conference Proceedings 1299, 209 (2010).

[3] V. Yanovsky, V. Chvykov, G. Kalinchenko, P. Rousseau, T. Planchon, T. Matsuoka, A. Maksimchuk, J. Nees, G. Cheriaux, and G. Mourou, Optics Express 16, 2109-2114 (2008).

[4] Laboratory for Laser Energetics, "LLE 2011 Annual Report," tech. rep., University of Rochester, (2011).

[5] International Energy Agency, "Key world energy statistics," (2010).

[6] C. Chen, P. Patel, D. Hey, A. Mackinnon, M. Key, K. Akli, T. Bartal, F. Beg, S. Chawla, and H. Chen, Physics of Plasmas 16, 082705 (2009).

[7] F. Perez, L. Gremillet, M. Koenig, S. Baton, P. Audebert, M. Chahid, C. Rousseaux, M. Drouin, E. Lefebvre, and T. Vinci, Physical review letters 104, 85001 (2010).

[8] K. Akli, S. Hansen, A. Kemp, R. Freeman, F. Beg, D. Clark, S. Chen, D. Hey, S. Hatchett, K. Highbarger, E. Giraldez, J. Green, G. Gregori, K. Lancaster, T. Ma, A. Mackinnon, P. Norreys, N. Patel, J. Pasley, C. Shearer, R. Stephens, C. Stoeckl, M. Storm, W. Theobald, L. V. Woerkom, R. Weber, and M. Key, Physical review letters 100, 165002 (2008).

[9] R. P. Drake, High-Energy-Density Physics: Fundamentals, Inertial Fusion, and Experimental Astrophysics. Springer, (2006).

[10] A. Fews, P. Norreys, F. Beg, A. Bell, A. Dangor, C. Danson, P. Lee, and S. Rose, Physical review letters 73, 1801-1804 (1994).

[11] R. Snavely, M. Key, S. Hatchett, T. Cowan, M. Roth, T. Phillips, M. Stoyer, E. Henry, T. Sangster, and M. Singh, Physical review letters 85, 2945-2948 (2000). 
[12] S. Wilks, A. Langdon, T. Cowan, M. Roth, M. Singh, S. Hatchett, M. Key, D. Pennington, A. Mackinnon, and R. Snavely, Physics of Plasmas 8, 542 (2001).

[13] M. Hegelich, S. Karsch, G. Pretzler, D. Habs, K. Witte, W. Guenther, M. Allen, A. Blazevic, J. Fuchs, and J. Gauthier, Physical review letters 89, 85002 (2002).

[14] A. Mackinnon, Y. Sentoku, P. Patel, and D. Price, Physical review letters (2002).

[15] P. Patel, A. Mackinnon, M. Key, T. Cowan, M. Foord, M. Allen, D. Price, H. Ruhl, P. Springer, and R. Stephens, Physical review letters 91, 125004125004 (2003).

[16] J. Fuchs, P. Antici, E. D-[Rsquo]-Humi-[Egrave]-Res, E. Lefebvre, M. Borghesi, E. Brambrink, C. A. Cecchetti, M. Kaluza, V. Malka, M. Manclossi, S. Meyroneinc, P. Mora, J. Schreiber, T. Toncian, H. P-[Eacute]-Pin, and P. Audebert, Nature Physics 2, 48 (2005).

[17] L. Robson, P. T. Simpson, R. J. Clarke, K. W. D. Ledingham, F. Lindau, O. Lundh, T. Mccanny, P. Mora, D. Neely, C. G. Wahlstr-[Ouml]-M, M. Zepf, and P. Mckenna, Nature Physics 3, 58 (2006).

[18] S. A. Gaillard, T. Kluge, K. A. Flippo, M. Bussmann, B. Gall, T. Lockard, M. Geissel, D. T. Offermann, M. Schollmeier, Y. Sentoku, and T. E. Cowan, Physics of Plasmas 18, 056710 (2011).

[19] T. Bartal, M. E. Foord, C. Bellei, M. H. Key, K. A. Flippo, S. A. Gaillard, D. T. Offermann, P. K. Patel, L. C. Jarrott, D. P. Higginson, M. Roth, A. Otten, D. Kraus, R. B. Stephens, H. S. McLean, E. M. Giraldez, M. S. Wei, D. C. Gautier, and F. N. Beg, Nature Physics 7, 1-4 (2011).

[20] K. L. Lancaster, S. Karsch, H. Habara, F. N. Beg, E. L. Clark, R. Freeman, M. H. Key, J. A. King, R. Kodama, K. Krushelnick, K. W. D. Ledingham, P. Mckenna, C. D. Murphy, P. A. Norreys, R. Stephens, C. Stoeckl, Y. Toyama, M. S. Wei, and M. Zepf, Physics of Plasmas 11, 3404 (2004).

[21] D. P. Higginson, J. McNaney, D. Swift, T. Bartal, D. Hey, R. Kodama, S. L. Pape, A. Mackinnon, D. Mariscal, H. Nakamura, N. Nakanii, K. Tanaka, and F. Beg, Physics of Plasmas 17, 100701 (2010).

[22] D. P. Higginson, J. M. Mcnaney, D. C. Swift, G. M. Petrov, J. Davis, J. A. Frenje, L. C. Jarrott, R. Kodama, K. L. Lancaster, A. J. Mackinnon, H. Nakamura, P. K. Patel, G. Tynan, and F. N. Beg, Physics of Plasmas 18, 100703 (2011).

[23] H. Alfvén, Phys. Rev. 55, 425 (1939).

[24] A. Bell, J. Davies, S. Guerin, and H. Ruhl, Plasma Phys. Control. Fusion 39, 653 (1997). 
[25] M. Tabak, J. Hammer, M. Glinsky, W. Kruer, S. Wilks, J. Woodworth, E. Campbell, M. Perry, and R. Mason, Phys. Plasmas 1, 1626-1634 (1994).

[26] L. Meitner and O. Frisch, Nature 143, 239-240 (1939).

[27] O. Hahn and F. Strassman, Naturwiss 27, 11 (1939).

[28] E. Fermi, Proceedings of the American Philosophical Society 90, 20-24 (1946).

[29] J. Hakim, The Story of Science: Einstein Adds a New Dimension. Smithsonian Books, (2007).

[30] Atzeni and Meyer-Ter-Vehn, The Physics of Inertial Fusion. Oxford Univesity Press, (2004).

[31] H. Bosch and G. Hale, Nuclear fusion 32, 611 (1992).

[32] J. Lawson, Proceedings of the Physical Society. Section B 70, 6 (1957).

[33] S. Atzeni, A. Schiavi, and J. Davies, Plasma Phys. Control. Fusion 51, 015016 (2009).

[34] P. Norreys, R. Allott, R. Clarke, J. Collier, D. Neely, S. Rose, M. Zepf, M. Santala, A. Bell, and K. Krushelnick, Physics of Plasmas 7, 3721 (2000).

[35] R. Kodama, P. A. Norreys, K. Mima, A. E. Dangor, R. G. Evans, H. Fujita, Y. Kitagawa, K. Krushelnick, T. Miyakoshi, N. Miyanaga, T. Norimatsu, S. J. Rose, T. Shozaki, K. Shigemori, A. Sunahara, M. Tampo, K. A. Tanaka, Y. Toyama, T. Yamanaka, and M. Zepf, Nature 412, 798 (2001).

[36] R. Kodama, H. Shiraga, K. Shigemori, Y. Toyama, S. Fujioka, H. Azechi, H. Fujita, H. Habara, T. Hall, Y. Izawa, T. Jitsuno, Y. Kitagawa, K. M. Krushelnick, K. L. Lancaster, K. Mima, K. Nagai, M. Nakai, H. Nishimura, T. Norimatsu, P. A. Norreys, S. Sakabe, K. A. Tanaka, A. Youssef, M. Zepf, and T. Yamanaka, Nature 418, 933-934 (2002).

[37] H. D. Shay, P. Amendt, D. Clark, D. Ho, M. Key, J. Koning, M. Marinak, D. Strozzi, and M. Tabak, Physics of Plasmas (2012).

[38] L. V. Woerkom, K. U. Akli, T. Bartal, F. N. Beg, S. Chawla, C. D. Chen, E. Chowdhury, R. R. Freeman, D. Hey, M. H. Key, J. A. King, A. Link, T. Ma, A. J. Mackinnon, A. G. Macphee, D. Offermann, V. Ovchinnikov, P. K. Patel, D. W. Schumacher, R. B. Stephens, and Y. Y. Tsui, Physics of Plasmas 15, 056304 (2008).

[39] S. D. Baton, M. Koenig, J. Fuchs, A. Benuzzi-Mounaix, P. Guillou, B. Loupias, T. Vinci, L. Gremillet, C. Rousseaux, M. Drouin, E. Lefebvre, F. Dorchies, C. Fourment, J. J. Santos, D. Batani, A. Morace, R. Redaelli, M. Nakatsutsumi, R. Kodama, A. Nishida, N. Ozaki, T. Norimatsu, Y. Aglitskiy, S. Atzeni, and A. Schiavi, Physics of Plasmas 15, 042706 (2008).

[40] A. MacPhee, L. Divol, A. Kemp, K. Akli, F. Beg, C. Chen, H. Chen, D. Hey, R. Fedosejevs, and R. Freeman, Physical review letters 104, 55002 (2010). 
[41] T. Ma, H. Sawada, P. Patel, C. Chen, L. Divol, D. Higginson, A. Kemp, M. Key, D. Larson, S. L. Pape, A. Link, A. Macphee, H. Mclean, Y. Ping, R. Stephens, S. Wilks, and F. Beg, Physical review letters 108, 115004 (2012).

[42] A. J. Kemp, B. I. Cohen, and L. Divol, Physics of Plasmas 17, 056702 (2010).

[43] H. Sawada, D. P. Higginson, A. Link, T. Ma, S. C. Wilks, H. S. McLean, F. Perez, P. K. Patel, and F. N. Beg, Physics of Plasmas (2012).

[44] S. Wilks, W. Kruer, M. Tabak, and A. Langdon, Physical review letters 69, 1383-1386 (1992).

[45] F. N. Beg, A. R. Bell, A. E. Dangor, C. N. Danson, A. P. Fews, M. E. Glinsky, B. A. Hammel, P. Lee, P. A. Norreys, and M. Tatarakis, Physics of Plasmas 4, 447 (1997).

[46] A. Link, R. R. Freeman, D. W. Schumacher, and L. D. V. Woerkom, Physics of Plasmas (2011).

[47] B. Westover, C. D. Chen, P. K. Patel, M. H. Key, H. Mclean, R. Stephens, and F. N. Beg, Physics of Plasmas 18, 063101 (2011).

[48] M. J. Berger, J. S. Coursey, M. A. Zucker, and J. Chang, "ESTAR, PSTAR, and ASTAR: Computer Programs for Calculating Stopping-Power and Range Tables for Electrons, Protons, and Helium Ions (version 1.2.3)."

[49] R. Kodama, Y. Sentoku, Z. Chen, G. Kumar, S. Hatchett, Y. Toyama, T. Cowan, R. Freeman, J. Fuchs, and Y. Izawa, Nature 432, 1005 (2004).

[50] J. Green, K. Lancaster, K. Akli, C. Gregory, F. Beg, S. Chen, D. Clark, R. Freeman, S. Hawkes, and C. Hernandez-Gomez, Nature Physics 3, 853856 (2007).

[51] J. A. King, K. U. Akli, R. R. Freeman, J. Green, S. P. Hatchett, D. Hey, P. Jamangi, M. H. Key, J. Koch, K. L. Lancaster, T. Ma, A. J. Mackinnon, A. Macphee, P. A. Norreys, P. K. Patel, T. Phillips, R. B. Stephens, W. Theobald, R. P. J. Town, L. V. Woerkom, B. Zhang, and F. N. Beg, Physics of Plasmas 16, 020701 (2009).

[52] T. Ma, M. Key, R. Mason, K. Akli, R. Daskalova, R. Freeman, J. Green, K. Highbarger, P. Jaanimagi, and J. King, Physics of Plasmas 16, 112702 (2009).

[53] K. Akli, M. Key, H. Chung, S. Hansen, R. Freeman, M. Chen, G. Gregori, S. Hatchett, D. Hey, and N. Izumi, Physics of Plasmas 14, 023102 (2007).

[54] T. Ma, Electron Generation and Transport in Intense Relativistic LaserPlasma Interactions Relevant to Fast Ignition ICF. PhD thesis, University of California-San Diego, (2010).

[55] D. Welch, D. Rose, M. Cuneo, R. Campbell, and T. Mehlhorn, Physics of Plasmas 13, 063105 (2006). 
[56] D. P. Higginson, Bulletin of the American Physical Society 56 (2011).

[57] P. Gibbon, Short Pulse Laser Interactions with Matter. Imperial College Press, (2007).

[58] T. Carlson, C. Nestor, N. Wasserman, and J. McDowell, Atomic Data and Nuclear Data Tables 2, 63-99 (1970).

[59] M. V. Ammosov, N. Delone, and V. P. Kraino, JETP 1-4 (1986).

[60] A. V. Gurevich, L. V. Pariiskaya, and L. P. Pitaevskii, Zh. Eksp. Teor. Fiz. 49, 647 (1965). [Sov. Phys. JETP 22, 449 (1966)].

[61] J. E. Crow, P. L. Auer, and J. E. Allen, Journal of Plasma Physics 14, 65-76 (1975).

[62] W. Kruer, The Physics of Laser Plasma Interactions. Westview Press, (2003).

[63] L. Spitzer Jr., Physics of Fully Ionized Gases. Dover Books on Physics, second ed., (2006).

[64] Y. T. Lee and R. M. More, Physics of Fluids 27, 1273 (1984).

[65] M. Desjarlais, Contrib. Plasma Phys. 41, 267-270 (2001).

[66] J. Davies, Phys. Rev. E 69, 065402 (2004).

[67] J. R. Davies, Laser and Particle Beams 24, 299-310 (2006).

[68] A. Solodov and R. Betti, Physics of Plasmas 15, 042707 (2008).

[69] R. C. Shah, R. P. Johnson, T. Shimada, K. A. Flippo, J. C. Fernandez, and B. M. Hegelich, Opt Lett 34, 2273 (2009).

[70] E. Casnati, A. Tartari, and C. Baraldi, Journal of Physics B: Atomic and Molecular Physics 15, 155 (1982).

[71] C. Hombourger, Journal of Physics B: Atomic, Molecular and Optical Physics 31, 3693 (1998).

[72] B. R. Maddox, H. S. Park, B. A. Remington, and M. Mckernan, Rev. Sci. Instrum. 79, 10E924 (2008).

[73] N. Izumi, R. Snavely, G. Gregori, J. Koch, H. Park, and B. Remington, Rev. Sci. Instrum. 77, 10E325 (2006).

[74] C. K. Birdsall and A. B. Langdon, Plasma Physics Via Computer Simulation. CRC Press, (2005).

[75] M. Jones, D. Lemons, R. Mason, V. Thomas, and D. Winske, Journal of Computational Physics 123, 169-181 (1996).

[76] J. R. Davies, Phys. Rev. E 65, 1-14 (2002).

[77] D. J. Strozzi, M. Tabak, D. J. Larson, L. Divol, A. J. Kemp, C. Bellei, M. M. Marinak, and M. H. Key, Physics of Plasmas 19, 072711 (2012). 
[78] J. A. Halbleib, R. P. Kensek, G. D. Valdez, S. M. Seltzer, , and M. J. Berger, IEEE Trans. Nucl. Sci 39, 1025 (1992).

[79] D. Groom and S. Klein, The European Physical Journal C-Particles and Fields 15, 163-173 (2000).

[80] H. Kolbenstvedt, J. Appl. Phys. 38, 4785-4787 (1967).

[81] A. K. F. Haque, M. A. Uddin, M. A. R. Patoary, A. K. Basak, M. R. Talukder, B. C. Saha, K. R. Karim, and F. B. Malik, The European Physical Journal D-Atomic, Molecular, Optical and Plasma Physics 42, 203-210 (2007).

[82] M. Liu, Z. An, C. Tang, Z. Luo, X. Peng, and X. Long, Atomic Data and Nuclear Data Tables 76, 213-234 (2000).

[83] J. Myatt, W. Theobald, J. Delettrez, C. Stoeckl, M. Storm, T. Sangster, A. Maximov, and R. Short, Physics of Plasmas 14, 056301 (2007).

[84] W. Bambynek, B. Crasemann, R. Fink, H. Freund, H. Mark, C. Swift, R. Price, and P. Rao, Reviews of Modern Physics 44, 716-813 (1972).

[85] J. J. MacFarlane, I. E. Golovkin, and P. R. Woodruff, J. Quant. Spec. Rad. Transf. 99, 381 (2006).

[86] J. MacFarlane, I. E. Golovkin, P. Wang, P. R. Woodruff, and N. A. Pereyra, High Energy Density Physics 3, 181 (2007).

[87] M. Allen, P. Patel, A. Mackinnon, D. Price, S. Wilks, and E. Morse, Physical review letters 93, 265004 (2004).

[88] A. J. Kemp, Y. Sentoku, and M. Tabak, Physical review letters 101 (2008).

[89] J. D. Jackson, Am. J. Phys 68, 789-799 (2000).

[90] F. Jüttner, Annalen der Physik 340, 145-161 (1911).

[91] J. Dunkel and P. Hänggi, Physics Reports 471, 1-73 (2009).

[92] P. Mora, Physics of Fluids 25, 1051 (1982).

[93] Wolfram|Alpha, www.wolframalpha.com (2012). 